\title{
HIGH RESOLUTION RADIO OBSERVATIONS OF ENERGETICALLY DOMINANT REGIONS IN LOCAL LUMINOUS INFRARED GALAXIES
}

\author{
Loreto de los Ángeles Barcos Muñoz \\ Santiago, Chile
}

B.S. in Applied Physics, Universidad de Santiago de Chile, 2007

M.S. in Astronomy, University of Virginia, 2012

A Dissertation Presented to the Graduate

Faculty of the University of Virginia

in Candidacy for the Degree of

Doctor of Philosophy

Department of Astronomy

University of Virginia

August, 2016

Committee Members:

Adam K. Leroy

Aaron S. Evans

Phil Arras

Robert Davis

Eric J. Murphy 
(C) Copyright by

Loreto de los Ángeles Barcos Muñoz

All rights reserved

July 26, 2016 


\begin{abstract}
Luminous and Ultra-luminous Infrared galaxies (U/LIRGs) are one of the most powerful classes of extragalactic objects in the local universe, and they provide a unique opportunity to study star formation and feedback processes in extreme environments. They are primarily observed to be interacting or merging disk galaxies. During the interaction, large amounts of gas are funneled to the central few kpc, triggering high star formation rates (SFR) and dust production. The absorption of UV and optical radiation from stars, or active galactic nuclei (AGN), by dust produces their observed high infrared luminosities.

The high level of dust obscuration intrinsic to U/LIRGs makes them difficult to study. Radio interferometry is thus the perfect tool for revealing the nature of these systems - it provides the high spatial resolution needed to resolve energetically dominant regions in U/LIRGs at wavelengths that have both diagnostic power and transparency to dust. In this thesis, 6 and $33 \mathrm{GHz}$ radio continuum interferometric observations with the upgraded Karl G. Jansky Very Large Array (VLA) are used to study a sample of 22 local U/LIRGs.

First, a detailed analysis of the 6 and $33 \mathrm{GHz}$ radio continuum emission from the closest ULIRG, Arp 220, is presented. This late stage merger is highly obscured, being optically thick even at mid-infrared wavelengths. Further, due to its extreme environment, it is often used as a template for high redshift starbursts. Arp 220 hosts two distinct nuclei that are separated by $\sim 370 \mathrm{pc}$. The nuclei are well resolved with the $33 \mathrm{GHz}$ observations (i.e., with a spatial resolution of $\sim 30 \mathrm{pc}$ ). The deconvolved radii enclosing half of the total $33 \mathrm{GHz}$ light are approximately 50 and $35 \mathrm{pc}$ for the eastern and western nucleus, respectively. Literature values of the gas mass and infrared luminosity are combined with the $33 \mathrm{GHz}$ sizes under the assumption of co-spatiality to show that Arp 220 has one of the highest molecular gas surface densities $\left(\Sigma_{\text {mol }} \sim 10^{5.3}\right.$ (east) and $10^{5.7}$ (west) $\left.\mathrm{M}_{\odot} \mathrm{pc}^{-2}\right)$ and SFR surface densities $\left(\Sigma_{\mathrm{SFR}} \sim 10^{4.0}\right.$ (east) and $10^{4.0}$ (west) $\mathrm{M}_{\odot} \mathrm{yr}^{-1} \mathrm{kpc}^{-2}$ ) measured for any star-forming system. Despite these high values, the nuclei of Arp 220 are not maximal starbursts (i.e., under the assumption that the main feedback mechanism is radiation pressure
\end{abstract}


on dust). The small derived sizes for the nuclei indicate Arp 220 is only optically thin in a narrow frequency range, $\sim 5$ to $350 \mathrm{GHz}$.

The analysis of a larger sample of $22 \mathrm{U} /$ LIRGs at $33 \mathrm{GHz}$ with the VLA is also presented. It is found that, for most of these galaxies, the integrated radio flux densities correlate well with those at infrared wavelengths, indicating these systems follow the radio-IR correlation and that the emission at $33 \mathrm{GHz}$ is primarily produced by star formation activity. The radio emission from most of these galaxies are resolved, with deconvolved half-light radii ranging from 20 pc to $1.7 \mathrm{kpc}$. Similar assumptions for Arp 220 above are used here to estimate SFR surface densities of $\Sigma_{\mathrm{SFR}}$ from $10^{0.5}$ to $10^{4.5} \mathrm{M}_{\odot} \mathrm{yr}^{-1} \mathrm{kpc}^{-2}$ and molecular gas surface densities $\Sigma_{\text {mol }}$ of $10^{2.5}$ to $10^{5.7}$ $\mathrm{M}_{\odot} \mathrm{pc}^{-2}$. These values are among the highest values measured for any galaxies. The star formation-gas scaling relation is used to compare the U/LIRGs with regions within normal spiral galaxies. The presence of two "modes" of star formation is inferred in the comparison, although this result is extremely dependent on the COto- $\mathrm{H}_{2}$ conversion factor. The local U/LIRGs studied in this survey show high infrared surface brightnesses, however 19 of the 22 sources are not maximal starbursts. Finally, those targets showing the flattest $1.5-6 \mathrm{GHz}$ spectral indices and the highest surface brightnesses exhibit the strongest [CII] deficits, which supports the idea that deficit is associated with the most highly obscured, high energy density star-forming regions.

In order to determine the true limit for star formation in galaxies (e.g., through Eddington limit analysis), better measures of the gas content, opacity and velocity dispersion of U/LIRGs are needed. The last study presented in this thesis is an analysis of the first high spatial resolution ALMA observations of the mm continuum and dense molecular gas tracers in Arp 220. A spatial resolution of $30 \mathrm{pc}$ is achieved using the most extended configuration available in Cycle 3. An optically thin model of the spectral flux density distribution is found to predict the continuum emission at 92 $\mathrm{GHz}$, within the uncertainties of the measurement and accounting for extended emission that is potentially filtered out. At $92 \mathrm{GHz}$, the western nucleus is dominated by dust emission, while the eastern nucleus by free-free emission. High critical gas density tracers $\mathrm{HCN}, \mathrm{HCO}^{+}$, their isotopologues, and the shock tracer $\mathrm{SiO}$ are detected. P-Cygni profiles are observed in the central beam of both nuclei, with a cleaner profile 
shape in the eastern nucleus. The western nucleus shows strong absorption in the center, which makes determination of the profile line shapes more complicated. These P-Cygni features indicate the presence of outflowing gas. The derived mass loading factors are 18 (east) and 35 (west), which may be an indication that active galactic nuclei help to boost the outflow mass rates. However, these numbers are strongly dependent on the highly uncertain HCN-to-gas mass conversion factor and should only be considered as upper limits. In addition to signatures of outflowing gas, clear evidence of gas rotation in both nuclei are observed. 


\section{Acknowledgements}

I could not be writing this without the support, encouragement and love of many people. Thanks to many, I am writing to express my gratitude for what it feels like the end of a long chapter of my life that started when I was little, when I first told my dad I wanted to be an Astronomer.

First, I want to thank my immediate family for always believing in me. My father, Raimundo, for encouraging me to think outside the box and to not always trust what I was taught, but always take it as something to be analyzed and then slowly internalized. My mother, Patricia, for her constant care and encouragement to go above and beyond. My siblings Paula, Patricio, Francisca, Gonzalo and Javiera for spending time teaching me when I needed to learn things they went through before I had to. My nephews Benjamin and Camilo for always being so excited to have an aunt leaving abroad and studying astronomy. My husband, Miguel, you were key for this success. Your endless support has been invaluable in this process. I thank you for always reminding me what I am capable of, for cheering me up when I was struggling, and for being my support when I got homesick (specially the first years). But most important of all, for loving our lovely daughter and for taking such a good care of her, especially in those nights when she was sick and I needed to rest so I could finish this dissertation. To my daughter, Martina, who helped me discover I was capable of things I did not know were possible and for motivating me in the last part of my PhD. You make every extra effort so worth it at the end of the day. To all of you, thank you very much for believing in me!. I love you.

But this is not a journey I started when I arrived to the U.S six years ago. I don't know I would have ended up here without the support of my math teacher in high school, Carlos Palma, who believed in me and went beyond his duties to make sure I received the proper training to succeed in college and beyond. My undergraduate advisors, Daniel Serafini and Ricardo Avila, for appreciating my hard work and supporting me even though they knew I wanted to "switch" fields (from applied physics to Astronomy). To Angel Otarola (and Vivi by default) and Al Wootten, thank you for helping and guiding me in the search of my PhD institution. I 
really do not think I would have ended up in a better place. The University of Virginia and the proximity of the astronomy department to the National Radio Astronomy Observatory (NRAO) probed to be the best place for me to learn about Astronomy and to become the scientist I am right now.

Once I started my $\mathrm{PhD}$. there were several people who I am deeply grateful. Professor Whittle for always being willing to further explain things I did not get the first time he taught them in class. Professor Majewski for teaching with that contagious passion for observational astronomy that made me enjoy much his classes. Professor Arras for teaching astrophysics in an approachable way making my first semester a bit less overwhelming. Professor Johnson for showing such commitment to outreach that made me join her efforts in teaching astronomy to local K-12 students. Jackie and Barbara, for helping me solve all sort of problems that arose along the way.

I also want to mention some of my fellow classmates and now colleagues who helped me build my knowledge and overcome difficulties along the way. I want to thank Guillermo for his endless patience and for always being willing to teach me how to code in Python considering I knew pretty much nothing about it when I first arrive to UVa. Kristen, Kim, Jo for your friendship and for always being willing to help me with my english and the many times I asked you how to pronounce certain words (I still struggle with "focus" and certainly with "ambiguous"). Aaron Kingery and Ben Breslauer for their patience and willingness to teach me when I needed help with homeworks. George, Jake and Sean, my research siblings, for great discussions.

I would like to thank people at NRAO including Crystal Brogan, Jim Condon, Amanda Kepley, John Hibbard, Todd Hunter, Eric Murphy, Erica Weller, Andrew McNichols for sharing their expertise more than once and helping me finish this chapter of my life. Special thanks to Sabrina Stierwalt for the countless supportive words and mentoring advice. You added greatness to the last years of my PhD. I also thank my collaborators for all the supportive words and useful feedback on my work. I specially want to thank Emmanuel Momjian for the endless conversations trying to understand the anomalous signals in the $6 \mathrm{GHz}$ data.

Last but not least, I want to express my deepest appreciation and gratitude to my 
advisors Adam Leroy and Aaron Evans for their constant encouragement, support, mentorship and most of all, their patience. Adam, thank you for the endless hours you dedicated teaching me how to reduce radio data, explaining me the physics behind certain processes and going over our papers making sure I was not saying crazy things. Aaron, thank you for always giving me the right advice, for teaching me the political side of being a researcher, and for sharing your wisdom and knowledge. I owe you both everything I know and the scientist I have become. Thanks for agreeing to guide me through one of the most important chapters of my life.

Thank you all for believing in me. 


\section{Table of contents}

$\begin{array}{ll}\text { Abstract } & \text { ii }\end{array}$

Acknowledgements $\quad$ v

List of Figures $\quad$ xi

List of Tables $\quad$ xii

1 General Introduction $\quad \mathbf{1}$

1.1 Importance of Major Merger-Induced Starbursts . . . . . . . . . . . . 1

1.2 The Power of Radio Continuum . . . . . . . . . . . . . . . . . 3

1.2.1 The Drawback of Radio Continuum . . . . . . . . . . . . 4

1.3 Contents of This Thesis . . . . . . . . . . . . . . . 5

2 High-Resolution Radio Continuum Measurements of the Nuclear $\begin{array}{ll}\text { Disks of Arp } 220 & 7\end{array}$

$\begin{array}{ll}\text { Abstract } & 8\end{array}$

2.1 Introduction . . . . . . . . . . . . . . . . . . . 9

2.2 Observations . . . . . . . . . . . . . . . . . . . . . . . 11

2.3 Results ............................... 13

2.3.1 Integrated Flux Densities and Spectral Indices . . . . . . . . . 15

2.3.2 Comparison to Gas and Recent Star Formation . . . . . . . . 16

2.3.3 The Morphology of Arp 220 nuclei at $33 \mathrm{GHz}$. . . . . . . . . 16

2.3.4 Brightness Temperatures . . . . . . . . . . . . 21

2.4 Discussion . . . . . . . . . . . . . . . . . . . . . . . 22

2.4.1 Synchrotron Produces Most of the $33 \mathrm{GHz}$ Emission . . . . . . 23

2.4.2 The Radio Emission Coincides with Gas, Hot Dust and RSN/SNR 25

2.4.3 The Nuclear Disks are the Most Extreme Starburst Environments in the Local Universe . . . . . . . . . . . . . . . 27

2.4.4 Evidence at Radio Wavelengths of a Dominant AGN in the Western Nucleus . . . . . . . . . . . . . . 37

2.5 Summary . . . . . . . . . . . . . . . . . 38 
Appendices $\quad 40$

2.A Flux Through An Area Just Above an Extended Thin Disk . . . . . . 41

2.B Vertical Hydrostatic Equilibrium for a Simple Radiation Pressure Dominated Disk . . . . . . . . . . . . . . . . . 43

3 A $33 \mathrm{GHz}$ Survey of Local Major Mergers 45

$\begin{array}{ll}\text { Abstract } & 46\end{array}$

3.1 Introduction . . . . . . . . . . . . . . . . . 46

3.2 Sample, Observations, and Data Reduction . . . . . . . . . . . . 49

3.2.1 Additional Data . . . . . . . . . . . . . . . 55

3.3 Results . . . . . . . . . . . . . . . . . . 55

3.3.1 Flux Densities at $\nu=32.5 \mathrm{GHz}$. . . . . . . . . . . . 57

3.3.2 Spectral Indices Involving $\nu=32.5 \mathrm{GHz}$. . . . . . . . . . . 58

3.3.3 Size of the Radio Emission . . . . . . . . . . . . . . . . . 61

3.3.4 Compact Sources Decomposition . . . . . . . . . . . . 64

3.4 Implications of the Radio Sizes . . . . . . . . . . . . . . . 66

3.4.1 Brightness Temperatures . . . . . . . . . . . . . . . 68

3.4.2 Star Formation Rate and IR Surface Density . . . . . . . . . . 70

3.4.3 Gas Surface and Column Density . . . . . . . . . . . . . 72

3.4.4 Gas Volume Density . . . . . . . . . . . . . . . . . . 74

3.5 Discussion . . . . . . . . . . . . . . . . . . . . . . 74

3.5.1 Nature of the $33 \mathrm{GHz}$ Radio Emission . . . . . . . . . . . 76

3.5.2 Physical Conditions at the Heart of Local Major Mergers . . . 80

3.5.3 The $[\mathrm{CII}]$ deficit . . . . . . . . . . . . . . . . . . . . 82

3.5.4 Implications for Star Formation Scaling Relations . . . . . . . 83

3.5.5 Are Local Major Mergers Maximal Starbursts? . . . . . . . . . 88

3.6 Conclusions . . . . . . . . . . . . . . . . . . . . . . 89

$\begin{array}{ll}\text { Appendices } & 93\end{array}$

3.A Notes on the sources . . . . . . . . . . . . . . . 94

4 ALMA High Spatial Resolution Observations of Dense Molecular $\begin{array}{ll}\text { Gas in Arp } 220 & 97\end{array}$

$\begin{array}{lr}\text { Abstract } & 98\end{array}$

4.1 Introduction . . . . . . . . . . . . . . . . . . . . . . . . . . . . . . . . 99

4.2 ALMA Observations of Arp 220 . . . . . . . . . . . . . . . . 101

4.3 Continuum and Spectral Flux Density Distribution . . . . . . . . . 107

4.4 Line Emission . . . . . . . . . . . . . . . . . . . . . . . . . . . 112

4.4.1 Absorption and P-Cygni Profiles: Dense Gas Outflowing From

the Nuclear Disks . . . . . . . . . . . . . . . . . . . . . . 113

4.4.2 Kinematics From $\mathrm{HCN}$ and $\mathrm{HCO}^{+}$Emission . . . . . . . . . . 117 
4.5 Conclusions . . . . . . . . . . . . . . . . . . . 122

4.6 Future Work . . . . . . . . . . . . . . . . . . 123

5 Summary and Future Work $\quad 126$

5.1 Summary . . . . . . . . . . . . . . . . . 126

5.2 Future Work . . . . . . . . . . . . . . . . . . 127

5.2.1 Detail spectral index maps . . . . . . . . . . . . . . . . 127

5.2 .2 Radio Recombination Lines in Arp 220 . . . . . . . . . . . . . 129

5.2 .3 Probing Radio Physics in Detail . . . . . . . . . . . . . . . . . 129

5.2.4 Gas content and kinematics in local U/LIRGs . . . . . . . . . 130

References 


\section{List of Figures}

1.1 Overwhelming dust in Arp 220 transparent to radio frequencies. . . . 2

1.2 Spectral energy distribution from a typical starburst galaxy, M 82 from Condon (1992) ............................ 5

2.1 Radio emission from Arp 220 at 6 and $33 \mathrm{GHz}$. . . . . . . . . . . . 14

2.2 Radial profiles of the $33 \mathrm{GHz}$ emission of the nuclei of Arp 220. . . . 21

2.3 Radiation pressure feedback on local U/LIRGs. . . . . . . . . . . . . 35

3.1 High spatial resolution $33 \mathrm{GHz}$ VLA continuum maps from 22 of the most luminous infrared galaxies. . . . . . . . . . . . . . . . 59

3.2 Assessment of the nature of the energy source at $33 \mathrm{GHz}$. . . . . . 60

3.3 Sizes of the radio emitting regions detected at $32.5 \mathrm{GHz}$ from our sample 63

3.4 Flux density contribution from compact sources . . . . . . . . . . 67

3.5 Brightness Temperature Histograms . . . . . . . . . . . . . . . 69

3.6 Radio-IR correlation and Histogram of $\Sigma_{\mathrm{SFR}}$ and $\Sigma_{\mathrm{IR}} \ldots \ldots . . . . .71$

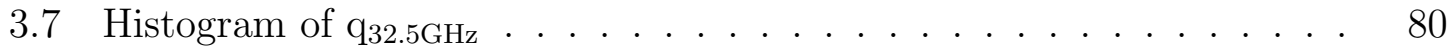

3.8 CII deficit in local U/LIRGs . . . . . . . . . . . . . . . . 83

3.9 Star formation-molecular gas scaling relation . . . . . . . . . . . . 85

3.10 Infrared surface brightness versus radius for local U/LIRGs. Assessing maximal starburst limit for local U/LIRGs. . . . . . . . . . . . . . . . 89

4.1 ALMA High-resolution continuum emission from Arp 220 at $3.3 \mathrm{~mm}$. 102

4.2 Continuum subtracted integrated spectra of each nuclei of Arp 220 . 105

4.3 Continuum subtracted spectra from central nuclear beam of each nuclei of Arp $220 \ldots \ldots \ldots$. . . . . . . . . . . . . . . . 106

4.4 Nature of the $92 \mathrm{GHz}$ emission in the nuclei of Arp 220 . . . . . . . 110

4.5 P-Cygni profiles from the central beam spectra of each nuclei . . . . . 114

4.6 High spatial resolution HCN (1-0) moment maps of Arp 220 . . . . . 118

4.7 High spatial resolution $\mathrm{HCO}^{+}(1-0)$ moment maps of Arp 220 . . . . 119

4.8 Position-Velocity diagrams of $\mathrm{HCN}$ and $\mathrm{HCO}^{+}$emission along the major axis of each nuclei. . . . . . . . . . . . . . . . . . . . . . . 121

5.1 Example of extended synchrotron emission seen at $4.7 \mathrm{GHz}$ in the galaxy UGC 08696 (Mrk 273) . . . . . . . . . . . . . . . . . 128 


\section{List of Tables}

2.1 Flux Densities and Spectral indices for Arp 220 . . . . . . . . . . . 18

2.2 Best-Fit Morphology for the Nuclei of Arp 220 at $33 \mathrm{GHz}$. . . . . . 18

2.3 Average Brightness Temperatures in Arp 220 . . . . . . . . . . . 22

3.1 Characteristics of the Sample Galaxies . . . . . . . . . . . . . 50

3.2 Summary of the Observations . . . . . . . . . . . . . . . . . . . . . . . . . . . 52

3.3 Summary of Resulting Images . . . . . . . . . . . . . . . . . . 56

3.4 Summary of $32.5 \mathrm{GHz}$ emission sizes . . . . . . . . . . . . . . 65

3.5 Summary of derived average physical parameters based on $32.5 \mathrm{GHz}$ continuum emission sizes* . . . . . . . . . . . . . . . 75

4.1 Flux densities at different frequencies for Arp 220 . . . . . . . . . . . 104

4.2 Summary of the lines detected by ALMA . . . . . . . . . . . . . . 104 


\section{Chapter 1}

\section{General Introduction}

\subsection{Importance of Major Merger-Induced Starbursts}

Luminous and ultraluminous infrared galaxies (LIRGs: $10^{11} \leq L_{I R}[8-1000 \mu \mathrm{m}]<$ $10^{12}$, ULIRGs: $L_{I R} \geq 10^{12}$ ) represent some of the most active systems in the local Universe. These galaxies are powered by extreme star formation and active galactic nuclei $(\mathrm{AGN})$. In the local universe they are primarily triggered by galaxy interactions and mergers (e.g., Sanders \& Mirabel 1996, and reference therein). Their high infrared luminosities are explained by the ultraviolet (UV) light emitted by the starburst, and/or AGN, being absorbed by obscuring dust and re-emitted at infrared wavelengths. One example of these local U/LIRGs is the prototypical merger, Arp 220 (see Figure 1.1), where high dust obscuration blocks direct view of the energetic nuclear regions.

Over the last two decades, U/LIRGs have been established as an important extragalactic population. The primary reasons are:

- U/LIRGs are essentially giant stellar nurseries - the entire molecular ISM of the progenitor galaxies has agglomerated within the central few kpc of the merger, igniting star formation rates (SFRs) up to two orders of magnitude higher than the total SFR in our galaxy (e.g., Solomon et al. 1997; Downes \& Solomon 1998; Evans et al. 2002; Iwasawa et al. 2009).

- While their local space density is low, they become much more abundant with 


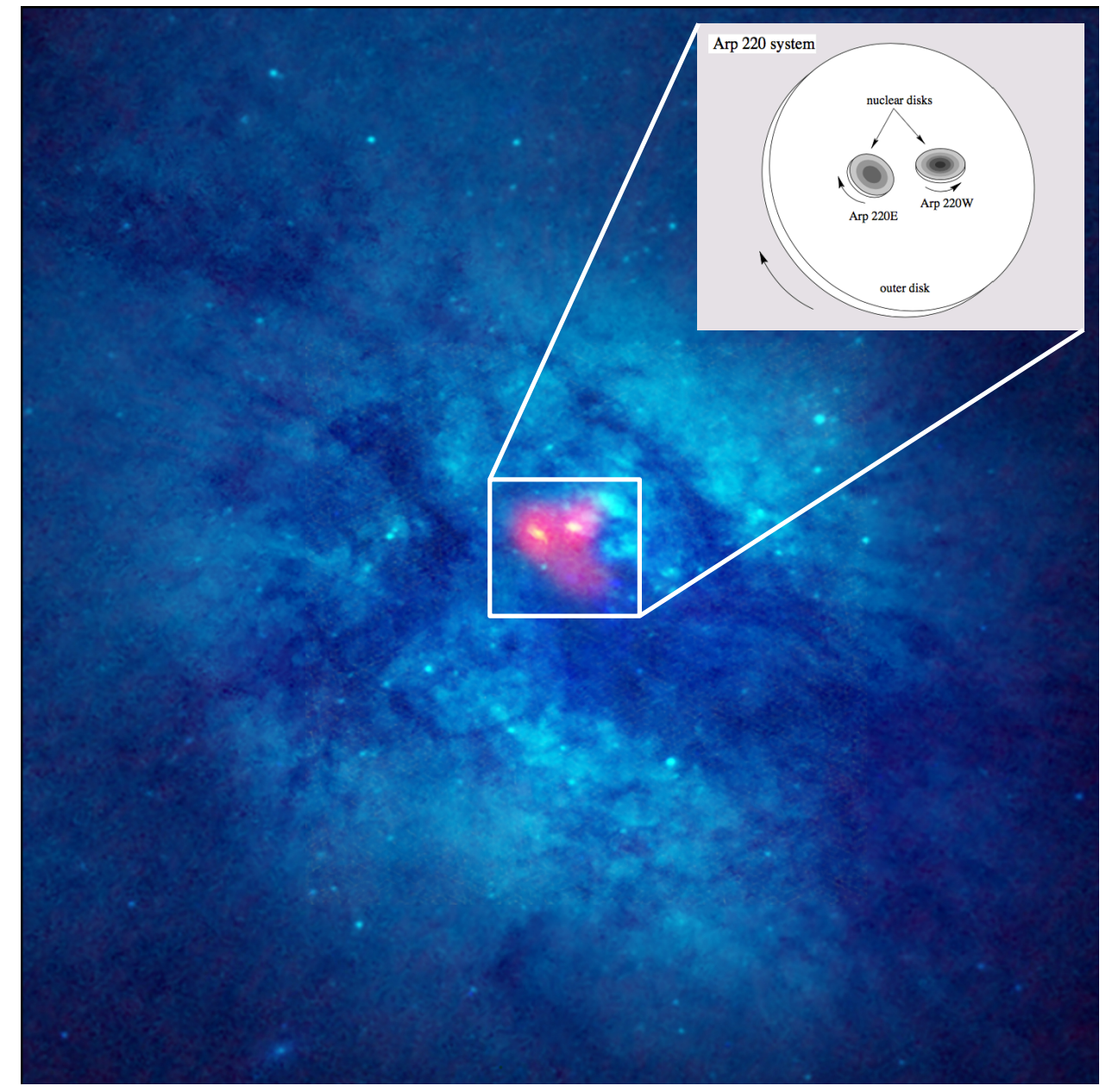

Fig. 1.1.-: HST optical image of Arp 220 ( 0.4 and $0.8 \mu \mathrm{m}$ in blue, courtesy of Aaron Evans) showing the overwhelming amounts of dust present in this system (darker filamentary patches). 6 and $33 \mathrm{GHz}$ continuum image of Arp 220 overlaid in red and yellow, respectively (Credit: B. Saxton NRAO/AUI/NSF). Inset: schematic illustration of the motion of the gas present in the nuclei of Arp 220 from Sakamoto et al. (1999).

increasing redshift $(z)$. In fact, these systems become the dominant contributor to the IR luminosity density at $z>0.5$, where the bulk of the galaxy mass build-up occurs (e.g., Le Floc'h et al. 2005; Magnelli et al. 2011). The extreme population of merger induced starbursts is present across all redshifts. Indeed, the Submillimeter Galaxies (SMGs), among the brightest systems in the universe, may be such systems (e.g., Tacconi et al. 2008).

- A variety of different diagnostics are suggestive of the presence of AGN as a significant energy source in the ultraluminous infrared galaxies (Petric et al. 2011; Iwasawa et al. 2011), where the fraction of sources hosting an AGN increases with 
the IR luminosity (e.g., Veilleux et al. 1995, 1997; Kim \& Sanders 1998), making these systems ideal for studying the connection between AGN and starburst activity. This, in addition to their high bolometric luminosities (comparable to optically selected QSOs), opens the possibility of an evolutionary model connecting QSOs to ULIRGs (Sanders et al. 1988b,a).

The extreme environments seen in these major mergers makes them ideal for studying different mechanisms regulating the star formation rate and stellar clustering in regimes very different from the Milky Way. The intense starbursts in these mergers often occur in regions of a few hundred parsecs in size. The lack of high-resolution, resolved maps of recent star formation, gas, and kinematics has limited our ability to assess the properties of merger-driven star formation and feedback processes at play. Examples of proposed theories of merger-driven star formation include radiative feedback (Scoville 2003; Thompson et al. 2005), feedback from momentum-driven winds (Murray et al. 2005), turbulence (Krumholz \& McKee 2005a), and collisions between bound structures (Tan 2000; Tan et al. 2009). The local surface density of star formation is an important ingredient to tests of these theories.

\subsection{The Power of Radio Continuum}

Multiwavelength observations are a useful method to obtain crucial information on the star formation distribution within these sources. Indeed, the process responsible for the creation of their high infrared luminosities, the absorption of UV light by obscuring dust have made them difficult to study at optical and UV wavelengths. Further, while the bulk of their bolometric luminosity is observed at infrared wavelengths, infrared telescopes to date (e.g., IRAS, ISO, and Spitzer) have insufficient resolution to resolve the energetically dominant regions of even the nearest U/LIRGs. This constitutes a fundamental challenge to understanding these systems, because the resolved distribution of activity (especially the star formation surface density, $\Sigma_{\mathrm{SFR}}$ ) is a crucial property for both the appearance and evolution of these sources.

Radio continuum (RC) offers a powerful alternative to IR wavelengths for the following reasons: a) the emission in the centimeter-to-millimeter wavelengths regime 
does not suffer extinction due to dust, b) the nature of the emission is a combination of free-free (produced by the acceleration of free electrons by the interaction with ions in an ionized medium, such as ionized Hydrogen (HII) regions surrounding newly formed stars), synchrotron (produced by the acceleration of ultrarelativistic electrons by magnetic fields, such as in the envelopes of supernovae explosions) and thermal dust radiation (see Figure 1.2 obtained from Condon (1992)). The first two processes trace emission associated with star formation; and, c) unlike IR telescopes to date, radio interferometers achieve high angular resolution (sub-arcsecond). Mapping the $\mathrm{RC}$ emission from LIRGs at high resolution represents one of the only ways to assess the true distribution of star formation on small angular scales in the presence of overwhelming optical/IR extinction (see Figure 1.1). This has made radio interferometry one of the best ways to study the structure of compact, embedded starbursts (e.g., see review by Lonsdale et al. 2006b).

\subsubsection{The Drawback of Radio Continuum}

Unlike infrared emission, RC is not a bolometric tracer. Radio carries away only a small fraction of the total power from a starburst and is linked indirectly to star formation by a complex string of physical processes (supernovae, cosmic ray electron acceleration, photoionization, and $B$ fields; see Condon 1992). Our confidence that the RC traces star formation rests largely on an empirical correlation, the RC-far infrared correlation. Most work on this correlation has been done for whole galaxies at (only) one wavelength, 20 cm (e.g., Condon et al. 1991a; Yun et al. 2001). However, we know that the RC-IR relation exhibits complex environmental dependencies within galaxies (e.g., Dumas et al. 2011; Tabatabaei et al. 2013) and that the radio spectral energy distributions (SEDs) of starburst galaxies can be complex (Condon et al. 1991b; Clemens et al. 2010; Leroy et al. 2011; Murphy et al. 2013). This complicates a simple interpretation equating monochromatic radio intensity with $\Sigma_{\mathrm{SFR}}$ and suggests the need of a more sophisticated view of radio emission. This thesis is the best attempt to date of a more sophisticated view. 


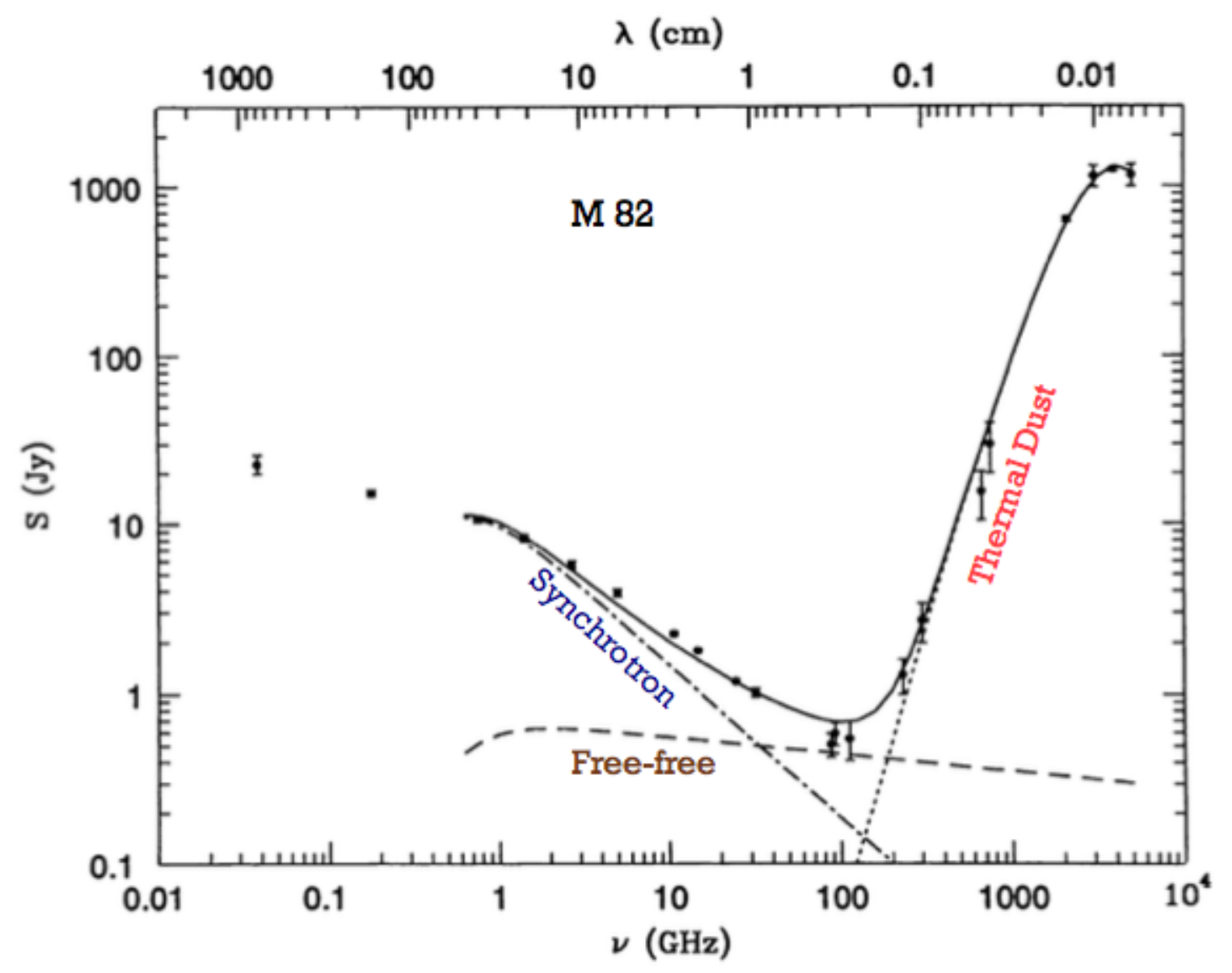

Fig. 1.2.- : Observed spectral energy distribution from a normal starburst galaxy, Messier 82, from Condon (1992). Three main components contribute to the SED in the centimeter/millimeter regime, free-free (negative flat spectrum shown by the dashed line), synchrotron (negative steep spectrum shown by the dotted-dashed line, and thermal dust emission (positive steep spectrum shown by the dotted line). The circles show the observed flux density (y axis) at different frequencies (x axis) and the solid line is the sum of the emission modeled by the three components.

\subsection{Contents of This Thesis}

This thesis is mainly focused on studying star formation, and the associated mechanisms, in a sample of U/LIRGs from the IRAS Revised Bright Galaxy Sample $\left(f_{60 \mu m} \gtrsim 5.24\right.$ Jy; Sanders et al. 2003). This work makes use of the first highresolution multi radio frequency survey of U/LIRGs using the upgraded Very Large Array. In Chapter 2, the results from a detailed analysis of this radio survey performed on the prototypical U/LIRG, Arp 220, are presented. This Chapter was published in the Astrophysical Journal in January of 2015. In Chapter 3, the high spatial resolution results of the survey including the entire sample of local U/LIRGs is 
reported (22 systems in total). This Chapter will be submitted to the Astrophysical Journal by the beginning of the Fall semester this year. In Chapter 4, the primary focus is the dense molecular gas content in Arp 220 as seen by high spatial resolution observations made with the Atacama Large Millimeter/submillimeter Array (ALMA) in one of the first attempts to study the star-forming gas properties at $30 \mathrm{pc}$ resolution in this galaxy. Chapter 5 is a summary of the work done in this thesis and a description of planned future efforts to understand star formation in local U/LIRGs. For the presented work, I was in charge of the full data reduction and analysis of the data, and the initial drafts of the Chapters were written by me. Finally, I was the PI of the ALMA proposal which resulted in the Arp 220 dataset presented in Chapter 4 . 
Chapter 2

High-Resolution Radio Continuum Measurements of the Nuclear Disks of Arp 220 


\begin{abstract}
We present new Karl G. Jansky Very Large Array radio continuum images of the nuclei of Arp 220, the nearest ultra-luminous infrared galaxy. These new images have both the angular resolution to study the detailed morphologies of the two nuclei that power the galaxy merger and sensitivity to a wide range of spatial scales. At $33 \mathrm{GHz}$, we achieve a resolution of $0^{\prime \prime} .081 \times 0^{\prime \prime} .063(29.9 \times 23.3 \mathrm{pc})$ and resolve the radio emission surrounding both nuclei. We conclude from the decomposition of the radio spectral energy distribution that a majority of the $33 \mathrm{GHz}$ emission is synchrotron radiation. The spatial distributions of radio emission in both nuclei are well-described by exponential profiles. These have deconvolved half-light radii $\left(\mathrm{R}_{50 \mathrm{~d}}\right)$ of 51 and $35 \mathrm{pc}$ for the eastern and western nuclei, respectively, and they match the number density profile of radio supernovae observed with very long baseline interferometry. This similarity might be due to the fast cooling of cosmic rays electrons caused by the presence of a strong $(\sim \mathrm{mG})$ magnetic field in this system. We estimate extremely high molecular gas surface densities of $2.2_{-1.0}^{+2.1} \times 10^{5}$ (east) and $4.5_{-1.9}^{+4.5} \times 10^{5}$ (west) $\mathrm{M}_{\odot} \mathrm{pc}^{-2}$, corresponding to total hydrogen column densities of $\mathrm{N}_{\mathrm{H}}=2.7_{-1.2}^{+2.7} \times 10^{25}$ (east) and $5.6_{-2.4}^{+5.5} \times 10^{25} \mathrm{~cm}^{-2}$ (west). The implied gas volume densities are similarly high, $\mathrm{n}_{\mathrm{H}_{2}} \sim 3.8_{-1.6}^{+3.8} 10^{4}$ (east) and $\sim 11_{-4.5}^{+12} \times 10^{4} \mathrm{~cm}^{-3}$ (west). We also estimate very high luminosity surface densities of $\Sigma_{\mathrm{IR}} \sim 4.2_{-0.7}^{+1.6} \times 10^{13}$ (east) and $\Sigma_{\mathrm{IR}} \sim 9.7_{-2.4}^{+3.7} \times 10^{13}$ (west) $\mathrm{L}_{\odot} \mathrm{kpc}^{-2}$, and star formation rate surface densities of $\Sigma_{\mathrm{SFR}} \sim 10^{3.7 \pm 0.1}$ (east) and $\Sigma_{\mathrm{SFR}} \sim 10^{4.1 \pm 0.1}$ (west) $\mathrm{M}_{\odot} \mathrm{yr}^{-1} \mathrm{kpc}^{-2}$. These values, especially for the western nucleus are, to our knowledge, the highest luminosity surface densities and star formation rate surface densities measured for any star-forming system. Despite these high values, the nuclei appear to lie below the dusty Eddington limit in which radiation pressure is balanced only by self-gravity. The small measured sizes also imply that at wavelengths shorter than $\lambda=1 \mathrm{~mm}$, dust absorption effects must play an important role in the observed light distribution while below $5 \mathrm{GHz}$ freefree absorption contributes substantial opacity. According to these calculations, the nuclei of Arp 220 are only transparent in the frequency range $\sim 5$ to $350 \mathrm{GHz}$. Our results offer no clear evidence that an active galactic nucleus dominates the emission
\end{abstract}


from either nucleus at $33 \mathrm{GHz}$.

\section{$2.1 \quad$ Introduction}

Starbursts induced by major mergers are among the most extreme environments in the universe. Despite their prodigious luminosities, local merger-driven starbursts are very compact, with most of their large gas reservoirs concentrated in dusty regions a few hundred parsecs, or less, in size (e.g., Downes \& Solomon 1998). Measuring the compactness of these starbursts is critical to understanding these galaxies (e.g., Soifer et al. 1999, 2000; Sakamoto et al. 2008; Díaz-Santos et al. 2013). Robust size measurements allow us to translate luminosities into key physical quantities such as gas column density, optical depth, volumetric gas density, and star formation rate and luminosity surface densities. Although their luminosity renders them visible out to great distances, the present-day rarity of major mergers means that even the nearest ultraluminous infrared galaxies (ULIRGs: defined as having $\mathrm{L}_{\mathrm{IR}}[8-1000 \mu \mathrm{m}] \geq 10^{12} \mathrm{~L}_{\odot}$ ) are relatively distant $(>70 \mathrm{Mpc})$. Thus, measuring the true extent of their active regions requires high angular resolution. The extraordinary extinctions present in these systems at both long (from free-free absorption) and short (from dust opacity) wavelengths complicate the interpretation of measurements at both wavelengths, compounding the difficulty of measuring sizes for such systems.

Given the above considerations, radio observations at centimeter wavelengths may be the best tool to study the deeply embedded, compact structures at the heart of such systems (e.g., Norris 1988; Condon et al. 1991b). Radio interferometers can achieve very high angular resolution and radio waves with $\nu \gtrsim 5 \mathrm{GHz}$ can penetrate large columns of dust and are largely unaffected by free-free absorption. The recent upgrades to the Karl G. Jansky Very Large Array (VLA) make it particularly wellsuited for such studies. In this paper, we make use of these new VLA capabilities to achieve the best measurement to date of the structure of the nuclear region of the nearest ULIRG, Arp 220.

At a luminosity distance of $77.2 \mathrm{Mpc}$, and an infrared luminosity of $\mathrm{L}_{\mathrm{IR}}[8-1000 \mu \mathrm{m}]$ 
$=1.44 \times 10^{12} \mathrm{~L}_{\odot}{ }^{1}$, Arp 220 is the nearest ULIRG. CO and near-IR observations indicate that Arp 220 is a gas rich merger with dynamical masses of $\sim 10^{9} \mathrm{M}_{\odot}$ within $\sim 100$ pc of each nucleus (Downes \& Solomon 1998; Sakamoto et al. 1999; Genzel et al. 2001; Sakamoto et al. 2008; Engel et al. 2011). Arp 220 is obscured at optical through mid-IR wavelengths (Scoville et al. 1998; Soifer et al. 1999; Haas et al. 2001; Spoon et al. 2007; Armus et al. 2007), obstructing the direct view of the nuclear energy sources at these wavelengths. Observations in the frequency range where Arp 220 is optically thin have been able to resolve the system into two compact nuclear disks (Norris 1988; Condon et al. 1991b; Downes \& Solomon 1998; Sakamoto et al. 2008) and find disk sizes of $\sim 0{ }^{\prime} 2$. However, in each case the measured sizes remain comparable to the size of the beam. VLBI observations at cm wavelengths by Smith et al. (1998), Lonsdale et al. (2006a), and Parra et al. (2007) provide a higher resolution view, recovering a compact distribution of point-like sources that are proposed to be a combination of radio supernovae (RSNe) and supernova remnants (SNRs). However, these observations resolve out most of the emission from the disks. From the above, it is already clear that the disks are very compact, implying extraordinary volume densities and surface densities. The next step is to observe the disks in the optically thin frequency range with resolution high enough to clearly resolve them, and sensitivity to recover the full extent of the emission of the system at that frequency range.

In this paper, we measure the structure of the Arp 220 nuclei with sensitive, high angular resolution images obtained at $6 \mathrm{GHz}$ and $33 \mathrm{GHz}$ observed with the VLA. Based on the integrated spectral energy distribution (SED) model of Arp 220 (see Figure 10b in Anantharamaiah et al. 2000), the total continuum flux density at 33 $\mathrm{GHz}$ is a mixture of thermal and non-thermal emission with a $\sim 1: 2$ ratio, while at 6 $\mathrm{GHz}$ this ratio is about 1:5. Observing at these two frequencies then helps us diagnose the dominant emission mechanism at radio wavelengths in Arp 220. We first report our observations, describe the calculations used to assess the disk structure, and then

\footnotetext{
${ }^{1} \mathrm{D}_{\mathrm{L}}$ from NED; $\mathrm{L}_{\mathrm{IR}}$ using Table 1 in Sanders \& Mirabel (1996) and IRAS flux densities from Sanders et al. (2003). However, note that the assumption of isotropic emission that leads to this luminosity has some caveats (see Section 2.4.3 and 2.A).
} 
discuss the implications of our measurements. Throughout this paper, we adopt $\mathrm{H}_{0}=73 \mathrm{~km} \mathrm{~s}^{-1} \mathrm{Mpc}^{-1}, \Omega_{\text {vacuum }}=0.73, \Omega_{\text {matter }}=0.27$ and $\mathrm{v}_{\text {optical }}=5,555 \mathrm{~km} \mathrm{~s}^{-1}$ (after correction to the CMB frame), such that $1^{\prime \prime}$ on the sky plane subtends $369 \mathrm{pc}$ at the distance of Arp 220.

\subsection{Observations}

We observed Arp 220 using the VLA C (4-8 GHz) and Ka band (26.5-40 GHz) receivers, recording emission in $1 \mathrm{GHz}$ wide windows centered at $\sim 4.7,7.2,29$ and $36 \mathrm{GHz}$. We used all four VLA configurations with a total integration time ratio of 1:1:2:4 between D (lowest resolution), C, B, and A. The total on-source integration time was $40 \mathrm{~min}$ at $\mathrm{C}$ band and $56 \mathrm{~min}$ at Ka band. We used 3C 286 as the flux density and bandpass calibrator, and J1513+2338 and J1539+2744 as the complex gain calibrators at $\mathrm{C}$ and Ka bands, respectively. The data were obtained in multiple observing sessions during the period 2010 August 18 to 2011 July 2, with the C and Ka band observations carried out in separate sessions. These observations are part of a larger project; the lower resolution ( $\mathrm{C}$ and $\mathrm{D}$ configuration) results are presented in Leroy et al. (2011) and Murphy (2013), and the final results for the complete sample will be reported in Barcos-Muñoz et al. (in prep.).

We reduced the data using the Common Astronomy Software Application (CASA, McMullin et al. 2007) package following the standard procedure for VLA data. Radio frequency interference $(\mathrm{RFI})$ contaminating the $\mathrm{C}$ band was eliminated using the task flagdata in mode rflag. The RFI at Ka band was negligible. After calibration, we combined the data from all configurations, weighting them in proportion to their integration time per visibility (i.e., 10:5:2:1 for D:C:B:A). We then imaged this combined data using the task CLEAN in mode mfs (Sault \& Wieringa 1994), with Briggs weighting setting robust=0.5. We combined all the data within each receiver band and cleaned using components with a variable spectral index (nterms=2) to obtain an interpolated image at an intermediate frequency $(5.95 \mathrm{GHz}$ for $\mathrm{C}$ band and $32.5 \mathrm{GHz}$ for Ka band). Even after the initial calibration, we still observed phase and amplitude variations with time. To improve the images further, we iteratively 
self-calibrated in both phase and amplitude and applied extra flagging during this procedure as needed. The solutions for the amplitude self-calibration were carefully inspected and accepted as long as the time variations in the amplitude gains for each antenna were less than $\sim 20 \%$. We worked mostly with these "combined" images at 5.95 and $32.5 \mathrm{GHz}$, but we also separately imaged the two $1 \mathrm{GHz}$ windows at $\mathrm{C}$ band in order to derive a robust internal $\mathrm{C}$ band spectral index.

To check our results, we imaged the $33 \mathrm{GHz}$ data separately for each VLA configuration. In this test, we primarily applied iterative phase self-calibration. Amplitude self-calibration was applied (after $\sim 3-5$ iterations of phase self-calibration), but here we only derived normalized solutions that cannot change the observed flux. During this check, we also experimented with weighting the visibilities by the measured rms noise in each data set (using the CASA task statwt). These tests revealed that the combined $\mathrm{A}+\mathrm{B}$ (two most extended) configurations recovered essentially all of the flux in the data, agreeing with the $\mathrm{C}$ and $\mathrm{D}$ data in both flux and morphology when convolved to matched resolution. The A configuration data alone recovered less flux than the B configuration, consistent with some spatial filtering at this highest resolution. Further, the overall flux recovered agrees with an interpolation of the integrated SED (Anantharamaiah et al. 2000). We proceed using the full combined image with our confidence in the results reinforced by these tests; we verified that our fitting yields consistent results using the combined image and the $\mathrm{A}+\mathrm{B}$ configuration-only image.

The clean restoring beam for the combined images has a FWHM of 0.'48 $\times 0$ ".35 $(177 \times 129 \mathrm{pc})$ at position angle (p.a.) of $-40^{\circ}$ at $6 \mathrm{GHz}(\mathrm{C}$ band $)$, and $0^{\prime \prime} .081 \times 00^{\prime \prime} .063$ $(30 \times 23 \mathrm{pc})$ at a p.a. of $65^{\circ}$ at $32.5 \mathrm{GHz}$ (Ka band). The rms noise measured from signal-free parts of the image is $\sim 14 \mu \mathrm{Jy}_{\text {beam }^{-1}}(\mathrm{C})$ and $\sim 23 \mu \mathrm{Jy}$ beam $^{-1}$ (Ka), which is within a factor of two of the expected theoretical noise. The final dynamic ranges of the images are $\sim 5.2 \times 10^{3}$ and $\sim 280$, for $\mathrm{C}$ and Ka band.

When reporting the measured flux densities from the final images, we consider three sources of uncertainty. First, we propagated the beam-to-beam noise (see above) and found its effect to be negligible at both bands. Second, we assessed the impact of the curve-of-growth technique used to measure the flux densities (see Section 2.3) 
by using the scatter of such a curve. This is also small, but larger for the individual nuclei because they are not perfectly separable. Finally, we estimated the uncertainty in the overall flux density calibration from the day-to-day variation of the flux density of the complex gain calibrator. This scatter $(\mathrm{rms})$ is $\sim 12 \%$ at Ka band, making the flux density calibration the dominant source of uncertainty at this band. At C band, the scatter in the curves of growth and the variation in the flux density calibration are comparable, i.e., $\sim 1 \%$. We sum all three uncertainty terms in quadrature and report the combined value in Table 2.1. Note that in addition to these uncertainties, the absolute flux scale used at the VLA is estimated to be uncertain by $\approx 2 \%$.

After reducing the data, we compared our $33 \mathrm{GHz}$ image to VLA (Norris 1988; Condon et al. 1991b), SMA (Sakamoto et al. 2008) and ALMA archival images (Wilson et al. 2014; Scoville et al. 2015). We found astrometric discrepancies of order $0{ }^{\prime \prime} 1$ (i.e., 1-2 beams) and traced the origin of these to the adopted position of our $33 \mathrm{GHz}$ phase calibrator. When revised from the nominal VLA position to the position reported in the VLBI calibrator catalog, the astrometric agreement between our image and the other images improved to a fraction of a beam. For reference, in our data the peak positions of the two nuclei at $33 \mathrm{GHz}$ are $\alpha_{2000}=15^{\mathrm{h}} 34^{\mathrm{m}} 57.291^{\mathrm{s}} \pm 0.003^{\mathrm{s}}\left( \pm 0^{\prime \prime} .05\right)$, $\delta_{2000}=23^{\circ} 30^{\prime} 11^{\prime \prime} .34 \pm 0^{\prime \prime} .04$ (east) and $\alpha_{2000}=15^{\mathrm{h}} 34^{\mathrm{m}} 57.222^{\mathrm{s}} \pm 0.002^{\mathrm{s}}\left( \pm 0^{\prime \prime} \cdot 03\right), \delta_{2000}=$ $23^{\circ} 30^{\prime} 11^{\prime \prime} .51 \pm 0^{\prime \prime} .03$ (west). We derive these position via a Gaussian fit (CASA's imf it) but they also closely coincide with the positions of the highest intensity pixel for each nucleus. The uncertainties in the peak positions above may also be viewed as our overall astrometric uncertainty, which we derive from the standard deviation between the positions of the highest intensity pixels from our $6 \mathrm{GHz}$ image and our shifted $33 \mathrm{GHz}$ image, and the archival images from Norris (1988), Condon et al. (1991b), Sakamoto et al. (2008), Wilson et al. (2014) and Scoville et al. (2015).

\section{$2.3 \quad$ Results}

In Figures 2.1 and 2.2, we present new VLA images of Arp 220 at 6 and $33 \mathrm{GHz}$ and the radial profiles of each nucleus at $33 \mathrm{GHz}$. Using these new data, combined with a $\mathrm{CO}(3 \rightarrow 2)$ integrated intensity ("zeroth moment") map from Sakamoto et al. 

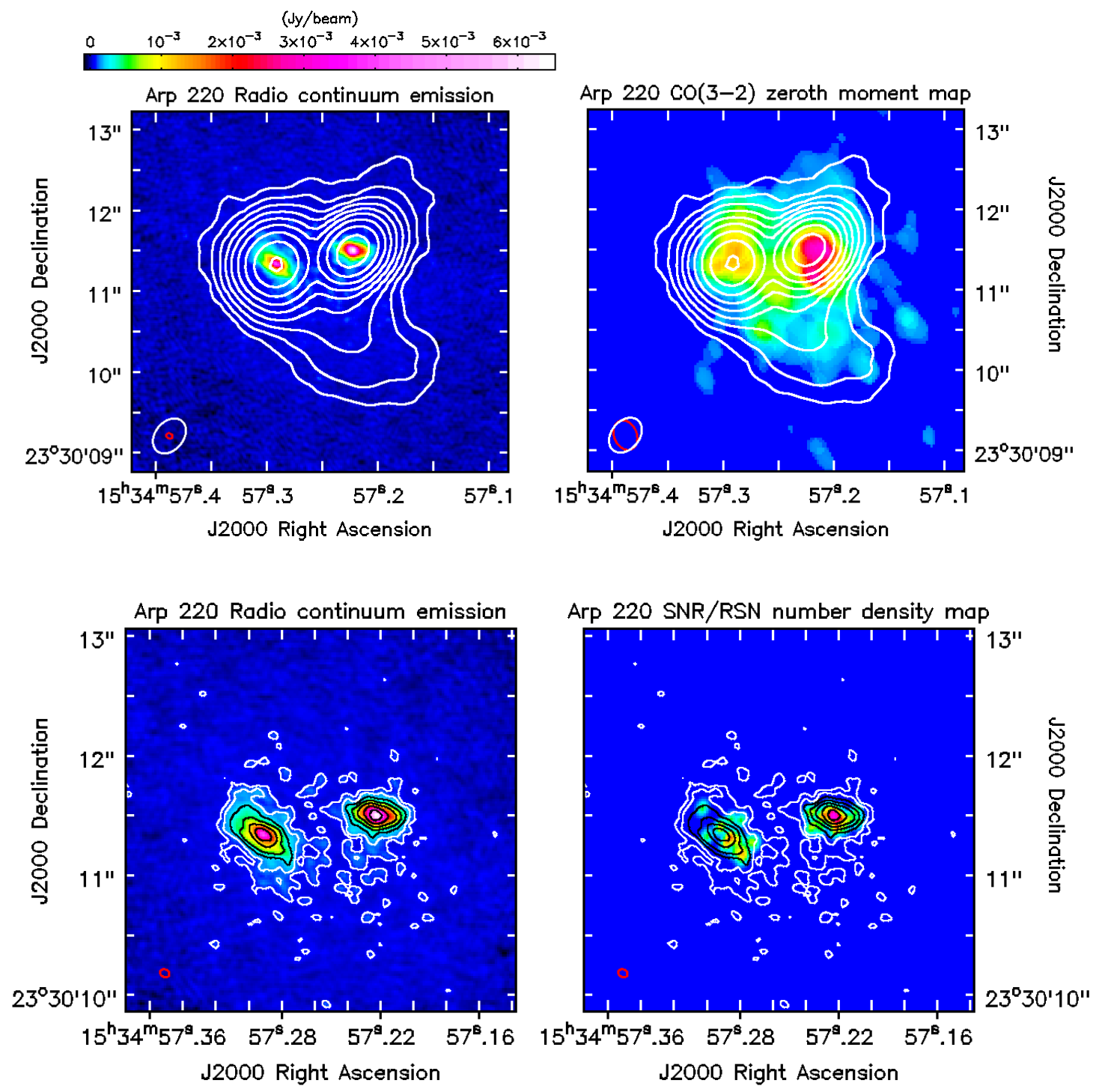

Fig. 2.1.—: (a) $33 \mathrm{GHz}$ image (FWHM resolution of $0 !^{\prime \prime} 081 \times 00^{\prime \prime} 063$, p.a. $\approx 65^{\circ}$ ) with $6 \mathrm{GHz}$ contours overlaid (FWHM resolution of $0 !^{\prime \prime} 48 \times 00^{\prime \prime} 35$, p.a. $\approx-40^{\circ}$ ). The contours are in factor of 2 step, with the outermost contour corresponding to $12.5 \sigma$, where $\sigma=14 \mu \mathrm{Jy}_{\text {beam }}{ }^{-1}$, and enclosing $98 \%$ of the total flux density. In the lower left corner, we show the FWHM of the clean beam for the $33 \mathrm{GHz}$ (red) and $6 \mathrm{GHz}$ (white) image. (b) The same $6 \mathrm{GHz}$ contours overlaid on the $\mathrm{CO}(3 \rightarrow 2)$ integrated intensity map of Sakamoto et al. (2008) (FWHM resolution of $00^{\prime \prime} 38 \times$ $0^{\prime \prime} 28$, p.a. $\left.\approx 23^{\circ}\right)$. In the lower left corner, we show the FWHM of the clean beam for the $33 \mathrm{GHz}(r e d)$ and $\mathrm{CO}(3 \rightarrow 2)$ (white) image.(c) A $33 \mathrm{GHz}$ radio continuum image with its contours overlaid. The contours are in factor of 2 step, with the outermost contour corresponding to $3 \sigma$, where $\sigma=23 \mu \mathrm{Jy}$ beam ${ }^{-1}$, and enclosing $97 \%$ of the total flux density. (d) $33 \mathrm{GHz}$ contours overlaid on a radio supernova (RSN) and/or supernova remnant (SNR) number density map constructed using the point sources found by Lonsdale et al. (2006a). We clearly resolve structure surrounding both nuclei and see a broad similarity between the radio continuum, gas traced by CO, and recent RSNe and/or SNRs.

(2008), and positional information of point sources found by Lonsdale et al. (2006a), we carry out a series of calculations to determine what mechanism is producing most 
of the radio emission, how radio emission traces recent star formation, and the true sizes and shapes of the nuclear disks. In Tables 2.1, 2.2 and 2.3 we present the results of these calculations.

\subsubsection{Integrated Flux Densities and Spectral Indices}

In Table 2.1, we report the flux density of the entire system, each nucleus, and the resulting spectral indices. We used a curve of growth method to derive the flux densities. For the integrated flux density, we progressively $(u, v)$ tapered and re-imaged the data, recording the total flux density above a signal-to-noise of 5 at each resolution. These flux densities agree with those measured from the imaging of individual arrays (see Section 2.2). For the flux densities of the individual nuclei, we used CASA's imstat task to place circular apertures around each component, varying their radii. We plotted the flux density against aperture radius and looked for convergence in this curve-of-growth to identify the true flux density. We also independently measured the integrated flux of the south-west component seen at $\mathrm{C}$ band (see top-left panel in Figure 2.1), using an aperture in the CASA viewer, and found that it encloses $\sim 3 \%$ of the total $5.95 \mathrm{GHz}$ flux density. The integrated flux densities at both frequencies agree, within the reported errors, with predicted values based on the modeled integrated SED published in the literature (e.g., Anantharamaiah et al. 2000).

Using these flux densities, we calculated the spectral indices, $\alpha$ for $\mathrm{F}_{\nu} \propto \nu^{\alpha}$, of the whole system and each nucleus via

$$
\alpha_{1-2}=\frac{\log F_{1}-\log F_{2}}{\log \nu_{1}-\log \nu_{2}},
$$

where $F_{1}$ and $F_{2}$ are the flux densities at frequencies $\nu_{1}$ and $\nu_{2}$. Equation 2.1 will be valid for flux densities $F_{1}$ and $F_{2}$ over matched apertures (or for integrated values over whole systems). 


\subsubsection{Comparison to Gas and Recent Star Formation}

To assess the degree to which the measured sizes are characteristic of the whole system, we compared our maps to known distributions of emission from gas and recent radio supernovae and/or supernova remnants (RSNe/SNRs). In the top-right panel in Figure 2.1, we plot $\mathrm{C}$ band contours over the CO $(3 \rightarrow 2)$ map of Sakamoto et al. (2008) (restoring Gaussian beam with FWHM $0^{\prime \prime} .38 \times 00^{\prime \prime} .28$ at a p.a $\approx 23^{\circ}$ ).

The RSNe/SNRs trace recent star formation, and they can accelerate cosmic ray (CR) electrons that emit synchrotron radiation. We built a map of RSN/SNR number density from the locations of 49 point sources identified by Lonsdale et al. (2006a) from $18 \mathrm{~cm}$ VLBI observations. On our $33 \mathrm{GHz}$ astrometric grid, we convolved delta functions with a fixed, fiducial intensity at the positions of the point sources with our $33 \mathrm{GHz}$ beam ${ }^{2}$. In the bottom panels of Figure 2.1, we compare the $33 \mathrm{GHz}$ map to the distribution of recent RSNe/SNRs.

Note that although we use RSNe/SNRs as signposts of recent star formation, the VLBI sources do not contribute significantly to the flux that we observe. For a typical synchrotron spectral index $\alpha_{1.7-33 \mathrm{GHz}}=-0.7$, the Lonsdale et al. (2006a) RSNe/SNRs would contribute $1.5 \mathrm{mJy}$ at $33 \mathrm{GHz}$ and $4.9 \mathrm{mJy}$ at $6 \mathrm{GHz}$. This contribution would only account for $\sim 2.5 \%$ of the total flux density that we observe with the VLA. Even at $18 \mathrm{~cm}$, the Lonsdale et al. (2006a) RSNe/SNRs have integrated flux only $12 \mathrm{mJy}$ at $18 \mathrm{~cm}$, or $\sim 4 \%$ of the total flux density of Arp 220 at that frequency (Williams \& Bower 2010). This contribution is small compared to the $10 \%$ expected fraction in normal spiral galaxies like M31 or the Milky Way (Pooley 1969; Ilovaisky \& Lequeux 1972). This difference is most likely due to free-free absorption at $18 \mathrm{~cm}$ (see Section 2.4.3).

\subsubsection{The Morphology of Arp 220 nuclei at $33 \mathrm{GHz}$}

The smooth, ellipsoidal isophotes in Figure 2.1 suggest a disk-like geometry. We modeled the $3 \sigma$ clipped image of Arp 220 at $33 \mathrm{GHz}$ (outermost contour in bottom

\footnotetext{
${ }^{2}$ Lonsdale et al. (2006a) report offsets from the center of Arp 220, which we take to be $\alpha_{2000}=$ $15^{\mathrm{h}} 34^{\mathrm{m}} 57.259^{\mathrm{s}}, \delta_{2000}=23^{\circ} 30^{\prime} 11^{\prime \prime} .409$.
} 
left panel of Figure 2.1) using a 2-D non-linear least-squares fitting technique. After experimenting with Gaussian, exponential, Sérsic and hybrid profiles, we found that the two disks are reasonably described by thin, tilted exponential disks. We fit both nuclei simultaneously by varying, without constraints, the amplitude, position angle (p.a.), inclination, center, and scale length of each nucleus. Although the parameters did not have constraints, the starting points were educated guesses of the final parameters. In each case, we construct the model image, convolve it with the synthesized beam of our observations, and compare the model and observed intensities to derive $\chi^{2}$. Note that the results for the inclination of the disks represent lower limits because we assume thin disks.

The best fit parameters from the model fitting, along with associated uncertainties, are reported in Table 2.2. In addition to deriving formal uncertainties, we gauge the accuracy of our fit by varying our approach among several reasonable methods. For example, we adopt a logarithmic, rather than linear, goodness of fit statistic and we fit the radial profile rather than the image itself. These imply an uncertainty of $\approx 15 \%$ for the scale length and a few percent for p.a. and inclination. The error in the normalization is dominated by our overall uncertainty in the amplitude calibration $(\approx 12 \%)$. As another point of comparison, we also report the results of simple Gaussian fitting, although, we emphasize that the residuals are substantially poorer for this approach at low and high radius.

From the exponential model, we obtain deconvolved scale lengths of 30 and $21 \mathrm{pc}$ for the east and west nucleus, respectively. Our results for p.a., inclination, and center did not vary significantly with the choice of functional form. While the fits appear to be good descriptions, they are not perfect. From the residual images, we found that the western nucleus showed higher residuals in the disk than the eastern nucleus, while the center of the eastern nucleus had higher residuals than the western nucleus.

Both nuclei are well resolved, showing significant extent compared with the synthesized beam. The implied deconvolved half-light radii, $\mathrm{R}_{50 \mathrm{~d}}$, are 51 and $35 \mathrm{pc}$, respectively; that is, if viewed face-on, we would expect half the emission from Arp 220 to come from nuclear disks $\sim 100$ (east) and $\sim 70$ (west) pc across. In Fig- 
Table 2.1. Flux Densities and Spectral indices for Arp 220

\begin{tabular}{|c|c|c|c|c|c|}
\hline \multirow{2}{*}{$\begin{array}{l}\text { Frequency } \\
\quad(\mathrm{GHz})\end{array}$} & \multirow{2}{*}{$\begin{array}{l}\text { Total } \\
(\mathrm{mJy})\end{array}$} & \multicolumn{2}{|c|}{ East nucleus } & \multicolumn{2}{|c|}{ West nucleus } \\
\hline & & Integrated (mJy) & Peak $\left(\mathrm{mJy} \mathrm{beam}^{-1}\right)^{\mathrm{a}}$ & Integrated (mJy) & Peak $\left(\mathrm{mJy} \text { beam }^{-1}\right)^{\mathrm{a}}$ \\
\hline 4.7 & $222.0 \pm 1.9$ & $92.4 \pm 2.1$ & $61.8 \pm 0.5$ & $114.6 \pm 4.3$ & $89.5 \pm 0.7$ \\
\hline 7.2 & $171.4 \pm 2.3$ & $73.2 \pm 1.4$ & $36.0 \pm 0.4$ & $89.5 \pm 1.4$ & $60.4 \pm 0.7$ \\
\hline 5.95 & $197.6 \pm 2.8$ & $81.4 \pm 2.8$ & $49.0 \pm 0.7$ & $94.3 \pm 1.7$ & $73.3 \pm 1.0$ \\
\hline 32.5 & $61.8 \pm 7.2$ & $30.1 \pm 3.9$ & $4.1 \pm 0.5$ & $33.4 \pm 4.0$ & $6.5 \pm 0.8$ \\
\hline \multicolumn{6}{|c|}{ Spectral index } \\
\hline$\alpha_{6-33 \mathrm{GHz}}$ & $-0.69 \pm 0.07$ & $-0.59 \pm 0.08$ & & $-0.61 \pm 0.07$ & \\
\hline$\alpha_{4.7-7.2 \mathrm{GHz}}$ & $-0.61 \pm 0.04$ & $-0.55 \pm 0.07$ & & $-0.58 \pm 0.09$ & \\
\hline
\end{tabular}

Note. - For details on the calculations, see Section 2.3.1

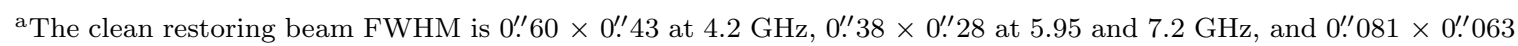
at $32.5 \mathrm{GHz}$.

Table 2.2. Best-Fit Morphology for the Nuclei of Arp 220 at $33 \mathrm{GHz}$

\begin{tabular}{|c|c|c|c|c|}
\hline \multirow{2}{*}{ Parameter } & \multicolumn{2}{|c|}{ East nucleus } & \multicolumn{2}{|c|}{ West nucleus } \\
\hline & Deconvolved $^{\mathrm{a}}$ & Convolved ${ }^{\mathrm{b}}$ & Deconvolved $^{\mathrm{a}}$ & Convolved \\
\hline \multicolumn{5}{|c|}{ Exponential Disk Model } \\
\hline Scale length (pc) & $30.3 \pm 4.6$ & $34.0 \pm 5.1$ & $21.0 \pm 3.2$ & $25.4 \pm 3.8$ \\
\hline Peak intensity $\left(\mathrm{mJy}\right.$ beam $\left.^{-1}\right)$ & $6.0 \pm 0.7$ & $4.8 \pm 0.6$ & $13.4 \pm 1.6$ & $9.0 \pm 1.1$ \\
\hline Position angle $\left(^{\circ}\right)$ & $54.7 \pm 0.6$ & $55.4 \pm 0.6$ & $79.4 \pm 0.8$ & $77.3 \pm 0.8$ \\
\hline Inclination $\left({ }^{\circ}\right)$ & $57.9 \pm 0.6$ & $55.4 \pm 0.6$ & $53.5 \pm 0.5$ & $49.1 \pm 0.5$ \\
\hline $\mathrm{R}_{50}(\mathrm{pc})^{*}$ & $50.8 \pm 7.6$ & $57.0 \pm 8.6$ & $35.2 \pm 5.3$ & $42.7 \pm 6.4$ \\
\hline \multicolumn{5}{|c|}{ Two Dimensional Gaussian Fitting } \\
\hline FWHM major axis (pc) & $85.9 \pm 8.6$ & $90.8 \pm 9.1$ & $63.7 \pm 6.4$ & $70.2 \pm 7.0$ \\
\hline FWHM minor axis (pc) & $46.3 \pm 4.6$ & $51.8 \pm 5.2$ & $38.0 \pm 3.8$ & $44.7 \pm 4.5$ \\
\hline Position angle $\left(^{\circ}\right)$ & $56.0 \pm 1.1$ & $56.5 \pm 1.1$ & $78.7 \pm 1.6$ & $77.1 \pm 1.5$ \\
\hline \multicolumn{5}{|c|}{ 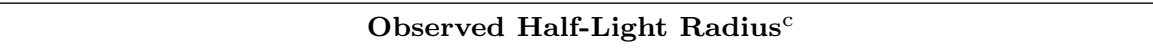 } \\
\hline $\mathrm{R}_{50 \mathrm{sky}}(\mathrm{pc})$ & & $73.3 \pm 7.5$ & & $45.5 \pm 3.7$ \\
\hline
\end{tabular}

Note. - The reported parameters were obtained by fitting a 2-D exponential and Gaussian distribution, respectively. The quoted uncertainties reflect the systematic uncertainty from varying the goodness of fit statistic or other methodology in the fit. In the case of the peak intensity, the uncertainty is determined by the flux density calibrator error, $12 \%$. In all cases, the errors from the fit are negligible. For Arp $220\left(d_{L}=77 \mathrm{Mpc}\right), 10 \mathrm{pc} \approx 0{ }^{\prime \prime} 03$ or $0{ }^{\prime \prime} 1=36.9 \mathrm{pc}$

${ }^{\text {a }}$ Parameters that construct the best image, compared to the observed one, after convolving the model with the reported clean beam (see Section 2.3.3 for details).

${ }^{\mathrm{b}}$ Best fit parameters that reconstruct the observed image without accounting for the beam.

${ }^{c}$ Taking $\mathrm{R}_{50 \text { sky }}=\sqrt{\mathrm{A}_{50 \text { sky }} /(\pi \cos \mathrm{i})}$, where $i$ is the inclination obtained from the exponential disk model and $A_{50 \text { sky }}$ is the observed area enclosing half of the total $33 \mathrm{GHz}$ flux density. The effects of the beam are not accounted for in this size metric.

*This is an analytical solution obtained by using the scale length parameter from the model. We refer to the deconvolved column as $R_{50 d}$. 
ures 2.1 and 2.2, we have also shown that the size measurement agrees with that implied by the RSN/SNR distribution (Lonsdale et al. 2006a). In fact, Herrero-Illana et al. (2012) derive scale lengths for the RSN/SNR distribution, from Lonsdale et al. (2006a) and Parra et al. (2007), that are consistent with the results shown in Table 2.2 .

The p.a.'s of the east and west nuclei agree well with those of the kinematic major axes of the disks measured from sub-millimeter CO observations (Sakamoto et al. 1999, 2008), H I absorption observations from Mundell et al. (2001), and H53 $\alpha$ radio recombination line Rodríguez-Rico et al. (2005). The velocity gradients along these position angles on individual nuclei have also been observed in the $2 \mu \mathrm{m} \mathrm{H}_{2}$ line (Genzel et al. 2001) which traces hot molecular gas and $2.3 \mu \mathrm{m} \mathrm{CO}$ absorption (the latter traces stellar velocities: (Engel et al. 2011)).

We performed two checks on the size measurements. First, as a point of comparison, we report in Table 2.2 a 2-D Gaussian fit to each nucleus at $33 \mathrm{GHz}$. We obtained deconvolved FWHM sizes of 0 ' $23 \times 00^{\prime \prime} 13\left(86 \times 46 \mathrm{pc}^{2}\right)$ for the eastern and $0{ }^{\prime \prime} 17 \times 0^{\prime \prime} 10\left(64 \times 38 \mathrm{pc}^{2}\right)$ for the western nucleus. These sizes agree fairly well with previous, marginally resolved, estimates at other frequencies. Downes \& Eckart (2007) found a deconvolved major axis size of $0 ! 19=70 \mathrm{pc}$ for the western nucleus at $1.3 \mathrm{~mm}$. Sakamoto et al. (2008) found major axis sizes of $0^{\prime \prime} 27=100 \mathrm{pc}$ (FWHM, east) and $0^{\prime \prime} 16=59 \mathrm{pc}$ (FWHM, west) at $860 \mu \mathrm{m}$. However, we show through radial profiles (Figure 2.2) that the disks are better described by an exponential morphology. In fact, the deconvolved Gaussian fit would underestimate the deconvolved half-light diameter of the disks by $9 \%$ (west) and $15 \%$ (east), if we account for inclination effect in the gaussian fit, i.e, the deconvolved $\mathrm{FWHM}_{\text {major }}$ is smaller by $\sim 9 \%$ and $15 \%$ when compared to the deconvolved half-light diameter $\left(2 \times \mathrm{R}_{50 \mathrm{~d}}\right)$.

Second, we calculated the area on the sky containing half of the flux associated with each nucleus. This very basic measure still suffers from beam dilution and inclination effects, but provides a measure of size that is independent of the functional form. We derived this image-based $A_{50 \text { sky }}$ by identifying the isointensity contour that encloses $50 \%$ of the total flux density of each nucleus. We summed the area of the pixels (pixel size $=00^{\prime \prime} 02$ ) enclosed within that contour and estimate the observed 
radius for $i=55.4^{\circ}$ (east) and $i=49.1^{\circ}$ (west), and $\mathrm{R}_{50 \text { sky }}=\sqrt{\mathrm{A}_{50 \text { sky }} /(\pi \cos \mathrm{i})}$. This is $\sim 73 \mathrm{pc}$ for the eastern nucleus and $\sim 46 \mathrm{pc}$ for the western nucleus. We report the observed $R_{50 \text { sky }}$ values in Table 2.2. As with the Gaussian, the measured area shows broad agreement with the exponential profile fitting, though differing in detail.

We created deprojected, azimuthally averaged radial profiles of $33 \mathrm{GHz}$ intensity to assess the accuracy of our models and compare the structure of the nuclei to that of the RSN/SNR number density map. Assuming a thin tilted ring geometry, we calculated deprojected profiles for the observed emission, the emission in the convolved model, and the RSN/SNR number density map. In each case, the center of the profiles correspond to the highest intensity pixel in the observed image for each nucleus. We plot these profiles in Figure 2.2. We included only emission above a signal-to-noise ratio of 3 and then averaged the intensity in a series of inclined, $\sim 00^{\prime} 035$-wide (half the clean beam size) rings, adopting the best-fit model inclination and p.a. from our modeling (Table 2.2). Note that these rings oversample the $\sim 0$ '.07 beam, so that adjacent bins in Figure 2.2 are not independent. We normalized the RSN/SNR radial profile to match the $33 \mathrm{GHz}$ profile at $r \approx 0$ '.09. The plotted error bars were calculated from the standard deviation of the flux within each annulus divided by the square root of the area in that annulus expressed in units of the beam size (i.e., the number of independent beams).

In Figure 2.2, we show that the exponential model matches the data well for both nuclei, matching slightly better for the western nucleus. Meanwhile, the Gaussian profile is not as good as the exponential profile when compared to the observed data, being particularly poorer in the outer parts of the disks. The linearity of the semilog profiles also confirms (and motivates) our adoption of an exponential functional form. The RSN/SNR radial profiles mostly follow the integrated radio emission profiles (and thus also the model). The agreement is better in the western nucleus. The eastern nucleus lacks a bright central peak and shows a somewhat more scattered distribution, which could be caused by stochasticity and timescale effects; i.e., there just may not be enough RSNe/SNRs visible to give a smooth appearance (compare to bottom right panel of Figure 2.1 to see the clumpy nature of the SNe distribution). The same idea applies for the outskirts of the western nucleus. In the rest of the paper, we 
will consider that the RSN/SNR number density distribution follow the continuum emission observed at $33 \mathrm{GHz}$ closely enough that we can take our measured $33 \mathrm{GHz}$ sizes as indicative of the distribution of active star formation in Arp 220.
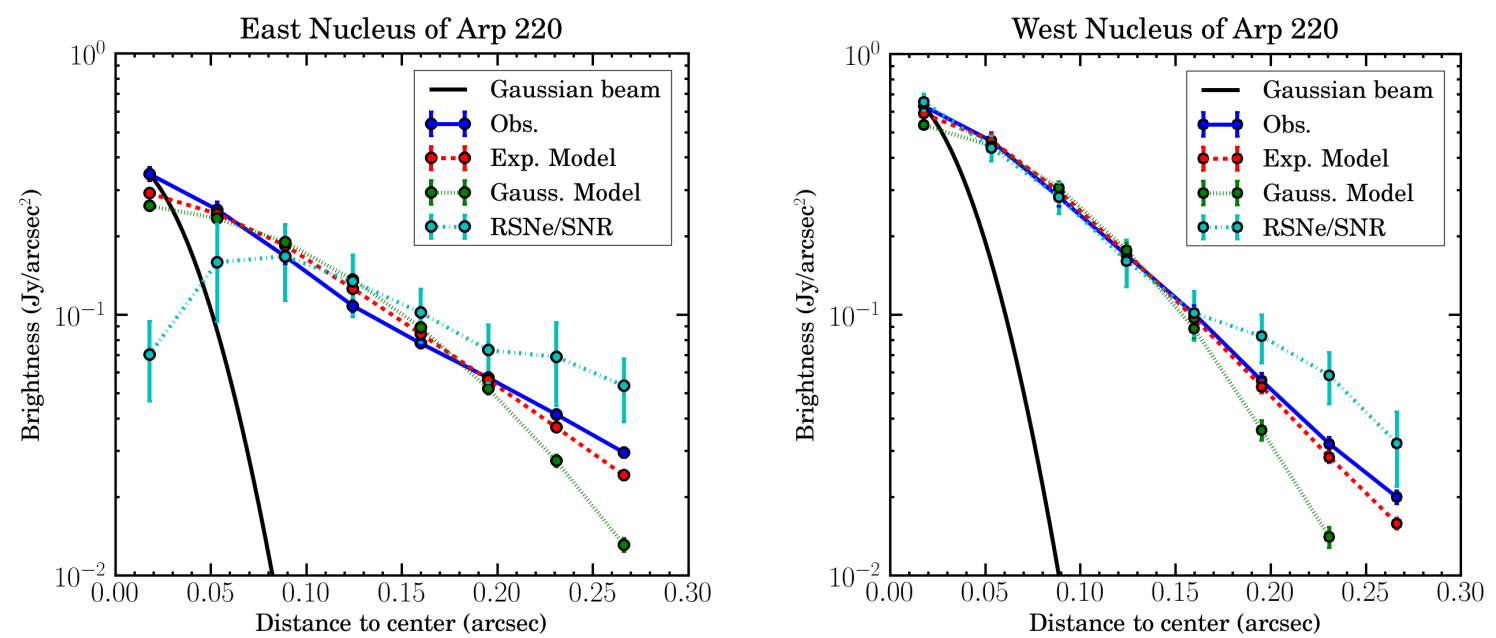

Fig. 2.2.- : Azimuthally averaged radial profiles for the deprojected image of the east nucleus (left) and west nucleus (right) in semilog space. Blue solid curves are $33 \mathrm{GHz}$ emission, and cyan dotted dashed line curves are the RSN/SNR number density profiles scaled to match the $33 \mathrm{GHz}$ radial profile at 0.'09. Red dotted lines and green dashed lines are the radial profiles of the exponential and Gaussian model images, respectively, convolved with the beam. The black solid lines represent the Gaussian beam radial profile.

\subsubsection{Brightness Temperatures}

The brightness temperature, $\mathrm{T}_{\mathrm{b}}$, can be used to constrain the emission mechanism and energy source, and may give clues regarding the optical depth. With well resolved sizes, we can circumvent beam dilution that often confuses estimates of $T_{b}$. We calculated $\mathrm{T}_{\mathrm{b}}$ using the Rayleigh-Jeans approximation via

$$
T_{b}=\left(\frac{S_{\nu}}{\Omega_{\text {source }}}\right) \frac{c^{2}}{2 k_{B} \nu^{2}}
$$

with $S_{\nu}$ the flux density at frequency $\nu$ and $\Omega_{\text {source }}$ the area subtended by the source.

We report average Rayleigh-Jeans brightness temperatures, $T_{b}$, in Table 2.3. From our model, we take the area $\mathrm{A}_{50 \mathrm{~d}} \equiv \pi \mathrm{R}_{50 \mathrm{~d}}^{2}$ that we expect to enclose half the emission if the system were viewed face on (see the "deconvolved" column in Table 2.2). 
Assuming this to be the true area of the disk at all frequencies, we derive average $T_{b}$ over the half-light region. This means we used half of the observed flux density for $\mathrm{S}_{\nu}$ and $\mathrm{A}_{50 \mathrm{~d}}$ for $\Omega_{\text {source }}$ in Equation 2.2. Our rationale for this assumption is that the $33 \mathrm{GHz}$ image appear to be optically thin, high-resolution tracer of the distribution of recent star formation. Assuming that this structure is common across wavelength regimes allows us to use a "true" size in place of a size observed with a much coarser beam. We also calculated the peak $\mathrm{T}_{\mathrm{b}}$ at each band from the peak flux density and the area of the clean beam at each frequency. This peak $\mathrm{T}_{\mathrm{b}}$ is higher at $33 \mathrm{GHz}$ than at $6 \mathrm{GHz}$; this simply reflects that the area of Arp 220 at $33 \mathrm{GHz}$ is smaller than the beam size at $6 \mathrm{GHz}$. For exactly this reason - the small size of Arp 220 and the variable resolution at different frequencies - the peak measurement has limited utility and we only report the "average" version.

The average $\mathrm{T}_{\mathrm{b}}$ is from $10^{4.4} \mathrm{~K}$ at $6 \mathrm{GHz}$ to $10^{2.5}(\approx 300) \mathrm{K}$ at $33 \mathrm{GHz}$, for the east nucleus and $10^{4.8} \mathrm{~K}$ at $6 \mathrm{GHz}$ to $10^{2.9}(\approx 800) \mathrm{K}$ at $33 \mathrm{GHz}$, for the west nucleus.

\subsection{Discussion}

Figure 2.1 shows that our observations clearly separated the nuclei at both 6 and $33 \mathrm{GHz}$ and resolve the structure of both nuclei is resolved at $33 \mathrm{GHz}$. We find a projected nuclear separation of $0{ }^{\prime \prime} 96 \pm 00^{\prime \prime} 01$ (354 \pm 4 pc), in agreement with previous works (e.g., Scoville et al. 1998; Soifer et al. 1999; Rodríguez-Rico et al. 2005; Sakamoto et al. 2008). In the following subsections, we discuss the radio continuum emission processes, the correspondence with gas and dust emission, and consequences

Table 2.3. Average Brightness Temperatures in Arp 220

\begin{tabular}{cccc}
\hline \hline $\begin{array}{c}\text { Frequency } \\
(\mathrm{GHz})\end{array}$ & $\begin{array}{c}\text { Total system } \\
\log \left[T_{b}(\mathrm{~K})\right]\end{array}$ & $\begin{array}{c}\text { East nucleus } \\
\log \left[T_{b}(\mathrm{~K})\right]\end{array}$ & $\begin{array}{c}\text { West nucleus } \\
\log \left[T_{b}(\mathrm{~K})\right]\end{array}$ \\
\hline 5.95 & $4.64 \pm 0.07$ & $4.43 \pm 0.10$ & $4.81 \pm 0.10$ \\
32.5 & $2.7 \pm 0.1$ & $2.5 \pm 0.1$ & $2.9 \pm 0.1$ \\
\hline
\end{tabular}

Note. - Integrated values are calculated within $\mathrm{A}_{50 \mathrm{~d}}$ (deconvolved modeled size) at $33 \mathrm{GHz}$ (more in Section 2.3.4). The uncertainties follow from propagation of uncertainties quoted earlier in this paper. 
of the small sizes of the emission regions. There is a large scale agreement between the locations of radio continuum emission, CO, and RSNe/SNRs, suggesting that the sizes of the radio continuum sources may be viewed as characteristic of the system.

\subsubsection{Synchrotron Produces Most of the $33 \mathrm{GHz}$ Emission}

Synchrotron radiation appears to produce most of the continuum emission at both 6 and $33 \mathrm{GHz}$. The high brightness temperature of a few $\times 10^{4} \mathrm{~K}$, inferred at $6 \mathrm{GHz}$ by using the $33 \mathrm{GHz}$ nuclear sizes, argues in this direction. This high brightness temperature cannot come from HiI regions, even if they are completely opaque, because in a purely thermal environment, the electron temperature of such regions should not exceed $10^{4} \mathrm{~K}$. If we combined the high brightness temperature with the observed internal $\mathrm{C}$ band spectral index of the total system, $\alpha_{4.7-7.2 \mathrm{GHz}}=-0.61 \pm 0.04$, we infer that most of the emission at $6 \mathrm{GHz}$ is synchrotron.

The spectral index between 6 and $33 \mathrm{GHz}, \alpha_{6-33 \mathrm{GHz}}=-0.69 \pm 0.07$, matches the internal $\mathrm{C}$ band $\alpha$ of the total system within the errors; the same is true for the two nuclei separately (see Table 2.1). The similarity between $\alpha_{4.7-7.2 \mathrm{GHz}}$ and $\alpha_{6-33 \mathrm{GHz}}$ indicates no significant spectral flattening between 6 and $33 \mathrm{GHz}$, suggesting that synchrotron dominates the emission across this range of frequencies. Reinforcing this point, our total flux density and spectral index agree with the predictions made by Anantharamaiah et al. (2000) (see their Figure 10b). They found synchrotron emission to dominate below $\sim 60 \mathrm{GHz}$, and estimated the thermal fraction at $6 \mathrm{GHz}$ and $33 \mathrm{GHz}$ to be $\sim 15 \%$ and $35 \%$, respectively. If we assume a thermal fraction of $35 \%$, a non-thermal spectral index of -0.76 (see Table 9 in Anantharamaiah et al. 2000) and a typical thermal spectra index of -0.1 , we obtain $\alpha_{6-33 \mathrm{GHz}} \approx-0.60$ and $\alpha_{4.7-7.2 \mathrm{GHz}} \approx-0.66$, which lie within $\sim 1.3 \sigma$ of the observed values.

1)We consider this to be insignificantly different to what we observe, if we take into account the uncertain nature of the predictions and the uncertainties in the observations. Then, this result is consistent with our observation of a constant spectral index from 6 to $33 \mathrm{GHz}$. Overall, our results are consistent with previous results showing lower thermal fractions at $33 \mathrm{GHz}$ for merging starbursts compared to normal galaxies 
(Murphy 2013).

2) The difference between the predicted spectral indices is 0.06 , while for the observed values is closer to $-0.08 \pm 0.11$. If we assume the predicted values are the true ones, this slight discrepancy could indicate potential opacity effects between 4.7 and 7.2 GHz (see Section 2.4.3), or the presence of a steeper non-thermal spectral index between 6 and $33 \mathrm{GHz}$. However, this discrepancy does not affect our interpretation of non thermal emission dominating at $33 \mathrm{GHz}$. In fact, a higher thermal fraction at $33 \mathrm{GHz}$ will only make this discrepancy worse (see below). Overall, our results are consistent with previous results showing lower thermal fractions at $33 \mathrm{GHz}$ for merging starbursts compared to normal galaxies (Murphy 2013).

The overall star formation rate of the system provides an alternate way to estimate the expected thermal radio continuum emission. Beginning with the IR (8 to 1000 $\mu \mathrm{m})$ luminosity of Arp 220, we estimate the expected thermal luminosity of the system if the IR is all due to star formation by following star formation rate conversions from Table 8 in Murphy et al. $(2012)^{3}$ and assuming an electron temperature of $7500 \mathrm{~K}$ (Anantharamaiah et al. 2000). This approach predicts a thermal fraction of $\sim 55 \%$ at $33 \mathrm{GHz}$ and $\sim 20 \%$ at $6 \mathrm{GHz}$. This is in good agreement with the thermal fraction at $33 \mathrm{GHz}$ obtained in Condon (1992) for a prototypical starburst, M82. However, if we derive the expected spectral index as we did at the end of the previous paragraph (assuming $55 \%$ of thermal fraction at $33 \mathrm{GHz}$ ), we obtain $\alpha_{6-33 \mathrm{GHz}} \approx-0.42$ and $\alpha_{4.7-7.2 \mathrm{GHz}} \approx-0.63$, which deviates considerably from what we observe between 6 and $33 \mathrm{GHz}$. Note that $\alpha_{4.7-7.2 \mathrm{GHz}}$ does not vary significantly from what we observe, this is due to the small thermal fraction expected at this frequency range.

The easiest explanation for the lower-than expected thermal flux is that a significant fraction of the ionizing photons produced by young stars are absorbed by dust before they produce ionizations. This would lower the free-free estimate in the calculation. We estimate that to match our observations, we would require that at least $20 \%$ of the ionizing photons be absorbed by dust. This number seems plausible

\footnotetext{
${ }^{3}$ Murphy et al. (2012) uses a Kroupa IMF to derive the theoretical SFR conversions. The operation is equivalent to using such an IMF to relate the ionizing photon production (traced by thermal radio emission) to bolometric luminosity (traced by IR).
} 
for an environment as dust embedded as Arp 220 (see Section 2.4.3) and is consistent with some of the arguments made when considering the apparent deficit of IR cooling line emission (Díaz-Santos et al. 2013, and references therein). Alternatively, an IMF that produces more bolometric light (and thus IR and likely SNe) relative to ionizing photons could resolve the discrepancy. That is, we could invoke an "intermediateheavy" IMF compared to that used in Murphy et al. (2012). We could also reconcile our two estimates if the synchrotron spectral slope decays drastically between 6 and $33 \mathrm{GHz}$, so that the apparent 33-6 GHz index is a combination of very steep, curving synchrotron and emerging thermal emission. However, this would require that thermal emission make up most of the SED at higher frequencies, which is not observed (see Anantharamaiah et al. 2000; Clemens et al. 2010).

Our best interpretation of the data is that the $33 \mathrm{GHz}$ emission is mostly synchrotron, in mild contrast with a typical starburst galaxy (see Figure 1 in Condon 1992). We suggest that the most likely cause is the suppression of thermal radio emission as dust absorbs ionizing photons.

\subsubsection{The Radio Emission Coincides with Gas, Hot Dust and RSN/SNR}

In the top-right panel of Figure 2.1, we show the $6 \mathrm{GHz}$ emission is largely co-spatial with $\mathrm{CO}$ emission. The $6 \mathrm{GHz}$ emission is our more sensitive band, with a beam nearly matched to the $\mathrm{CO}$, and — as just discussed - we expect that it traces the same synchrotron emission as the $33 \mathrm{GHz}$. The $\mathrm{CO}$ and $6 \mathrm{GHz}$ emission cover roughly the same area, have broadly coincident peaks, and both show an extended faint feature to the southwest (Mazzarella et al. 1992, note a similar coincidence between $18 \mathrm{~cm}$ emission and the Arp 220 starburst traced in the near-IR). The distributions of CO and $6 \mathrm{GHz}$ emission do significantly differ in detail. The ratio of fluxes for the two nuclei is 1:2 (east:west) for CO and almost 1:1 for continuum. In the west nucleus, the morphology is more centrally concentrated at $6 \mathrm{GHz}$ compared to the CO map. Some of these differences may reflect real differences between the current gas reservoir and recent star formation, but they may also reflect temperature and optical depth effects. 
The CO $(3 \rightarrow 2)$ emission in this region shows good evidence for optical thickness (Sakamoto et al. 2008), and the densities are high enough that the gas temperature will be likely coupled to the dust $(\sim 100 \mathrm{~K})$. Therefore, making a straightforward interpretation of the $\mathrm{CO}$ in terms of column density is challenging. We draw the broad conclusion from the top left panel of Figure 2.1 that the synchrotron originates from the same region as, and in very rough proportion to, the molecular gas supply.

A similar situation is also observed on smaller spatial scales by the comparison of the RSN/SNR number density map to the $33 \mathrm{GHz}$ map (see bottom right panel of Figure 2.1). The distributions are co-spatial, but the continuum map appears smoother than the map made from individual RSN/SNR. This is particularly evident in the eastern nucleus, where the covering fraction of VLBA point sources is small - perhaps a result of stochasticity in the rate and lifetime of SN visible using VLBI measurements. The radial profiles in Figure 2.2 highlight the quantitative agreement between the continuum and the RSNe/SNRs distribution even more. After azimuthal averaging, the VLBA point source maps are a fairly close match to the $33 \mathrm{GHz}$ continuum. This is also supported by the agreement between the scale lengths reported in Table 2.2 and those found by Herrero-Illana et al. (2012) based on the radial profiles of the RSNe/SNRs distribution observed by Lonsdale et al. (2006a) and Parra et al. (2007).

Such a close match between the $33 \mathrm{GHz}$ continuum extent and the RSN/SNR number density map may not be too surprising: if synchrotron radiation arises from cosmic ray (CR) electrons accelerated by SN shocks, then the $33 \mathrm{GHz}$ continuum emission might be expected to resemble a "puffed up" version of the RSN/SNR distribution due to the diffusion of CR electrons. Instead the distributions match quite well, consistent with most of the $33 \mathrm{GHz}$ emission coming from very close to the original RSN/SNR and little diffusion or secondary CR electron production. This lack of significant propagation could be explained by the cooling timescales being much smaller than the diffusion time. This is expected in compact starbursts with magnetic fields of the order of a mG (see measurements from Robishaw et al. 2008; McBride et al. 2014, based on Zeeman splitting of OH megamaser emission), like Arp 220 (see Figure 1 in Murphy 2009), though not in normal galaxies (Murphy et al. 
2006). For Arp 220, the cooling time of $\mathrm{CR}$ electrons at $33 \mathrm{GHz}$ is $\sim 10^{3} \mathrm{yr}$, which is a combination of synchrotron, bremsstrahlung, ionization and inverse Compton (IC) losses. We make use of Equation 7 in Murphy (2009) for CR electrons with energies greater than $1 \mathrm{GeV}$ to estimate that the synchrotron emitting electrons at $33 \mathrm{GHz}$ only have time to propagate about $5 \mathrm{pc}$, which is about $1 / 10$ th of the size that we have measured for the nuclei. This short diffusion scale yields a synchrotron image that looks very similar to the sites of original CR production (the RSN/SNR) and thus the sites of active star formation. The advantage of the VLA continuum in this case is that in exchange for coarser native resolution, we achieve sensitivity to most of the flux and spatial scales of interest (and potentially still probe a longer timescale).

The similarity of $6 \mathrm{GHz}, 33 \mathrm{GHz}$, CO surface brightness, and the RSN/SNR number density distributions lead us to view our $33 \mathrm{GHz}$ measurement as indicative of the true size of the main disks of star formation and, presumably, gas and hot dust. These morphologies are also consistent with the nuclear morphologies measured in mid-IR with the Keck telescope (Soifer et al. 1999). Perhaps surprisingly, the two disks appear fairly similar in terms of profile, scale length, and observed flux. The western nucleus appears hotter and more compact but the differences are small factors, not an order of magnitude. The physical interpretation of such similarities is unclear. Possible explanations include a similarity in the progenitors, or some "loss of memory" during the process of funneling gas to the center of the galaxies during the ongoing interaction.

\subsubsection{The Nuclear Disks are the Most Extreme Starburst Environments in the Local Universe}

\section{Gas Surface Densities}

Current best estimates of the dynamical mass per nucleus are $\sim 6 \times 10^{9} \mathrm{M}_{\odot}$ within $\sim 100$ pc of each nucleus (Engel et al. 2011). These values are still uncertain, with $\sim 2 \times 10^{9} \mathrm{M}_{\odot}$ representing a likely lower limit in both nuclei (Engel et al. 2011). The dynamical mass represents an upper limit on the gas content. Based on dynamical modeling and CO imaging, Downes \& Solomon (1998) estimated the gas content at 
$1.1 \times 10^{9}$ and $0.6 \times 10^{9} \mathrm{M}_{\odot}$ for the eastern and western nucleus, but embedded in a larger gas disk with total mass $\sim 3 \times 10^{9} \mathrm{M}_{\odot}$ (see also Sakamoto et al. 1999, 2008; Downes \& Eckart 2007). These estimates mix dynamical modeling with observations of low-J CO line (up to $\mathrm{J}=3 \rightarrow 2$ ) measurements that are likely very optically thick in Arp 220. Papadopoulos et al. (2012) provide an alternative, but unresolved, estimate by focusing on higher J CO transitions and high critical density tracers (e.g., $\mathrm{HCN})$ to estimate a total molecular gas mass of $\sim(15-28) \times 10^{9} \mathrm{M}_{\odot}$ for the entire system. The difficulty with this estimate is apportioning this gas mass to the various components of the system. We consider a conservative approach to be the following: we assume that half of the total molecular gas mass is equally distributed between the two nuclei and the other half in an outer disk (e.g., see Sakamoto et al. 1999, for evidence of an outer disk). This implies $\sim 3.5-7 \times 10^{9} \mathrm{M}_{\odot}$ of gas per nucleus from Papadopoulos et al. (2012). This remains in moderate tension with the dynamical masses because it would imply very high gas fractions, but given the mismatch in scales (the dynamical masses are estimated on $\sim 100$ pc scales) and uncertainties in modeling, a factor of $\sim 3-5$ uncertainty seems plausible (Sakamoto et al. 2008).

The areas that we measure for the Arp 220 nuclei are stunningly small, especially when compared to the integrated properties of the system. We adopt the literature gas mass of the nuclei as $3.5_{-1.5}^{+3.5} \times 10^{9} \mathrm{M}_{\odot}$, with the lower bound set by the Engel et al. (2011) values, the upper bound set by Papadopoulos et al. (2012), and the best estimate consistent (with modest tension) with the latter. We further expect half of the gas mass of each to be distributed within the half-light deconvolved, face-on, area $\left(A_{50 d}\right)$ of our radio images (these trace star formation, so we implicitly assume that gas and star formation track one another within the system). We thus compare $\sim 1.75_{-0.75}^{+1.75} \times 10^{9} \mathrm{M}_{\odot}$ to our half-light areas to estimate average, nuclear, gas surface densities. The deprojected, deconvolved gas surface densities are $2.2_{-1.0}^{+2.1} \times 10^{5} \mathrm{M}_{\odot} \mathrm{pc}^{-2}$ (east) and $4.5_{-1.9}^{+4.5} \times 10^{5}$ (west) $\mathrm{M}_{\odot} \mathrm{pc}^{-2}$. These translate to an average, nuclear, total hydrogen column densities of $2.7_{-1.2}^{+2.7} \times 10^{25} \mathrm{~cm}^{-2}$ (east) and $5.6_{-2.4}^{+5.5} \times 10^{25} \mathrm{~cm}^{-2}$ (west) (divide these numbers by 2 for $\mathrm{H}_{2}$ column densities). These nuclear hydrogen columns are $\sim 3-4$ orders of magnitude higher than those derived from X-ray observations (e.g., Clements et al. 2002; Iwasawa et al. 2005), but they roughly agree with those 
derived from observations at $860 \mu \mathrm{m}$ (Sakamoto et al. 2008) and $434 \mu \mathrm{m}$ (Wilson et al. 2014). The gas surface densities that we derive roughly resemble the maximum stellar surface density of $\sim 10^{5} \mathrm{M}_{\odot} \mathrm{pc}^{-2}$ found in a compilation of literature data by Hopkins et al. (2010). Given the large uncertainty in our mass estimate (and the scatter in the Hopkins et al. (2010) compilation) Arp 220 appears consistent with producing such a "maximal" stellar surface density system. This is especially true when one considers that feedback and further evolution of the system may reduce the efficiency (final fraction of gas converted to stars) in the nuclei below unity (a factor of $\sim 1 / 3$ would produce excellent agreement).

We do not know the thickness of the disks, but by adopting a spherical geometry we can calculate a lower limit to the $\mathrm{H}_{2}$ particle densities in the nuclei ${ }^{4}$. This is $3.8_{-1.6}^{+3.8} \times$ $10^{4} \mathrm{~cm}^{-3}$ (east) and $11_{-4.5}^{+12} \times 10^{4} \mathrm{~cm}^{-3}$ (west). For comparison, a typical Milky Way molecular cloud has a surface density $\sim 100 \mathrm{M}_{\odot} \mathrm{pc}^{-2}\left(\mathrm{~N}(\mathrm{H}) \sim 10^{22} \mathrm{~cm}^{-2}\right)$ and average particle density $\mathrm{n}_{\mathrm{H}_{2}} \sim 100 \mathrm{~cm}^{-3}$. In addition to faster free fall times, correspondingly more efficient star formation, and phenomenal opacity, potential implications of such high molecular gas densities would include the secondary production of CR electrons and confinement of CR electrons.

\section{Infrared Surface Densities and Star Formation Rates}

By following the same approach, we assume the infrared emission in Arp 220 is coincident within our measured radio distribution and explore the implications. Conventionally, the infrared luminosity surface density, $\Sigma_{\mathrm{IR}}$, is defined as the luminosity per unit area of the system. We calculate $\Sigma_{\mathrm{IR}}$ by assuming that half of the infrared luminosity (from 8-1000 $\mu \mathrm{m}$ ) is generated within the deconvolved, face-on, half-light area $\left(\mathrm{A}_{50}\right)$, i.e, we calculate an average infrared luminosity surface density, within the half-light area, via

$$
\Sigma_{\mathrm{IR}}=\left(\frac{0.5 \times L_{I R}[8-1000 \mu m]}{A_{50}}\right)=\left(\frac{L_{50}}{A_{50}}\right) .
$$

\footnotetext{
${ }^{4}$ The correction to obtain the mass inside a sphere of radius $\mathrm{R}_{50 \mathrm{~d}}$ is larger than the areal correction. For simplicity, we adopt the correction appropriate for a Gaussian, so that the mass within $\mathrm{R}_{50 \mathrm{~d}}$ is $\approx 1 / 3.4$ of the total mass.
} 
Following our measurements above, we use $\mathrm{A}_{50 \mathrm{~d}} \equiv \pi\left(\mathrm{R}_{50 \mathrm{~d} \text {,east }}^{2}+\mathrm{R}_{50 \text { d,west }}^{2}\right)$ to derive a total (face on) infrared luminosity surface density of $\Sigma_{\mathrm{IR}} \sim 6.0_{-1.5}^{+2.3} \times 10^{13} \mathrm{~L}_{\odot} \mathrm{kpc}^{-2}$ 5 . If we further assume that the ratio of the fluxes between the east and west nuclei at $33 \mathrm{GHz}(\sim 1: 1)$ holds at infrared wavelengths, then using the derived radio $\mathrm{A}_{50 \mathrm{~d}} \equiv \pi \mathrm{R}_{50 \mathrm{~d}}^{2}$ for the individual disks, we obtain $\Sigma_{\mathrm{IR}} \sim 4.2_{-0.7}^{+1.6} \times 10^{13} \mathrm{~L}_{\odot} \mathrm{kpc}^{-2}$ and $\Sigma_{\mathrm{IR}} \sim 9.7_{-2.4}^{+3.7} \times 10^{13} \mathrm{~L}_{\odot} \mathrm{kpc}^{-2}$ for the east and west nucleus, respectively. These values are more than an order of magnitude higher than those for the central $0.3 \mathrm{pc}$ of the Orion nebula complex and M $82\left(\sim 2 \times 10^{12} \mathrm{~L}_{\odot} \mathrm{kpc}^{-2}\right.$ and $\sim 9 \times 10^{11} \mathrm{~L}_{\odot} \mathrm{kpc}^{-2}$, respectively (Soifer et al. 2000)), but are closer to those found in the brightest clusters within starburst galaxies $\left(\sim 5 \times 10^{13} \mathrm{~L}_{\odot} \mathrm{kpc}^{-2}\right.$ (Meurer et al. 1997)). Our estimated surface densities are consistent with Soifer et al. (2000), who estimated infrared luminosity surface densities of $1-6 \times 10^{13} \mathrm{~L}_{\odot} \mathrm{kpc}^{-2}$ based on mid-IR Keck observations and radio data from Condon et al. (1991b).

The deprojected SFR surface density (defined as the SFR per unit area in the disk), $\Sigma_{\mathrm{SFR}}$, is a close corollary of the IR luminosity surface density. We estimate this quantity within the deconvolved, face-on, $\mathrm{A}_{50 \mathrm{~d}}$ for each nucleus at $33 \mathrm{GHz}$, using a 1:1 ratio between east and west, the radio luminosity to SFR conversion from Table 8 in Murphy et al. (2012), and an electron temperature and non-thermal spectral index of $7500 \mathrm{~K}$ and 0.76 (the spectral index in Murphy et al. (2012) is defined with the opposite sign compared to our definition), respectively, from Anantharamaiah et al. (2000). We obtain a $\Sigma_{\mathrm{SFR}}$ of $\sim 10^{3.7 \pm 0.1}$ and $10^{4.1 \pm 0.1} \mathrm{M}_{\odot} \mathrm{yr}^{-1} \mathrm{kpc}^{-2}$ within the half-light of the eastern and western nuclei, respectively (divide these numbers by two to take into account both sides of the disks). The total SFR calculated from $\mathrm{L}_{\mathrm{IR}}$, $180 \mathrm{M}_{\odot} \mathrm{yr}^{-1}$, and from the total radio flux density at $33 \mathrm{GHz}\left(\mathrm{L}_{\text {Radio }}\right), 195 \mathrm{M}_{\odot} \mathrm{yr}^{-1}$, differ by only $\sim 10 \%$, consistent with Arp 220 lying on the (33 GHz) radio-to-far infrared correlation (and meaning that we would obtain essentially the same $\Sigma_{\mathrm{SFR}}$ for either luminosity). The radio SFR value differs by $20 \%$ from that derived from Anantharamaiah et al. (2000), which we consider to be within the uncertainties of such calculations.

\footnotetext{
${ }^{5}$ The uncertainties in this value, and in the rest of this section, correspond to the errors associated to $\mathrm{R}_{50 \mathrm{~d}}$.
} 
$\Sigma_{\text {IR }}$ tells us coarsely about the density of IR luminosity per unit area, but not necessarily the flux at the surface of the source, which may have important implications for feedback and depends on the detailed geometry of the system. A spherical geometry provides a useful limit on the flux at the surface of the system. In this case, $\mathrm{F}_{\text {sphere }}=\mathrm{L}_{50} /\left(4 \pi \mathrm{R}_{50 \mathrm{~d}}^{2}\right)$ will be the flux at the surface of a sphere of radius $R_{50 d}$ with the luminosity of Arp 220. Using the measured radio sizes, $\mathrm{F}_{\text {sphere }} \sim 1.5_{-0.4}^{+0.6} \times 10^{13} \mathrm{~L}_{\odot} \mathrm{kpc}^{-2}$ for the entire system, $\sim 1.1_{-0.2}^{+0.4} \times 10^{13} \mathrm{~L}_{\odot} \mathrm{kpc}^{-2}$ for the eastern nucleus and $\sim 2.4_{-0.6}^{+0.9} \times 10^{13} \mathrm{~L}_{\odot} \mathrm{kpc}^{-2}$ for the western nucleus. A less extreme case, one that may well apply to Arp 220, is a two-sided disk. With one half of the luminosity emergent from each side, we have $\mathrm{F}_{\text {disk }}=\mathrm{L}_{50} /\left(2 \pi \mathrm{R}_{50 \mathrm{~d}}^{2}\right)$, twice the spherical case. As one would expect, these values are lower than the simple $\Sigma_{\mathrm{IR}}$, but they also differ from one another, reinforcing the importance of geometry to the physics of the source ${ }^{6}$.

Yet another subtlety arises in the specific case where one wishes to calculate the flux through an area very close to, but just above one side of a disk. This quantity is relevant to the often-discussed case of radiation pressure on dust (see Section 2.4.3) but because of projection effects it is not identical to any of the above quantities. In 2.A, we show that this one-sided flux perpendicular to the disk, which we call $\mathrm{F}_{\text {near }}$ is equal to $\mathrm{L}_{\mathrm{IR}} /\left(8 \pi \mathrm{R}_{50 \mathrm{~d}}^{2}\right)$ (Equation 2.10) in general. This value is further divided by an extra factor of two for the case of the two nuclei of Arp 220 (because $L_{\mathrm{IR}}$ combines the light from the two nuclei). From this calculation, we obtain $\mathrm{F}_{\text {near }} \sim 1.5_{-0.4}^{+0.6} \times 10^{13} \mathrm{~L}_{\odot} \mathrm{kpc}^{-2}$ for the entire system (the same as for $\mathrm{F}_{\text {sphere }}$, though not for the same reason), $\sim 1.1_{-0.2}^{+0.4} \times 10^{13} \mathrm{~L}_{\odot} \mathrm{kpc}^{-2}$ for the east nucleus and $\sim 2.4_{-0.6}^{+1.0} \times 10^{13} \mathrm{~L}_{\odot} \mathrm{kpc}^{-2}$ for the west nucleus. As discussed in 2.A, these should be the most appropriate fluxes to consider when assessing the impact of pressure from radiation perpendicular to the disk.

The ratio of the flux densities between the east and west nuclei varies with frequency. Some other ratios for the east:west relation found in the literature include

\footnotetext{
${ }^{6}$ Optical depth will provide an additional complication. Here we consider the IR surface brightness near the ultimate source of the luminosity in the region of active star formation. As the radiation scatters out of the system, the geometry may change, so that the geometry of the photosphere could differ from the central source considered here.
} 
1:4 at mid-IR (Soifer et al. 1999), 1:3 at $18 \mathrm{~cm}$ (if we consider only the contribution of the point sources from Lonsdale et al. 2006a) and 1:2 at sub-mm wavelengths (Sakamoto et al. 2008). However, most of these observations do not offer high enough spatial resolution to truly isolate the contribution of each nucleus. If we ignore this issue and we assume a ratio of 1:4, and we use Equation 2.10, we obtain $\mathrm{F}_{\text {near,east }} \sim 4.5_{-1.1}^{+1.7} \times 10^{12} \mathrm{~L}_{\odot} \mathrm{kpc}^{-2}$ and $\mathrm{F}_{\text {near,west }} \sim 3.7_{-0.9}^{+1.4} \times 10^{13} \mathrm{~L}_{\odot} \mathrm{kpc}^{-2}$. In

every case, we obtain $\mathrm{F}_{\text {near }} \gtrsim 10^{13} \mathrm{~L}_{\odot} \mathrm{kpc}^{-2}$ for the west nucleus, which is always the higher intensity nucleus.

Note that the same geometric issues discussed here raise a caveat regarding the luminosity of the source, which was derived under the assumption of isotropic emission (Sanders et al. 2003; Sanders \& Mirabel 1996). An optically thick thin disk is not an isotropic emitter. However, neither do our observations constrain the geometry of the infrared photosphere, which is the relevant surface for this calculation. We discuss this issue in 2.A. Lacking information, we have assumed the isotropic luminosity throughout this paper, but note the uncertainty (see also Downes \& Eckart 2007; Wilson et al. 2014). Note that high angular resolution infrared (from 8-1000 $\mu \mathrm{m}$ ) observations are needed in order to better constrain the true morphology of the IR photosphere of Arp 220.

\section{Radiation Pressure and Maximal Starburst Models}

Scoville (2003) and Thompson et al. (2005), argued that for optically thick, dense starburst galaxies, the critical feedback mechanism acting against gravitational collapse, and thus star formation, could be radiation pressure on dust. Although there is ongoing debate about whether or not radiation pressure on dust represents the dominant feedback mechanism in compact starbursts (see Krumholz \& Thompson 2013; Socrates \& Sironi 2013; Davis et al. 2014, for further discussion), the maximal starburst model of Scoville (2003) and Thompson et al. (2005) represents an interesting point of comparison for our present work ${ }^{7}$. In Figure 2.3, which closely follows Figure 4 of Thompson et al. (2005), we present our new measurements for the flux near the

\footnotetext{
${ }^{7}$ In addition to radiation pressure, cosmic ray pressure has been forwarded as a potentially important feedback mechanism in compact starbursts (Socrates et al. 2008).
} 
surface of the source (assuming a thin disk geometry, see Section 2.4.3 and 2.A) in the context of literature observations and predictions by Thompson et al. (2005). The literature observations show a sample of ULIRGs with sizes based on $8.44 \mathrm{GHz}$ radio maps by Condon et al. (1991b) and luminosities from IRAS. Following the approach presented in 2.A, we calculate $\mathrm{F}_{\text {near }}$ following Equation 2.10 assuming half of the total infrared luminosity to be enclosed within $\mathrm{A}_{50}=\pi \mathrm{R}_{50}^{2}$, where $\mathrm{R}_{50}=b_{\text {maj }} / 2$. We used the deconvolved FWHM major axis, $b_{\text {maj }}$, from Condon et al. (1991b) in order to account for inclination effects (we only include resolved sources). Following the discussion in the previous section, the fluxes for the points in Figure 2.3 differs by a factor of 8 compared to those in Thompson et al. $(2005)^{8}$. for reasons discussed in 2.A.

The error bars of our Arp 220 values (filled points in Figure 2.3) correspond to a combination of the uncertainties in $R_{50 \mathrm{~d}}$, and uncertainty in the contribution of each nucleus to the IR luminosity, with the lower/upper limit assuming a ratio of 1:4 between east and west (Soifer et al. 1999). To be conservative, we also assume a $20 \%$ uncertainty in the assumption that half of the total IR luminosity is coming from $A_{50}$. The plotted values for the flux correspond to $\mathrm{F}_{\text {near,east }} \sim 1.1_{-0.8}^{+0.4} \times 10^{13} \mathrm{~L}_{\odot} \mathrm{kpc}^{-2}$ and $\mathrm{F}_{\text {near,west }} \sim 2.4_{-0.9}^{+2.7} \times 10^{13} \mathrm{~L}_{\odot} \mathrm{kpc}^{-2}$. In this Figure, we show the "Eddington" values for radiation pressure on dust as solid and dashed lines. These represent an envelope for which radiation pressure on dust balances self-gravity. As a result, no equilibrium star-forming system is expected to exist above this line, hence the "Eddington limit" analogy.

The precise value of the limit depends on the size, gas fraction $\left(f_{\mathrm{g}}\right)$ stellar velocity dispersion $(\sigma)$, Rosseland mean opacity $(\kappa)$, and dust-to-gas ratio of the system, leading to the large spread in the model lines seen in the Figure. Our measurements of Arp 220 appear as solid points in Figure 2.3. There, the west nucleus of Arp 220

\footnotetext{
${ }^{8}$ This factor of 8 increases for systems having more than one component, in which case we also divide the total flux among the components. For example, in the case of one individual region in Arp 299, NGC 3690, the difference is an additional factor of $\sim 4$ that comes from the contribution of that region to the total infrared luminosity of the system (Alonso-Herrero et al. 2000). For the other systems having more than one component, we used the relative contribution of each component to the integrated flux density observed at $8.44 \mathrm{GHz}$ as a template for the relative contribution at infrared wavelengths.
} 
appears among the highest brightness systems. However, the west nucleus does not clearly stand out from the other ULIRGs with respect to this value, partially because the more compact size means that the "maximal" value is larger for Arp 220 than for larger systems. Overall, all the systems plotted in Figure 2.3 lie roughly around the Eddington limit for a $\mathrm{f}_{\mathrm{g}}=0.1$ and $\sigma=200 \mathrm{~km} \mathrm{~s}^{-1}$ disk in Thompson et al. (2005), which is indicated by the blue solid line.

If we adopt $\mathrm{f}_{\mathrm{g}}=1$ and $\sigma \approx 200 \mathrm{~km} \mathrm{~s}^{-1}$ (e.g., Genzel et al. 2001), indicated by the green line in Figure 2.3, then we calculate a conservative Eddington limit of $\sim 9 \times 10^{13} \mathrm{~L}_{\odot} \mathrm{kpc}^{-2}$ for the west nucleus and $\sim 7 \times 10^{13} \mathrm{~L}_{\odot} \mathrm{kpc}^{-2}$ for the east nucleus. Refined measurements of the geometry, gas fraction, opacity, dust-to-gas ratio and kinematics are needed to specify the models more precisely. For most plausible assumed disk properties, we can say that both Arp 220 nuclei lie well below the Thompson et al. (2005) Eddington-limited starburst value, with the western nucleus being the brightest system among the local ULIRGs. If improved measurements demonstrate one or both nuclei to lie significantly above this value, one would need to consider luminosity sources other than star formation (presumably an AGN Iwasawa et al. 2005; Downes \& Eckart 2007; Rangwala et al. 2011) or question the basic assumptions about geometry and equilibrium embedded in the model, but at present little such tension appears to exist.

Another way to assess the role of radiation pressure in Arp 220 is to assume that radiation pressure does represent the dominant force acting against gravity (see 2.B) and to calculate the required gas opacity, $\kappa$, of the system in order to be in hydrostatic equilibrium. By following Equation 2.12, and using the derived values for the gas surface density and the flux for each nucleus (see Section 2.4.3 and 2.4.3), we find that $\kappa_{\text {east }} \approx 300 \mathrm{~cm}^{2} / \mathrm{g}$ and $\kappa_{\text {west }} \approx 80 \mathrm{~cm}^{2} / \mathrm{g}$ would be required for radiation pressure to balance gravity. Compared to the models from Semenov et al. (2003), which were used in the models from Thompson et al. (2005), these appear to be unrealistically high values for the gas opacity. We interpret these high values as a reinforcement of our previous findings that the nuclei of Arp 220 lie below the dusty Eddington limit for $\mathrm{f}_{\mathrm{g}} \sim 1$ and $\sigma=200 \mathrm{~km} \mathrm{~s}^{-1}$ by a factor of $\sim 10$, and then are not radiation pressure supported. 


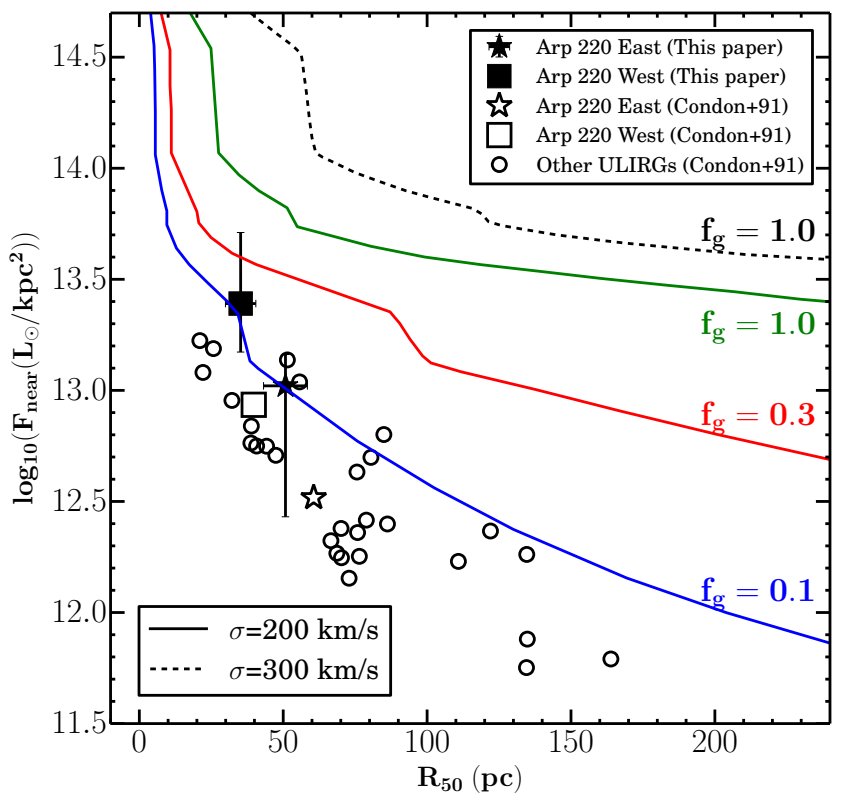

Fig. 2.3.- : Flux versus radius for local ULIRGs from Condon et al. (1991b) (open symbols), and including the values for the eastern (star symbol) and western (square symbol) nuclei of Arp 220 from this paper (filled symbols). In this figure, we represent a new version of Figure 4 of Thompson et al. (2005). The y-axis corresponds to the flux passing through a surface area near the source, $\mathrm{F}_{\text {near }}$ (see 2.A), and the x-axis to the deconvolved half-light radius. The solid lines represent the Eddington limits for several different gas fractions, $\mathrm{f}_{\mathrm{g}}$, assuming stellar velocity dispersion of $\sigma=200 \mathrm{~km} \mathrm{~s}^{-1}$. The dashed line shows the model for $\mathrm{f}_{\mathrm{g}}=1$ and $\sigma=300 \mathrm{~km} \mathrm{~s}^{-1}$. From this figure, we observe that Arp 220 appears among the brightest systems but still well below a conservative dusty Eddington limit described by the green line.

\section{Optical Depth}

The small sizes of the Arp 220 nuclei also imply that optical depth effects will be important across the spectrum, even at wavelengths as long as sub-mm. By following the same approach described in Section 2.3.4, we calculate the average Rayleigh-Jeans brightness temperatures that would be implied by combining the deconvolved halflight sizes of the nuclei with half of the $860 \mu \mathrm{m}$ continuum flux densities reported by Sakamoto et al. (2008) from lower resolution observations. At this wavelength, we find implied average brightness temperatures of $19 \mathrm{~K}$ (east) and $76 \mathrm{~K}$ (west), within the deconvolved half-light area calculated in this paper $\left(A_{50 d}\right)$, without any accounting for optical depth (which could change the size of the apparent emission). In the west nucleus, this approaches the apparent dust temperature of $\sim 90 \mathrm{~K}$ (see GonzálezAlfonso et al. 2012, for a more thorough idea of the dust temperature in Arp 220), so that optical depth must become important by this wavelength regime, with $\tau \gtrsim 1$ 
by $860 \mu \mathrm{m}$ in the western nucleus. This neglects any more complicated geometric considerations (e.g., see the discussion of an inclined geometry in Downes \& Eckart 2007), which are likely to make the situation even more confused. Optical depth effects appear less severe by $\lambda \sim 1 \mathrm{~mm}$, combining $A_{50 \mathrm{~d}}$ with half of the western nucleus dust emission from Downes \& Eckart (2007), implies an average $\mathrm{T}_{\mathrm{b}}^{1 \mathrm{~mm}} \sim$ $45 \mathrm{~K}$ within the half-light area. ${ }^{9}$ This modest mm optical depth is consistent with measurement of a spectral index steeper than $\alpha=2$ by Sakamoto et al. (2008), implying somewhat optically thin emission.

Even this simple calculation demonstrates that by sub-mm wavelengths optical depth effects cannot be neglected, especially in the western nucleus. As a result, we would expect high resolution but higher frequency observations, e.g., at sub-mm wavelengths with ALMA, to observe a moderately optically thick "photosphere" around the galaxy and so recover a larger size than we find here (e.g., see the continuum observations by Wilson et al. 2014). Similarly, line observations at these wavelengths will need to consider the effects of a moderately optically thick sub-mm continuum in their interpretation.

Further, we can estimate the optical depth at $33 \mathrm{GHz}$. The brightness temperature calculated for the entire source at $33 \mathrm{GHz}$ is $\sim 500 \mathrm{~K}$ (see Table 2.3). The thermal fraction at this frequency is $\sim 35 \%$, so that $\mathrm{T}_{\mathrm{b}}$ of the thermal emission is $\sim 175 \mathrm{~K}$. The thermal electron temperature $\left(\mathrm{T}_{\mathrm{e}}\right)$ cannot exceed $\approx 10^{4} \mathrm{~K}$ because line cooling is high at such high temperatures. Then taking $\mathrm{T}_{\mathrm{e}} \sim 10^{4} \mathrm{~K}$ and a measured $\sim 175 \mathrm{~K}$ brightness, we estimate the average $33 \mathrm{GHz}$ free-free opacity within the half-light radius to be $\tau_{\text {Thermal }} \sim \mathrm{T}_{\mathrm{b}} / \mathrm{T}_{\mathrm{e}} \sim 0.018$. Given that $\tau_{\text {Thermal }} \propto \nu^{-2.1}$, we can calculate that $\tau_{\text {Thermal }} \sim 1$ at $\nu \sim 5 \mathrm{GHz}^{10}$. At $18 \mathrm{~cm}, \tau_{\text {Thermal }}>9$, which might help explain the low VLBA flux density from Lonsdale et al. (2006a). In addition, as Sakamoto et al. (2008) note, the dust opacity is close to unity at $860 \mu \mathrm{m}$. Thus, the Arp 220

\footnotetext{
${ }^{9}$ This temperature and the $76 \mathrm{~K}$ derived from Sakamoto et al. (2008) change to $120 \mathrm{~K}$ and $215 \mathrm{~K}$, respectively, if we follow the approach of Downes \& Eckart (2007), which uses the full luminosity and defines the size of the source $\Omega_{\text {source }}$ as $\frac{\pi \theta_{\text {source }}}{4 \ln (2)}$ with $\theta_{\text {source }}$ the geometric mean between the deconvolved FWHM of the major and minor axis of the west nucleus. That is, the brightness temperature is higher without accounting for inclination effects.

${ }^{10} \tau_{\text {Thermal }}$ increases to 0.028 and $\nu$ to $6 \mathrm{GHz}$ if instead $55 \%$ of the $33 \mathrm{GHz}$ emission is thermal (see discussion in Section 2.4.1).
} 
nuclei may be transparent only near the middle of the frequency range $5 \mathrm{GHz}$ to 350 GHz. A case can thus be made that the $33 \mathrm{GHz}$ image presented here is the only existing image that is both optically thin and resolves the nuclei.

\subsubsection{Evidence at Radio Wavelengths of a Dominant AGN in the Western Nucleus}

In our observations, the western nucleus is more compact with a higher $\mathrm{T}_{\mathrm{b}}$ than the eastern nucleus. However, consistent with previous VLBI observations, we observe no significant central excess in either Arp 220 images or radial profiles (Figures 2.1 and 2.2). Parra et al. (2007) discuss the possibility that one of three VLBI point sources showing a flat spectrum $(\alpha>-0.5)$, could be an AGN. However, that is one of several possibilities that could explain the shape of their spectrum.

Most of the $33 \mathrm{GHz}$ emission that we observe comes from the compact, but still resolved, disks around the nuclei. Specifically, the nuclear beam of the west nucleus contains $20 \%$ of the total flux of the nucleus, while the other $80 \%$ arises from the more extended star forming regions (Table 2.1). Our measured $R_{50 d}$ contain similar information (see Table 2.2). We cannot rule out an AGN in the western nucleus, but if one is present it does not make a dominant, point-like contribution to the overall $33 \mathrm{GHz}$ emission on scales of $\approx 30$ pc. Similarly, Arp 220 does not exceed the "Eddington" value that might eliminate star formation as a viable power source (see Section 2.4.3). Smith et al. (1998) show that the SN rate and luminosity of Arp 220 are broadly consistent with emission only generated by star formation, though uncertainty in the IMF, SN rate, and SFR certainly would still allow an AGN contribution.

No high brightness temperature radio core indicative of an AGN is present. However, given that most AGN are radio-quiet and have weak core emission (e.g., Kellermann et al. 1989; Blundell \& Beasley 1998), the absence of a radio core does not rule out the presence of an AGN. Indeed, several studies at other wavelengths have presented evidence of a possible AGN in Arp 220 (e.g., Iwasawa et al. 2005; Downes \& Eckart 2007; Rangwala et al. 2011; Imanishi \& Saito 2014; Wilson et al. 2014), but to date there is no clear evidence that the putative AGN makes a significant 
contribution to the bolometric luminosity. If our estimate of $\mathrm{N}(\mathrm{H}) \sim 10^{25} \mathrm{~cm}^{-2}$ in Section 2.4.3 is correct, it would explain why evidence for AGN in Arp 220 has been so elusive. With such high column densities any AGN would be Compton thick and undetectable by standard AGN diagnostic tools.

\subsection{Summary}

We present new, high resolution VLA observations of the nearest ULIRG, Arp 220. Our $33 \mathrm{GHz}$ observations measure the light distribution, which originates mostly from synchrotron emission, at a wavelength where optical depth effects are likely negligible. We find exponential profiles with half-light radii of 51 and $35 \mathrm{pc}$ for the eastern and western nucleus, respectively. The distribution of $33 \mathrm{GHz}$ radio emission matches the number density distribution of recent RSNe/SNRs very well. This similarity may result from strong $(\sim \mathrm{mG})$ magnetic fields, which could yield cooling timescales for cosmic ray electrons that are short compared to the diffusion timescale. Adopting the measured $33 \mathrm{GHz}$ sizes as characteristic of the star-forming disks, we derive implied surface densities, $\mathrm{H}$ column densities and volumetric gas densities that strikingly illustrate the extreme nature of the environment present in Arp 220. Combining our size measurements with unresolved infrared measurements, we estimate total fluxes that, although very large, lie well below the conservative predicted values for the Eddington-limited "maximal starburst", though this result is sensitive to our assumptions. Regardless, the implied luminosity surface brightness for the west nucleus of Arp 220 is among the most extreme for any measured system. Given the general uncertain evidence to date of a dominant AGN in Arp 220, we conclude that the compact size and disk-like morphology clearly make Arp 220 a prototypical example of the most extreme class of star-forming systems in the local universe. 


\section{Acknowledgments}

We thank the anonymous referee for helpful comments that made this paper stronger. We thank Guillermo Damke for his helpful input in the modeling process, Norman Murray for helpful discussions at an early stage of the project, Phil Arras for useful suggestions on 2.A and Shane Davis for his input, especially in the development of 2.B, regarding radiation pressure and the Eddington limit. We thank the NRAO/UVa star formation group (especially Crystal Brogan and Kelsey Johnson) for repeated technical and scientific feedback. We also thank Nick Scoville for detailed discussions of the astrometry of Arp 220.A.S.E., G.C.P. and L.B-M. were supported by NSF grant AST 1109475. L.B-M. was also supported by Fulbright and Becas Chile - CONICYT. This research made use of the NASA/IPAC Extragalactic Database (NED), which is operated by the Jet Propulsion Laboratory, California Institute of Technology, under contract with the National Aeronautics and Space Administration, and NASA's Astrophysics Data System Bibliographic Services. The National Radio Astronomy Observatory is a facility of the National Science Foundation operated under cooperative agreement by Associated Universities, Inc. 
Appendix 


\section{A Flux Through An Area Just Above an Ex- tended Thin Disk}

Consider a geometrically thin disk with radius $R$ viewed face on. Then consider a small area parallel to the disk and at distance $d$ above the disk center. The flux, $F$, passing through the area will be

$$
F=\int I \cos (\theta) d \Omega
$$

where $d \Omega=d \phi \sin \theta d \theta$ is the area subtended by an infinitesimal part of the disk. The factor $\cos \theta$ accounts for the orientation of the area relative to the patch of emitting disk under consideration with $\cos \theta=\frac{d}{\sqrt{R^{2}+d^{2}}}$ just as $\sin \theta=\frac{R}{\sqrt{R^{2}+d^{2}}}$. I is the specific intensity, which for the optically thick case, is just the source function of the disk and is the same for all lines of sight (as the disk fills the beam). In the scenario where radiation pressure is important, we consider that near the disk high optical depth is likely and proceed in the case of Arp $220^{11}$. The integral in Equation 2.4 goes from 0 to $2 \pi$ in $\phi$ and 0 to $\sin ^{-1}\left(\frac{R}{\sqrt{R^{2}+d^{2}}}\right)$ in $\theta$. We will immediately change variables so that $x \equiv \sin \theta$ and $d x \equiv \cos \theta d \theta$. Thus,

$$
F=I \int_{0}^{2 \pi} d \phi \int_{0}^{\frac{R}{\sqrt{R^{2}+d^{2}}}} x d x=\pi I \frac{R^{2}}{R^{2}+d^{2}} .
$$

Consider the limit where $d<<R$, i.e., where the area the flux is passing through lies just above the disk. Then

$$
F_{\text {near }}=\pi I \text {, }
$$

similar to the well known relation that the flux at the surface of a blackbody is $\pi B_{\nu}^{12}$. Similarly, at large $d>>R$, as for an astronomical observation:

\footnotetext{
${ }^{11}$ In the optically thin case, $I$ will depend on the path length through the disk, which is larger by a factor of $\cos \theta$ at high viewing angles. This factor cancels with the directional $\cos \theta$ in Equation 2.4 so that the optically thin case yields a different answer.

${ }^{12} \mathrm{~F}_{\text {near }}$ will be different by a factor of two in the optically thin case.
} 


$$
F_{\text {far }}=\pi I \frac{R^{2}}{d^{2}}
$$

which is nothing more than the integral of the intensity over the solid angle subtended by the disk. The utility in this calculation is to relate $I$ back to the luminosity, which for an isotropic emitter is just $\mathrm{L}=4 \pi \mathrm{d}^{2} \mathrm{~F}_{\text {far }}$ (see the last paragraph of this appendix for some caveats regarding this assumption). Then

$$
F_{\text {far }}=\pi I \frac{R^{2}}{d^{2}}=\frac{L}{4 \pi d^{2}}
$$

so that,

$$
I=\frac{L}{4 \pi^{2} R^{2}} \text { and } \quad F_{\text {near }}=\frac{L}{4 \pi R^{2}}
$$

This $F_{\text {near }}$ is the flux through a surface near the disk and the departure from the perhaps expected $\mathrm{L} /\left(2 \pi \mathrm{R}^{2}\right)$ is that we have included the $\cos \theta$ term in the original setup to account for the projection of the incident intensity onto the unit area.

When considering real observations cast in terms of the half-light area, $A_{50}$ (and recall that the commonly used size at FWHM for a two dimensional Gaussian is $A_{50}$ ) an additional factor enters from the fact that $L_{50}=0.5 L$ for $R_{50}$. Then in general:

$$
F_{\text {near }}=\frac{L}{8 \pi R_{50}^{2}},
$$

is the flux that should be used for considering radiation pressure near a large disk (where large is defined so that $d<<R$ can hold). In Arp 220, an additional factor of two comes into play if we assume the luminosity is split between the two nuclei with a ratio of $1: 1$. This decreases the relation to $\mathrm{F}_{\text {near }}=\mathrm{L} /\left(16 \pi \mathrm{R}_{50}^{2}\right)$ for this specific case. Note the stark difference, even for the general case, from the commonly adopted $\Sigma_{\mathrm{IR}}=\mathrm{L} / \pi \mathrm{R}_{50}^{2}$.

In the case of an optically thick disk, the assumption of isotropy is not valid and $\mathrm{L} \neq 4 \pi \mathrm{d}^{2} \mathrm{~F}_{\text {far }}$, instead $\mathrm{L}=2 \pi \mathrm{d}^{2} \mathrm{~F}_{\text {far }}$. Further, the emission is not isotropically distributed, so that if the disk is inclined by an angle, $i$, with respect to the line of sight, where $i=0$ is a face-on disk, then $\mathrm{L}=2 \pi \mathrm{d}^{2} \mathrm{~F}_{\text {far }} / \cos i$. In other words, 
the emission comes only from the two sides of the disks and an observer finds more flux when the disk is viewed face on (because the constant intensity surface subtends more solid angle). This is a substantial uncertainty for Arp 220 (e.g., see Downes \& Eckart 2007): it appears to host two inclined disks and shows good evidence for optical depth at IR wavelengths. However, we are hesitant to impose any correction to the luminosity in the main analysis because we we do not know the true geometry of the infrared photosphere, which might very plausibly be more spherical and emit more isotropically than the nuclear disks picked out by our $33 \mathrm{GHz}$ observations. Therefore throughout the main text we have used the conventional $L=4 \pi d^{2} F_{\text {far }}$ but we note this substantial uncertainty.

\section{B Vertical Hydrostatic Equilibrium for a Simple Radiation Pressure Dominated Disk}

As a simple check on the plausibility of radiation pressure representing the main means of support, we consider vertical hydrostatic equilibrium in a simple gas disk. We consider an infinite slab of surface density $\Sigma$, so that the integrated weight of the column of gas at the midplane is $\pi G \Sigma^{2}$ (here $G$ is the gravitational constant). To a coarse approximation, radiation pressure can counteract this weight with a pressure set by the momentum flux of photons, $F / 2 c$ (where $c$ is the speed of light), multiplied by the total opacity of the gas column, $\kappa \Sigma$, where $\kappa$ is the cross section per unit gas mass. Then:

$$
\pi G \Sigma^{2} \sim \frac{\kappa \Sigma F}{2 c}
$$

We can then solve for $\kappa$ in terms of the other properties of the disk and the resulting $\kappa$ expresses the required effective opacity in order for radiation pressure to balance the weight of the disk:

$$
\kappa \sim \frac{2 \pi c G \Sigma}{F}
$$


If $F$ and $\Sigma$ are known, comparison of the require $\kappa$ to realistic values represents a zeroth order check of whether radiation pressure represents a viable support mechanism for a system. As a close corollary, if $\kappa$ is known or can be estimated, assessing the degree to which Equation 2.11 represents an inequality offers diagnostic of the importance of radiation pressure to the system. Note that $\kappa$, as we have written it, will depend on the dust-to-gas ratio, grain properties, overall opacity, and temperature distribution. In our simplified thin disk geometry, it does not depend directly on the size of the system but all of these properties may vary substantially as a function of disk structure. As a first order approximation, we assume the Semenov et al. (2003) model values serve as a template of typical values that would describe $\kappa$ for the nuclei in Arp 220, but to our knowledge a thorough exploration of $\kappa$ appropriate for the nuclear disks of merging galaxies remains lacking in the literature. 
Chapter 3

A $33 \mathrm{GHz}$ Survey of Local Major

Mergers: Estimating the Size of

the Energetically Dominant Region

from High Resolution

Measurements of the Radio

Continuum 


\begin{abstract}
We present new, high-resolution Very Large Array (VLA) observations of the 33 $\mathrm{GHz}$ radio continuum emission from 22 local ultraluminous and luminous infrared galaxies (U/LIRGs). These observations have spatial (angular) resolutions of 30$720 \mathrm{pc}\left(0^{\prime \prime} 07-0{ }^{\prime} \cdot 67\right)$ in an optically thin part of the spectrum, allowing for accurate measurements of the size of the energetically dominant region in these dusty, compact systems. The half-light radius implied by the $33 \mathrm{GHz}$ emission ranges from $20 \mathrm{pc}$ to $1.7 \mathrm{kpc}$ across our sample. Several arguments suggest that the emission is a roughly equal mixture of free-free and synchrotron, though the precise balance varies and remains uncertain. Both types of emission are generated by star formation and the $33 \mathrm{GHz}$ flux correlates well with the infrared emission, so we take these sizes as indicative of the region that dominates the emission energetics. This allows us to combine our sizes with unresolved flux densities at other wavelengths, accounting for the proper aperture corrections, to estimate physical conditions in these compact, dusty systems. We estimate the infrared luminosity and star formation rate per area and the molecular gas surface and volume densities. These span a wide range ( $\sim 4$ dex) among our targets, and include among the highest values measured for any galaxies. We note several implications of these calculations: At least 10 sources appear Compton thick $\left(\mathrm{N}_{\mathrm{H}} \geq 10^{24} \mathrm{~cm}^{-2}\right)$. The SFR and molecular gas surface densities in our sample combined with that of normal disk galaxies yield a nonlinear star formationmolecular gas scaling relation, though the result remains sensitive to the adopted CO-to- $\mathrm{H}_{2}$ conversion factor. Our targets have high infrared surface brightness, but 19 of the 22 sources are non radiation pressure-limited "maximal" starbursts. We also show that targets with higher surface brightness exhibit stronger [CII] deficits, consistent with the suggestion that high energy densities drive this phenomenon.
\end{abstract}

\title{
3.1 Introduction
}

Luminous and ultraluminous infrared galaxies (LIRGs: $10^{11} \leq L_{I R}[8-1000 \mu \mathrm{m}]<$ $10^{12}$, ULIRGs: $L_{I R} \geq 10^{12}$ ) host some of the most extreme environments in the local 
universe. Local U/LIRGs are primarily triggered by galaxy interactions and mergers (e.g., Sanders \& Mirabel 1996, and references therein); during this process, large amounts of gas are funneled into the central few kpc where prodigious star formation and/or AGN activity are fueled. The energetic regions within U/LIRGs are heavily embedded in dust and gas, which gives rise to their high infrared luminosities.

Their enormous gas surface densities, gas volume densities, energy densities, and high star formation rates (SFRs; up to a few times $100 \mathrm{M}_{\odot} \mathrm{yr}^{-1}$, e.g., Solomon et al. 1997; Downes \& Solomon 1998; Evans et al. 2002) make the local U/LIRGs crucial laboratories to understand the physics of star formation and feedback. Indeed, these systems have one of the highest SFR and gas surface densities measured for any system (e.g., Downes \& Solomon 1998; Daddi et al. 2010; Liu et al. 2015; Lutz et al. 2016). These extreme conditions may lead U/LIRGs to convert gas into stars in a mode distinct from what we find in main-sequence galaxies like the Milky Way (e.g., Daddi et al. 2010; Genzel et al. 2010), and almost certainly lead to a higher rate of star formation per unit gas mass. The combination of high opacity, high gas surface density, and on-going star formation also makes these galaxies key testbeds for theories exploring the balance between feedback and gravity (e.g., Murray et al. 2005; Shetty et al. 2011). Indeed, for example,Thompson et al. (2005) have argued that the most extreme local U/LIRGs may represent "Eddington limited" star-forming systems or "maximal starbursts", which produces stars at the maximum capacity allowed for the considered feedback mechanism

Assessing the physics of U/LIRGs requires knowing their intensive properties, i.e., the luminosity or mass per unit area or volume. The extremity of these systems emerges most clearly when their high luminosities is viewed in the context of the very small area from which it emerges. In turn, measuring these intensive quantities requires knowing the size of the region where star formation is on-going. This is a challenging measurement. Even the most nearby U/LIRGs are quite distant (50-150 Mpc) compared to prototypes of more quiescent "normal" galaxies. Thus high very spatial resolution is required to study the compact energetically dominant regions at the heart of these systems. Compounding the challenge, U/LIRGs host enormous amounts of dust (e.g., Av 1000 for Arp 220 Lutz et al. 1996), rendering 
them optically thick at optical and possibly even infrared wavelengths. They are also opaque at very long radio wavelengths due to free-free absorption (e.g., Condon et al. 1990), leaving them transparent only over a limited regime, from radio to submillimeter wavelengths (see Barcos-Muñoz et al. 2015, for the extreme case of Arp 220).

Interferometric radio imaging is the ideal, and almost only, way to measure the sizes of the energetically dominant regions at the heart of local U/LIRGs. Radio interferometers make it possible to achieve the high angular resolution required to resolve the compact central starbursts, while the $\mathrm{cm}$ wavelength regime allows us to see through the enormous dust columns that prevents the measurements of the inner disk at optical wavelengths. The two dominant radio continuum emission mechanisms at cm wavelengths, free-free ("thermal") and synchrotron ("nonthermal") emission, both trace the distribution of recent star formation.

Condon et al. (1990) and Condon et al. (1991b) use $1.49 \mathrm{GHz}$ (up to 1".5 of angular resolution) and $8.44 \mathrm{GHz}$ (up to 0.25 of angular resolution) continuum emission measured with the Very Large Array (VLA), to study the energetically dominant regions in U/LIRGs. They provided what are still some of the strongest constraints on the sizes of the star-forming/AGN dominated regions in these systems. Because the VLA has fixed antenna configurations, higher frequency observations provide better angular resolution. However, because galaxies are fainter at high frequency and the sensitivity of the VLA receivers has been lower, efforts to push high resolution imaging of these systems beyond $\nu \sim 10 \mathrm{GHz}$ have been limited.

With the upgrade from the VLA to the Karl G. Jansky Very Large Array (VLA), this has changed. Both bandwidth and receiver sensitivity improved, making the VLA at high frequencies a powerful tools for imaging the radio continuum at very high spatial resolution. In particular, the Ka band $(26.5-40 \mathrm{GHz})$ has the perfect balance between transparency to the radio emission, high spatial resolution, and sensitivity, making it the ideal frequency range to study local U/LIRGs. We demonstrated this capability in Barcos-Muñoz et al. (2015), where we used the VLA at Ka band to make the sharpest full-flux recovery image to date of the nuclear disks of Arp 220.

Here we extend the work of Barcos-Muñoz et al. (2015) to a sample of 22 of the 
most luminous northern U/LIRGs. This is the first high resolution, high sensitivity, $33 \mathrm{GHz}$ continuum survey of local U/LIRGs. The angular resolution of the VLA at $\nu=33 \mathrm{GHz}$ improves on that of the $8.44 \mathrm{GHz}$ of Condon et al. (1991b), still the best results to date, by a factor of two.

The paper proceeds as follows. In Section 3.2, we describe the survey and the data reduction process. In Section 3.3, we report on the measurements that we performed. The physical implications of these measurements are explored in Section 3.4. In Section 3.5, we discuss the nature of the energy emission at $33 \mathrm{GHz}$, the implied physical conditions in these systems, the implications these measurements have on star formation scaling relations, and whether the systems in our sample are maximal starbursts. We end with concluding remarks in Section 3.6. In the Appendix, we present detailed notes on individual systems.

Throughout this paper, we adopt $\mathrm{H}_{0}=73 \mathrm{~km} \mathrm{~s}^{-1} \mathrm{Mpc}^{-1}, \Omega_{\text {vacuum }}=0.73$ and $\Omega_{\text {matter }}=0.27$, with velocities corrected to the cosmic microwave background (CMB) frame.

\subsection{Sample, Observations, and Data Reduction}

We used the Karl G. Jansky Very Large Array (VLA) to observe radio continuum emission from the most luminous nearby LIRGs and ULIRGs. Our sample (see Table 3.1) consists of 22 sources from the IRAS Revised Bright Galaxy Sample (RBGS; Sanders et al. 2003). These galaxies have infrared luminosities $\mathrm{L}_{\mathrm{IR}}[8-1000 \mu \mathrm{m}]$ $=10^{11.6}-10^{12.6} \mathrm{~L}_{\odot}$ and were selected to be northern enough to be observed by the VLA, i.e., $\delta>-15^{\circ}$. These systems are also a subset of the Great Observatories All-sky LIRG Survey (GOALS; Armus et al. 2009), for which multiwavelength data are available.

As part of the resident shared risk project AL746, we observed the radio continuum emission from each source at $\mathrm{C}$ band (4-8 GHz) and $\mathrm{Ka}$ band (26.5-40 GHz). For each observation we used dual polarization mode with two $1 \mathrm{GHz}$-wide bands. Each band was split into eight $128 \mathrm{MHz}$ spectral windows (spw's) with 64 channels each. 


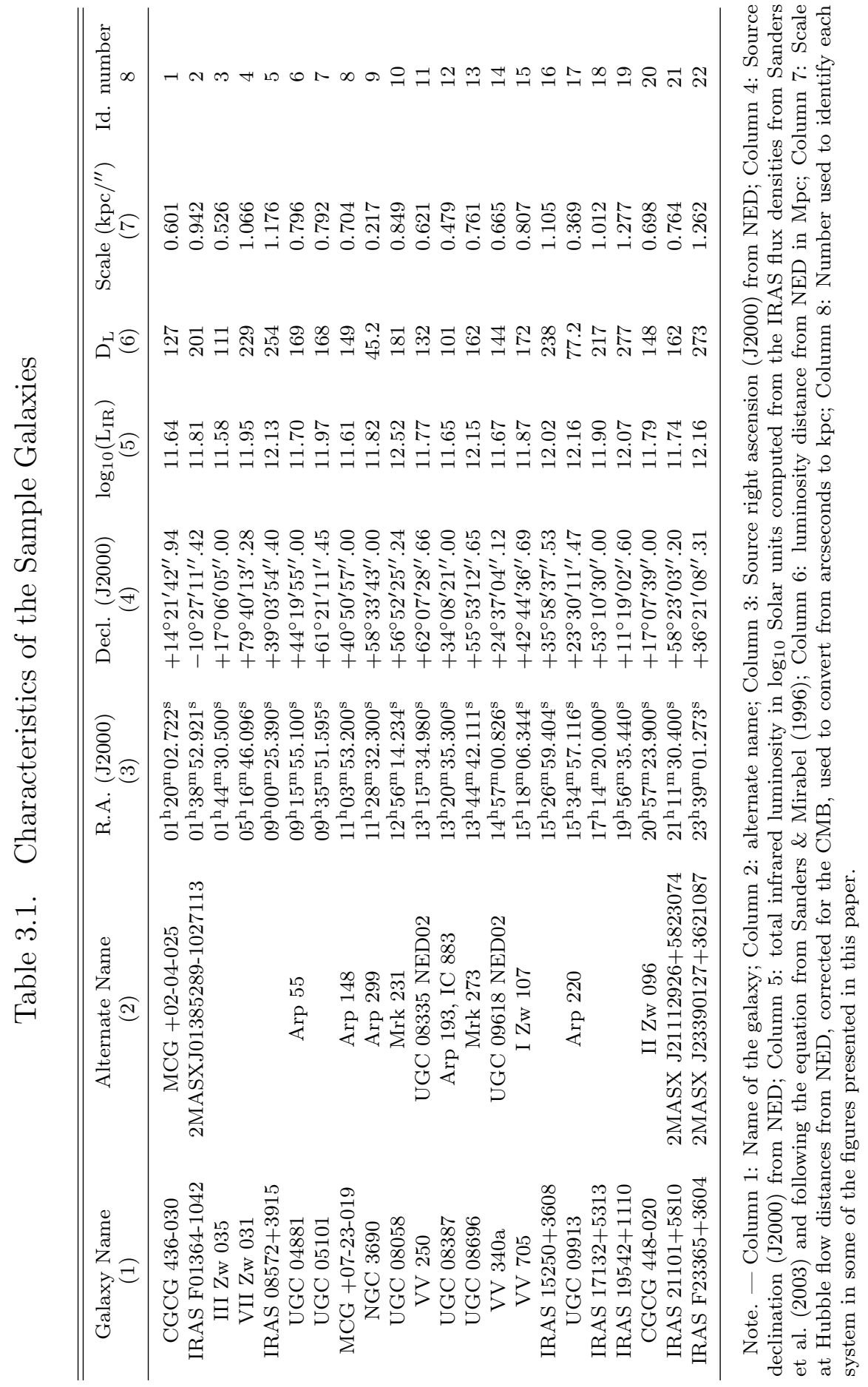


We centered the $1 \mathrm{GHz}$ bands at $\sim 4.7$ and 7.2 in $\mathrm{C}$ band and $\sim 29$ and $36 \mathrm{GHz}$ in Ka band.

In order to recover emission across a wide range of spatial scales, we observed our sample in each frequency range in separate sessions using each of the four VLA configurations of the array (A, B, C and D, from highest to lowest spatial resolution) over the period 2010 August 2 to 2011 August 16. In the D and C configurations, we observed each source for five minutes. In the B configuration, we observed each source for ten minutes split between two five minute scans. In the A configuration we observed most sources for 20 minutes, split into five minute scans. Due to scheduling constraints, eight sources were not observed in the A configuration at Ka band; these are identified with an asterisk in Table 3.3. Thus the total time on source for most targets was $\sim 40$ minutes per band, though the time on sky was larger by a factor of $\approx 2-3$ due to high overheads during the Jansky VLA upgrade.

Leroy et al. (2011) presented first results from our observations at both bands using the $\mathrm{C}$ and D configurations. In this paper, we report on the full survey, emphasizing the Ka band observations and the combination of all four array configurations. These represent the highest resolution, highest sensitivity radio data for these galaxies in the literature. The $\mathrm{C}$ band observations combining all four array configurations will be reported in an upcoming paper focused on the resolved spectral energy distribution (Barcos-Muñoz et al. in preparation).

At the beginning of each session, we observed a flux density calibrator, either 3C48 or 3C286, which was also used to calibrate the bandpass. Through the rest of the session we alternated between observations of the science target and a phase calibrator within a few degrees of each science target, which we used to measure phase variations due to atmospheric/ionospheric fluctuations. Table 3.2 summarizes the calibrators used for each science target.

We used the Common Astronomy Software Application (CASA, McMullin et al. 2007) to calibrate, inspect, and analyze the data. We individually calibrated each 1 GHz-wide frequency range, or baseband, for each observing session. Before doing so, we reduced the volume of the data to speed up the calibration process by averaging the 
Table 3.2. Summary of the Observations

\begin{tabular}{|c|c|c|}
\hline $\begin{array}{c}\text { Galaxy Name } \\
\text { (1) }\end{array}$ & $\begin{array}{c}\text { Primary Calibrator } \\
(2)\end{array}$ & $\begin{array}{c}\text { Secondary Calibrator Ka band } \\
(3)\end{array}$ \\
\hline CGCG 436-030 & $3 \mathrm{C} 48$ & $\mathrm{~J} 0117+1418$ \\
\hline IRAS F01364-1042 & $3 \mathrm{C} 48$ & J0141-0928 \\
\hline III Zw 035 & $3 \mathrm{C} 48$ & $\mathrm{~J} 0139+1753$ \\
\hline VII Zw 031 & $3 \mathrm{C} 48$ & $\mathrm{~J} 0410+7656$ \\
\hline IRAS $0857+3915$ & $3 \mathrm{C} 286$ & $\mathrm{~J} 0916+3854$ \\
\hline UGC 04881 & $3 \mathrm{C} 286$ & J0920+4441 \\
\hline UGC 05101 & $3 \mathrm{C} 286$ & $\mathrm{~J} 0921+6215$ \\
\hline $\mathrm{MCG}+07-23-019$ & $3 \mathrm{C} 286$ & $\mathrm{~J} 1101+3904$ \\
\hline NGC 3690 & $3 \mathrm{C} 286$ & $\mathrm{~J} 1128+5925$ \\
\hline Mrk 231 & $3 \mathrm{C} 286$ & $\mathrm{~J} 1302+5748$ \\
\hline VV 250 & $3 \mathrm{C} 286$ & $\mathrm{~J} 1302+5748$ \\
\hline UGC 08387 & $3 \mathrm{C} 286$ & $\mathrm{~J} 1317+3425$ \\
\hline UGC 08696 & $3 \mathrm{C} 286$ & $\mathrm{~J} 1337+5501$ \\
\hline VV 340a & $3 \mathrm{C} 286$ & $\mathrm{~J} 1443+2501$ \\
\hline VV 705 & $3 \mathrm{C} 286$ & $\mathrm{~J} 1521+4336$ \\
\hline IRAS $15250+3609$ & $3 \mathrm{C} 286$ & $\mathrm{~J} 1522+3144$ \\
\hline Arp 220 & $3 \mathrm{C} 286$ & $\mathrm{~J} 1539+2744$ \\
\hline IRAS $17132+5313$ & $3 \mathrm{C} 286$ & $\mathrm{~J} 1740+5211$ \\
\hline IRAS $19542+1110$ & $3 \mathrm{C} 48$ & $\mathrm{~J} 1955+1358$ \\
\hline II Zw 096 & $3 \mathrm{C} 48$ & $\mathrm{~J} 2051+1743$ \\
\hline IRAS $21101+5810$ & $3 \mathrm{C} 48$ & $\mathrm{~J} 2123+5500$ \\
\hline IRAS F23365+3604 & $3 \mathrm{C} 48$ & $\mathrm{~J} 2330+3348$ \\
\hline
\end{tabular}

Note. - Column 1: Name of the galaxy; Column 2: Primary calibrator used for the observations; Column 4: Secondary calibrator for Ka Band observations 
data in time. We used a time interval that depends on the configuration, smoothing the data to an integration length of $10 \mathrm{~s}(\mathrm{D}), 5 \mathrm{~s}(\mathrm{C}), 2 \mathrm{~s}(\mathrm{~B})$, or $1 \mathrm{~s}(\mathrm{~A})$. This was necessary since the correlator integration time was limited to $1 \mathrm{~s}$ at the time of these observations. We then Hanning smoothed the data in order to minimize Gibbs ringing effects, if present. Before calibration, we also applied standard flagging to remove shadowed antennas, a few channels at the edge of each spw, and other pathological data.

We began the calibration by solving for the bandpass response and flux density scale using observations of $3 \mathrm{C} 48$ or $3 \mathrm{C} 286$. At the time, we adopted the flux density for these sources on the "Perley-Butler 2010" scale, assuming that the Ka band emission shares the same structure as the VLA-provided Q-band model. We input these choices into our data set using the CASA task setjy and accounting for channel to channel variations in the model flux due to the spectral index of our sources.

Once setjy was applied, we used the CASA task gaincal to solve for short timescale $(\sim 10 \mathrm{~s})$ corrections to the phase response of each antenna during the bandpass calibrator observation. We then solved for the phase and amplitude response of each antenna as a function of frequency using the task bandpass. We derived corrections for each channel and each antenna (bandtype='B'), solving for a single correction that we assumed to span the entire length of the observation (parameters combine='scan' and solint='inf' in bandpass). Before solving, we applied the short-timescale phase corrections.

We assessed the quality of the bandpass solutions by inspecting the solutions for phase and amplitude as a function of frequency. Then, we applied the bandpass solution to the bandpass calibrator only and inspected the calibrated data. We looked for pathological channels, antennas, or baselines. When we found these, we added them to our master list of data to flag. We then reset the entire calibration process, applied all flagging identified to date, and then repeated the calibration using only non-pathological data. We iterated until the corrected bandpass calibrator data showed no evidence for corrupted features. At this point, we applied the flags and the bandpass solution to the entire data set, including the secondary calibrators and target sources. This also sets the flux scale of the data, because we specified the true 
flux of the source (according to the VLA standard) using setjy.

Following these, we calibrated the time dependent amplitude and phase response of each antenna using observations of the secondary calibrators. We solved for the phase response on two different timescales, averaged over a whole scan (solint='inf') and over a shorter timescale of $\approx 20 \mathrm{~s}$. We solved for amplitude corrections on a scan-long timescale, after first applying the short timescale phase calibration table to account for any possible loss of amplitude due to decorrelation. Then, comparing the corrected flux densities of our secondary calibrator to the known flux density of the bandpass calibrator, we derived the true flux density value for the secondary calibrators by using fluxscale.

We applied these corrections to the calibrators and inspected their phase and amplitude as a function of time, $u-v$ distance, frequency, and baseline. When necessary, we flagged further data and iterated on the phase and amplitude calibration process. Once no more flagging appeared necessary, we applied the corrections to the science targets, which we split out from the main data sets for imaging.

Once the data had been calibrated, we imaged each science target. To do this, we used the task CLEAN in mode mfs (Sault \& Wieringa 1994), with Briggs weighting setting robust $=0.5$. For each array configuration, we imaged each baseband independently. Whenever possible, we iterated this imaging with phase and amplitude self calibration. We typically performed $\sim$ four iterations, though this varied according to the signal-to-noise of the data - from no self calibration up to eight iterations. After several iterations of phase-only self calibration, and when possible, we also performed amplitude self calibration. We always constrained these to be only relative solutions among the antennas (solnorm=True in CASA's gaincal).

After self calibrating the two basebands independently, we combined both into a single image using nterms $=2^{1}$ in clean. This yielded four images per source (one per array configuration). Finally, we jointly imaged all self-calibrated data, combining all eight measurement sets (four configurations and two basebands). This combined image represents our best data product, combining all of our observations with sen-

\footnotetext{
${ }^{1}$ This allows us to model the frequency dependence of the sky emission with two Taylor coefficients. In this mode clean delivers total intensity and spectral index images.
} 
sitivity to a wide range of spatial scales. In the highest signal to noise cases, for example UGC 08058 (Mrk 231) and UGC 09913 (Arp 220), we performed further self calibration during this final imaging step.

These final images have a nominal frequency $\nu=32.5 \mathrm{GHz}$ and a typical rms noise $26 \mu \mathrm{Jy} \mathrm{beam}^{-1}$. Table 3.3 reports the exact beam size and rms noise for the combined image for each target.

\subsubsection{Additional Data}

To help interpret our results, we use observations of our sample at $1.49 \mathrm{GHz}$ (beam FWHM $\left.\sim 15^{\prime \prime}\right)$ from Condon et al. (1990). We also use the $5.95 \mathrm{GHz}$ flux densities (beam FWHM $\sim 0.4^{\prime \prime}$ ) from Leroy et al. (2011) and the CO flux densities, obtained using the ARO 12-m antenna (FWHM = 1 arcminute), which will be reported in Privon et al. (in preparation). We present a compilation of the flux densities at these different frequencies, along with the $32.5 \mathrm{GHz}$ flux densities in Table 3.3. The uncertainties of the $1.49 \mathrm{GHz}$ flux density values are assumed to be dominated by flux density calibration errors ( 5\%, see Section III in Condon et al. (1990)), considering the extension of the emission at $1.49 \mathrm{GHz}$ is mostly point-like for our sample.

There are five sources without literature values at $1.49 \mathrm{GHz}$, but at slightly lower frequencies. These are VII Zw 031, II Zw 096 and IRAS F23365+3608 with values at $1.4 \mathrm{GHz}$ from the NVSS catalog (Condon et al. 1998), and IRAS 19542+1110 and IRAS $21101+5810$ with values at $1.425 \mathrm{GHz}$ from Condon et al. (1996). We assigned a conservative uncertainty of $10 \%$ to those values in order to account for the difference in frequency.

\subsection{Results}

In Figure 3.1, contour maps show new VLA $\nu=32.5 \mathrm{GHz}$ images for our sample of 22 local U/LIRGs. These are the first images of these systems that have both high resolution and sensitivity to a wide range of spatial scales. We use these images to measure: (1) the area of the energetically dominant region in each galaxy, (2) the 


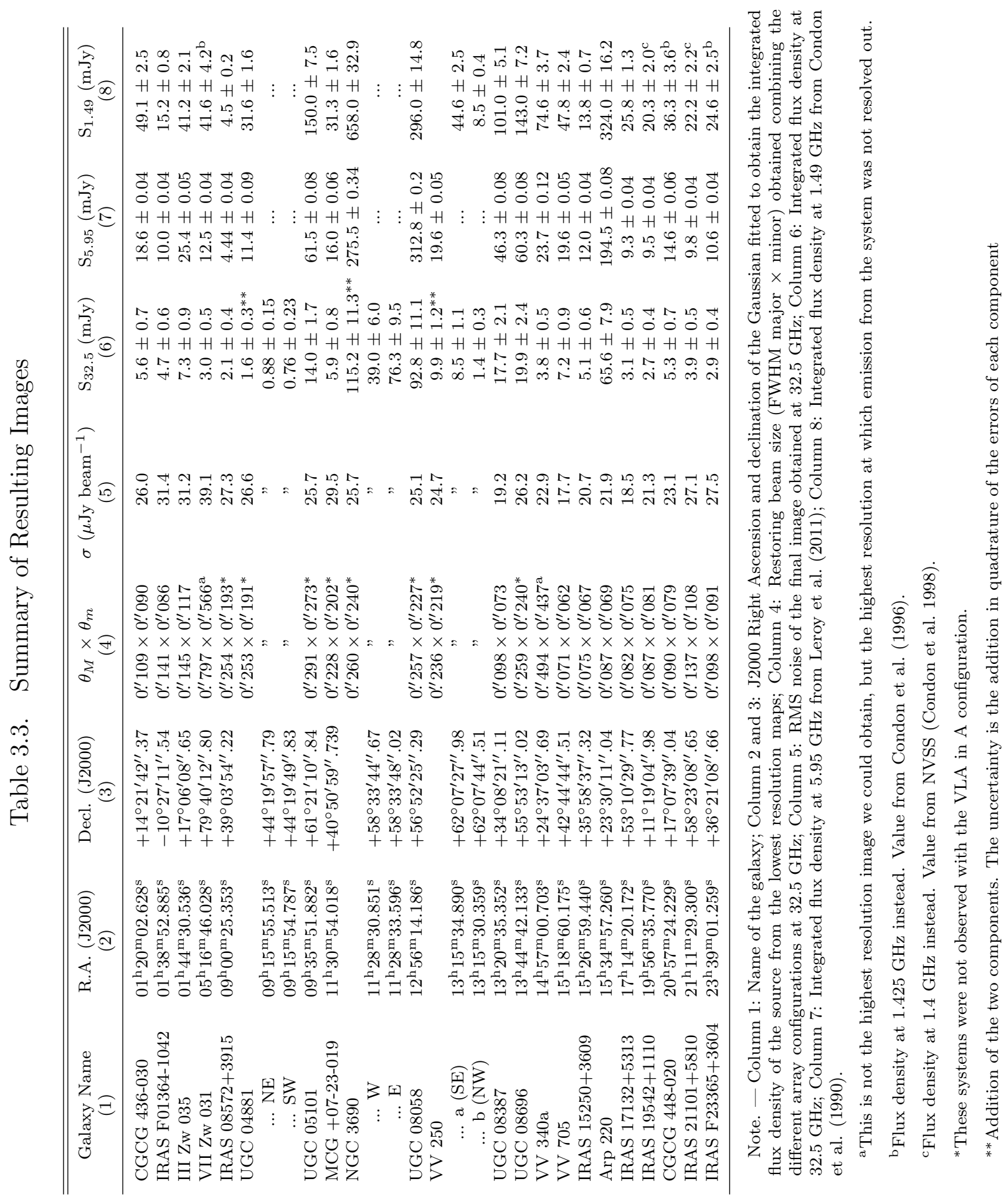


integrated flux density of each target at $32.5 \mathrm{GHz}$, and (3) the area and flux density contribution of compact regions to the integrated properties of each system. In Tables 3.3 and 3.4 we report values from (1) and (2), and data from the literature which we use to constrain the origin of the radio emission (from the spectral index) and the nature of the emission powering the energetics of each system.

\subsubsection{Flux Densities at $\nu=32.5 \mathrm{GHz}$}

We measure integrated flux densities for each source from our lowest spatial resolution observations, which were obtained in the VLA's D configuration. We expect that the maximum recoverable scale for the $\mathrm{D}$ configuration, $\approx 22^{\prime \prime}$, recovers all emission from most of our sources. This corresponds to $\sim 16 \mathrm{kpc}$ at the $165 \mathrm{Mpc}$ median distance of our sample, which is much larger than the median sizes for a large sample of local $\mathrm{U} /$ LIRGs (normally $\leq 1.5 \mathrm{kpc}$ ) derived by Liu et al. (2015) based on $1.49 \mathrm{GHz}$ radio continuum observations and on near-IR data (normally $\leq 2.2 \mathrm{kpc}$ for well separated pairs $^{2}$ ) derived by Haan et al. (2011) based on HST NICMOS data.

Most of the galaxies in our sample show point-like emission in the $\mathrm{D}$ configuration only observations, which have beam size $\approx 2.7^{\prime \prime}$. We obtained the flux densities of these sources by fitting a Gaussian using the CASA task imfit. Other targets, including Arp 299, II Zw 96, IRAS F17132+5313, VII Zw 031, VV 250 and VV 705, showed some extent - or more than one component - in the D configuration maps. In these cases, we tapered the $\mathrm{D}$ configuration data to a lower resolution until the morphology became a single point-like source. Then we fit a Gaussian to this degraded image. Two cases, Arp 299 and VV250, show separated components that could only be fit using two Gaussians, even in the tapered images; we report the sum of the components in the integrated flux density.

The uncertainties that we report sum (in quadrature) the statistical error calculated by the imfit task with the flux density calibration uncertainty at Ka band ( $12 \%$ calculated in Barcos-Muñoz et al. (2015)). For the two faintest galaxies in our sample, UGC 04881 and IRAS 08572+3015, the signal-to-noise of the D configuration

\footnotetext{
${ }^{2}$ This number decreases by a factor of 3 or more for mergers that are closer together.
} 
map was not high enough to recover their integrated flux density. For these systems, we instead report results from the map combining data from all configurations; we tapered this combined map until we recovered a point-like structure and then fit one, or two, Gaussians, as with the other sources.

\subsubsection{Spectral Indices Involving $\nu=32.5 \mathrm{GHz}$}

In addition to our measured flux densities, Table 3.3 reports literature flux densities for our sources at $\nu=1.49$ and $5.95 \mathrm{GHz}$. We combine these with our $\nu=32.5 \mathrm{GHz}$ measurements to calculate galaxy-integrated spectral index between $1.49 \mathrm{GHz}$ and $5.95 \mathrm{GHz}\left(\alpha_{1.5-6}\right)$ and between $5.95 \mathrm{GHz}$ and $32.5 \mathrm{GHz}\left(\alpha_{6-33}\right)$, where $\mathrm{S}_{\nu} \propto \nu^{\alpha}$.

In Figure 3.2, we show the derived spectral indices, plotting $\alpha_{1.5-6}$ as a function of $\alpha_{6-33}$. Here the solid line shows equal spectral indices for both pairs of bands, which we would expect if a single spectral index holds across the entire radio regime (from 1.5 to $33 \mathrm{GHz}$ ). Dashed lines show $\alpha=-0.8$, a typical spectral index for synchrotron emission without any opacity effects (e.g., Condon 1992).

For the five sources without measurements at $1.49 \mathrm{GHz}$ (see Section 3.2.1), we assume $\alpha_{1.5-6} \approx \alpha_{1.4-6} \approx \alpha_{1.43-6}$. This is a safe assumption considering $\alpha_{1.5-6}<$ -1.0 for our sample (see Figure 3.2) and that we conservatively overestimated the

uncertainty in the measurement to account for the change in the SED between the different frequencies and $1.49 \mathrm{GHz}$. 

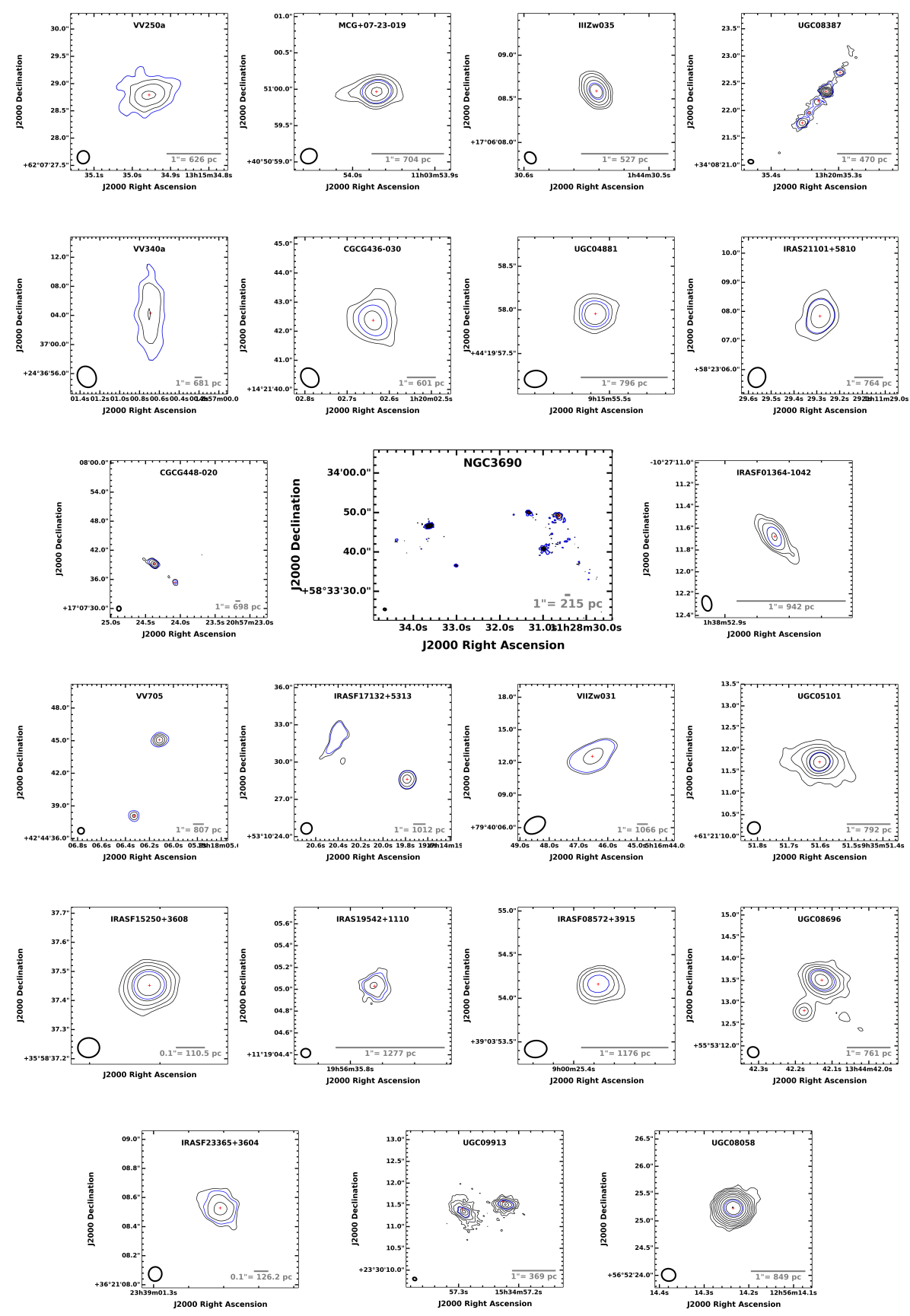

Fig. 3.1.- : Contour maps of the $32.5 \mathrm{GHz}$ continuum emission of each galaxy in our sample used to determine $\mathrm{A}_{50}$ (area of the contour enclosing $50 \%$ of the total flux density at $32.5 \mathrm{GHz}, \mathrm{C}_{50}$, shown in blue). The contours are in factor of two steps with the outermost corresponding to 5 times the rms of the map, $\sigma_{\mathrm{A}_{50}}$, where the mean value for our sample is $33 \mu \mathrm{Jy}$ beam $^{-1}$. In each map, we show the beam (black) in the bottom left corner and a scale bar of $1^{\prime \prime}$ in size (with its equivalent in parsec) in the bottom right corner. This scale bar is $0^{\prime \prime} 1$ in size for IRAS $15250+3608$ and IRAS F23365+3604. The red crosses indicate the location of compact sources whose properties were derived from Gaussian fits (see Section 3.3.4). Most of the emission in our sample is compact, with only a few systems showing considerable extended emission (e.g., VV 340a) and others showing a combination of compact and extended emission (e.g., UGC 08387). 

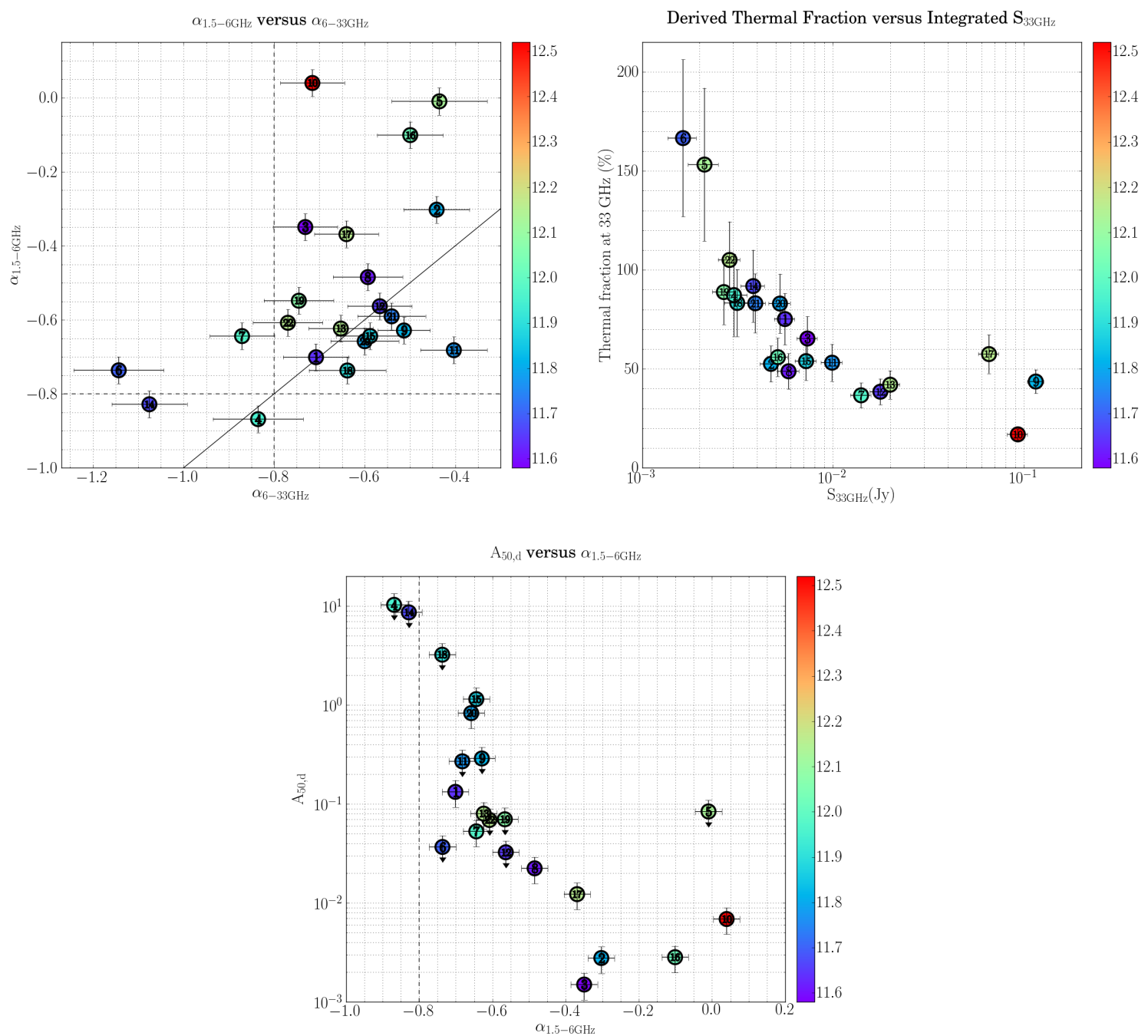

Fig. 3.2.-: Assessment of the nature of the energy source at $33 \mathrm{GHz}$. The systems are labeled by their Id. number and color coded by their infrared luminosity (TopLeft) $\alpha_{1.49-6 \mathrm{GHz}}$ versus $\alpha_{6-33 \mathrm{GHz}}$ values using integrated flux density measurements at $1.49 \mathrm{GHz}$ from Condon et al. (1990) and at $6 \mathrm{GHz}$ from Leroy et al. (2011). The solid line shows a linear correlation, and the dashed lines indicate a typical synchrotron emission slope of -0.8. The median value for $\alpha_{1.49-6 \mathrm{GHz}}$ is -0.62 and -0.64 for $\alpha_{6-33 \mathrm{GHz}}$. More than half of the sources show a change in slope at 6 $\mathrm{GHz}$, becoming steeper at higher frequencies. This is most likely due to a change in the optical depth towards higher frequencies, where the source becomes more optically thin. There are three sources (\#9, \#11 and \#18) that show instead a slightly flatter spectral index at higher frequencies. These changes in slope are most likely due to thermal emission contribution becoming important at higher frequencies. Only one source (\#10, Mrk 231) shows an inversion in the spectral index, indicating there is a change from an optically thick to an optically thin regime between 1.5 and $33 \mathrm{GHz}$. (TopRight) Predicted thermal fraction at $33 \mathrm{GHz}$, from IR luminosity, versus integrated flux density at $33 \mathrm{GHz}$. Most of the systems show thermal fractions of $\geq 50 \%$, in agreement with SED models (Condon \& Yin 1990; Condon 1992). (Bottom) Half-light area as a fucntion of $\alpha_{1.49-6 \mathrm{GHz}}$. There is a tentative correlation of flatter spectral index in the range 1.5 to $6 \mathrm{GHz}$ for more compact sources. This is expected since more compact sources are more obscured and then subject to more free-free absorption at low frequencies. 


\subsubsection{Size of the Radio Emission}

A main goal of our survey is to estimate the extent of the radio continuum emission in our targets with the goal of constraining the size of the energetically dominant region in these distant, compact, heavily obscured systems. To do this, we analyzed the final images combining data from all the array configurations.

These high resolution images are sensitive to the brightest compact cores, but they have lower surface brightness sensitivity than the D configuration data used for the total flux density. Therefore, they may miss extended, low surface brightness emission. To take this into account, we measure the size of the energetically dominant region from the half light area $\left(A_{50}\right)$. This is the area enclosed by the highest intensity isophote that encloses half of the integrated flux density of the system (measured from the lower resolution data above).

We require the intensity of the isophote enclosing the half-light radius, $\mathrm{C}_{50}$, to be at least 5 times the rms noise in the image. If $\mathrm{C}_{50}$ would be less than $5 \sigma$ in the combined image, we interpret this to indicate an important component of extended, low surface brightness emission. In order to recover this emission, we measure $\mathrm{A}_{50}$ for these systems from lower resolution versions of the data with better surface brightness sensitivity. First, we tried using natural weighting instead of briggs (see Section 3.2). If this still did not recover half the light in a $\mathrm{S} / \mathrm{N}>5$ contour, then we tried images with progressively lower resolution images by $u-v$-tapering the data. We stepped the size of the taper by $0^{\prime \prime} 2$ and used briggs weighting schemes with robust parameter 0.5 . In this way, we measure $A_{50}$ from the highest resolution image where $\mathrm{C}_{50}$ can be reliably measured.

The following systems required $u-v$-tapering: CGCG 436-030, CGCG 448-020, IRAS 21101+5810, IRAS F17132+5313, VV 340a and VV 705. For NGC 3690, the natural weighting approach was enough.

Once we identified a reliable $\mathrm{C}_{50}$ value, we calculated the observed $\mathrm{A}_{50}$ by multiplying the number of pixels within $\mathrm{C}_{50}$ by the pixel area. Figure 3.1 shows the images that were used to measure $A_{50}$ and the $C_{50}$ contour (in blue) for each source.

This approach measures $A_{50}$ for the source convolved with the telescope beam. 
Many of our sources have areas close to that of the synthesized beam. We show this in Figure 3.3. There, we plot the observed $\mathrm{A}_{50}$ as a function of the beam area, $\mathrm{A}_{\text {beam }}{ }^{3}$, in units of $\operatorname{arcsec}^{2}$ (top-left panel) and $\mathrm{kpc}^{2}$ (top-right panel).

Note that the quantity of physical interest is the true size of the $32.5 \mathrm{GHz}$ emission with the beam deconvolved, $\mathrm{A}_{50 \mathrm{~d}}$. The solid line in the top panels of Figure 3.3 shows the limit where the source shows the same area as the beam, $A_{\text {beam }}=A_{50}$ (solid line). A dashed line indicates where $\mathrm{A}_{50}=2 \times \mathrm{A}_{\text {beam }}$, which we consider a practical threshold for the emission to be resolved. We view the sources lying between the solid and dashed lines as marginally resolved. We assumed their intrinsic shape (deconvolved size) follows a Gaussian distribution. In these cases, the deconvolved size of the sources is calculated by $A_{50}$ (deconvolved $)=A_{50}$ (observed) $-A_{\text {beam }} \equiv A_{50 d}$, equivalent to deconvolving the FWHM in quadrature.

For two sources, UGC 04881 and VV250, a second, faint component could only be recovered in the low resolution map used to assess the integrated flux density. In both cases, the components are unresolved in this integrated map. Here, we had to lower our conservative limit of $5 \sigma$ in order to recover the half-light area. In these two systems, we measure $\mathrm{C}_{50}$ from a contour with $S / N \approx 3$ and treat the size estimate as an upper limit (see Table 3.4).

In Figure 3.3, two sources lie below the solid line, indicating an observed size smaller than the beam. These are IRAS F08572+3915 and UGC 04881NE. Although statistical fluctuations could produce this situation, the data appear to be too high signal-to-noise for this explanation. The most likely culprit is a calibration issue when combining observations using the different array configurations. In order to be conservative, we adopt an upper limit of $A_{50 d}=A_{\text {beam }}$ for these systems.

On the other hand, there are several galaxies that are heavily resolved according to the top panel of Figure 3.3. In order to determine the best estimate of $\mathrm{A}_{50 d}$ for those sources, we inspected the shape of the $\mathrm{C}_{50}$ contour (blue in Figure 3.1) to determine if the source exhibits a Gaussian shape. If it does, then we apply the same approach used for the marginally resolved sources. This tends to be the case when more than

\footnotetext{
${ }^{3} \mathrm{~A}_{\text {beam }}=\frac{\pi \theta_{\operatorname{maj}} \theta_{\min }}{4 \ln (2)}$ with $\theta_{\text {maj }}$ and $\theta_{\text {min }}$ the FWHM of the synthesized beam along its major and minor axis.
} 

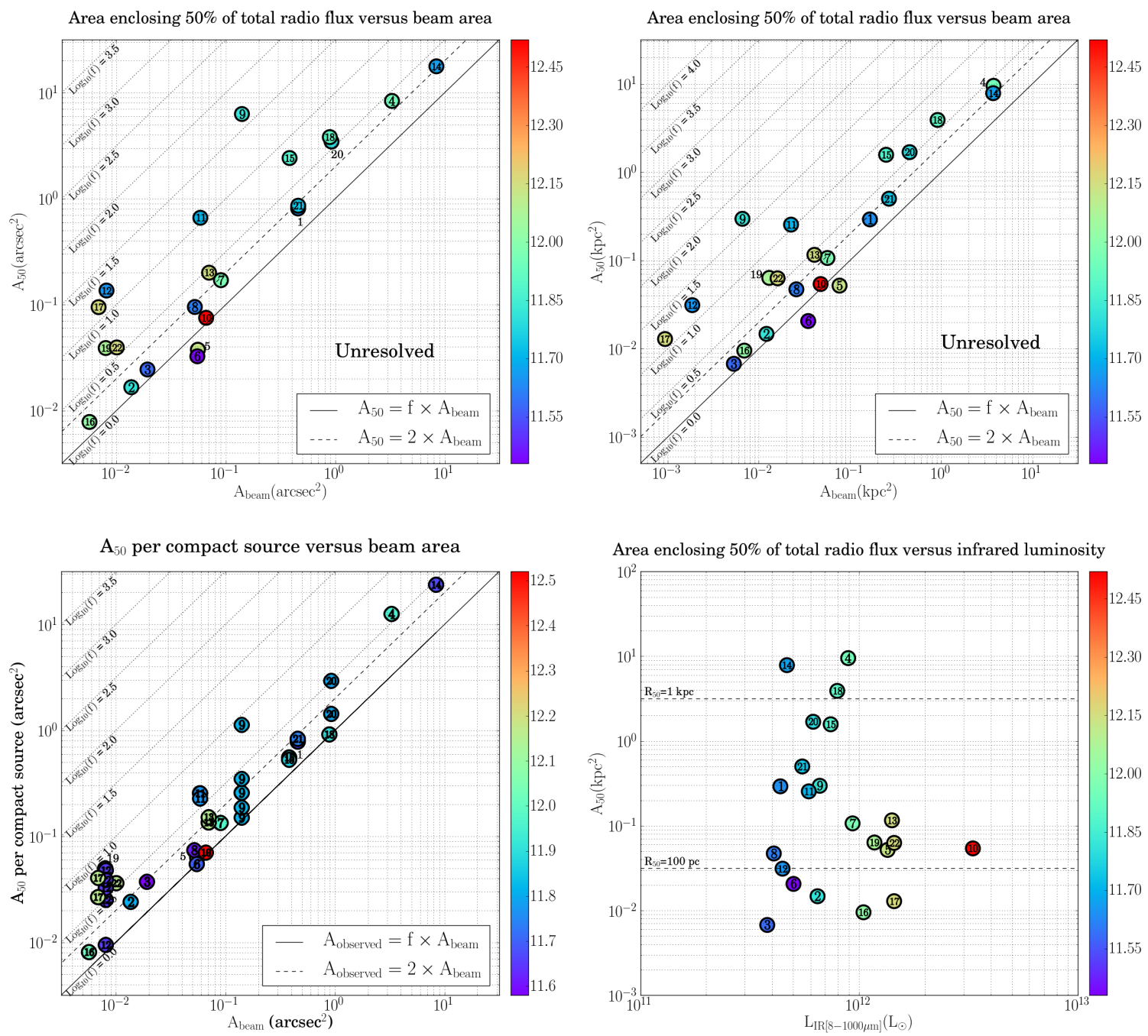

Fig. 3.3.- - Sizes of the radio emitting regions detected at $32.5 \mathrm{GHz}$ from our sample. The systems are labeled by their Id. number and color coded by their infrared luminosity (color bar), both shown in Table 3.1. (Top left) Observed $\mathrm{A}_{50}$ (area enclosed by the blue contours shown in the maps of Figure 3.1) versus beam area (see Section 3.3.3 for details) in $\operatorname{arcsec}{ }^{2}$. The solid line shows where $A_{50}=A_{\text {beam }}$, and the dashed line shows where $A_{50}=2 A_{\text {beam }}$. We only consider a source to be resolved if it lies above the dashed line, and partially resolved if it lies between the dashed and solid lines. The dotted lines show the beam size scaled by a constant starting at 1 (solid line) and incrementing in 0.5dex. (Top right) Same as in the previous panel, but using scales from Table 3.1 to convert from $\operatorname{arcsec}^{2}$ to $\mathrm{kpc}^{2}$. (Bottom left) Observed $\mathrm{A}_{50}$ for compact sources obtained from Gaussian fitting (see Section 3.3.4), as a function of beam area. (Bottom right) Observed $\mathrm{A}_{50}$ versus $\mathrm{L}_{\mathrm{IR}}$. The dashed lines show an area for an equivalent radius of 100 pc and $1 \mathrm{kpc}$ (see Section 3.3.3). Our survey is powerful and allow us to resolve or partially resolve all, but two of the sources in our sample. The nuclear star forming regions in our sample, as traced by the $32.5 \mathrm{GHz}$ continuum emission, show representative radii, $\mathrm{R}_{50}$, that go from $\sim 20 \mathrm{pc}$ to $1.7 \mathrm{kpc}$ in size, with most of the sources showing sizes that are comparable to large giant molecular clouds $(\sim 100 \mathrm{pc})$. The ULIRGs in our sample all show compact emission with equivalent radii that go from 30 to 160 pc. Combining the information from the panels in the left column we observe that the compactness of the emission is also evident from the contribution of compact regions with sizes several times smaller than the total $\mathrm{A}_{50}$ of the source. 
one component is present, such as VV 705 and CGCG 448-020. If $\mathrm{C}_{50}$ showed a more complex morphology, our simple deconvolution becomes invalid. In these cases we instead assume that the measured, not deconvolved $A_{50}$ is an upper limit to the true size. This is true for the following galaxies: IRAS 19542+1110, IRAS F23365+3604, UGC 08387, VII Zw 031, VV 250a and VV 340a.

For Arp 299 and IRAS F17132+5313, one component of the $\mathrm{C}_{50}$ contour shows a Gaussian distribution while others show more complex morphology. In both cases, we performed the deconvolution on the Gaussian components. Then we have a partially deconvolved estimate, $A_{50 \mathrm{~d}}<\mathrm{A}_{50}$ (observed), which is still an upper limit because of the un-deconvolved more complex structure. We report values for $\mathrm{A}_{50 d}$ and $\mathrm{C}_{50}$ in Table 3.4, along with an equivalent $R_{50 d}$ value where $A_{50 d}=\pi R_{50 d}^{2}$. We caution, however, that $R_{50 d}$ is only a representative number reflective of the upper limit to the area in these cases.

In Table 3.4, we also report the degree of Gaussianity, defined as the ratio between the flux density level of the $\mathrm{C}_{50}$ contour and the peak flux density. For a normal Gaussian this value should be 0.5 .

\subsubsection{Compact Sources Decomposition}

In addition to the integrated flux density and a characteristic size, we measured the contribution of compact sources to the overall flux density of our targets. For our purposes, compact sources are those that show distributions similar to a Gaussian and are tractable to being fit with the CASA task imfit. As Figure 4.4 shows, these are often, but not always, similar in size to the beam. We fit these sources and assess their contribution to the integrated luminosity.

Among our targets, only the north-east component in IRAS F17132+5313 shows no hint of emission distributed like a Gaussian. We do not fit a Gaussian to this source. On our other targets, we run imfit targeting a region chosen to enclose only the source of interest and to avoid other compact sources. We provided estimates of the rms of the image calculated from the emission-free pixels, and we began the fit with reasonable guesses when we needed to fit more than one Gaussian at a time 
Table 3.4. Summary of $32.5 \mathrm{GHz}$ emission sizes

\begin{tabular}{|c|c|c|c|c|c|c|}
\hline $\begin{array}{c}\text { Galaxy Name } \\
\text { (1) }\end{array}$ & $\begin{array}{c}\theta_{A_{50} M} \times \theta_{A_{50} m} \\
(2)\end{array}$ & $\begin{array}{c}\mathrm{C}_{50}\left(\sigma_{A_{50}}\right) \\
\quad(3)\end{array}$ & $\log _{10}\left(\mathrm{~A}_{50 d}\right)\left(\operatorname{arcsec}^{2}\right)$ & $\begin{array}{l}\mathrm{R}_{50 d}(\mathrm{kpc}) \\
(5)\end{array}$ & uplim? & $\begin{array}{c}\text { Gaussianity factor } \\
(7)\end{array}$ \\
\hline CGCG 436-030 & $0 . .705 \times 00^{\prime \prime} 573$ & 19.9 & -0.456 & 0.201 & no & 0.308 \\
\hline IRAS F01364-1042 & $0^{\prime \prime} 141 \times 00^{\prime \prime} 086$ & 40.7 & -2.533 & 0.029 & no & 0.447 \\
\hline III Zw 035 & $0^{\prime \prime} 145 \times 00^{\prime \prime} 117$ & 64.5 & -2.286 & 0.021 & no & 0.502 \\
\hline VII Zw 031 & $2^{\prime \prime} 060 \times 11^{\prime \prime} 415$ & 5.7 & 0.922 & 1.739 & yes & 0.463 \\
\hline IRAS $08572+3915$ & $00^{\prime \prime} 254 \times 00^{\prime \prime} 193$ & 39.7 & $-1.256^{\mathrm{a}}$ & 0.156 & yes & 0.535 \\
\hline \multicolumn{7}{|l|}{ UGC 04881} \\
\hline$\ldots \mathrm{NE}$ & $0^{\prime \prime} 253 \times 0 . \prime 191$ & 23.4 & $-1.260^{\mathrm{a}}$ & 0.105 & yes & 0.683 \\
\hline$\ldots \mathrm{SW}$ & $5 !^{\prime \prime} 049 \times 33^{\prime \prime} 543$ & 3.0 & $1.307^{\mathrm{a}}$ & 2.022 & yes & 0.229 \\
\hline UGC 05101 & $0^{\prime \prime} 291 \times 00^{\prime \prime} 273$ & 70.1 & -1.098 & 0.126 & no & 0.255 \\
\hline MCG +07-23-019 & $0^{\prime \prime} 228 \times 00^{\prime \prime} 202$ & 25.6 & -1.367 & 0.082 & no & 0.253 \\
\hline NGC 3690 & $0^{\prime \prime} 379 \times 00^{\prime \prime} 327$ & & $0.779^{\mathrm{b}}$ & 0.300 & yes & \\
\hline$\ldots \mathrm{W}$ & $"$ & 5.1 & 0.710 & 0.277 & yes & 0.024 \\
\hline$\ldots \mathrm{E}$ & $"$ & 20.2 & -0.052 & 0.115 & no & 0.030 \\
\hline $\begin{array}{l}\text { UGC } 08058 \\
\text { VV } 250\end{array}$ & $0^{\prime \prime} 257 \times 00^{\prime \prime} 227$ & 895.8 & -2.048 & 0.045 & no & 0.345 \\
\hline ... a $(\mathrm{SE})$ & $0^{\prime \prime} 236 \times 0^{\prime \prime} .219$ & 5.0 & -0.180 & 0.285 & yes & 0.109 \\
\hline$\ldots \mathrm{b}(\mathrm{NW})$ & $10^{\prime \prime} 71 \times 9^{\prime \prime} 340$ & 3.2 & $2.055^{\mathrm{a}}$ & 3.730 & yes & 0.058 \\
\hline UGC 08387 & $0^{\prime \prime} 098 \times 00^{\prime \prime} 073$ & 7.4 & -0.867 & 0.100 & yes & 0.026 \\
\hline UGC 08696 & $0^{\prime \prime} 259 \times 00^{\prime \prime} 240$ & 56.6 & -0.887 & 0.155 & no & 0.205 \\
\hline VV 340a & $2^{\prime \prime} 983 \times 2^{\prime \prime} 488$ & 5.0 & 1.249 & 1.581 & yes & 0.491 \\
\hline VV 705 & $0^{\prime \prime} 583 \times 0.0^{\prime \prime} 579$ & 5.0 & 0.218 & 0.585 & no & 0.071 \\
\hline IRAS $15250+3609$ & $0 . .075 \times 0.0^{\prime \prime} 067$ & 52.5 & -2.672 & 0.029 & no & 0.366 \\
\hline Arp 220 & $0 . .087 \times 0.0^{\prime \prime} 069$ & 45.3 & -1.057 & 0.062 & no & 0.132 \\
\hline IRAS $17132+5313$ & $0 . .911 \times 0 . .864$ & 5.8 & 0.465 & 0.975 & yes & 0.259 \\
\hline IRAS $19542+1110$ & $0 . .087 \times 0.0^{\prime \prime} 081$ & 7.6 & -1.410 & 0.142 & yes & 0.342 \\
\hline CGCG 448-020 & $0^{\prime \prime} 970 \times 00^{\prime \prime} 841$ & 6.9 & 0.207 & 0.500 & no & 0.141 \\
\hline IRAS $21101+5810$ & $0^{\prime \prime} 686 \times 0.0^{\prime \prime} 590$ & 11.1 & -0.399 & 0.272 & no & 0.293 \\
\hline IRAS F23365+3604 & $=0.098 \times 0.0^{\prime \prime} 091$ & 6.8 & -1.405 & 0.141 & yes & 0.259 \\
\hline
\end{tabular}

Note. - Column 1: Name of the galaxy; Column 2: beam size at Ka band (32.5 GHz) of the image used to obtain $\mathrm{A}_{50}$ (see Section 3.3.3); Column3: Contour level enclosing $50 \%$ of the total flux density of the system in units of $\sigma_{A_{50}}$, the rms of the final image used to measure $\mathrm{A}_{50}$; Column 4: Best estimate of the deconvolved area enclosing $50 \%$ of the total emission at $32.5 \mathrm{GHz}, \mathrm{A}_{50 d}$ (see Section 3.3.3); Column 5: Equivalent circular radius of $\mathrm{A}_{50 \mathrm{~d}}$, i.e., assuming $\mathrm{A}_{50 \mathrm{~d}} \equiv \pi \mathrm{R}_{50 \mathrm{~d}}^{2}$; Column 6: If "yes", the value in Column 4 is an upper limit either because the emission is extended and applying a Gaussian deconvolution was not a good approximation (see Section 3.3.3), or because is unresolved; Column 7: Ratio of the flux density of the isophote enclosing 50\% of the total flux density at $32.5 \mathrm{GHz}$ to the peak flux density of the emission. For a Gaussian-like distribution this number is 0.5 .

${ }^{\mathrm{a}} \mathrm{A}_{50}$ for this galaxy is smaller than the beam size, so we adopted the beam area as the best estimate for its size.

${ }^{\mathrm{b}} \mathrm{A}_{50}$ for this galaxy is the addition of both components. 
(e.g., Arp 299, UGC 08696, VV 705). For UGC 08387, most of the compact sources have low signal-to-noise, so we fit each component individually, making use of one region at a time.

For the cases of the faint components in the systems UGC 04881 and VV250, the Gaussian fit was performed on the low resolution image that was used to obtain the integrated flux density of the system since it was only in those maps where the faint sources were recoverable. We report our findings in the bottom left panel of Figure 3.3 and in Figure 4.4.

\subsection{Implications of the Radio Sizes}

Our $33 \mathrm{GHz}$ images either measure or strongly constrain the size of the energetically dominant region in our targets. Radio observations are unique in their ability to peer through heavy extinction while also achieving very high spatial resolution. As a result, similar sizes are difficult to obtain at other wavelengths. Here, we assume that the energetically dominant region traced by the radio data is the energetically dominant region at other wavelengths. This allows the calculation of intensive (per unit area or volume) quantities.

Our method to do this, in general, is to assume that half of the flux density at some other wavelength of interest is enclosed in the $33 \mathrm{GHz}$ half-light area, $A_{50, \mathrm{~d}}$. We then calculate the surface brightness and related parameters (surface density, volume density) implied by this assumption. Note that in several cases, we expect optical depth to play a key role (e.g., at $1.4 \mathrm{GHz}$ or in the IR). In this case, the $\tau \approx 1$ photosphere may lie outside our calculated size (e.g., see Barcos-Muñoz et al. 2015). We discuss these cases in the individual sections and report the derived values in Table 3.5.

To take advantage of our resolution, and due to the low signal to noise of their detection, we only derive intensive quantities for the brightest components in UGC 04881 and VV 250, while for NGC 3690 we consider the system as one single source and use the addition of $A_{50, d}$ of each component as the characteristic size of the system. Note that $\mathrm{L}_{\mathrm{IR}}$ is an integrated quantity. To account for the contribution of 

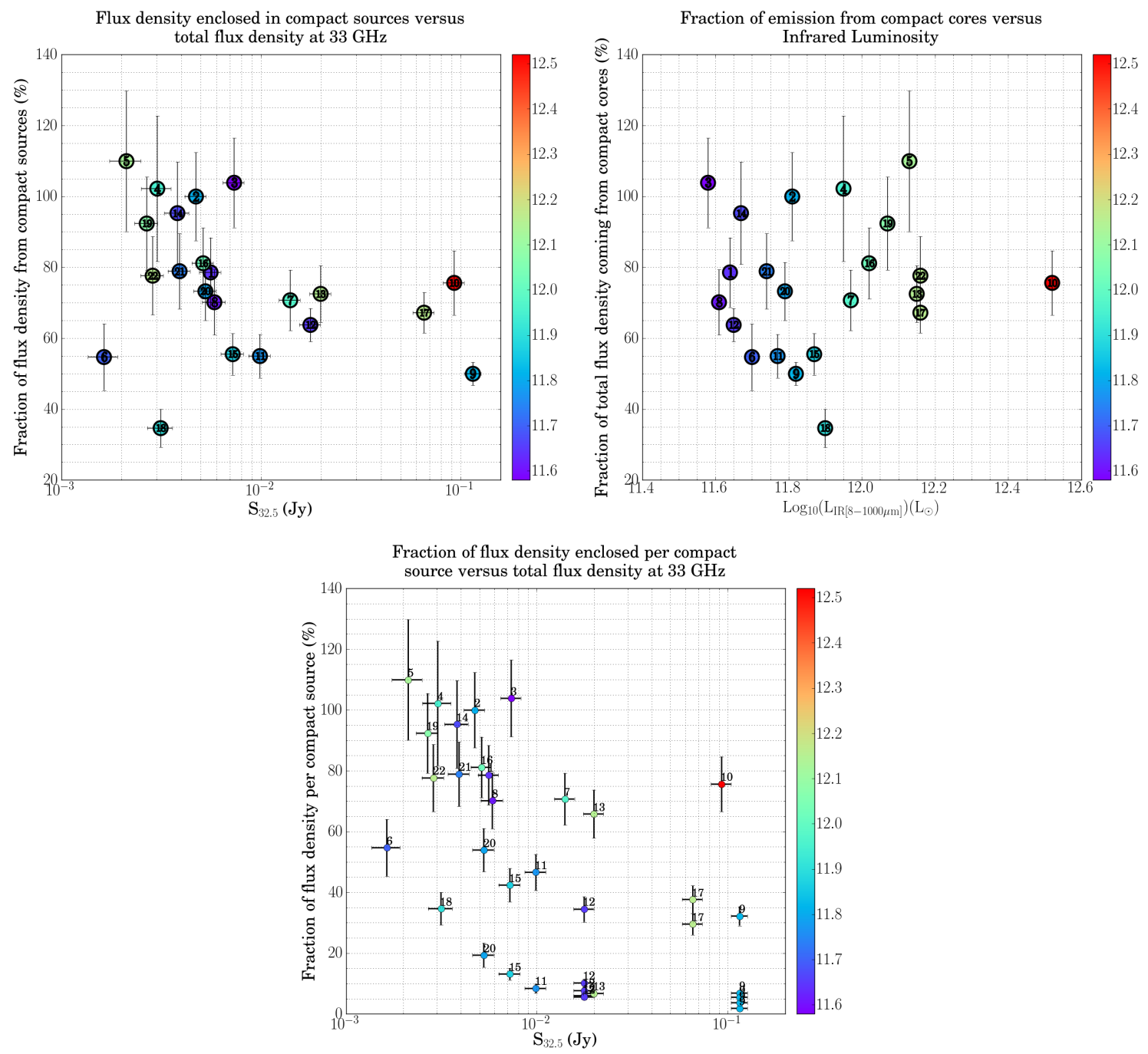

Fig. 3.4.- : Flux density contribution from compact sources. The galaxies are labeled by their Id. number and color coded by their infrared luminosity (color bar), both shown in Table 3.1. (Top left) Percentage of total flux density at $32.5 \mathrm{GHz}$ coming from compact sources as a function of the total flux density at $32.5 \mathrm{GHz}$. (Top right) Percentage of total flux density at $32.5 \mathrm{GHz}$ coming from compact sources as a function of the total infrared luminosity. (Bottom) Same as in Top left panel, but for each individual source. This plot is especially different for galaxies like NGC 3690 (\#9) and UGC 08387 (\#12) where there is more than one compact source contributing to the total flux density. Most of the $32.5 \mathrm{GHz}$ emission of our sample is concentrated in compact sources instead of extended emission. The median total compact sources contribution is $78 \%$. The rest of the emission is coming from a more extended component. This is especially evident for the system with the lower percentage in the Top panels, IRAS 17132+5313 (\#18), where a significant fraction of the total $32.5 \mathrm{GHz}$ flux density is coming from the north-east component which shows a more extended morphology (see contour map in Figure 3.1).

the brightest sources to the integrated luminosity, we assume it is distributed in the same way as the radio continuum emission, i.e., we use the contribution observed at $33 \mathrm{GHz}$ to the integrated flux density as the contribution at infrared wavelengths. This is a safe assumption considering the $33 \mathrm{GHz}$ emission follows the IR emission 
(see Section 3.5.1 and Figure 3.6), however it fails if the system is dominated by an AGN (which do not follow the radio-IR correlation). For example, for UGC 04881 the brightest component contributes $55 \%$ to the integrated flux density at $33 \mathrm{GHz}$, then we assume $55 \%$ of the total $\mathrm{L}_{\mathrm{IR}}$ is coming from the brightest component (UGC 04881NE). For VV250 this number changes to $86 \%$ for VV250a.

\subsubsection{Brightness Temperatures}

For a resolved or nearly resolved source, where beam filling is a small consideration, the brightness temperature, $T_{b}$, offers the prospect to constrain the emission mechanism and opacity of the source (e.g., Condon et al. 1991b). At radio frequencies, the brightness temperature, $\mathrm{T}_{\mathrm{b}}$, follows the Rayleigh-Jeans approximation where

$$
T_{b}=\left(\frac{S_{\nu}}{\Omega_{\text {source }}}\right) \frac{c^{2}}{2 k_{B} \nu^{2}},
$$

with $S_{\nu}$ the flux density at frequency $\nu$ and $\Omega_{\text {source }}$ the area of the source.

We (mostly) resolve our sources, allowing us to derive the average nuclear $\mathrm{T}_{\mathrm{b}}$ at $32.5 \mathrm{GHz}$ using $\Omega_{\text {source }}=\mathrm{A}_{50 \mathrm{~d}}$ and $\mathrm{S}_{\nu}=0.5 \times \mathrm{S}_{32.5}$ (see above for the explanation of the aperture correction). We also calculate $\mathrm{T}_{\mathrm{b}}$ from the point of highest intensity in the highest resolution image for each target, peak $\mathrm{T}_{\mathrm{b}}$. Figure 3.5 shows histograms of the peak and average $\mathrm{T}_{\mathrm{b}}$ at $\nu=32.5 \mathrm{GHz}$ in our targets.

The average $T_{\mathrm{b}}$ for our targets is typically a few $10 \mathrm{~s}$ to a few times $100 \mathrm{~K}$, reaching up to a few thousand in the brightest targets. In no case, do we find $\mathrm{T}_{\mathrm{b}}$ to exceed $10^{4} \mathrm{~K}$, the expected electron temperature of ionized gas. This suggest that the $\nu=$ $32.5 \mathrm{GHz}$ emission is either optically thin or optically thick at scales much smaller than the beam size. Since we begin to resolve our targets, low opacity at $32.5 \mathrm{GHz}$ appears to be the natural explanation, and is the one we adopt.

The flux densities of many of our targets have been measured at $1.4 \mathrm{GHz}$ (Table 3.3 ), but even in its most extended configuration, the VLA reaches only $\approx 1^{\prime \prime}$ resolution at this frequency. Assuming that our $32.5 \mathrm{GHz}$ sizes also describe the true extent of the $1.4 \mathrm{GHz}$ emission, we derive average $\mathrm{T}_{\mathrm{b}}$ at $1.4 \mathrm{GHz}$ that span from $10^{3}$ up to $10^{7} \mathrm{~K}$. These are considerably high values and for those systems showing $\mathrm{T}_{\mathrm{b}}>10^{4}$ 


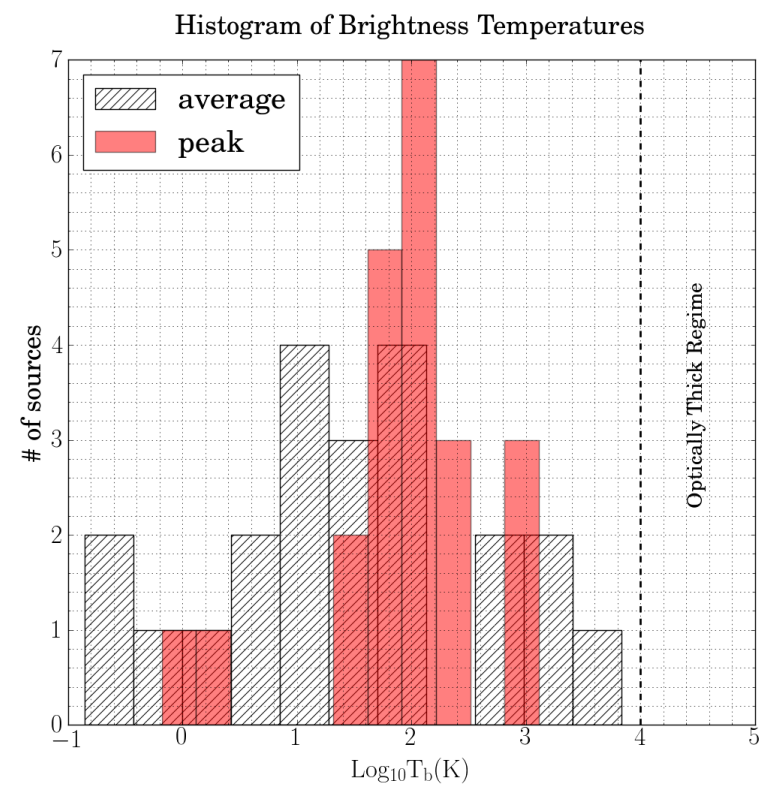

Fig. 3.5.- : Histogram of average $T_{b}$, within $A_{50}$, and peak $T_{b}$ (for details, see Section 3.4.1). The dashed vertical line indicates the limit at which a source becomes optically thick $\left(\mathrm{T}_{\mathrm{b}}=10^{4} \mathrm{~K}\right)$. Our results show that $\mathrm{T}_{b}$ is below the optically thick limit which indicates we are most likely in an optically thin regime and looking into the star forming regions.

$\mathrm{K}$, they imply that the emission at $1.49 \mathrm{GHz}$ is optically thick. Aside from that, using equation 9 from Condon et al. (1991b) we derive a maximum $\mathrm{T}_{\mathrm{b}}$ for a starburst of $10^{4.9} \mathrm{~K}$ at $1.49 \mathrm{GHz}$. At least 12 sources in our sample show derived $\mathrm{T}_{\mathrm{b}}>10^{4.9}$ K, which would imply they are powered by an AGN. We know one of those sources, Mrk 231, is dominated by an AGN, which explains why it has the highest predicted $\mathrm{T}_{\mathrm{b}}$ at $1.49 \mathrm{GHz}$. In fact, most of its emission is unresolved at $1.49 \mathrm{GHz}$. However, other sources show resolved emission at $1.49 \mathrm{GHz}$ and then the high predicted $\mathrm{T}_{\mathrm{b}}$ only imply that using the $33 \mathrm{GHz}$ is not appropriate since they are mostly affected by opacity effects. This means their emission is actually coming from an optically thick photosphere surrounding the nuclear regions (and thus are physically expanded relative to the optically-thin case). For those sources that are barely resolved even at 8.44 GHz (see maps in Condon et al. 1991b), i.e., IRAS 08572+3915, IRAS 17132+5313, IRAS $15250+3609$ and III Zw 035, extra information is needed to determine whether they are powered by an AGN and/or starbursts. 


\subsubsection{Star Formation Rate and IR Surface Density}

Infrared luminosity, $\mathrm{L}_{\mathrm{IR}}[8-1000 \mu m]$, and radio emission both trace recent star formation. IR luminosity reflects reprocessed light from young stars, while the $33 \mathrm{GHz}$ continuum captures a mix of synchrotron and thermal emission, both of which originate indirectly from young stars.

Considering a mix of synchrotron and thermal emission, Murphy et al. (2012) relate the recent star formation rate to the $33 \mathrm{GHz}$ luminosity, $L_{33 \mathrm{GHz}}$, via

$$
\begin{aligned}
\left(\frac{\mathrm{SFR}_{\nu}}{M_{\odot} \mathrm{yr}^{-1}}\right) & =10^{-27}\left[2.18\left(\frac{T_{\mathrm{e}}}{10^{4} \mathrm{~K}}\right)^{0.45}\left(\frac{\nu}{\mathrm{GHz}}\right)^{-0.1}+\right. \\
& \left.15.1\left(\frac{\nu}{\mathrm{GHz}}\right)^{-\alpha^{\mathrm{NT}}}\right]^{-1}\left(\frac{L_{\nu}}{\operatorname{erg~s}^{-1} \mathrm{~Hz}^{-1}}\right) .
\end{aligned}
$$

where $T_{e}$ is the electron temperature and $\alpha_{\mathrm{NT}}$ is the nonthermal spectral index. Murphy et al. (2012) relate the infrared luminosity to the recent star formation rate via

$$
\left(\frac{\mathrm{SFR}}{\mathrm{M}_{\odot} \mathrm{yr}^{-1}}\right)=3.15 \times 10^{-44}\left(\frac{\mathrm{L}_{\mathrm{IR}}[8-1000 \mu \mathrm{m}]}{\operatorname{ergs~s}^{-1}}\right) .
$$

In Figure 3.6 we compare IR-based and $33 \mathrm{GHz}$ based SFRs estimated for each of our targets. Following Murphy et al. (2012), we adopt $\mathrm{T}_{\mathrm{e}}=10^{4} \mathrm{~K}$ and $\alpha_{\mathrm{NT}}=0.8$ at $\nu=32.5 \mathrm{GHz}$, but note both as a source of uncertainty.

Figure 3.6 shows that these two simple SFR estimates agree in our sample. The strong outlier, source \#10, is Mrk 231, a system known to be dominated by an AGN (e.g., Ulvestad et al. 1999; Lonsdale et al. 2003) that appears to contribute substantially to the $33 \mathrm{GHz}$ emission. The other sources are consistent, at least to first order, with a simple radio-infrared correlation with normalization consistent with the Murphy et al. (2012) relations.

Assuming that our $33 \mathrm{GHz}$ size, $\mathrm{A}_{50, \mathrm{~d}}$, reflects the distribution of star formation, we can derive a star formation rate surface density, $\Sigma_{\mathrm{SFR}}$. As above, we take $\Sigma_{\mathrm{SFR}}=0.5 \times \mathrm{SFR} / \mathrm{A}_{50, \mathrm{~d}}{ }^{4}$.

\footnotetext{
${ }^{4}$ In order to obtain values that are comparable to those in the literature, we use $\mathrm{SFR}=\mathrm{SFR}_{\mathrm{IR}}$ to derive $\Sigma_{S F R}$.
} 

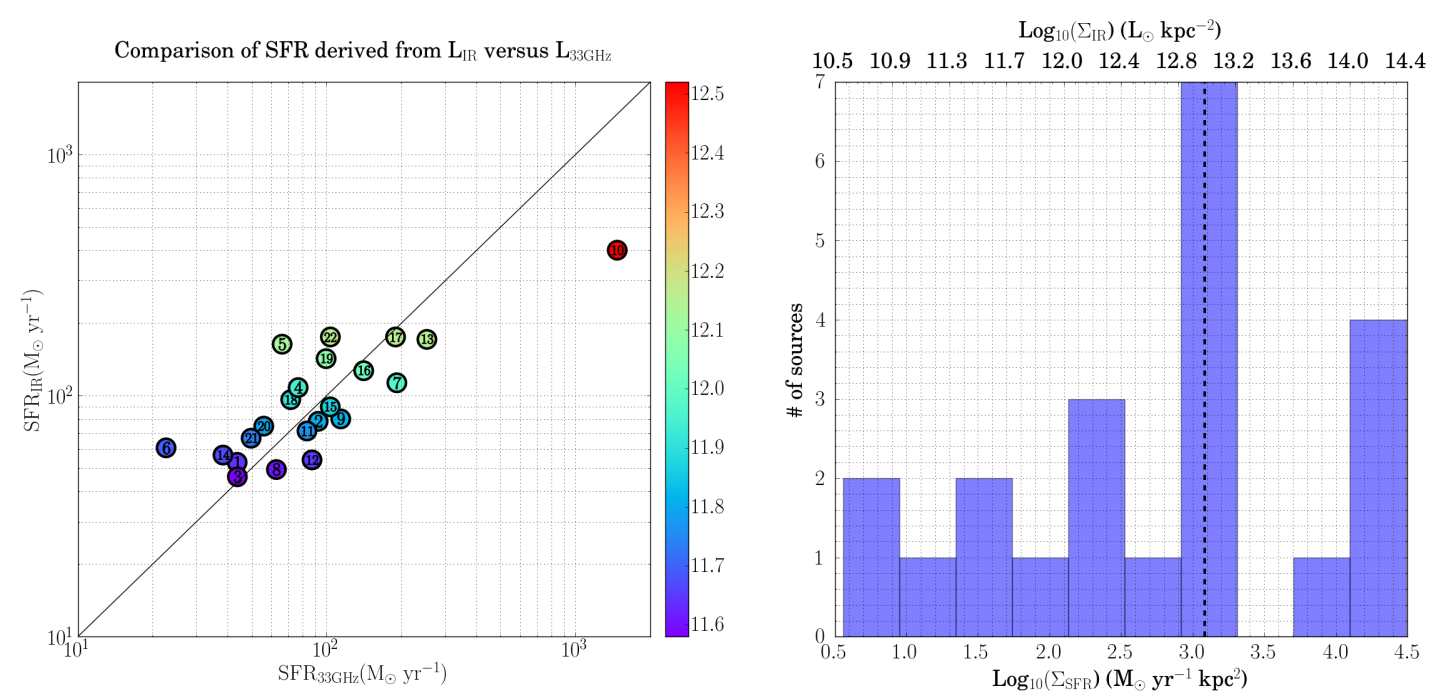

Fig. 3.6.- ( Left) SFR calculated from the IR (see equation 3.3) versus SFR calculated from the $33 \mathrm{GHz}$ luminosity (see equation 3.6). The galaxies are labeled by their Id. number and color coded by their infrared luminosity (color bar). There is an overall agreement of the SFR derived from $33 \mathrm{GHz}$ and IR, indicating the systems follow the radio-IR correlation. This is not completely true for source \#10 (Mrk 231), which is a known AGN. (Right) Histogram of $\Sigma_{I R}$ and the corresponding $\Sigma_{\mathrm{SFR}}$, both calculated assuming half of the infrared luminosity is produced within $\mathrm{A}_{50, d}$. The dashed line indicates $\Sigma_{\mathrm{IR}}=10^{13} \mathrm{~L}_{\odot} \mathrm{kpc}^{-2}$, which correspond to the dusty Eddington limit set by radiation pressure on dust (see Section 3.5.5). The range in $\Sigma$ spans about 4 orders of magnitude, which tells us our sample is somewhat diverse, despite selection biases towards the most extreme systems in the local universe. Additionally, 13 out of 22 systems have $\Sigma_{\mathrm{SFR}}>10^{2.5} \mathrm{M}_{\odot} \mathrm{yr}^{-1} \mathrm{kpc}^{2}$, which describes well the extreme nature of these systems. Those 13 systems have 2-100 times higher $\Sigma$ compared to the Orion core.

The right panel in Figure 3.6 shows our calculated values of $\Sigma_{\mathrm{SFR}}$. These span from $10^{0.6}$ up to $10^{4.5} \mathrm{M}_{\odot} \mathrm{yr}^{-1} \mathrm{kpc}^{-2}$ (right panel, bottom axis, in Figure 3.6). These are high values. The low end of this range slightly overlaps the high end of $\Sigma_{\mathrm{SFR}}$ found for $\sim \mathrm{kpc}$-sized regions of nearby disk galaxies (Leroy et al. 2012, 2013). The high end represents the highest $\Sigma_{\mathrm{SFR}}$ found for any galaxy in the local universe. The wide range also indicates diverse conditions. Even though we have observed the brightest and closest ULIRGs, these span $\sim$ four orders of magnitude in $\Sigma_{\mathrm{SFR}}$.

The IR surface brightness is also of interest. In local U/LIRGs, most of the bolometric luminosity is generated in the $8-1000 \mu \mathrm{m}$ range. By assuming that half of $\mathrm{L}_{\mathrm{IR}}$ is concentrated within $\mathrm{A}_{50, d}$, we estimate $\Sigma_{\mathrm{IR}}$ for this inner region. As is evident from Equation 3.3, $\Sigma_{\mathrm{IR}}$ is identical to $\Sigma_{\mathrm{SFR}}$ within a constant factor. Therefore we show the $\Sigma_{\mathrm{IR}}$ axis along the top of the right panel of Figure 3.6.

Our targets show $\Sigma_{\mathrm{IR}}$ from $10^{10.5}$ to $10^{14.4} \mathrm{~L}_{\odot} \mathrm{kpc}^{-2}$. The high end of this range is of particular interest. The dashed vertical line in Figure 3.6 indicates $\Sigma_{\mathrm{IR}}=10^{13} \mathrm{~L}_{\odot} \mathrm{kpc}^{-2}$. 
This value of $\Sigma_{\mathrm{IR}}$ has been argued to correspond to the Eddington limit set by radiation pressure on dust in self-regulated, optically thick disks (Thompson et al. 2005). Some sources in our sample show $\Sigma_{\mathrm{IR}} \geq 10^{13} \mathrm{~L}_{\odot} \mathrm{kpc}^{-2}$, indicating they may be maximal starbursts (see Section 3.5.5 for further discussion).

\subsubsection{Gas Surface and Column Density}

Our sample consists of gas-rich mergers. In these systems, large masses of gas are funneled to the center, where they become mostly molecular. The surface and volume densities of this gas relate closely to its self-gravity and ability to form stars. Again, we assume that our $33 \mathrm{GHz}$ size is characteristic of the system and by combining this with half of the integrated CO measurements, we estimate these quantities for our sample.

Both the assumption of the $33 \mathrm{GHz}$ characteristic size and the conversion between CO luminosity and mass introduce uncertainties into the calculation. If the densest, most concentrated gas regions are the sites where stars form most rapidly, then the $33 \mathrm{GHz}$ emission may be biased towards these regions. The mass-to-light ratio associated with CO in starbursts has also been a topic of much research (see review in Bolatto et al. 2013). We adopt a standard "starburst" value, $\alpha_{\mathrm{CO}}=0.8 \mathrm{M}_{\odot}\left(\mathrm{K} \mathrm{km} \mathrm{s}^{-1} \mathrm{pc}^{2}\right)^{-1}$ (this includes helium), but will explore the effects of a variable $\alpha_{\mathrm{CO}}$.

For a starburst $\alpha_{\mathrm{CO}}$ and coexisting gas and radio emission, we infer values for the molecular gas surface density, $\Sigma_{\text {mol }}$, from $10^{2.4}$ to $10^{5.7} \mathrm{M}_{\odot} \mathrm{pc}^{-2}$. Even the low end of this range corresponds to source-averaged surface densities in excess of many Local Group molecular clouds (e.g., Bolatto et al. 2008; Fukui \& Kawamura 2010). The high end is far in excess of the $\sim \mathrm{g} \mathrm{cm}^{-2}$ threshold commonly invoked as an immediate precondition for star formation considering dense substructure inside molecular clouds. But here this gas column density is the average across the whole energetically dominant area of a galaxy.

These values obviously depend on the mass-to-light ratio ("conversion factor") adopted to convert CO luminosity to mass. The appropriate conversion factor for starburst galaxies has been a matter of debate, with suggestions ranging from ap- 
proximately Galactic (e.g., Papadopoulos et al. 2012; Scoville et al. 2014) to low (e.g., Downes \& Solomon 1998) and highly environment-dependent (Shetty et al. 2011) values. To see the effect of a higher, Milky Way, $\alpha_{\mathrm{CO}}$ one should multiply our nominal values by $\approx 5.4$.

One class of models considers the mass surface density a main driver of the conversion factor, largely via its effect on the line width (e.g., Shetty et al. 2011; Narayanan et al. 2012). The measured sizes give us the opportunity to illustrate the effect of such a dependence on derived surface densities. To do this, we use the prescription in Bolatto et al. (2013, their equation 31) which follows Shetty et al. (2011). Neglecting any metallicity dependence and considering only the regime where $\Sigma>100 \mathrm{M}_{\odot} \mathrm{pc}^{-2}$, their prescription is

$$
\left(\frac{\alpha_{\mathrm{CO}}}{\frac{\mathrm{M}_{\odot}}{\left[\mathrm{K} \mathrm{km} \mathrm{s}^{-1} \mathrm{pc}^{2}\right]}}\right) \approx 4.35\left(\frac{\Sigma_{\text {total }}}{100 \mathrm{M}_{\odot} \mathrm{pc}^{-2}}\right)^{-0.5},
$$

where $\Sigma_{\text {total }}$ is the total mass surface density driving the potential well. We will assume our systems to be gas-dominated and take $\Sigma_{\text {total }} \sim \Sigma_{\text {gas }}$. The gas mass is the addition of the atomic (HI) gas mass and the molecular gas mass $\left(\mathrm{H}_{2}\right)$. In the central regions of U/LIRGs the HI content is often negligible, and we will consider $\Sigma_{\text {gas }} \sim \Sigma_{\text {mol }}$. We calculate the conversion factor from equation 3.4 iteratively, because $\Sigma_{\text {mol }}$ changes as $\alpha_{\mathrm{CO}}$ changes. Numerically iterating, we reach a value of $\alpha_{\mathrm{CO}}$ that converges to within $0.1 \%$. We report the gas properties derived using this surfacedensity dependent $\alpha_{\mathrm{CO}}$ in brackets, along with $\alpha_{\mathrm{CO}}$ for each source, in Table 3.5. The effect of applying this correction is to narrow the range of derived gas surface densities, as the high surface density systems have high $\alpha_{\mathrm{CO}}$.

The gas surface density values derived here translate to average, Hydrogen column densities in the nuclear regions that range from $10^{22.5} \mathrm{~cm}^{-2}$ to $10^{25.8} \mathrm{~cm}^{-2}$ when using $\alpha_{\mathrm{CO}}=0.8$ and $10^{22.9} \mathrm{~cm}^{-2}$ to $10^{25} \mathrm{~cm}^{-2}$ when using the surface-density dependent conversion factor. Assuming a Galactic dust-to-gas ratio (Bohlin et al. 1978), which may be roughly appropriate (Rupke et al. 2008; Iono et al. 2009), these column densities imply line of sight extinctions of $A_{V} \sim 17$ to $3 \times 10^{4}$ mag, for a starburst 
conversion factor, and $\sim 40$ to $6 \times 10^{3}$ mag, for a surface-density dependent conversion factor, through the systems.

\subsubsection{Gas Volume Density}

We can also derive properties within a volume, instead of a surface. This requires additional assumptions for the geometry of the star formation distribution. We consider the most basic approach and assume that our sources are three dimensional Gaussians. In this case, $\sim 30 \%$ of the mass exists inside the FWHM of the Gaussian ${ }^{5}$, $\mathrm{R}_{50, d}$.

This geometry implies $\mathrm{n}_{\mathrm{H}_{2}}$ from $10^{0.5} \mathrm{~cm}^{-3}$ to $10^{5.5} \mathrm{~cm}^{-3}$ by using a fixed conversion factor of 0.8 and from $10^{0.8} \mathrm{~cm}^{-3}$ to $10^{4.7} \mathrm{~cm}^{-3}$ by using the conversion factor that varies for each source. The free fall collapse time associated with these densities ranges from $0.1-30 \mathrm{Myr}$, and from $0.2-20 \mathrm{Myr}$.

\subsection{Discussion}

In Figure 3.1 we show the $33 \mathrm{GHz}$ continuum contour maps from which we measured half light areas with equivalent radii that go from $\sim 20$ pc up to $\sim 1.7 \mathrm{kpc}$. Due to the nature of this survey, high resolution combined with sensitivity to different spatial scales, these sizes represent the best measurements to date of the energetically dominant regions in the brightest, closest U/LIRGs from GOALS. These sizes combined with the integrated flux density measurements allow us to study the physical properties of the nuclear regions in our sample. In the following subsections we will discuss the nature of the $33 \mathrm{GHz}$ emission and implications of the derived quantities in the previous section for star formation scaling relations, optical depth and radiation pressure feedback.

\footnotetext{
${ }^{5}$ This is the correction to obtain the mass inside a sphere of radius $\mathrm{R}_{50, d}$ (see Section 3.3 .3 ) with a Gaussian mass distribution
} 

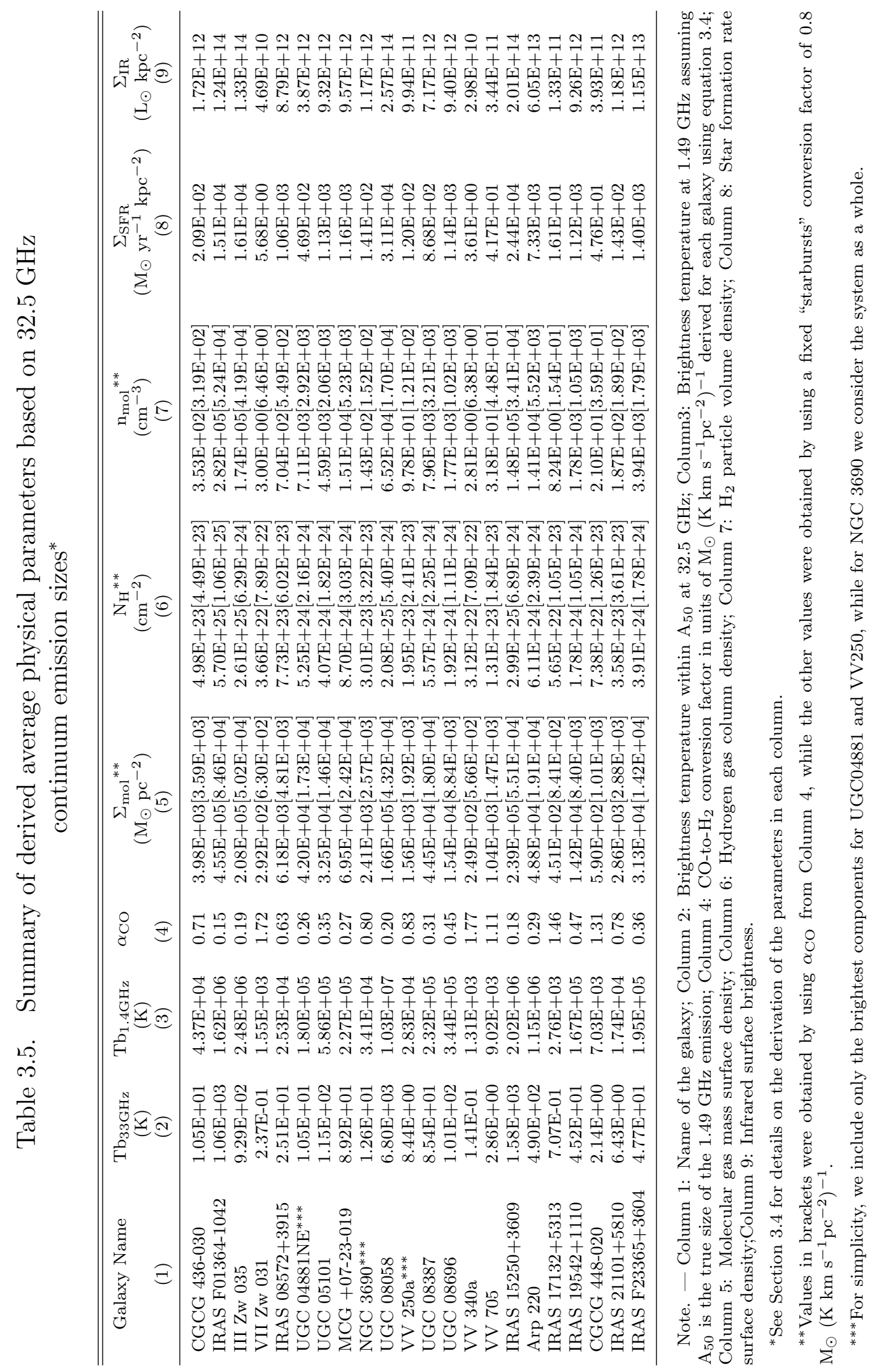


\subsubsection{Nature of the $33 \mathrm{GHz}$ Radio Emission}

In models like those of Condon (1992) and Murphy et al. (2012), the radio SED reflects a mixture of thermal and nonthermal emission. What powers the emission that we observe from U/LIRGs at $33 \mathrm{GHz}$ ? In the Condon (1992) model for a starburst galaxy like M82, about $50 \%$ of the total $33 \mathrm{GHz}$ continuum is produced by free-free ("thermal") emission; for comparison, $<10 \%$ of the emission is expected to be produced by free-free emission at $1.5 \mathrm{GHz}$.

We have several constraints on the nature of the emission mechanism in our targets: the SED shape, the brightness temperature, and the comparison with the SFR implied by the IR. Together, these do indicate some 10s of percent contribution of thermal emission to the $33 \mathrm{GHz}$ flux density, with the balance being synchrotron. However, a detailed understanding of the emission mechanism will need to wait for better coverage of the radio SED in these targets.

Brightness Temperature and Optical Depth: The brightness temperature of optically thick free-free emission is expected to be $\sim 10^{4} \mathrm{~K}$. If our $33 \mathrm{GHz} T_{b}$ exceeded this value, this would provide evidence that synchrotron dominates the emission. Figure 3.5 shows that in our data the average $\mathrm{T}_{\mathrm{b}}$ does not exceed this limit. Either the emission is patchy within our beam, or the emission at $33 \mathrm{GHz}$ is optically thin. Thus, the brightness temperature in our sources allows for a normal mix of emission mechanisms and is consistent with optically thin free-free emission making up a large part (or all) of the observed $33 \mathrm{GHz}$ flux density.

If we neglect filling factor effects and assume that $\approx 50 \%$ of the total $\mathrm{T}_{\mathrm{b}}$ is due to thermal emission, then we can estimate the optical depth of the free-free emission. We derive $\tau_{\text {thermal }} \sim \mathrm{T}_{\mathrm{b}} / \mathrm{T}_{\mathrm{e}} \leq 0.34$ for all our sample. This number is still less than 1, therefore optically thin, even if we assume $100 \%$ of the $33 \mathrm{GHz}$ flux density is due to thermal emission.

Spectral Index: For a mixture of synchrotron ("non-thermal") emission with a power-law spectral energy distribution and optically thin free-free emission, Condon \& Yin (1990) give the following approximation to the fraction of emission that is 
thermal,

$$
\frac{S}{S_{\mathrm{T}}} \sim 1+10\left(\frac{\nu}{\mathrm{GHz}}\right)^{0.1+\alpha_{\mathrm{NT}}},
$$

where $\mathrm{S}$ is the total flux density, $\mathrm{S}_{\mathrm{T}}$ is the flux density from thermal emission, and $\alpha_{\mathrm{NT}}$ is the typical non-thermal spectral index $\sim-0.8$.

If we use equation 3.5 and $\mathrm{S}_{5.95}$ from Table 3.3, and knowing that $\mathrm{S}_{\mathrm{T}} \propto \nu^{-0.1}$, we predict an integrated $\alpha_{6-33}$ of -0.53 . This number approaches -0.8 as the thermal fraction decreases and non-thermal emission starts to dominate. From Figure 3.2, we observe that 17 out of the 22 systems in our sample show $\alpha_{6-33}<-0.53$. This implies that non-thermal emission dominates most of our targets. This is in agreement with what we found for Arp 220 using both 6 and $33 \mathrm{GHz}$ in Barcos-Muñoz et al. (2015). However, note that changing our assumed $\alpha_{\mathrm{NT}}$ could change this conclusion and that $\alpha_{\mathrm{NT}}$ over the range $6-33 \mathrm{GHz}$ is not strongly constrained for our targets. Multi-frequency observations, particularly at high frequency, suggest curvature in the radio SED (e.g., see Clemens et al. 2008, 2010; Leroy et al. 2011; Marvil et al. 2015) so that the power-law assumption for the non thermal emission model in Equation 3.5 represents a simplification. Observations that cover a wide band (e.g., using the current $\sim 8 \mathrm{GHz}$ VLA bandwidth) will allow for a more complex treatment.

Spectral Index and Implied Opacity at Lower Frequencies: By following the same approach, we predict an integrated $\alpha_{1.5-6}$ of -0.71 . However, less than half of the sample show spectral indices that agree with the predicted value. Most of the systems show shallower spectral indices, which is most likely due to opacity affecting the low frequency emission, especially the observations at $1.49 \mathrm{GHz}$ where free-free absorption is known to play a major role in compact starbursts (see Condon et al. 1991b). In fact, in Figure 3.2 we also observe a change in slope as frequency increases for several sources, from shallower to steeper in most cases (Mrk 231 even shows a change from positive $\alpha_{1.5-6}$ to a negative $\alpha_{6-33}$ indicating $\tau_{\text {thermal }}$ becomes one at some frequency between 1.5 and $\left.33 \mathrm{GHz}^{6}\right)$.

Several systems show the opposite trend, exhibiting steep $\alpha_{1.5-6}$ and a shallower $\alpha_{6-33}$. These may have a higher thermal fraction than the other targets or may

\footnotetext{
${ }^{6}$ This turnover frequency normally occurs at $\mathrm{MHz}$ frequencies, when present, and it increases for high star forming, very compact systems.
} 
have some other source of contribution to the $33 \mathrm{GHz}$ emission, e.g., anomalous dust emission (Draine \& Lazarian 1998; Ali-Haïmoud et al. 2009; Murphy et al. 2010). Again, more detailed SED coverage could confirm this interpretation. Another possible explanation includes contribution from thermal dust (normally being important at higher frequencies $\sim 100 \mathrm{GHz}$ ). The simplest explanation, however, remains a higher balance of thermal relative to non-thermal emission.

In Figure 3.2, we find a tentative correlation between $\alpha_{1.5-6 \mathrm{GHz}}$ and $\mathrm{A}_{50, \mathrm{~d}}$ with a shallower spectral index for more compact sources. This is the expected trend since more compact sources are also more opaque, with $1.5 \mathrm{GHz}$ emission suppressed due to a higher opacity relative to $6 \mathrm{GHz}$.

Integrated spectral indices only give us a partial view of the processes that are powering star formation in our sample. We require more detailed spectral index maps to dissect the distribution of the radio emission. We will report resolved spectral index maps between 6 and $33 \mathrm{GHz}$ in a future paper (Barcos-Munõz et al. in prep).

Expectations from IR-Based SFRs: The contrast of the $33 \mathrm{GHz}$ flux density with the total IR emission also sheds some light on the emission mechanism. Inasmuch as the IR tells us about the star formation rate, it also makes a prediction for the expected thermal emission, along with some simplifying assumptions.

We derive the expected free-free emission, $\mathrm{S}_{\mathrm{T}}$, and then thermal fraction $\mathrm{S}_{\mathrm{T}} / \mathrm{S}$, at $33 \mathrm{GHz}$ by assuming that all the IR luminosity is due to star formation and that no ionizing photons are absorbed by dust. We use equation 3.3 and the thermal SFR from Table 8 in Murphy et al. (2012), which relates SFR and the thermal luminosity, $\mathrm{L}^{\mathrm{T}}$, by the following equation,

$$
\begin{aligned}
\left(\frac{\mathrm{SFR}_{\nu}^{\mathrm{T}}}{M_{\odot} \mathrm{yr}^{-1}}\right) & =4.6 \times 10^{-28} \\
& \left(\frac{T_{\mathrm{e}}}{10^{4} \mathrm{~K}}\right)^{-0.45}\left(\frac{\nu}{\mathrm{GHz}}\right)^{0.1}\left(\frac{L_{\nu}^{\mathrm{T}}}{\operatorname{erg~s}^{-1} \mathrm{~Hz}^{-1}}\right)
\end{aligned}
$$

In this way, we predict the thermal radio emission expected given the IR luminosity. Comparing it to the measured $33 \mathrm{GHz}$ flux density, we derive the thermal fractions shown in the top-right panel in Figure 3.2. 
From our analysis of the spectral index, we expect thermal fractions $\leq 50 \%$. Figure 3.2 shows that based on the prediction from the IR, most of our sources have thermal fractions $\approx 50-100 \%$. We expect that this is the combination of two effects. First, even if the IR is all powered by star formation, some of the ionizing photons produced by young stars will be absorbed by dust and thus do not produce free-free emission. These should not be counted in our prediction for the thermal emission, and the true thermal fraction would be accordingly smaller. We highlighted a similar situation in Arp 220, where the predicted thermal fraction is $\sim 50 \%$ but SED analysis shows it should be closer to 35\% (see Barcos-Muñoz et al. 2015). Second, as noted above, the SED-based estimates remain hampered by the lack of good, wide-band coverage of the spectral energy distribution. As long as the adopted non-thermal spectral index (or SED) remains uncertain, so do the thermal fractions estimated in this way.

Two sources, UGC 04881 and IRAS $08572+3915$ show thermal fractions $>100 \%$, meaning very high ratios of IR to radio emission (see Figures 3.6 and 3.7). This IR excess has been reported before for IRAS 08572+3915 (see discussion in Yun et al. 2004), and this system was already noted as an interesting source in discussion of first results from our survey (Leroy et al. 2011).

Radio-IR Correlation at $33 \mathrm{GHz}$ : As a more observational restatement of the previous result, we derive $q_{33}$, the ratio of IR flux density to radio flux density at $33 \mathrm{GHz}:$

$$
q_{\nu}=\log _{10}\left(\left(S_{F I R} / 3.75 \times 10^{12} H z\right) / S_{\nu}\right) .
$$

Here, $S_{\nu}$ is the flux density at frequency $\nu$ in units of $\mathrm{W} \mathrm{m}^{-2} \mathrm{~Hz}^{-1}$, and $\mathrm{S}_{\mathrm{FIR}}=$ $1.26 \times 10^{-14}\left(2.58 \mathrm{~S}_{60 \mu \mathrm{m}}+\mathrm{S}_{100 \mu \mathrm{m}}\right)$, in units of $\mathrm{W} \mathrm{m}^{-2}$, is the far-infrared flux density, with the flux density at 60 and $100 \mu \mathrm{m}$ measured in Jy.

We plot our calculated $q_{33}$ in Figure 3.7. We find a median $q_{33} \approx 3.32$ and a dispersion of 0.19 dex. Our $q_{33}$ is similar to that found by Rabidoux et al. (2014) studying regions in local star forming galaxies, but we find a tighter correlation. Their measured dispersion is 0.1 dex larger than ours. In fact, the 0.19 dex in dispersion we observed for $\mathrm{q}_{33}$ is similar to that found in Condon et al. (1991b) at $1.49 \mathrm{GHz}$. 


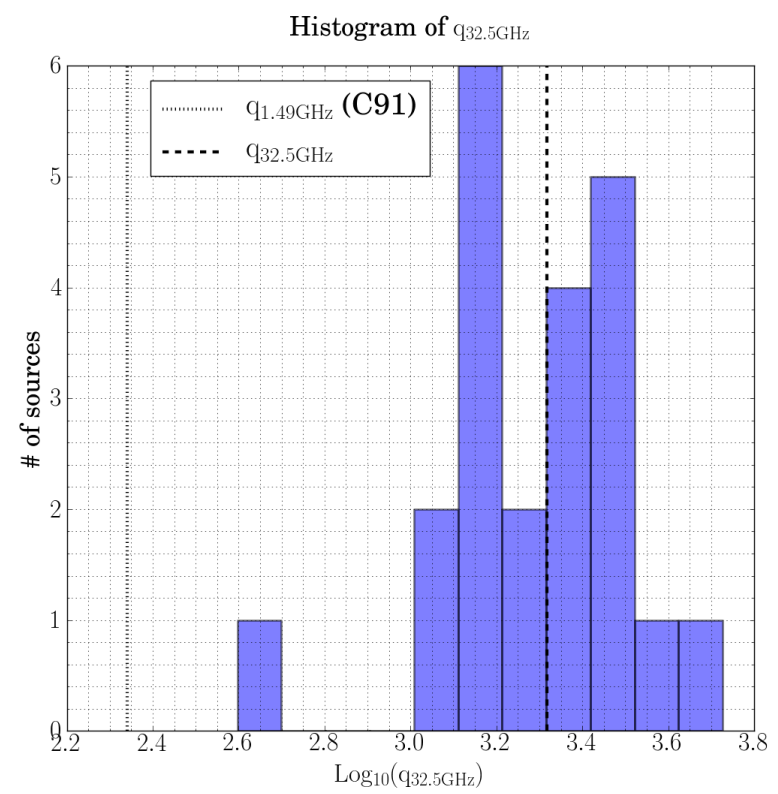

Fig. 3.7.- : Histogram of $\mathrm{q}_{32.5 \mathrm{GHz}}$ obtained using equation 3.7. The dashed line shows the median value for $\mathrm{q}_{32.5 \mathrm{GHz}}$ at 3.3. For comparison, we also show the median value for $\mathrm{q}_{1.49 \mathrm{GHz}}$ at 2.34 from Condon et al. (1991b). The most deviant source in the histogram is Mrk 231 ( $\mathrm{q} \sim 2.37$ ), which is a known AGN and does not follow the radio-FIR correlation (see Figure 3.6).

\subsubsection{Physical Conditions at the Heart of Local Major Merg- ers}

Our estimates for the sizes of the energetically dominant regions in our sample imply that a large part of the star forming activity, and so presumably also the gas that fuels it, is concentrated in areas with equivalent radii that go from $20 \mathrm{pc}$ up to 1.7 $\mathrm{kpc}^{7}$. Applying these sizes to global quantities using the proper aperture corrections, we estimate $\Sigma_{\mathrm{SFR}}, \Sigma_{\mathrm{IR}}, \mathrm{N}_{\mathrm{H}}$, and $\mathrm{n}_{\mathrm{mol}}$ for our targets.

The resulting values span a wide range, typically 4 dex. The high end of the range for each property is among the highest average gas, SFR, or luminosity surface density measured for any galaxy. The low end of the range is still high compared to values found in "normal" disk galaxies: our lowest density systems have $\Sigma_{\text {mol }} \sim 10^{2}-$ $10^{3} \mathrm{M}_{\odot} \mathrm{pc}^{-2}$ and $\Sigma_{\mathrm{SFR}} \sim 10^{0}-10^{1} \mathrm{M}_{\odot} \mathrm{yr}^{-1} \mathrm{kpc}^{-2}$. These already resemble the highest

\footnotetext{
${ }^{7}$ This is without including the upper limits obtained for the faint components in the systems UGC 04881 and VV250, for which we did not derive the physical parameters described in section 3.4 .
} 
kpc-resolution values (which come from active galaxy centers) found in (Leroy et al. 2013) (see bottom panel in Figure 3.7). More, the gas surface densities in our sample, even the lowest values, resemble those found for individual molecular clouds, but here they extend over the whole energetically dominant region of our targets. This implies average interstellar gas pressures in our targets that match or exceed those found inside individual clouds. Because of this high pressure, a Milky Way GMC dropped in to any of our targets would not remain an isolated, self-gravitating object. Selfgravitating, overpressured clouds in our targets must be more extreme and denser than clouds in normal galaxies, a conjecture born out by observations in nearby starburst galaxies (e.g., Keto et al. 2005; Wei et al. 2012; Leroy et al. 2015; Johnson et al. 2015).

About half (12) of our 22 targets show galaxy-averaged $\Sigma_{\mathrm{SFR}} \geq 10^{2.7} \mathrm{M}_{\odot} \mathrm{yr}^{-1} \mathrm{kpc}^{2}$. This corresponds to $\geq 2$ times higher than the $\Sigma_{\text {SFR }}$ that would be inferred based on the IR emission from the Orion core (Soifer et al. 2000). Several (11) sources show $\Sigma_{\mathrm{SFR}}>10^{3} \mathrm{M}_{\odot} \mathrm{yr}^{-1} \mathrm{kpc}^{-2}$, corresponding to $\Sigma_{\mathrm{IR}}>10^{13} \mathrm{~L}_{\odot} \mathrm{kpc}^{-2}$. This value has been put forward as the theoretical maximum $\Sigma_{\mathrm{SFR}}$ for a radiation pressure supported optically thick disk (Scoville 2003; Thompson et al. 2005) (see Section 3.5.5 for further discussion).

The high column densities obscure the energetically dominant regions at non-radio wavelengths. Assuming a "starburst" conversion factor, 12 of our sources show hydrogen column densities consistent with being Compton-thick, $\mathrm{N}_{\mathrm{H}}>1.5 \times 10^{24} \mathrm{~cm}^{-2}$ (e.g., Comastri 2004). As mentioned above, the implied optical extinctions are extreme, $17-3 \times 10^{4} \mathrm{mag}$ for our sample for a Galactic dust-to-gas ratio. Even infrared wavelengths, at which a normal star-forming galaxy is usually optically thin, shows significant opacity. At $100 \mu \mathrm{m}$, for a mass absorption coefficient of $\kappa_{100}=31.3 \mathrm{~cm}^{2} \mathrm{~g}^{-1}$ (Li \& Draine 2001), the dust opacity of our targets is $\tau_{100} \sim 0.02-30$, with those same 12 Compton-thick sources being optically thick at $100 \mu \mathrm{m}$, i.e., $\tau_{100}>1$. 


\subsubsection{The $[\mathrm{CII}]$ deficit}

Several studies have reported a "deficit" in the [C II] $158 \mu \mathrm{m}$-to-far infrared luminosity ratio, $L_{[\mathrm{CII}]} / L_{\mathrm{FIR}}$, in U/LIRGs relative to lower luminosity star-forming galaxies (e.g., Malhotra et al. 2001; Díaz-Santos et al. 2013). The $L_{[\mathrm{CII}]} / L_{\mathrm{FIR}}$ is shown to decrease with increasing dust temperature, mid-IR measured opacity, star formation efficiency $\left(L_{\text {stars }} / M_{\mathrm{H}_{2}}\right)$ and infrared surface density (where Spitzer and Herschel data are utilized to measure sizes). Thus, the collisional energy required to produce [C II] is being suppressed in the compact, dense starburst environments of U/LIRGs, and/or the infrared luminosity is being increased. Here, we examine two aspects of the deficit using radio measurements.

First, we make use of our radio size measurements to derive infrared surface densities. In Figure 3.8 (top left panel), we plot the $L_{[\mathrm{CII}]} / L_{\mathrm{FIR}}$ as a function of the star formation rate surface density, $\mathrm{SFR}_{33 \mathrm{GHz}}$; the plot is further confirmation that $L_{[\mathrm{CII}]} / L_{\mathrm{FIR}}$ decreases as a function of increasing $\Sigma_{\mathrm{SFR}_{33 \mathrm{GH}}}$. We also show in Figure 3.8 (top right panel) $L_{[\mathrm{CII}]} / L_{\mathrm{FIR}}$ as a function of $\mathrm{A}_{50}$, where there is a clear trend of increasing compactness with decreasing $L_{[\mathrm{CII}]} / L_{\mathrm{FIR}}$. Second, we plot $L_{[\mathrm{CII}]} / L_{\mathrm{FIR}}$ as a function of the 1.5-to-6 GHz spectral index, $\alpha_{1.5-6 \mathrm{GHz}}$ (bottom panel). We find that the spectral index flattens as a function of decreasing $L_{[\mathrm{CII}]} / L_{\mathrm{FIR}}$. This flattening is due to increasing opacity (e.g., see Murphy et al. 2013) and is further confirmation that these low $L_{[\mathrm{CII}]} / L_{\mathrm{FIR}} \mathrm{U} /$ LIRGs are among the most heavily obscured systems. It is also worth noting that, with the exception of IR F08572+3915, the five U/LIRGs (Mrk 231, IRAS 15250+3608,IIIZW035, IR F01364-1042, and Arp 220) with the flattest spectral index have the lowest estimated thermal fraction at $33 \mathrm{GHz}$. Thus, these five systems have the highest opacity at radio frequencies and the lowest thermal fractions at $33 \mathrm{GHz}$. These results are broadly consistent with our detailed study of Arp 220 (Barcos-Muñoz et al. 2015), where we presented evidence of suppressed 33 $\mathrm{GHz}$ thermal emission and speculated that the suppression is due to the absorption

of ionizing UV photons by dust concentrated within the HII regions. Such scenario would also explain the lack of collisional energy available for [C II] production. 

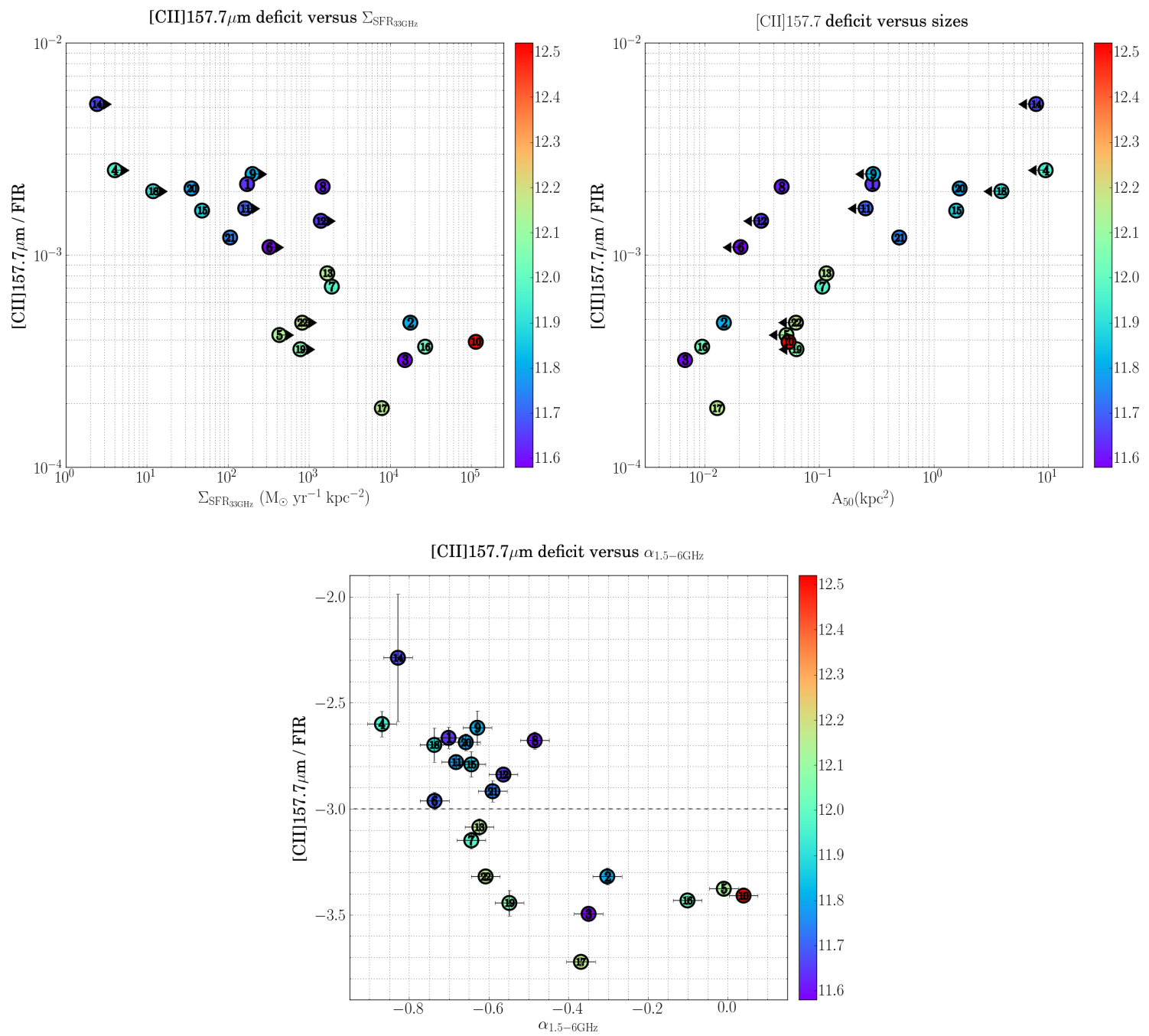

Fig. 3.8.-: (Top left) $[\mathrm{CII}] 158 \mu \mathrm{m}$ deficit as given by [CII] $158 \mu \mathrm{m} / \mathrm{FIR}$, where FIR is the far infrared flux density

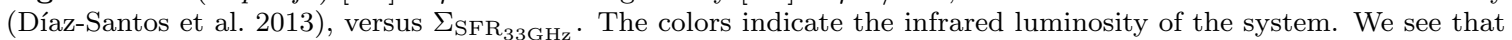
the most luminous galaxies in our sample show the highest deficit (smallest ratio of [CII] flux/FIR). (Top right) [CII] deficit as a function of half-light area. The symbols are the same as in the left panel. We observe a higher [CII] deficit for the more compact objects. (Bottom left) [CII] deficit as a function of $\alpha_{1.5-6 G H z}$. The sources with the highest deficit show the flattest spectral index between 1.5 and $6 \mathrm{GHz}$. This is consistent with the finding that the [CII] deficit is inversely proportional to mid-IR opacity measurements, i.e., low [CII] $158 \mu \mathrm{m} / \mathrm{FIR}$ sources are deeply buried.

\subsubsection{Implications for Star Formation Scaling Relations}

The observed scaling between star formation rate surface density, $\Sigma_{\mathrm{SFR}}$, and gas surface density, $\Sigma_{\text {gas }}$, is often used as a main diagnostic of the physics of star formation in galaxies (e.g., Kennicutt 1998). Kennicutt (1998) fit a scaling between galaxyaveraged $\Sigma_{\text {SFR }}$ and $\Sigma_{\text {gas }}$ that describes both normal disk galaxies and starbursts. The 
starbursts in Kennicutt (1998) have high $\Sigma_{\text {SFR }}$ and $\Sigma_{\text {gas }}$ and include U/LIRGs like those that we study here.

The contrast between the normal disks (low $\left.\Sigma_{\mathrm{SFR}}, \Sigma_{\text {gas }}\right)$ and the starburst galaxies (high $\Sigma_{\mathrm{SFR}}, \Sigma_{\text {gas }}$ ) played a main role in driving the best overall fit of Kennicutt (1998), $\Sigma_{\mathrm{SFR}} \sim \Sigma_{\text {gas }}^{1.4}$. This contrast depends on the sizes adopted for the starburst galaxies. Changing the size affects both surface densities by the same factor, but because the overall relationship between $\Sigma_{\mathrm{SFR}}$ and $\Sigma_{\text {gas }}$ is non-linear, the adopted size affects the slope.

In Figure 3.9 we place our systems in the $\Sigma_{\mathrm{SFR}}-\Sigma_{\text {gas }}\left(\right.$ or $\left.\Sigma_{\mathrm{SFR}}-\Sigma_{\mathrm{mol}}\right)$ plane (see Section 3.4.2 and 3.4.3 for details on the derivation of $\Sigma_{\mathrm{SFR}}$ and $\left.\Sigma_{\mathrm{mol}}\right)$. In the top-left panel, we show only our targets and adopt a fixed $\alpha_{\mathrm{CO}}=0.8 \mathrm{M}_{\odot} \mathrm{pc}^{-2}\left(\mathrm{~K} \mathrm{~km} \mathrm{~s}^{-1}\right)$. Our sources show high surface densities and an approximately linear relationship. A non-linear least-squares fit $^{8}$ yields

$$
\log _{10}\left(\Sigma_{\mathrm{SFR}}\right)=(1.04 \pm 0.10) \log _{10}\left(\Sigma_{\mathrm{mol}}\right)-(1.38 \pm 0.43)
$$

This slope is in good agreement to the results found by Liu et al. (2015) for disk galaxies and for U/LIRGs. Daddi et al. (2010) and Genzel et al. (2010) also noted that the internal relationship for starburst galaxies was more nearly linear than the relationship using both types of galaxies, giving rise to the idea of "two sequences" of star formation. With a slope close to unity, another way to express Equation 3.8 is that for a "starburst" conversion factor, we find a typical gas depletion time, $\tau_{\text {dep }} \equiv M_{\text {mol }} /$ SFR, of $\tau_{\text {dep }} \sim 20$ Myr for our targets.

In addition to the size, the adopted conversion factor can have a large effect on the results. Because we find an approximately linear relationship within our sample, shifting from one constant $\alpha_{\mathrm{CO}}$ to another will not significantly affect the slope. For example, if we use a Galactic $\alpha_{\mathrm{CO}}=4.35 \mathrm{M}_{\odot} \mathrm{pc}^{-2}\left(\mathrm{~K} \mathrm{~km} \mathrm{~s}^{-1}\right)$ instead, the coefficient would shift to $-2.14 \pm 0.50$, raising the depletion time to $\tau_{\text {dep }}=110$ Myr. For comparison, Leroy et al. (2013) find a significantly longer time, 1.6 Gyr, in the

\footnotetext{
${ }^{8} \mathrm{We}$ used the scipy.optimize.curve fit algorithm and a function of the form $\mathrm{Y}=$ slope $\mathrm{X}+$ coefficient to obtain the slope and coefficient, and their standard deviation errors. We excluded sources with lower limits to their sizes.
} 

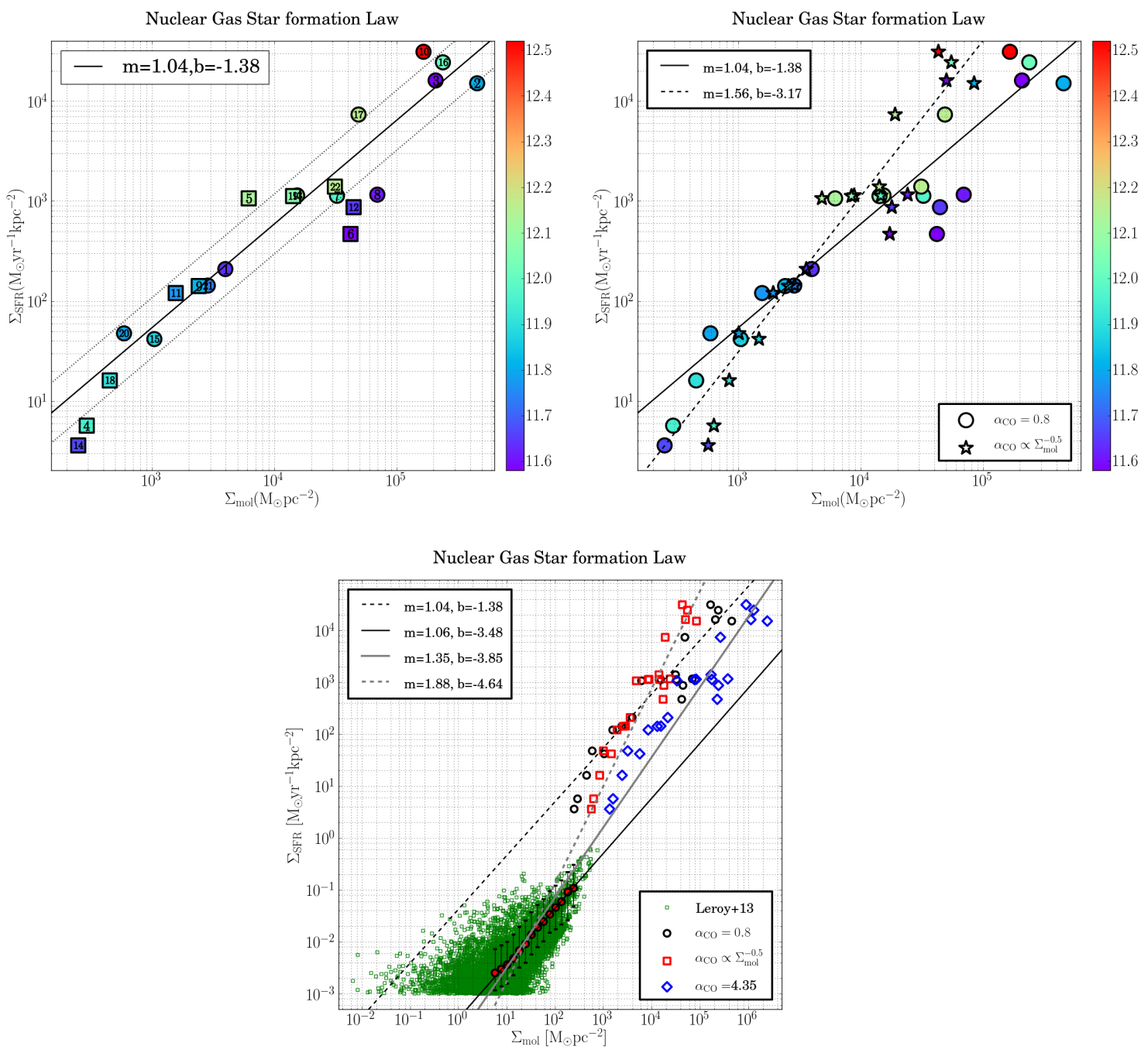

Fig. 3.9.- - Kennicutt-Schmidt (KS) law of star formation within $A_{50, d}$ (see Section 3.4 for details). The SFR was calculated based on the IR luminosity and the molecular gas mass was obtained using an $\alpha_{\mathrm{CO}}$ factor. (Top left) The mass of the gas was calculated using a fixed CO-to- $\mathrm{H}_{2}$ conversion factor of 0.8 (typical for ULIRGs). The squares represent lower limits and the solid line is the fit to the data. The dotted lines are separated by 0.3 dex from the fit and the colors represent the infrared luminosity of each system. (Top right) The circles show the values from the top left panel, i.e., with the gas obtained using a fixed conversion factor of 0.8 , and the solid line shows the fit. The stars show values where the gas was obtained using a conversion factor that varied for each source (see Table 3.5) and the dashed line is the fit to the data. (Bottom) Comparison between values from nearby disk galaxies from Leroy et al. (2013) (green squares) and from our sample using different values of $\alpha_{\mathrm{CO}}$ (other symbols). The dashed line shows the fit to our data using a conversion factor of 0.8 , and the solid line shows the fit to the binned data from Leroy et al. (2013) (red circles). The best fit to the disk galaxies and U/LIRGs (using a Galactic conversion factor, blue diamonds) is shown by the solid grey line (see Section 3.5.4 for more details). By using a conversion factor that depends on the gas surface density of the source (red squares), we obtain a steeper slope (dashed grey line) compared to that obtained using a fixed conversion factor. This indicates the crucial role that $\alpha_{\mathrm{CO}}$ plays when studying the KS law. The nuclear regions in local U/LIRGs occupy the higher end of the star formation law indicating they host more extreme environments in comparison to disk galaxies. 
disks of nearby normal galaxies.

Several suggestions posit a continuous variation in $\alpha_{\mathrm{CO}}$ that depends on surface density (see above). Adopting such prescription affects the slope of the derived relation. If we adopt the surface density-dependent slope discussed in Section 3.4.3, the best fit shifts to

$$
\log _{10}\left(\Sigma_{\mathrm{SFR}}\right)=(1.56 \pm 0.14) \log _{10}\left(\Sigma_{\mathrm{mol}}\right)-(3.17 \pm 0.59) .
$$

The top-right panel in Figure 3.9 shows our data for two cases: a fixed "starburst" conversion factor and the surface-density dependent value. Internal to the starburst sample, the linearity or non-linearity of the slope depends entirely on the treatment of the conversion factor; the apparent relationship between $\Sigma_{\text {SFR }}$ and CO luminosity surface brightness is approximately linear.

As mentioned above, the contrast between normal disk galaxies and starbursts played a large role in determining the Kennicutt (1998) fit. The bottom panel of Figure 3.9 explores this contrast. There, we compare our results to those found for kpc-size regions drawn from 30 nearby disk galaxies by Leroy et al. (2013). Individual regions appear as green squares and the median and scatter in $\Sigma_{\mathrm{SFR}}$, in bins of fixed $\Sigma_{\text {mol }}$, appear as red points with error bars. Note that, in contrast to Kennicutt (1998), we consider only the molecular gas component of the ISM, and, in the normal galaxies, we consider individual kpc-sized regions. Kennicutt (1998) include atomic gas and consider whole-disk averages. We chose our approach to focus on star-forming (molecular) gas in comparable sized regions in order to contrast the ability of gas to form stars in the two types of systems.

Figure 3.9 shows a significant contrast between disks and our starburst sample, even for matched $\alpha_{\mathrm{CO}}$ (a similar contrast was seen when comparing $\tau_{\text {dep }}$ ). In that case, $\alpha_{\mathrm{CO}}=4.35 \mathrm{M}_{\odot} \mathrm{pc}^{-2}\left(\mathrm{~K} \mathrm{~km} \mathrm{~s}^{-1}\right)$ for both samples, a fit to our sample and the Leroy et al. (2013) bins yield:

$$
\log _{10}\left(\Sigma_{\mathrm{SFR}}\right)=(1.35 \pm 0.03) \log _{10}\left(\Sigma_{\mathrm{mol}}\right)-(3.85 \pm 0.12)
$$


Meanwhile, adopting the starburst $\alpha_{\mathrm{CO}}=0.8 \mathrm{M}_{\odot} \mathrm{pc}^{-2}\left(\mathrm{~K} \mathrm{~km} \mathrm{~s}^{-1}\right)$ for our sample only yields:

$$
\log _{10}\left(\Sigma_{\mathrm{SFR}}\right)=(1.61 \pm 0.07) \log _{10}\left(\Sigma_{\mathrm{mol}}\right)-(4.15 \pm 0.22)
$$

In both cases, the data appear to support the "two sequences" idea, at least to some degree, with internal relationships in the two sub-samples that are more nearly linear, and a steep slope when contrasting both populations. This is particularly the case when we use a starburst conversion factor for our sample.

Adopting $\alpha_{\mathrm{CO}} \propto \Sigma_{\text {mol }}^{-0.5}$ (see equation 3.4) we find instead

$$
\log _{10}\left(\Sigma_{\mathrm{SFR}}\right)=(1.88 \pm 0.06) \log _{10}\left(\Sigma_{\mathrm{mol}}\right)-(4.64 \pm 0.19)
$$

In this case we find an even steeper slope when fitting the combined data, from our U/LIRGs and the normal spirals from Leroy et al. (2013), than when we use a starburst conversion factor for our sample only, and even more so when we fit either sample alone. To some degree, this reinforces the "two sequences" view and it strongly reinforces the idea that the disk-starburst contrast is essential to probe the non-linear nature of star formation scaling relations. We also show, following a number of others (e.g., see Bouché et al. 2007; Ostriker \& Shetty 2011) that the adopted conversion factor, in addition to the starburst sizes, plays a large role in the results.

Efficiency per Free Fall Time: A popular class of models posits an approximately fixed fraction of gas converts to stars per gravitational free fall time, $\tau_{\mathrm{ff}}^{\mathrm{mol}}=\sqrt{3 \pi /\left(32 \mathrm{G} \rho_{\mathrm{mol}}\right)}$ (e.g., Krumholz \& McKee 2005b; Krumholz et al. 2012). If we adopt a simple, spherical, with radius $R_{50, d}$, view of the geometry of our systems, we can estimate $\tau_{\mathrm{ff}}^{\mathrm{mol}}$. For a three dimensional Gaussian, this implies an aperture correction of $\sim 1 / 3.4$ for the total gas mass (or SFR) within that volume.

Comparing $\tau_{\mathrm{ff}}^{\mathrm{mol}}$ to the depletion time of the molecular gas mas, $\tau_{\mathrm{dep}}^{\mathrm{mol}}$, we estimate the efficiency of the conversion of the gas mass into stars per free fall time, or $\epsilon_{\mathrm{ff}}=\tau_{\mathrm{ff}}^{\mathrm{mol}} / \tau_{\mathrm{dep}}^{\mathrm{mol}}$. We find median values for $\tau_{\mathrm{ff}}^{\mathrm{mol}}$ of $1.2,1.6$, and $0.5 \mathrm{Myr}$ for "starburst", surface-density dependent, and Galactic conversion factors. These numbers 
imply median $\epsilon_{\mathrm{ff}}$ of $9 \%, 19 \%$, and $0.7 \%$. These numbers appear high compared to the universal $\sim 1 \% \epsilon_{\mathrm{ff}}$ assumed in the Krumholz et al. (2012) model, and in more agreement with a non-universal star formation efficiency (Semenov et al. 2015), but we emphasize the uncertainty in our adopted geometry.

\subsubsection{Are Local Major Mergers Maximal Starbursts?}

The extreme environments present in the local U/LIRGs of our sample are undoubtedly subject to feedback processes that can halt the ongoing star formation. Scoville (2003); Murray et al. (2005); Thompson et al. (2005) proposed radiation pressure on dust as the main feedback mechanism for compact, optically thick starbursts, such as the local starbursts we study in this paper ${ }^{9}$. The high $\Sigma_{\mathrm{IR}}$, or $\Sigma_{\mathrm{SFR}}$, values derived for these systems indicate they may be close to or above the maximal starburst limit. This limit represents the maximum brightness a source can have that can be produced only by star formation. If a source is above this limit, then an external source of energy, such an AGN, has to be invoked in order to explain such high values.

In Figure 3.10, we explore this by comparing $\Sigma_{\mathrm{IR}}$ of our systems to the theoretical values presented by Thompson et al. (2005) in their Figure 4. These limits depend on the size, stellar velocity dispersion $(\sigma)$, gas fraction $\left(f_{g}\right)$, dust-to-gas ratio, and Rosseland mean opacity $(\kappa)$ of the system, which imply several assumptions when determining the true "Eddington limit". Assuming in the center of these local $\mathrm{U} /$ LIRGs $\mathrm{f}_{\mathrm{g}}=1$ and $\sigma \approx 200 \mathrm{kms}^{-1}$ (e.g., Genzel et al. 2001), indicated by the solid green line, we observe in Figure 3.10 that at least 4 systems are at, or above, the Eddington limit. These are Mrk 231 (\#10), Arp 220 (\#17), IRAS 15250+3608 (\#16), and IRAS F01364-1042 (\#2). Mrk 231, an optical Seyfert 1, is the one deviating the most from the assumed Eddington limit. The other 4 sources which are close to the limit have mid-IR evidence of energetic AGN - i.e., low PAH equivalent widths (= 0.01-0.17) and/or high 30-to-15 $\mu \mathrm{m}$ flux density ratios (=10-33) indicative of very warm, Seyfert-like mid-IR dust emission (Stierwalt et al. 2013). In terms of Arp 220, there is some tension with our prior results presented in Barcos-Muñoz et al. (2015),

\footnotetext{
${ }^{9}$ Another feedback mechanism that can be important in compact starbursts is cosmic ray pressure (e.g., Socrates et al. 2008).
} 

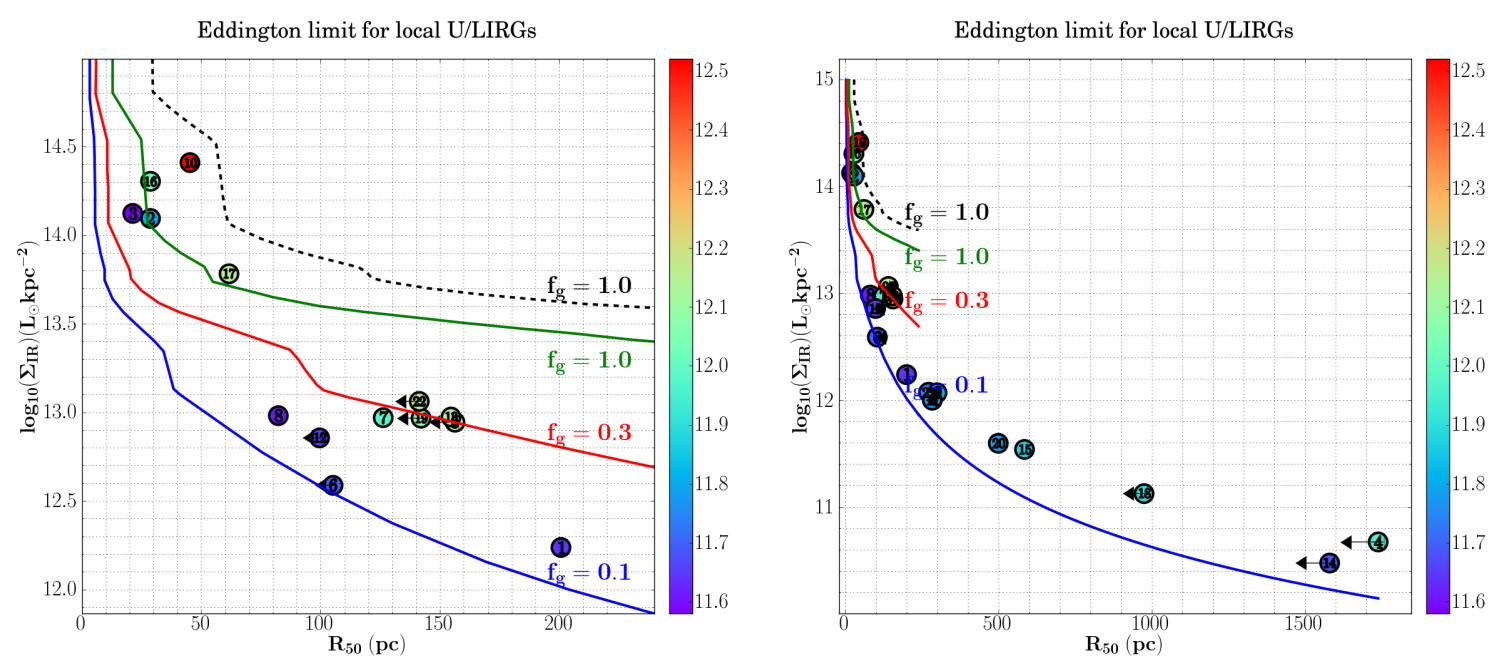

Fig. 3.10.- - Infrared surface brightness versus radius for local U/LIRGs. Lower limits are indicated with an arrow. This is an updated version of Figure 3 from Barcos-Muñoz et al. (2015) adapted from Figure 4 in Thompson et al. (2005). The solid lines represent the Eddington limits presented by Thompson et al. (2005) for different gas fractions $\left(\mathrm{f}_{g}\right)$ and a stellar velocity dispersion sigma $=200 \mathrm{~km} \mathrm{~s}^{-1}$. The dashed line represents the Eddington limit for $\mathrm{f}_{g}=1$ and sigma $=300 \mathrm{~km} \mathrm{~s}^{-1}$. Assuming the green solid line is the proper "Eddington limit", one source shows clearly super Eddington values (\#10, Mrk 231). Other three sources are very close to the "Eddington limit", including IRAS F01364-1042, Arp 220, and IRAS 15250+3608, although the value for Arp 220 is most likely inflated due to lack of geometry corrections (see Section 3.5.5 for further discussion). The majority of the systems are not maximal starbursts. The left panel shows the more extended systems in a zoomed out version of the plot in the right panel.

where we found that each nucleus in Arp 220 is far from the Eddington limit. One possible explanation is related to the geometry of the source and the fact that we are considering both nuclei as a single source. If we take these considerations into account we would need to decrease the measured $\Sigma_{\mathrm{IR}}$ here by at least $1 / 2$, which would place Arp 220 immediately below the Eddington limit (see Appendix A in Barcos-Muñoz et al. 2015, for further discussion on geometry corrections). We consider the other two sources to be maximal starbursts. The rest of our sample is well below the Eddington limit assumed here, although further knowledge on the proper gas fraction, opacity, and stellar velocity of each system is needed to make a statement on the mode these U/LIRGs are producing stars, and whether they are maximal starbursts.

\subsection{Conclusions}

We present a high resolution imaging survey of $33 \mathrm{GHz}$ continuum emission from local U/LIRGs. Using all four VLA configurations and a bandwidth of $\Delta \nu=2 \mathrm{GHz}$, 
we achieve very high resolutions of $0{ }^{\prime} .07-00^{\prime} 67$, or $30-720$ pc at the distance of our sample while still retaining sensitivity to emission on large scales. This is the first such survey at high frequency (for the VLA) and as a result, the resolution improves on previous work by Condon et al. (1991b) and Condon et al. (1990). Because of the steep spectral index of galaxies in this range, the improved sensitivity gained from the VLA upgrade was a key element in the survey. Using these data, we find:

1. Most of the $33 \mathrm{GHz}$ emission observed at low resolution arises from sources that appear compact in our highest resolution maps. For the majority of our targets, more than $50 \%$ of the integrated flux density at $33 \mathrm{GHz}$ arises from sources with Gaussian-like morphologies at high resolution and extent typically a few times the size of the beam.

2. The $33 \mathrm{GHz}$ emission reflects a mixture of synchrotron and free-free emission. For different approaches we achieve slightly different results, but within the uncertainties approximately equal fractions of thermal and nonthermal emission could contribute at $33 \mathrm{GHz}$. To improve on this uncertain number improved coverage of the radio SED, especially achieving reliable flux densities at many frequencies in the range 15-50 GHz, will be extremely helpful. Unless the emission is highly clumped within our high resolution beam, brightness temperature arguments suggest that all of our targets are optically thin at $33 \mathrm{GHz}$.

3. By making use of the $33 \mathrm{GHz}$ size to indicate the active, star-forming region, we provide estimates for the surface densities of gas, star formation, and infrared emission. These quantities are all more extreme than those found in typical starforming galaxies but also vary strongly across the sample, spanning a range of $\approx 4$ dex. The highest values in our sample are among the highest measured for any galaxies.

4. We also make use of the measured $33 \mathrm{GHz}$ sizes of the sample to estimate their star formation rate surface densities, $\Sigma_{\mathrm{SFR}_{33 \mathrm{GHz}}}$. We find that $L_{[\mathrm{CII}]} / L_{\mathrm{FIR}}$ decreases with increasing $\Sigma_{\mathrm{SFR}_{33 \mathrm{GHz}}}$, increasing opacity (as measured via the flattening of the radio spectral index between 1.5 and $6 \mathrm{GHz}$ ), and increasing 
compactness. These measurement agree with prior studies which make use of infrared sizes to estimate $\Sigma_{\mathrm{IR}}$ (or $\Sigma_{\mathrm{SFR}}$ ), and they confirm that the [C II] 'deficit' is more pronounced in the most compact and obscured U/LIRGs.

5. We consider the implications for star formation scaling relations from $\Sigma_{\mathrm{SFR}}$ and $\Sigma_{\text {gas }}$ derived combining our size estimates with unresolved CO and IR observations. For any single, fixed conversion factor and considering only our sample, we find a slope near unity $(\approx 1.04)$ relating the two. However, our targets contrast with results for normal spiral galaxies from (Leroy et al. 2013), and a nonlinear slope is needed to relate the two different populations (consistent with Kennicutt 1998; Liu et al. 2015).

6. The exact value of the power law index needed to fit both normal disks and U/LIRGs depends sensitively on the sizes of the U/LIRGs (which we know) and the prescription for the CO-to- $\mathrm{H}_{2}$ conversion factor (which we do not). We show results for three common approaches to the conversion factor, and the power law index relating normal disk galaxies to our U/LIRGs varies from $\sim 1.3$ to $\sim 1.8$. For any fixed conversion factor, our data do offer some support for the idea of two sequences of star formation, though the contrast is weaker when the conversion factor is the same for both samples.

7. The high column densities that we infer imply high opacities outside the $\sim \mathrm{cm}$ and mm-wave regime. By adopting a "starburst" conversion factor, the average extinction at optical wavelengths is $A_{V} \sim 17-3 \times 10^{4}$ mag for our targets. 12 of our sources appear Compton thick, with average $\mathrm{N}_{\mathrm{H}}>1.5 \times 10^{25} \mathrm{~cm}^{-2}$. At IR wavelengths, the opacity is less, $\tau_{100} \sim 0.02--30$, however they are still affected by dust with those same 12 sources being optically thick at $100 \mu \mathrm{m}$. Applied to the measured $1.5 \mathrm{GHz}$ flux densities, our sizes also indicate that opacity must play a significant role at lower radio frequencies.

8. Our targets show high infrared surface brightnesses, with 12 sources having $\Sigma_{\text {IR }}>10^{13} \mathrm{~L}_{\odot} \mathrm{kpc}^{-2}$. These values are close to the "maximal" starburst limit set by radiation pressure on dust. However, by comparing to models from 
Thompson et al. (2005), we find most of our targets are not "maximal" starburst. There are three exceptions: Mrk 231 showing a super-Eddington value, and IRAS 15250+3608 and IRAS F01364-1042 that are at the Eddington limit.

\section{Acknowledgments}

A.S.E., G.C.P. and L.B-M. were supported by NSF grant AST 1109475. L.B-M. was also supported by Fulbright, Becas Chile - CONICYT and through the Grote Reber predoctoral fellwoship from NRAO. This research made use of the NASA/IPAC Extragalactic Database (NED), which is operated by the Jet Propulsion Laboratory, California Institute of Technology, under contract with the National Aeronautics and Space Administration, and NASA's Astrophysics Data System Bibliographic Services. The National Radio Astronomy Observatory is a facility of the National Science Foundation operated under cooperative agreement by Associated Universities, Inc. 
Appendix 


\section{A Notes on the sources}

CGCG 436-030: This system has too well separated components (east and west), however we only detected the western component at $33 \mathrm{GHz}$.

CGCG 448-020: This is an interacting system showing a complex morphology. It is still not clear whether there are two or more systems interacting. It hosts an offnuclear starburst (north-east component in Figure 3.1) which contributes $\sim 80 \%$ of the total infrared luminosity of the galaxy at infrared wavelengths (Stierwalt et al. in prep). In our final map (i.e., the one with the highest resolution, not shown in this work), this off-nuclear starburst is still only partially resolved, even at 0 '.08, while the more extended component (south-west) is resolved out.

III Zw 035: This is the galaxy showing the most compact $33 \mathrm{GHz}$ continuum emission, even though it is not a U/LIRG.

IRAS 15250+3608: This systems is one of the sources emitting at, or close to, the Eddington limit. The optical and mid-IR diagnostics classify this galaxy as a composite source. The fact that it is close to the Eddington limit, agrees with the potential coexistence of an AGN and a strong starburst.

IRAS 17132+5313: This system has two components. The galaxy towards the north east is extended and resolved out in the highest resolution image $\left(00^{\prime} 08 \times\right.$ $0.07)$. We had to taper the map in order to recover its emission. The galaxy towards the south west is compact and contributes $\sim 40 \%$ of the integrated flux density of the system.

NGC 3690: This is a system with clear signs of interaction showing multiple components. Two of them are associated with the nuclei of the progenitors, NGC 3690E (east) and NGC 3690W (west), while the others are a combination of off nuclear starbursts and a PG QSO. The strongest nucleus (NGC 3690E) has been observed with VLBI. At least 30 point sources have been found plus a potential AGN (e.g., Neff et al. 2004). Due to the proximity of this system and its spatial extent, the 33 $\mathrm{GHz}$ emission is resolved at the D configuration resolution $(\sim 2$ "), clearly showing 5 components (see red crosses in map from Figure 3.1). In order to measure its total flux density, we tapered the D configuration map until the system showed 2 unre- 
solved components (east and west systems). We then proceed as explained in Section 3.3.1, by fitting a Gaussian to each one.

UGC 04881: This system has two components and its total flux density was recovered by adding the Gaussian fit results of each component separately. The error of this measurement was obtained by adding in quadrature the errors associated to each component (see Section 3.3.1). The D configuration map of this system had low signal-to-noise and the quality was not good enough to recover the total flux density. For this reason, we used the final image (with the different array configurations combined, see Section 3.2) tapered such that we recovered a point-like structure for each component. Even though both components contribute about the same to the total flux density observed at $32.5 \mathrm{GHz}$, the brightest component (north-east) is more compact. The south-west component is resolved out at the highest resolution we can achieve. We measured the size of this component from the image we used to obtain the total flux density $\left(\mathrm{A}_{\text {beam }}=20.3 \mathrm{arcsec}{ }^{2}\right)$ and found an upper limit of $\mathrm{A}_{50}=19.8 \operatorname{arcsec}^{2}$, i.e., it is unresolved in this coarser map. The brightest component is shown in Figure 3.1.

UGC 08058: This is the most powerful source in our sample. It is known to host an AGN (e.g., Lonsdale et al. 2003; Iwasawa et al. 2009) and potentially represents the stage before becoming an elliptical galaxy according to the evolutionary model proposed by Sanders et al. (1988b).

Arp 220: This is the closest U/LIRG in the local universe. This galaxy shows extreme dust opacities and very compact nuclear disks. We present a detailed analysis of the 33 and $6 \mathrm{GHz}$ emission from this galaxy in Barcos-Muñoz et al. (2015), where we find that the disks are better described by exponential disks, rather than Gaussian. The $33 \mathrm{GHz}$ map reported in Barcos-Muñoz et al. (2015) is slightly different to the one presented here since the imaging procedures differ, however the flux density measured here and the morphology are in agreement with those shown in Barcos-Muñoz et al. (2015).

VII Zw 031: At the highest resolution image $\left(0^{\prime \prime} .8 \times 0^{\prime \prime} 6\right.$, done with natural weighting) the emission was completely resolved out. We had to taper the image heavily in order to recover the emission. This is one of the most extended systems in our sample 
along with VV 340a.

VV 250: This system has two well separated components, south-east (VV 250a) and north-west (VV 250b). In Figure 3.1, we only show VV 250a since it concentrates $\sim 86 \%$ of the total flux density of the system (obtained by adding the flux density of both components). The north-west component is faint with an $11 \sigma$ peak detection. To recover $\mathrm{A}_{50}$ for this faint component, we used the tapered $\mathrm{D}$ array map $(\sim 10$ " resolution) since we could not recover half of the integrated flux density of this component in higher resolution maps. Even in this low resolution map, we recover $\mathrm{A}_{50}$ for $\mathrm{C}_{50}=3.2 \sigma$, which is lower than our conservative limit of $5 \sigma$, however we inspected this contour and made sure the emission within it looked real. For the north-west component, $\mathrm{A}_{50}=64.8 \operatorname{arcsec}^{2}$ in a map with $\mathrm{A}_{\text {beam }}=113.4 \operatorname{arcsec}^{2}$, i.e., it is unresolved, and then $\mathrm{A}_{50}$ is only an upper limit.

VV 340a: In the final combined image, where we achieved a spatial resolution of $0.5 \times 0.4$ (using natural weighting), the emission from this system was completely resolved out. To recover the extended emission, we had to taper the image heavily. VV 340 has two components, an edge on galaxy to the north (VV 340a), shown in Figure 3.1, and a face on galaxy to the south (VV 340b). Inconveniently, the pointing of the VLA observation was centered on VV 340b, from which we tentatively detected an off nuclear feature at a $\sim 3 \sigma$ level in our lowest resolution image. The bright edge on galaxy, VV 340a, is clearly detected, although it was hard to perform the Gaussian fit since the source fell close to the edge of the primary beam.

VV 705: This system shows two nuclei in Figure 3.1, north-west and south-east. In the D configuration map they are indistinguishable. 
Chapter 4

ALMA View of the Arp 220 Disks

From New Extended Configuration Observations of Dense Gas Tracers 


\begin{abstract}
We present new high-resolution continuum and line observations of the prototypical (and closest) ULIRG, Arp 220. Arp 220 is one of the most extreme local star-forming systems and is often used as a template for starbursts at high redshift. Using the most extended configuration of ALMA available at Cycle 3, we achieve resolution of $0^{\prime \prime} 08=30 \mathrm{pc}$ at Band 3 , targeting the mm-wave $(\sim 90 \mathrm{GHz})$ continuum and the high critical density tracers $\mathrm{HCN}(1-0), \mathrm{HCO}^{+}(1-0)$, their isotopologues, and the shock tracer $\mathrm{SiO}(2-1)$. This resolution is sufficient to resolve both disks and ideal to compare to our $33 \mathrm{GHz}$ VLA continuum images at the same resolution (BarcosMuñoz et al. 2015). The continuum emission at $92 \mathrm{GHz}$ shows similar distribution when compared to the $33 \mathrm{GHz}$ map, indicating the $92 \mathrm{GHz}$ emission in Arp 220 is optically thin and traces star formation. We also combine the two data sets to construct resolved spectral flux density distributions maps of the two nuclear disks hosting the energetically dominant regions of the galaxy. From their spectral flux density distribution, the two nuclei show distinct behaviors, with the west showing a rising spectral index through the $100 \mathrm{GHz}$ regime, indicative of opaque free-free or dust emission. The eastern nucleus shows a flatter spectrum implying thermal emission is still an important component at this high frequencies. In fact, taking into account that we only recover $60 \%$ of the total flux density at $92 \mathrm{GHz}, 40 \%$ of the emission in the western nucleus at $92 \mathrm{GHz}$ is due to thermal dust, while $56 \%$ of the emission in the eastern nucleus is due to free-free emission. Using the line emission, which traces the high density gas pervasive in the system, we present gas and kinematic profiles for both disks. We observe clear evidence for nuclear P-Cygni profiles with characteristic velocities, corrected for inclination, of 170 and $80 \mathrm{~km} \mathrm{~s}^{-1}$ for the east and west nucleus. These imply mass outflow rates of 3400 and $1600 \mathrm{M}_{\odot}$ $\mathrm{yr}^{-1}$ and mass loading factors of 35 and 18, which suggest an AGN is helping boost these outflow rates, however these numbers are strongly dependent on the HCN-togas mass conversion factor, and should be only used as upper limits. We also observe the dense gas is rotating in both nuclei, with the west nucleus also showing rotation signatures from a potential outer gas envelope. Absorption features are strongest in
\end{abstract}


the western nucleus and affect more the $\mathrm{HCO}^{+}$emission.

\subsection{Introduction}

At a luminosity distance of $77 \mathrm{Mpc}$ and $\mathrm{L}_{\mathrm{IR}}[8-1000 \mu \mathrm{m}] \sim 10^{12.2} \mathrm{~L}_{\odot}$, Arp 220 is the closest ultraluminous infrared galaxy (ULIRG: $\mathrm{L}_{\mathrm{IR}}[8-1000 \mu \mathrm{m}] \geq 10^{12} \mathrm{~L}_{\odot}$ ) and one of the most extreme local star-forming system (e.g., as the prototype for "maximal" starbursts). Due to its high infrared luminosity, extreme physical conditions, and proximity, it has been well studied at many wavelengths. It is considered a prototype to understand more distant ULIRGs and is frequently used as a template for starbursts at high redshift.

Despite decades of study, Arp 220's extreme opacity and compactness mean that it is still mysterious in many ways. The system is a late-stage merger that hosts two extremely compact nuclei each with less than 1" of angular diameter and only separated by $\sim 1^{\prime \prime} \approx 369$ pc (e.g., Sakamoto et al. 1999; Barcos-Muñoz et al. 2015). Each nucleus harbors gas and dust comparable to the entire content of some galaxies $\left(\sim 10^{9} \mathrm{M}_{\odot}\right)$, rendering them optically thick even at mid-IR wavelengths (e.g., Armus et al. 2007). Opacity is even present at radio wavelengths, with free-free absorption playing an important role at $\sim \mathrm{GHz}$ frequencies.

These characteristics conspire to set a very limited range of frequencies where Arp 220 is optically thin (from about 5 to $350 \mathrm{GHz}$, see Barcos-Muñoz et al. 2015). This makes observations of the innermost regions of this key system very challenging and explains why major uncertainties still surround even the basic structure of the nuclei. For example, we have little idea of how the dominant radio emission mechanism varies across the inner disks, what the vertical velocity dispersion of either disk is (a key measure of disk structure), and even what the inner portion of the rotation curve looks like in each nucleus. These factors bear directly on whether Arp 220 is actually a "maximal" starburst limited by feedback processes, and/or if supermassive black holes contribute significantly to the mass and luminosity of the system.

The nuclei of Arp 220 have been observed multiple times in the $\mathrm{cm}-\mathrm{mm}$ regime, but mostly with resolution comparable to the size of the nuclei (e.g., Norris 1988; 
Condon et al. 1991b; Downes \& Solomon 1998; Sakamoto et al. 2008; Wilson et al. 2014). Similarly, efforts to observe the kinematics of molecular and ionized gas have either lacked angular resolution (e.g., Imanishi et al. 2007; Sakamoto et al. 2008; Greve et al. 2009; Tunnard et al. 2015; Scoville et al. 2015) and/or targeted regimes with optically thick continuum (e.g., Rangwala et al. 2015). Before this year, the highest resolution published kinematic studies at an optically thin regime (to our knowledge) remain Sakamoto et al. $(2008,2009)$ and the recently published results by Scoville et al. (2015) with $\sim 0.5 \%$. In these cases, continuum emission of the nuclei is distinguishable, but the kinematic information is not good enough to measure inner rotation curves for the individual disks.

As part of its first "extended configuration" campaign, ALMA observed Arp 220 at $\nu \sim 100 \mathrm{GHz}$ with $\sim 0^{\prime \prime} 1$ as part of two projects. Scoville et al. (2016) recently published observations targeting the $\mathrm{CO}(1-0)$ emission, tracing the whole gas distribution. Here, we present the first highly resolve maps of $\mathrm{HCN}$ and $\mathrm{HCO}^{+}$emission in Arp 220 (PI: Barcos-Muñoz, Project Code 2015.1.00702.S). These were designed to trace the motions of dense gas in the immediate vicinity of the two nuclei, yielding, where possible, dynamical constraints on the distribution of mass, as well as evidence of feedback. At the same time, we aimed to image the continuum emission to compare with our $33 \mathrm{GHz}$ data and to constrain the emission mechanisms within the nuclei.

This paper is structured as follows: we present the observations and explain data reduction process in Section 2.2. In Section 4.3, we analyze the continuum emission. In Section 4.4, we measure the line emission, comparing the spectra of the two nuclei, showing signatures of outflowing material, and constraining the rotation and dispersion of the two disks. We conclude in Section 4.5 and note future directions in Section 4.6 .

Throughout this paper, we adopt $\mathrm{H}_{0}=73 \mathrm{~km} \mathrm{~s}^{-1} \mathrm{Mpc}^{-1}, \Omega_{\text {vacuum }}=0.73$ and $\Omega_{\text {matter }}=0.27$, with velocities corrected to the cosmic microwave background frame. The scale at Hubble flow distance is 369 pc/". 


\subsection{ALMA Observations of Arp 220}

We observed Arp 220 in three different sessions between 2015 October 15 and 2015 October 29. The observations were targeted on the high critical density transitions $\mathrm{HCN}(1-0)$ and $\mathrm{HCO}^{+}(1-0)$, their optically thin isotopologues $\mathrm{H}^{13} \mathrm{CN}$ and $\mathrm{H}^{13} \mathrm{CO}^{+}$, and the shock tracer $\mathrm{SiO}(2-1)$.

We used J1550+0527 as the flux density calibrator, and J1532+2344 and J1537+2300 as phase calibrators. The total integration time on source was 2 hours, and 50 antennas were used. All of these observations occurred when ALMA was in the most extended configuration available in Cycle 3, C36-8. This configuration has a maximum baseline of $\approx 10 \mathrm{~km}$. The largest recoverable angular scale is $1.1^{\prime \prime}$.

We configured the correlator to process four spectral windows (SPWs), each 1.875 $\mathrm{MHz}$ wide. Two of these were in the lower sideband (LSB) and the other two were in the upper sideband (USB). The central rest frequency, $\nu_{\text {rest }}$, of the four spectral windows were 86.75, 88.75, 99.022 and $101 \mathrm{GHz}$. This placed our main lines of interest in the lower sideband spectral windows, while the two higher frequency windows targeted lines of opportunity and the mm-wave continuum. After on-line smoothing, the spectral resolution near our lines of interest was $13.6 \mathrm{~km} \mathrm{~s}^{-1}$.

The data reduction, imaging and analysis of the data were carried out using the Common Astronomy Software Application package (CASA; McMullin et al. 2007). We calibrated the raw data by running the observatory-provided reduction script. We then separate the calibrated observations of Arp 220 from the calibration data. We examined the full, calibrated data set using to identify the channels that contained only continuum emission without contamination by spectral lines.

Using this information, we produced continuum images of Arp 220. To do this, we first flagged the channels with line emission, so that we work with only continuum emission. Then, to increase the processing speed, binned the data in frequency by a factor of 40 channels. We imaged the binned data set using the task clean in its multifrequency synthesis (mfs) mode (Sault \& Wieringa 1994) with nterms=2. We used the Cotton-Schwabb algorithm (imagermode csclean) with a Briggs weighting scheme, setting the robust parameter to 0.5 , and using a pixel size of $0{ }^{\prime} 01$. 
Our first continuum image had rms noise of $17 \mu \mathrm{Jybeam}^{-1}$ and a peak signal-tonoise ratio (SNR) of 482. This high SNR was enough that we could perform selfcalibration in order to attempt to solve for short-timescale variations in the phase and amplitude response of the telescopes. We were able to perform three phase self-calibrations and two amplitude self-calibrations. After self-calibration, the final continuum image had an rms noise of $14.3 \mu \mathrm{Jybeam}^{-1}$ and a peak SNR of 638 , so that self-calibration improved the peak SNR by $\approx 30 \%$. The fiducial frequency of the final multifrequency synthesis image was $\nu=92.2 \mathrm{GHz}$ with a restoring beam size of $0{ }^{\prime} .09 \times 0$ 0 ${ }^{\prime} 07$. The total integrated intensity of Arp 220 at $92.2 \mathrm{GHz}$ is $32 \mathrm{mJy}$. In Figure 4.1 we show the $92 \mathrm{GHz}$ continuum map and compare its morphology to that of the $33 \mathrm{GHz}$ continuum map from Barcos-Muñoz et al. (2015).
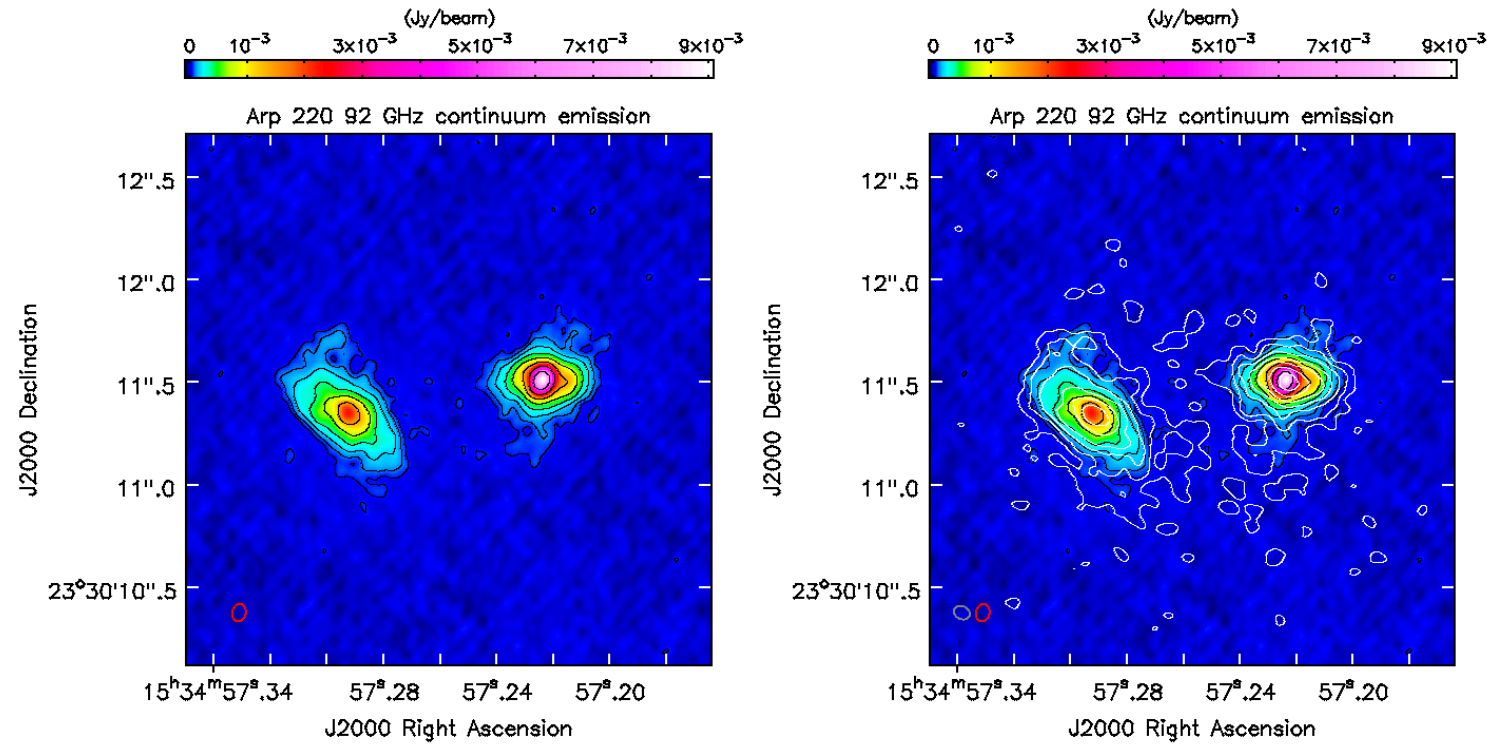

Fig. 4.1.- : (Left) A $92.2 \mathrm{GHz}$ continuum image of Arp 220 with its contours overlaid. The contours are in steps of $2^{\mathrm{n}} \times 3 \sigma$ with $\mathrm{n}=0,1,2, \ldots$ and $\sigma=14.4 \mu \mathrm{Jy}_{\text {beam }}{ }^{-1}$. The FWHM of the restoring beam is $0^{\prime \prime} 09 \times 00^{\prime \prime} 07$, p.a. $\approx-13^{\circ}$ and it is shown in the bottom left corner in red. (Right) Same as left panel, but with white contours from the $33 \mathrm{GHz}$ continuum emission overlaid from Barcos-Muñoz et al. (2015). The contours at $33 \mathrm{GHz}$ are in steps of $2^{\mathrm{n}} \times 3 \sigma$ with $\mathrm{n}=0,1,2, \ldots$ and $\sigma=23 \mu \mathrm{Jy}$ beam $^{-1}$. The FWHM of the restoring beam is $00^{\prime \prime} 08 \times 00^{\prime \prime} 06$, p.a. $\approx 65^{\circ}$ and it is shown to the left of the $92 \mathrm{GHz}$ beam, in grey. We re-grid the $33 \mathrm{GHz}$ using a cubic interpolation in order to match the pixel sizes.

The multifrequency synthesis produces spectral index maps, but to analyze the spectral flux density distribution in more depth, we also produced a separate continuum image using the line-free portion of each SPW (fiducial frequencies $\nu=85.2$, 
87.2, 97.3, and $99.2 \mathrm{GHz})$, and one for the whole lower and upper sideband $(\nu=86$ and $98 \mathrm{GHz}$ ). The total integrated intensity of Arp 220 at $97.3 \mathrm{GHz}$ is $37.9 \mathrm{mJy}$. This indicates we recover only $60 \%$ of the total continuum measured at $97.2 \mathrm{GHz}$ by Anantharamaiah et al. (2000) based on line measurements of $\mathrm{H} 40 \alpha$. Most likely that $40 \%$ missing is extended emission with sizes larger than $1^{\prime \prime}$. (the largest recoverable scale of our observations) that we are not able to detect due to spatial filtering. In Table 4.1 we report the beam size of each image, the integrated flux densities of each nucleus and the entire system and the peak intensity of each nucleus. We also include literature data of such measurements.

To image the line emission, we returned to the original data before flagging and binning. We applied the self-calibration that we derived for the continuum to these line data. On this calibrated measurement set, we carried out a $u-v$ continuum subtraction using the task uvcontsub, fitting the continuum with a polynomial of order 1 . We excluded the previously identified line channels from the fit, so that the subtraction fit and subtracted only the continuum.

We imaged the continuum-subtracted visibility data for each whole SPW in to a single large datacube. To do this, we used clean with mode='velocity' and a Briggs weighting scheme with the robust parameter set to 1.0. We binned the final cube to a spectral resolution of $54.4 \mathrm{~km} \mathrm{~s}^{-1}$, coarser than the native $13.6 \mathrm{~km} \mathrm{~s}^{-1}$. Thanks to the very wide lines in the Arp 220 nuclei, this resolution is still sufficient to resolve the lines, and the averaging involved increases the SNR by a factor of $\approx 2$.

Figures 4.2 and 4.3 show the continuum-subtracted spectra. The first shows spectra integrated over an aperture enclosing the $3 \sigma$ continuum intensity contour for each nucleus (see Figure 4.1). The second shows spectra from an individual beam at the peak of the continuum emission of each nucleus. In each figure, we label notable lines, which we list in Table 4.2. 
Table 4.1. Flux densities at different frequencies for Arp 220

\begin{tabular}{|c|c|c|c|c|c|c|c|}
\hline \multirow{2}{*}{$\begin{array}{c}\text { Frequency } \\
(\mathrm{GHz})\end{array}$} & \multirow{2}{*}{$\begin{array}{l}\text { Beam } \\
\left({ }^{\prime \prime} \times{ }^{\prime \prime}\right)\end{array}$} & \multirow{2}{*}{$\begin{array}{l}\text { Total } \\
(\mathrm{mJy})\end{array}$} & \multicolumn{2}{|r|}{ East } & \multicolumn{2}{|r|}{ West } & \multirow[t]{2}{*}{ Reference } \\
\hline & & & $\begin{array}{c}\text { Integrated } \\
(\mathrm{mJy})\end{array}$ & $\left(\mathrm{mJy} \mathrm{beam}^{-1}\right)$ & $\begin{array}{c}\text { Integrated } \\
(\mathrm{mJy})\end{array}$ & $\begin{array}{c}\text { Peak } \\
\left(\mathrm{mJy} \mathrm{beam}^{-1}\right)\end{array}$ & \\
\hline 4.7 & $0.60 \times 0.43$ & 222.0 & 92.4 & 61.8 & 114.6 & 89.5 & Barcos-Muñoz et al. (2015) \\
\hline 5.95 & $0.38 \times 0.28$ & 197.6 & 81.4 & 49.0 & 94.3 & 73.3 & Barcos-Muñoz et al. (2015) \\
\hline 7.2 & $0.38 \times 0.28$ & 171.4 & 73.2 & 36.0 & 89.5 & 60.4 & Barcos-Muñoz et al. (2015) \\
\hline 32.5 & $0.081 \times 0.063$ & 61.8 & 30.1 & 4.1 & 33.4 & 6.5 & Barcos-Muñoz et al. (2015) \\
\hline 85.2 & $0.098 \times 0.074$ & 34.67 & 12.78 & 2.58 & 21.23 & 9.01 & This work \\
\hline 86.2 & $0.095 \times 0.070$ & 36.16 & 13.40 & 2.46 & 22.27 & 8.94 & This work \\
\hline 87.15 & $0.095 \times 0.069$ & 36.25 & 13.26 & 2.43 & 22.27 & 9.06 & This work \\
\hline 92.2 & $0.087 \times 0.066$ & 36.92 & 13.10 & 2.28 & 23.50 & 9.11 & This work \\
\hline 97.26 & $0.085 \times 0.064$ & 37.90 & 13.06 & 2.24 & 24.36 & 9.49 & This work \\
\hline 98.23 & $0.095 \times 0.070$ & 37.56 & 12.60 & 2.44 & 24.65 & 10.3 & This work \\
\hline 99.20 & $0.084 \times 0.064$ & 36.49 & 11.49 & 2.23 & 24.03 & 9.81 & This work \\
\hline 112.3 & $0.10 \times 0.08$ & $\ldots$ & 16.1 & 3.1 & 29.0 & 13.1 & Scoville et al. (2016) \\
\hline 230.6 & $0.56 \times 0.51$ & 208 & 66 & $\ldots$ & 142 & $\ldots$ & Sakamoto et al. (1999) \\
\hline 341.8 & $0.55 \times 0.40$ & $\ldots$ & 157 & 111 & 328 & 261 & Scoville et al. (2015) \\
\hline 344.6 & $0.51 \times 0.48$ & 780 & 200 & 180 & 380 & 350 & Sakamoto et al. (2008) \\
\hline 691 & $0.36 \times 0.20$ & 4370 & 1510 & $\ldots$ & 1810 & $\ldots$ & Wilson et al. (2014) \\
\hline
\end{tabular}

Table 4.2. Summary of the lines detected by ALMA

\begin{tabular}{cc}
\hline \hline Line & $\nu_{\text {rest }}(\mathrm{GHz})$ \\
\hline $\mathrm{HC}^{15} \mathrm{~N}(1-0)$ & 86.054 \\
$\mathrm{H}^{13} \mathrm{CN}(1-0)$ & 86.34016 \\
$\mathrm{H}^{13} \mathrm{CO}^{+}(1-0)$ & 86.75429 \\
$\mathrm{SiO}(2-1 ; \mathrm{v}=0)$ & 86.84696 \\
$\mathrm{C}_{2} \mathrm{H}(\mathrm{N}=1-0 ; \mathrm{J}=3 / 2-1 / 2)$ & 87.31690 \\
$\mathrm{C}_{2} \mathrm{H}(1-0 ; 1 / 2-1 / 2)$ & 87.40199 \\
$\mathrm{HNCO}\left(4_{0,4}-3_{0,3}\right)$ & 87.92524 \\
$\mathrm{HCN}(1-0)$ & 88.63160 \\
$\mathrm{HCO}(1-0)$ & 89.18852 \\
$\mathrm{HC}_{3} \mathrm{~N}(11-10)$ & 100.0764 \\
$\mathrm{HC}_{3} \mathrm{~N}\left(11-10 ; \mathrm{v}_{7}=1 ; \mathrm{l}=1 \mathrm{e}\right)$ & $100.3224^{\mathrm{a}}$ \\
$\mathrm{HC}_{3} \mathrm{~N}\left(11-10 ; \mathrm{v}_{7}=1 ; \mathrm{l}=1 \mathrm{f}\right)$ & $100.4662^{\mathrm{a}}$ \\
$\mathrm{NH}_{2} \mathrm{CN}^{\mathrm{b}}$ & 100.6295 \\
\hline
\end{tabular}

Note. - Values of the rest frequencies of the lines are from Meier et al. (2015) unless otherwise indicated.

${ }^{a}$ Rest frequencies from Splatalogue.

${ }^{\mathrm{b}}$ Possible ID. 

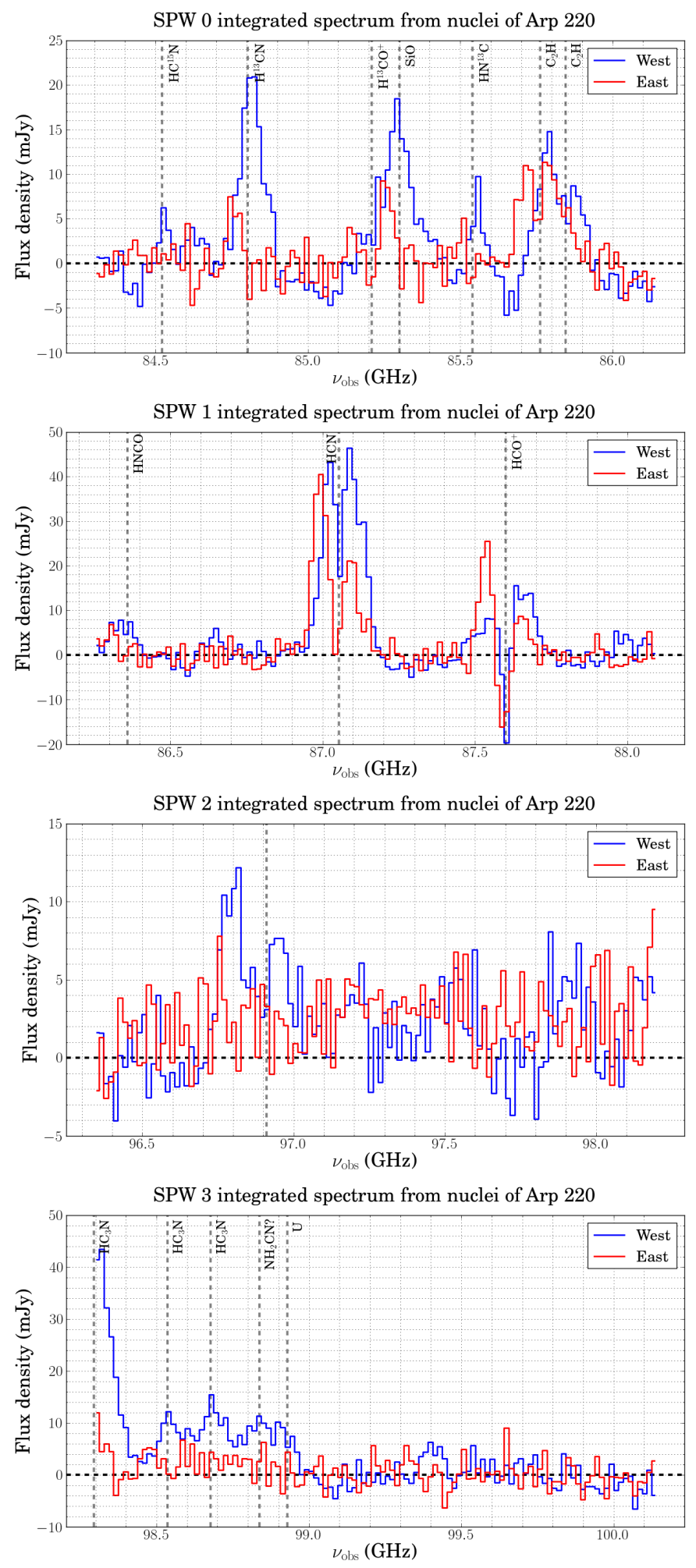

Fig. 4.2.- : Continuum subtracted integrated spectra of the west (blue) and east (red) nucleus per SPW. We mark the location of lines with dashed grey lines. The spectral resolution is $54.4 \mathrm{~km} \mathrm{~s}^{-1}$, which is 4 times the original resolution. No spectral lines were evidently detected in SPW 2, however we mark the location of a line that is tentatively present in the $\mathrm{W}$ nucleus. 

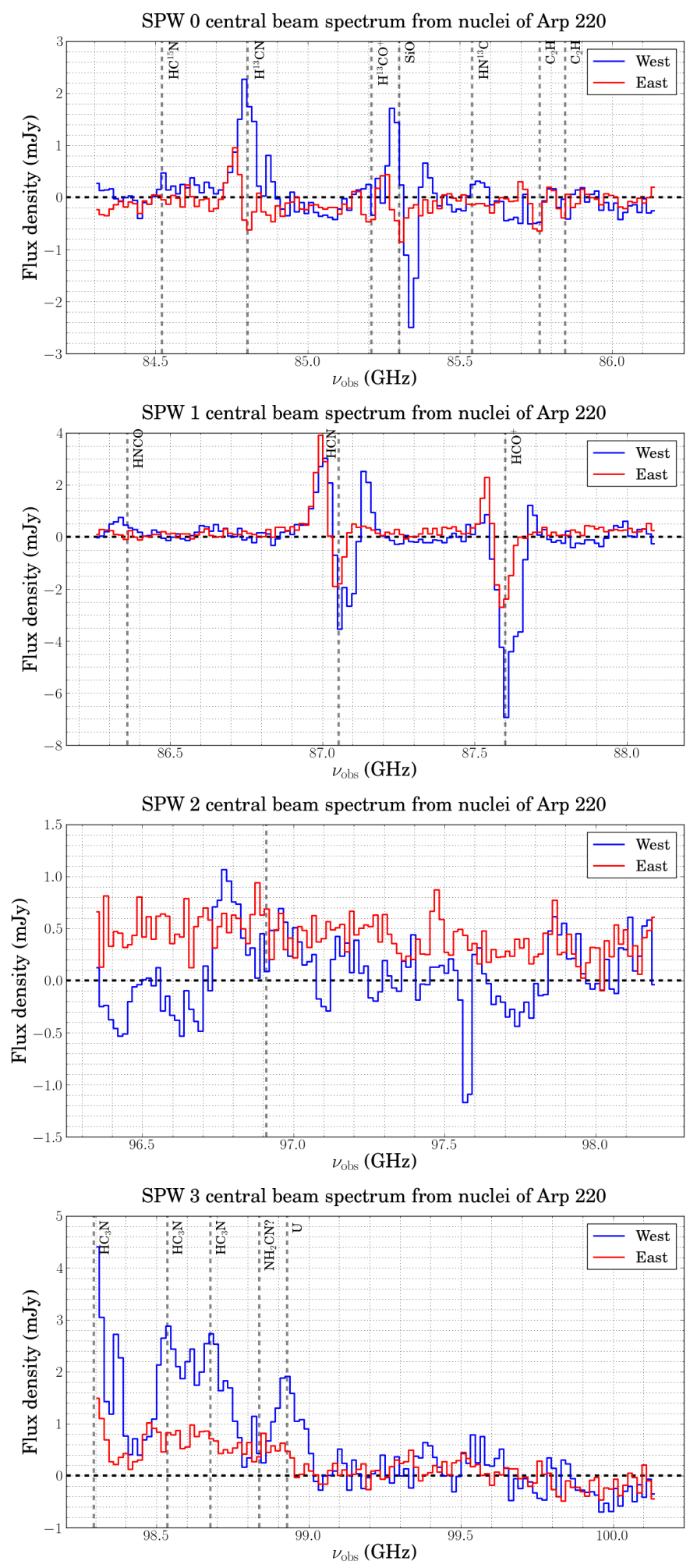

Fig. 4.3.- : Continuum subtracted spectra from a beam-sized region centered in the western (red) and eastern nucleus (blue). No spectral lines were evidently detected in SPW 2, however we mark the location of a line that is tentatively present in the $\mathrm{W}$ nucleus from the integrated spectra shown in Figure 4.2. 
For each line detected in the whole-SPW data cubes, we extracted an individual subcube containing only that line using the CASA task imsubimage. For each of these, we references velocities in the new cube to the rest frequency of the line, using the list reported in Meier et al. (2015) and Splatalogue ${ }^{1}$ as references. We used the CASA routine imreframe, these rest frequencies, a Barycentric reference frame, and the default velocity radio convention for the Doppler shift. After this, each cube was primary beam corrected and smoothed to have a single resolution, which was set by the largest beam present in the original cube.

\subsection{Continuum and Spectral Flux Density Distri- bution}

In Figure 4.1 we show the high resolution, primary beam corrected, continuum image of Arp 220 at $92 \mathrm{GHz}(3.3 \mathrm{~mm})$. The eastern (E) and western (W) nucleus are well resolved and the morphology of the emission follows closely that of the $33 \mathrm{GHz}$ emission (see right panel in Figure 4.1). The $3 \sigma$ contour at $33 \mathrm{GHz}$ is slightly more extended than the $3 \sigma$ contour at $92 \mathrm{GHz}$. A plausible explanation is that the extended emission at $33 \mathrm{GHz}$ could be produced mostly by synchrotron radiation which becomes less important at higher frequencies, where cosmic ray (CR) electrons die faster and thus do not propagate as far as the $33 \mathrm{GHz} \mathrm{CR} \mathrm{e}^{-}$. For higher signalto-noise emission (inner contours), the maps look practically the same however a straightforward explanation for this is not evident. The radio/sub-mm spectrum is a complex mixture of synchrotron from cosmic ray electrons $\left(\mathrm{S}_{\mathrm{nt}} \propto \nu^{-0.8}\right)$, free-free (ionized gas, $\mathrm{S}_{\mathrm{ff}} \propto \nu^{-0.1}$ ), and thermal dust emission ( $\mathrm{S}_{\text {dust }} \propto \nu^{\gamma}$ with $\gamma$ in the range $1-4$ ), and at $\approx 90 \mathrm{GHz}$ all three contribute to the spectrum (see Figure 4.4). From a general perspective, the similarity between the $33 \mathrm{GHz}$ map and the one at $92 \mathrm{GHz}$ indicates the latter is optically thin and traces star formation.

Flux density at $\mathrm{cm}-\mathrm{mm}$ wavelengths is proportional to the frequency to the minus spectral index power, i.e., $\mathrm{S}_{\nu} \propto \nu^{-\alpha}$. Since the $33 \mathrm{GHz}$ and the $92 \mathrm{GHz}$ continuum

\footnotetext{
${ }^{1}$ http://www.cv.nrao.edu/php/splat/
} 
maps have the same resolution, we calculate the spectral index map in this frequency range. This map will help us unveil the nature of the emission in the nuclei of Arp 220. In the top left panel of Figure 4.4 we show such map. We only used the area covered by the $5 \sigma$ contour of the $92 \mathrm{GHz}$ continuum map. From this map we see the nature of the emission is quite different in both nuclei. The most noticeable feature is present in the $\mathrm{W}$ nucleus where we observe a positive spectral index at a position angle (PA) of $\sim-10^{\circ}$. A positive spectral index in this range of frequencies indicates either dust emission contribution or opaque free free emission due to dense ionizing material. The stripe-like morphology of this feature is rather puzzling. A plausible explanation could be the presence of outflowing, highly ionized, warm material, maybe the first direct evidence of an AGN-jet? There is also evidence of $\mathrm{OH}$ maser emission along this North-South direction (Rovilos et al. 2003), which could be generated by either young starbursts or emission from an AGN. We will perform further analysis of this feature in a future work. For the E nucleus the spectral index is mostly flat suggesting thermal emission may dominate in those regions, a likely signature of recent star formation. To further investigate the emission in the nuclei of Arp 220, we constructed a spectral flux density distribution plot at the frequencies covered by the ALMA observations presented in this work. We measured the flux densities of each nuclei and of the whole system, using the images at each SPW, at each sideband, and at $92.2 \mathrm{GHz}$ (see Table 4.1 for the values of integrated flux densities and Section 4.2 for more details on how we obtained these images). The flux densities were obtained by adding pixels with signal greater or equal to $3 \sigma$ within boxes enclosing each component exclusively. We show the final internal-to-Band-3 spectrum in the middle left panel in Figure 4.4. The uncertainties were calculated based on the thermal noise associated to an extended source, and correspond to $\sim 8 \%$ of the measured flux density. From this internal spectrum, we observe the $\mathrm{W}$ nucleus shows a rising behavior, while the $\mathrm{E}$ shows a flatter profile. This is consistent with the spectral index map between 33 and $92 \mathrm{GHz}$. From this internal spectrum we also observe that the shape of the curve for the whole system is controlled by the $\mathrm{W}$ nucleus. This is expected since the $\mathrm{W}$ nucleus is brighter than the $\mathrm{E}$ with a ratio of integrated flux densities of 2:1. These spectra do not reach a minimum, which is expected around these range of frequencies 
(see below). They do show a negative spectral index towards the higher frequency end. This could be due to lower signal-to-noise in the maps and should not be given too much importance. The numbers that can be trusted more are those from the lower and upper sidebands. 

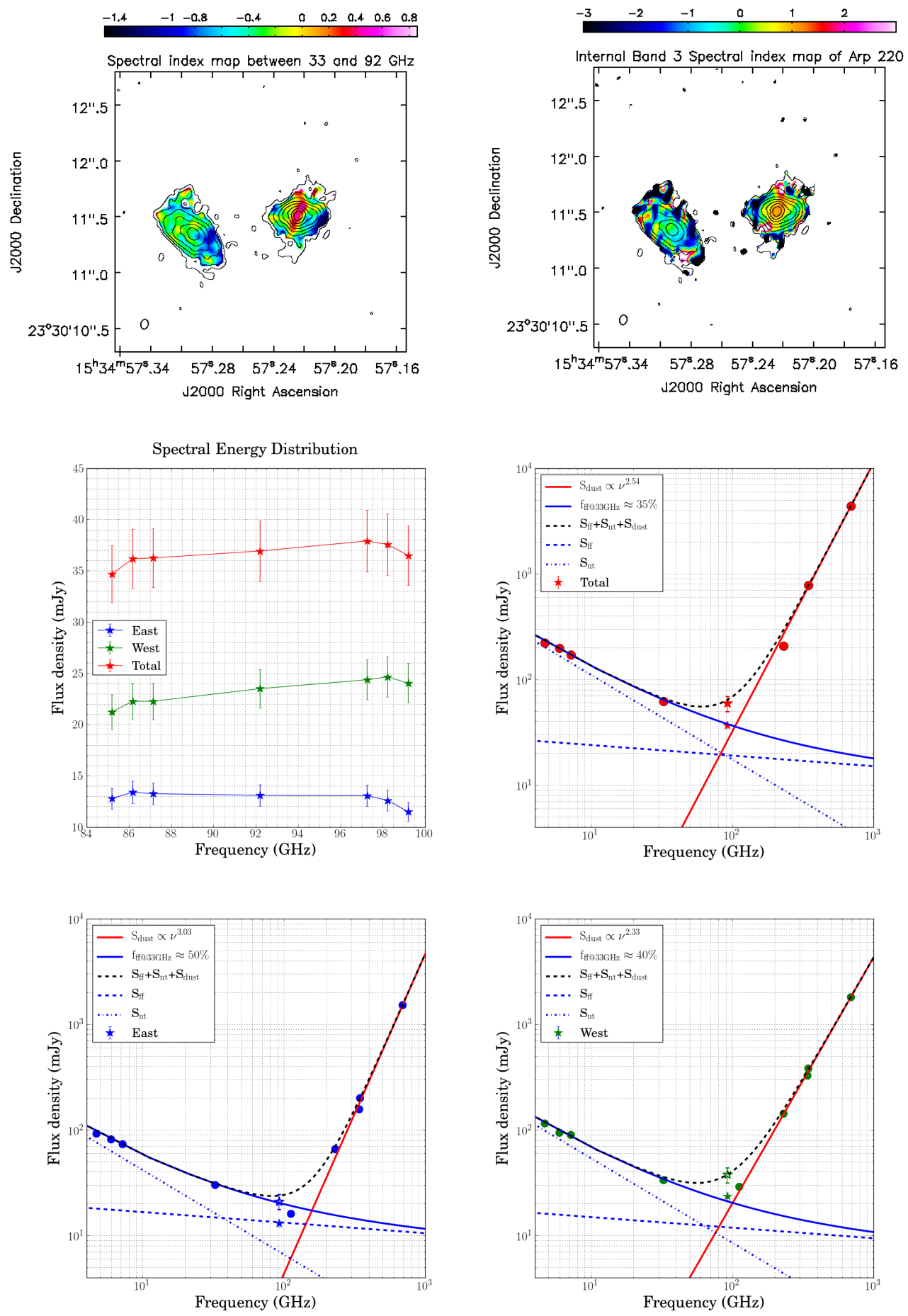

Fig. 4.4.-: (Top Left) Spectral index map between 33 and $92 \mathrm{GHz}$. The contours are those of the $92 \mathrm{GHz}$ continuum map shown in Figure 3.1. (Top Right) Internal Band 3 spectral index map between lower and upper basebands, i.e., between 86.2 and $98.2 \mathrm{GHz}$. The contours in black are the same as the contours in the left panel. (Middle Left) Internal to Band 3 spectral flux density distribution for the entire system (red), western (green) and eastern nucleus (blue). The uncertainties are conservatively assumed to be $8 \%$ of the integrated flux density value. (Middle Right) Spectral flux density distribution of Arp 220 using integrated flux densities from Table 2.2, and only the $92.2 \mathrm{GHz}$ data point from this work. The solid lines (red for dust component, and blue for thermal+non-thermal emission) show the best fit to the data without including the $92.2 \mathrm{GHz}$ data point (star). The blue dashed line is the thermal component and the dotted-dashed blue line is the non thermal component. The black dashed line shows all three components together. The open star symbol is the $92 \mathrm{GHz}$ flux density corrected for the $40 \%$ emission that is potentially missing (see Section 2.2). (Bottom Left) Same as in the previous panel, but for the East component. (Bottom Right) Same as in the previous panel, but for the West component. For details on the model of the spectral flux density distribution see section 4.3 . 
In fact, to study the emission within the ALMA Band 3 range we also calculated a spectral index map between the lower and upper side bands, i.e., between 86.2 and 98.2 GHz (see top left panel in Figure 4.4). From this map, we still see clear differences in the nature of the emission between the two nuclei. The $\mathrm{W}$ nucleus still shows the positive spectral index feature, however the spectral index is now steeper, while the E now shows steep positive spectral indices in one locations and very steep negative spectral indices in other. Overall, the E nucleus shows more negative spectral indices, suggesting more synchrotron dominated emission in those regions. On the opposite, the $\mathrm{W}$ nucleus shows more positive spectral indices.

Finally, we complement our ALMA data with data from the literature (see Table 4.1). We produced a full spectral flux density distribution from the $\mathrm{cm}$ wavelength regime up to sub-mm regime ${ }^{2}$. In the middle-right panel and bottom panels of Figure 4.4, we show the spectra for the entire system and each nuclei. In each case we also show the best fit to the data, excluding the values at $92.2 \mathrm{GHz}$, using a thermal plus a non thermal component, i.e., $\mathrm{S}_{\nu}=\mathrm{S}_{\mathrm{ff}}+\mathrm{S}_{\mathrm{nt}}=\mathrm{A} \times \nu^{-0.1}+\mathrm{B} \times \nu^{-0.8}$, for $\nu<92 \mathrm{GHz}$ and $\mathrm{S}_{\nu}=\mathrm{C} \times \nu^{\gamma}$ for $\nu>92 \mathrm{GHz}$. We found the best fit for the entire source, for $\mathrm{E}$ and $\mathrm{W}$ implied thermal fractions of $35 \%, 50 \%$ and $40 \%$, respectively, at $33 \mathrm{GHz}$. The thermal fraction at $33 \mathrm{GHz}$ of $35 \%$ found for the entire system is consistent with that found by Anantharamaiah et al. (2000) based on radio recombination lines observations. Based on these models, we also found a thermal fraction of $\sim 13 \%$ for the entire system at $6 \mathrm{GHz}$, which is also in agreement with Anantharamaiah et al. (2000). For the dust emission, we found values of $\gamma$ of 2.5, 3.0 and 2.3 for the whole system, the $\mathrm{E}$ and $\mathrm{W}$ nucleus, respectively. These values are consistent with the profile of dust emission.

Note that the modeled spectrum (dashed black line), from the addition of the different components, i.e., $\mathrm{S}_{\mathrm{ff}}+\mathrm{S}_{\mathrm{nt}}+\mathrm{S}_{\text {dust }}$, over predicts the observed flux densities at 92.2 $\mathrm{GHz}$ (solid star symbol) for the east ${ }^{3}$, west, and total cases. This is expected since we only recover $60 \%$ of the total flux density at $92 \mathrm{GHz}$ (see Section 2.2).

\footnotetext{
${ }^{2}$ We only include the values of the integrated flux densities at $92.2 \mathrm{GHz}$ which has the highest signal-to-noise. In a future work, we will also include the lower and upper sideband values.

${ }^{3}$ Note that the value at $112 \mathrm{GHz}$ from Scoville et al. (2016) does not match either the predicted total values, and extended emission is most likely missing due to filtering of large scale emission.
} 
The open star symbol indicates the corrected flux density value accounting for the missing extended emission. In all three cases the corrected value matches well, within the uncertainties, the predicted total flux density at $92 \mathrm{GHz}$ indicated by the black dashed line. The contribution of thermal, non thermal, and dust emission at $92 \mathrm{GHz}$ is $30 \%, 29 \%$, and $41 \%$ for the whole system, $32 \%, 24 \%$, and $44 \%$ for the $\mathrm{W}$ nucleus, and $56 \%, 30 \%$ and $14 \%$ for the E nucleus. The main difference between the nuclei is that the emission in the E nucleus is mostly thermal at $92 \mathrm{GHz}$, while for the $\mathrm{W}$ nucleus is thermal dust.

Overall, a typical spectral flux density distribution constructed with optically thin free-free, synchrotron and dust emission describes well the flux density observed at $92 \mathrm{GHz}$ after correcting for missing extended emission.

\subsection{Line Emission}

The spectra in Figure 4.2 show that we detect our target lines and several others in both nuclei. For most of these, the $\mathrm{W}$ nucleus is stronger than the E. We observe a mixture of strong emission and absorption from transitions of $\mathrm{HCN}, \mathrm{HCO}^{+}, \mathrm{SiO}$, and $\mathrm{C}_{2} \mathrm{H}$, along with transitions of several rarer isotopologues, $\mathrm{H}_{13} \mathrm{CN}$ and $\mathrm{HN}_{13} \mathrm{C}$. We also appear to detect $\mathrm{HC}_{3} \mathrm{~N}$ at the low end of SPW 3, though the whole line is not covered. Several weaker transitions are also evident, these include a firm detection of HNCO, though the line wings may be incompletely covered by SPW 1. In general, less lines are evident and the absorption appears stronger in the central beam, where we integrate over less gas and the continuum is brightest.

The brightest transitions that we observe are $\mathrm{HCN}$ and $\mathrm{HCO}^{+}(1-0)$. These comparatively high dipole moment molecules have correspondingly high critical densities (before accounting for line trapping), $\sim 10^{4} \mathrm{~cm}^{-3}$ for $\mathrm{HCO}^{+}$and $\sim 10^{5} \mathrm{~cm}^{-3}$ for $\mathrm{HCN}$. In observations of more quiescent galaxies, both lines are often used to trace dense gas, in contrast to the CO lines, which trace molecular gas at all densities (e.g., Gao \& Solomon 2004b). Simple arguments suggest that the average density in the Arp 220 nuclei is sufficient to excite emission from both of these lines (e.g, Barcos-Muñoz et al. 2015), and optical depth should be appreciable, lowering the effective density 
needed to excite the lines (e.g., Scoville et al. 2015). As a result, we expect these lines to effectively trace most of the nuclear gas, but to potentially be less contaminated and suffer less opacity from any low density, extended envelope of gas surrounding the nuclei. The rarer isotopologues $\mathrm{H}_{13} \mathrm{CN}$ and $\mathrm{HN}_{13} \mathrm{C}$ have lower abundance but otherwise similar structure to HCN and HNC; thus, we expect them to offer an optically thinner tracer of the gas, with a consequently higher effective critical density. Though isotopic abundances in such an extreme environment are quite uncertain, the contrast between $\mathrm{HCN}$ and $\mathrm{H}^{13} \mathrm{CN}$ or $\mathrm{HC}^{15} \mathrm{~N}$ also offers the prospect to estimate the optical depth of all three lines.

The weaker lines also offer potentially powerful physical diagnostics. SiO is expected to be abundant, and so bright, only when appreciable amounts of silicon have been released from dust grains by strong shocks. HNCO is often taken as a tracer of weaker shocks, $\sim 10-20 \mathrm{~km} \mathrm{~s}^{-1}$ (Meier et al. 2015). $\mathrm{C}_{2} \mathrm{H}$ emission traces photondominated regions (PDRs) and cold, dense gas (e.g., Miettinen 2014). For the most part, we defer analysis of these lines to future work, focusing here on the kinematics observed using the bright lines.

\subsubsection{Absorption and P-Cygni Profiles: Dense Gas Outflow- ing From the Nuclear Disks}

One of the most striking features in the spectra (Figure 4.2 and 4.3) is the prevalence of absorption in many lines across both nuclei. This absorption has been seen in other molecules at high angular resolution (e.g., Sakamoto et al. 2009; Aalto et al. 2015; Rangwala et al. 2015; Scoville et al. 2016). It can be understood as lower temperature gas lying between us and the bright continuum that is absorbing a portion of the continuum emission. The gas has a lower temperature (and so a fainter source function) than the brightness temperature of the continuum, leading to absorption. These features are more prominent in the single-beam nuclear spectra, where the continuum reaches its maximum. These absorption features are strongest in HCN and $\mathrm{HCO}^{+}$, but also visible in $\mathrm{SiO}$, (in $\mathrm{E}$ ) $\mathrm{H}^{13} \mathrm{CN}$.

In the eastern nucleus, the lines share a common shape. The line is absorbed 
at velocities lower ("blue") than systemic, and the line shows emission at velocities higher ("red") than systemic. This shape resembles the classic "P-Cygni" profile generated by an expanding shell of gas around a bright central continuum source. In the P-Cygni profile, red-shifted emission comes from gas that is expanding away from the observer, on the far side of the source. The blue-shifted absorption of the profile comes from the part of the shell between us and the central bright source. This intervening material is expanding away from the source, coming towards us, and is thus blue-shifted. This feature appears in absorption because it lies between us and the background continuum source, and because the gas in the shell has a lower brightness temperature than the central continuum source, as we mentioned above.

The P-Cygni profile is clearest in the spectra of the central beam (see Figure 4.3 ), and in the E most of the strong spectral lines show this profile in the nuclear spectrum. We compile these profiles into a single plot for each nucleus in Figure 4.5.
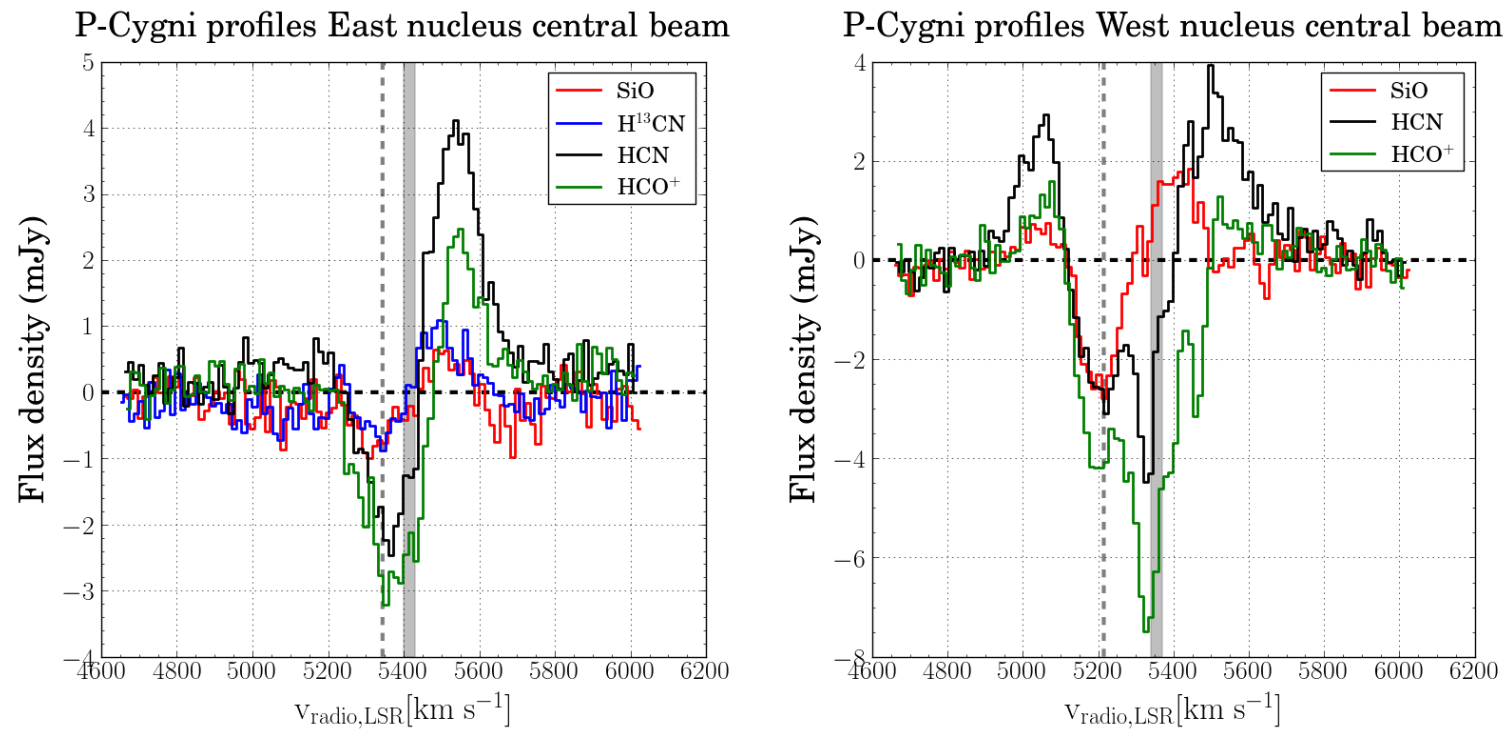

Fig. 4.5.- : P-Cygni profiles from the central beam of the East (Left) and West (Right) nucleus of Arp 220. The spectral resolution of these profiles is $13.6 \mathrm{~km} \mathrm{~s}^{-1}$. For the east nucleus, the P-Cygni profile is evident for $\mathrm{HCO}^{+}$ (green), $\mathrm{H}^{13} \mathrm{CN}$ (blue), $\mathrm{HCN}$ (black) and $\mathrm{SiO}$ (red) molecular lines, while for the west nucleus is evident only for $\mathrm{HCN}$ and $\mathrm{SiO}$. The solid grey band shows the systemic velocity for each nucleus. The dashed grey line shows a characteristic blue shifted velocity for the absorption feature in the P-Cygni profile, which is $\sim 70 \mathrm{~km} \mathrm{~s}^{-1}$ from vsys $(\mathrm{E})$ in the eastern nucleus and $\sim 140 \mathrm{~km} \mathrm{~s}^{-1}$ from $\mathrm{v}_{s y s}(\mathrm{~W})$ in the western nucleus.

The West shows a slightly more complex pattern. There is blue-shifted absorption but emission re-emerges at very low (blue) velocities, and the lines show distinct 
profiles. There are several possible explanations here. Perhaps the spectrum is a mixed of inflow and outflow features, so that some blue-shifted emission comes from behind the source. Alternatively, the continuum source may be confined within the beam, so that the extended emission corresponds to rotating gas, with only a subset of the gas, including some outflowing material, between us and the source. There appears likely to be some outflow in the W, because strong absorption is seen blueshifted without a corresponding red-shifted absorption component. Perhaps most striking in the $\mathrm{W}$ profile, $\mathrm{SiO}$ appears to produce a cleaner P-Cygni profile than HCN or $\mathrm{HCO}^{+} . \mathrm{HCO}^{+}$goes almost totally into absorption at a range of velocities, as one might expect for a rotating disk partially in front of a continuum source. Perhaps the $\mathrm{SiO}$ emission highlights shocked, outflowing gas while the more general gas tracers also capture a large rotating component. Another explanation for the $\mathrm{HCO}^{+}$may be self-absorption by foreground $\mathrm{HCO}^{+}$emission as proposed by Aalto et al. (2015).

We use the profiles to estimate outflow velocities and mass outflow rates, which are more uncertain in the $\mathrm{W}$ due to the ambiguous nature of the line profile. We begin by assuming a shell-like geometry. In this case, the blue-shifted feature corresponds to gas coming directly towards us, and the maximum expansion velocity of the shell can be directly measured from the minimum of the absorption feature. The strongest absorption is displaced $70 \mathrm{~km} \mathrm{~s}^{-1}$ from systemic in E and $140 \mathrm{~km} \mathrm{~s}^{-1}$ from systemic in $\mathrm{W}$. We highlight these velocities with dashed lines in Figure 4.5. For the simplest possible geometry, these would be the outflow velocity of the shells. These velocities are in good agreement with the $100 \mathrm{~km} \mathrm{~s}^{-1}$ outflow velocities found by Sakamoto et al. (2009) based on P-Cygni profiles seen from CO (3-2) spectra that marginally resolved each nuclei.

These velocities are pure line-of-sight velocities and assume a symmetric spherical expansion. They will underestimate the true outflow velocity if the outflow is not a sphere but instead an inclined cone or jet, which would still show blue-shifted absorption against a moderately extended background continuum source. In this case, if the outflow has an inclination $i$ with respect to the line of sight, then the velocity of the outflow would be higher by a factor of $1 / \cos (\mathrm{i})$. Galactic winds are often launched perpendicular to the disk (e.g., see Veilleux et al. 2005), so that the 
inclination of the outflow will be offset by $90^{\circ}$ from that of the galaxy. If we make this assumption then for our adopted disk geometries (following the model in BarcosMuñoz et al. (2015)), the outflow would have $i \sim 32^{\circ}$ for $\mathrm{E}$ and $36^{\circ}$ for $\mathrm{W}$, implying correction factors by up to 1.18 and 1.24 if the outflow emerges perpendicular to the apparent disks. In this case, the true outflow velocities would be $\sim 80(\mathrm{E})$ and $170 \mathrm{~km} \mathrm{~s}^{-1}$.

Combining this outflow velocity with an amount of mass participating in the outflow and a size scale, we can estimate a mass outflow rate. All of these are uncertain, of course, but attempting this calculation yields an idea of the importance of the outflow to the fate of the nuclear starbursts. We can, in principle, estimate the mass of outflowing material in two ways: we could measure the optical depth of absorption, translate it to a column density of molecules, and convert this to a molecular gas mass using an assumed abundance. Alternatively, we can assume that the red-shifted emission is all participating in the outflow and combine this emission with an assumed mass-to-light ratio. In either case, we would assume symmetry and double the resulting estimate. Here, we adopt the latter approach, integrating the red side of the spectrum and applying an HCN-to-dense-gas conversion factor $\alpha_{\mathrm{HCN}} \sim 10 \mathrm{M}_{\odot}\left(\mathrm{K} \mathrm{km} \mathrm{s}^{-1} \mathrm{pc}^{2}\right)^{-1}($ Gao \& Solomon 2004a). This adopted mass to light ratio is very uncertain, as it combines an adopted abundance, density distribution, opacity, and physical conditions. Still, the calculation should yield a useful indication of the outflow rate.

We adopt a filled cone geometry, which is commonly used in studies of molecular outflows (e.g., Cicone et al. 2014). In this case

$$
\dot{M}_{\mathrm{OF}}=3 v \frac{M_{\mathrm{OF}}}{R_{\mathrm{OF}}},
$$

where $v$ is the outflow velocity, $M_{\mathrm{OF}}$ is the mass participating in the outflow, and $R_{\mathrm{OF}}$ is the extent of the outflow. Lacking a clean constraint on $R_{\mathrm{OF}}$, we adopt the size of our beam (45 pc) as the characteristic scale for measurements from the nuclear spectrum.

Using HCN, we estimate masses associated with the outflow of $\sim 3 \times 10^{8} M_{\odot}$ for 
both nuclei, this value is uncertain by at least a factor of a few. Including the inclination correction for $v$, these imply mass outflow rates of $1600(\mathrm{E})$ and $3400 \mathrm{M}_{\odot} \mathrm{yr}^{-1}$ (W). These mass outflow rates are 18 and 35 times higher than the star formation rate of each nucleus, $\sim 90(\mathrm{E}) \sim 100(W) \mathrm{M}_{\odot} \mathrm{yr}^{-1}$ (based on $33 \mathrm{GHz}$ emission from Barcos-Muñoz et al. 2015). Such high mass loading factors $\left(\dot{M}_{\mathrm{OF}} / \mathrm{SFR}\right)$ have been seen mostly in systems hosting AGN, suggesting their presence helps boost the outflow rate (Cicone et al. 2014). The fact that the dense molecular gas is being removed at a higher rate than the formation of stars suggest star formation feedback and potential future quenching of the system. There is a strong caveat regarding these high mass outflow rates and that is they strongly depend on the conversion factor. These numbers should only be taken as conservative upper limits.

\subsubsection{Kinematics From $\mathrm{HCN}$ and $\mathrm{HCO}^{+}$Emission}

A main motivation for our observations was to use the kinematics and distribution of the gas to model the mass and structure of the two nuclear disks. $\mathrm{HCN}$ and $\mathrm{HCO}^{+}$ are two of the most well studied molecules in extragalactic sources. Eventually, we plan to carry out such modeling combining both optically thin (fainter) lines and the bright $\mathrm{HCN}$ and $\mathrm{HCO}^{+}$lines. For this first analysis, we focus on only the two bright transitions.

Figure 4.6 and 4.7 show the integrated intensity, mean velocity, and second moment (velocity dispersion) of $\mathrm{HCN}$ and $\mathrm{HCO}^{+}$. We resolve both disks in both lines. HCN emission appears slightly more extended than the continuum emission and covers the entire extent of both nuclei. $\mathrm{HCO}^{+}$emission also extends across both disks, but is more patchy, at least in part due to the presence of more absorption in both nuclei. Overall $\mathrm{HCO}^{+}$emission is weaker than $\mathrm{HCN}$, perhaps due to opacity effects or perhaps simply due to a lower abundance of the $\mathrm{HCO}^{+}$molecule. 

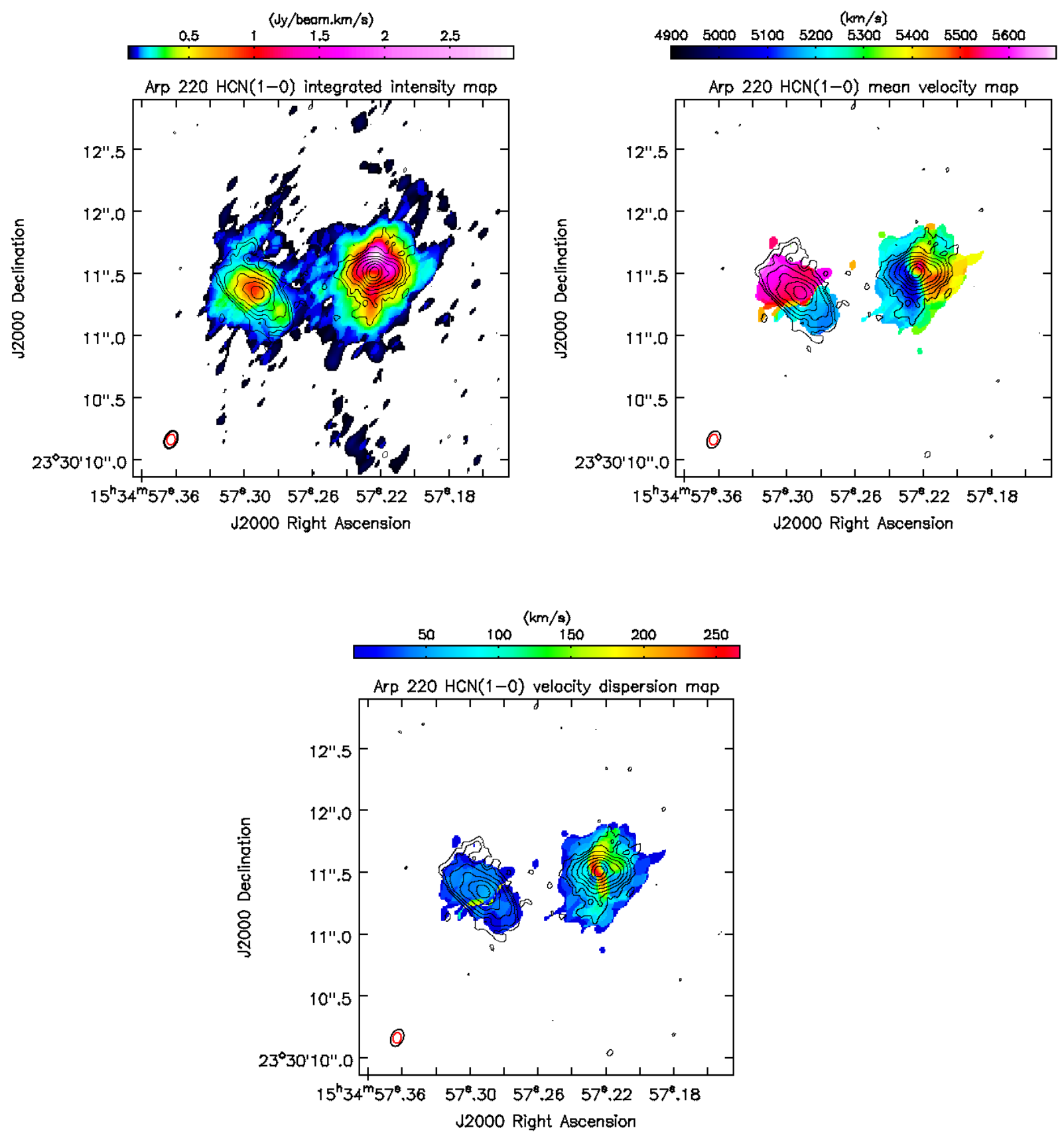

Fig. 4.6.- : HCN (1-0) moment maps of Arp 220 with the $92 \mathrm{GHz}$ continuum contours from Figure 4.1 overlaid. The beam size of the HCN cube $\left(0^{\prime \prime} 15 \times 00^{\prime \prime} 10\right)$ and the continuum emission are shown in the bottom left corner of each panel in black and red. Integrated intensity map or zeroth moment map (Top - Left), mean velocity or first moment map (Top - right), and velocity dispersion map or second moment (Bottom) of Arp 220. In each map, the beam is shown in black in the bottom left corner. The zeroth moment map was masked at $3 \sigma$, with $\sigma=0.2$ mJy beam ${ }^{-1} \mathrm{~km}$ $\mathrm{s}^{-1}$. The other two maps were masked at $5 \sigma$. 

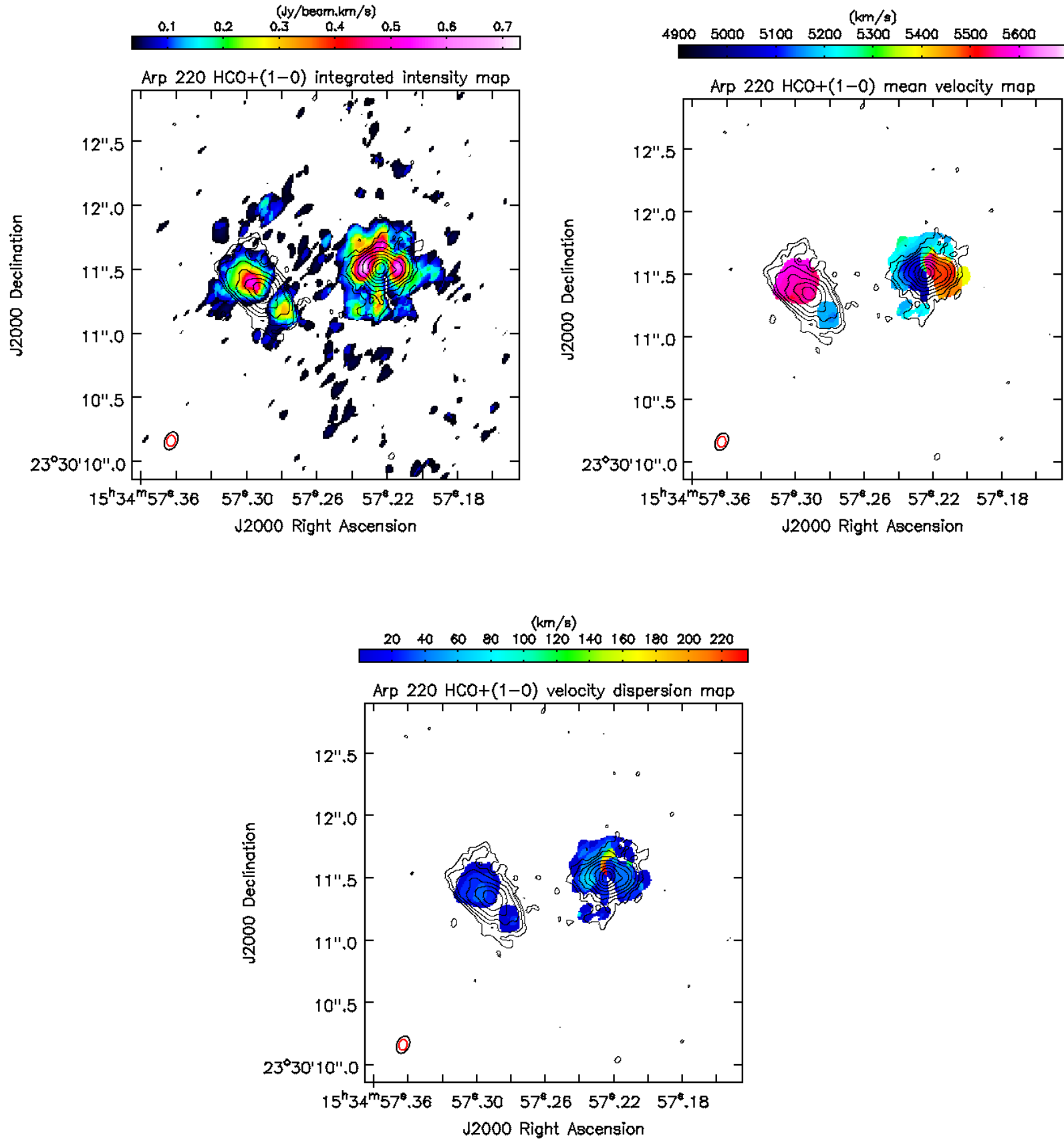

Fig. 4.7.- $: \mathrm{HCO}^{+}(1-0)$ moment maps of Arp 220 with the $92 \mathrm{GHz}$ continuum contours from Figure 4.1 overlaid. The beam size of the $\mathrm{HCO}^{+}$cube $\left(0^{\prime \prime} 15 \times 00^{\prime \prime} 10\right)$ and the continuum emission are shown in the bottom left corner of each panel in black and red. Integrated intensity map or zeroth moment map (Top - Left), mean velocity or first moment map (Top - right), and velocity dispersion map or second moment (Bottom) of Arp 220. In each map, the beam is shown in black in the bottom left corner. The zeroth moment map was masked at $3 \sigma$, with $\sigma=0.2 \mathrm{mJy}$ beam ${ }^{-1} \mathrm{~km} \mathrm{~s}^{-1}$. The other two maps were masked at $5 \sigma$. 
For the $\mathrm{W}$ nucleus, the location of the peak of the HCN emission do not coincide with that of the $\mathrm{HCO}^{+}$. For the E nucleus, the peaks of both tracers are closer, but do not match. The differences in the morphology and peaks of both tracers indicates the ISM conditions are different in each nucleus. These could be due to the different optical depths, different densities of the gas each molecule is tracing, the abundance of each molecule and radiative ionization effects.

From the moment 1 maps, we clearly observe the gas is rotating. In the $\mathrm{W}$ nucleus, the dense gas is rotating such that the east side of the disk is blue-shifted (coming towards us) and the west side is red-shifted (going away from us). This pattern indicates the kinematic axis around which the gas is moving is along the north-south direction, almost perpendicular to its major axis. For the E nucleus, the south west part of the disk is blue-shifted, while the north east part of the disk is red-shifted. The kinematic axis is then perpendicular to the major axis of the disk $\left(\mathrm{PA} \approx 56^{\circ}\right)$. This is consistent with what Sakamoto et al. (1999) derived for the Arp 220 nuclei using the $\mathrm{CO}(2-1)$ line emission, and it is shown in their schematic illustration in their Figure 5.

Rotation is also evident from the position-velocity $(\mathrm{P}-\mathrm{V})$ diagrams shown in Figure 4.8. We created P-V diagrams along the major axis of each nucleus for the HCN and $\mathrm{HCO}^{+}$emission. The position angles we used, $56^{\circ}$ and $80^{\circ}$ for $\mathrm{E}$ and $\mathrm{W}$, were those derived from the $33 \mathrm{GHz}$ continuum maps (see Barcos-Muñoz et al. 2015, for further details). 

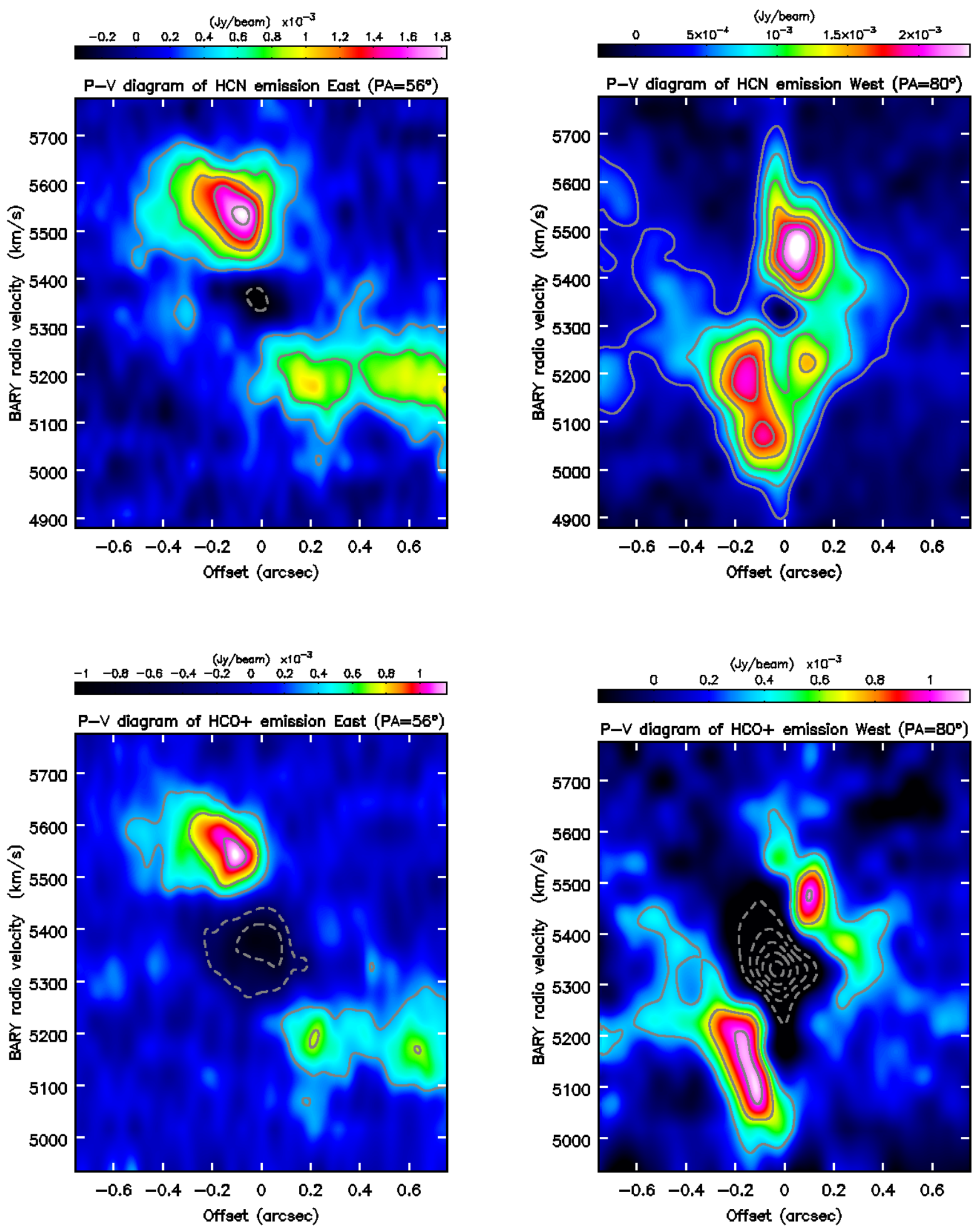

Fig. 4.8.- : Position velocity (P-V) diagrams for E (left panels) and W (right panels) nuclei for the HCN (1-0) (top panels) and $\mathrm{HCO}^{+}(1-0)$ (bottom panels) line emission. These diagrams were obtained from a slice along the major axis of each nucleus, at a position angle of $56^{\circ}$ and $80^{\circ}$ for $\mathrm{E}$ and $\mathrm{W}$, respectively. The width of the slice is $1^{\prime \prime}$ so we obtain high signal-to-noise. The contours are in steps of $\pm 1.5 \sigma, \pm 3 \sigma, \pm 4.5 \sigma, \ldots$, where $\sigma=0.2 \mathrm{mJy} \mathrm{beam}{ }^{-1} \mathrm{~km} \mathrm{~s}^{-1}$ and negative contours are dashed. At the distance of Arp 220, $1^{\prime \prime}=369$ pc. The beam size is $00^{\prime \prime} 15 \times 0$.' 1 . 
From these $\mathrm{P}-\mathrm{V}$ diagrams we observed that for $\mathrm{HCN}$ and $\mathrm{HCO}^{+}$, the $\mathrm{E}$ nucleus shows components moving as fast as $235 \mathrm{~km} \mathrm{~s}^{-1}$ at 0.23 from the center of the eastern nucleus. If we assume the mass is distributed in a spherical geometry and is following a circular motion, we can estimate the dynamical mass enclosed within a certain radius $\left(\mathrm{M}(<\mathrm{R})=\mathrm{R} \mathrm{V}^{2} / \mathrm{G}\right.$, where $\mathrm{R}$ is the radius at which the dynamical mass is calculated, $\mathrm{V}$ the velocity at that radius and $\mathrm{G}$ is the gravitational constant). We estimate the dynamical mass within the central 110 pc of the E nucleus is $\sim 1.42 \times 10^{9} \mathrm{M}_{\odot}$.

For the $\mathrm{W}$ nucleus, we find components moving as fast as $305 \mathrm{~km} \mathrm{~s}^{-1}$ at $0{ }^{\prime \prime} 07$ from the center of the western nucleus. We estimate the dynamical mass within the central $26 \mathrm{pc}$ of the $\mathrm{W}$ nucleus is $\sim 5.6 \times 10^{8} \mathrm{M}_{\odot}$.

\subsection{Conclusions}

We make use of continuum and line emission at $\sim 0^{\prime \prime} 1$ spatial resolution to address the nature of the energy source at $3.3 \mathrm{~mm}$, as well as the gas kinematics and gas mass radial profiles of the nuclei of Arp 220.

From the continuum emission we find that the western nucleus contributes $2 / 3$ of the total flux density of the system at $92 \mathrm{GHz}$ and it dominates the spectral flux density distribution shape of the whole system. We also find that within the range of frequencies covered by our observations, both nuclei show different behaviors with the western nucleus showing a rising spectral index through the high frequency end. This indicates contribution from opaque free-free or dust emission. The eastern nucleus shows a flatter spectrum implying free-free emission is still an important component at this high frequencies. In fact, when we combine the flux density measured at $92 \mathrm{GHz}$ with data at other frequencies from the literature, we find the continuum emission is well described by a combination of optically thin free-free, synchrotron and thermal dust emission. The western nucleus is dominated by thermal dust emission (44\%) at $92 \mathrm{GHz}$, while the eastern nucleus is dominated by free-free emission (56\%).

From the line emission, we detect several dense gas tracers including $\mathrm{HCN}, \mathrm{HCO}^{+}$, and their isotopologues. We also detect $\mathrm{SiO}, \mathrm{C}_{2} \mathrm{H}$ and $\mathrm{H}^{3} \mathrm{CN}$. From the central beam spectra we observe P-Cygni profiles in both nuclei indicating the presence of outflows. 
The eastern profiles show clear red-shifted emission and blue-shifted absorption, while the western nucleus shows deep and complex absorption features, and blue-shifted emission. From these P-Cygni features we derived maximum outflow velocities, corrected for inclination, of $170(\mathrm{~W})$ and $80 \mathrm{~km} \mathrm{~s}^{-1}(\mathrm{E})$. The derived mass outflow rates are 18 and 35 times larger than the SFR of each nuclei. Such high mass loading factors has been mostly detected in sources hosting AGN which helps boost the outflow rate. This suggests the potential presence of AGN in the nuclei of Arp 220.

We also study the kinematics of the dense gas as traced by $\mathrm{HCN}$ and $\mathrm{HCO}^{+}$. From the moment 0 maps we find $\mathrm{HCN}$ is slightly more extended than the continuum emission at $92 \mathrm{GHz}$, while $\mathrm{HCO}^{+}$is less abundant indicating a difference in the gas traced by either line. These differences may be due to abundance, chemistry, UV-radiation, etc. We also find that the dense gas in both nuclei is rotating. The derived dynamical masses are $1.42 \times 10^{9} \mathrm{M}_{\odot}$ within $110 \mathrm{pc}$ for the eastern nucleus, and $5.6 \times 10^{8} \mathrm{M}_{\odot}$ within $26 \mathrm{pc}$ for the western nucleus.

\subsection{Future Work}

There is still further analysis to be done to take full advantage of this rich data set. To study potential extended emission around the nuclei we intend to image and analyze the emission at different scales. Note that the maximum recoverable scale is 1 .'1, so features bigger than this will be resolved out.

From the continuum point of view, we still have not included low frequency information (e.g., Varenius et al. 2016, for high resolution observations at $150 \mathrm{MHz}$ ) that will help us constrain how much free-free absorption is present in each nucleus. In addition, lower resolution observations at $92 \mathrm{GHz}$ will help us determine exactly how much extended emission is missing and the nature of such emission.

Regarding the kinematics of the system, we will model the emission line cubes (together and separately) to fit the velocity dispersion and rotation velocity as a function of radius in each disk, which has been attempted in slightly resolved velocity fields but with limited success (Scoville et al. 2015). These detailed kinematics will yield improved dynamical masses and mass profiles compared to (Engel et al. 2011) 
or the sub-mm studies mentioned in the introduction. Ideally, we aim to obtain the mass decomposition for each nucleus, i.e., to calculate the gas, dynamical, black hole and stellar masses for each disk. From the velocity dispersion profiles we will be able to estimate diskiness and scale heights of the two nuclei and so work towards a much-needed three dimensional picture of the two nuclei. This is essential to compare Arp 220, a key test case, for models considering star formation in extreme disks and different modes of feedback (Thompson et al. 2005; Ostriker \& Shetty 2011).

Methodologically, in addition to the basic moment analysis shown here, we have experience testing models of disk structure (in Arp 220, Barcos-Muñoz et al. 2015) and directly modeling the 3-D emission in a cube taking into account finite resolution and a model intensity distribution (see Leroy et al. 2015, applied to NGC 253 following the methodology of Davis et al. (2013)). This Davis et al. methodology was developed to constrain black hole masses in marginally resolved mm emission line cubes and will be ideal to jointly model the gas distribution plus kinematics in Arp 220. The result should be a substantially tightened dynamical constraint on the role of a black hole in the western nucleus, where a potentially Compton thick active galactic nucleus (AGN) has long been argued (e.g., Downes \& Eckart 2007). For our 30 pc resolution and assuming a velocity dispersion of $200 \mathrm{~km} \mathrm{~s}^{-1}$, we would be able to probe the sphere of influence for a Black hole mass of $3 \times 10^{8} \mathrm{M}_{\odot}$.

\section{Acknowledgments}

L.B-M. was supported by NRAO through the Grote Reber predoctoral fellwoship. This research made use of the NASA/IPAC Extragalactic Database (NED), which is operated by the Jet Propulsion Laboratory, California Institute of Technology, under contract with the National Aeronautics and Space Administration, and NASA's Astrophysics Data System Bibliographic Services. The National Radio Astronomy Observatory is a facility of the National Science Foundation operated under cooperative agreement by Associated Universities, Inc. This paper makes use of the following ALMA data: ADS/JAO.ALMA\#2015.1.00702.S. ALMA is a partnership of ESO (representing its member states), NSF (USA) and NINS (Japan), together with NRC 
(Canada), NSC and ASIAA (Taiwan), and KASI (Republic of Korea), in cooperation with the Republic of Chile. The Joint ALMA Observatory is operated by ESO, AUI/NRAO and NAOJ. 


\section{Chapter 5}

\section{Summary and Future Work}

\section{$5.1 \quad$ Summary}

This thesis focuses on the study of star formation in local U/LIRGs from a high resolution radio continuum perspective. In Chapter 2, 6 and $33 \mathrm{GHz}$ high spatial resolution continuum emission from the closest, prototypical ULIRG, Arp 220, are analyzed. The $33 \mathrm{GHz}$ thermal emission is lower than expected based on infrared observations, which indicates there is a potential suppression of free-free electrons by surrounding dust. The two nuclei in Arp 220 are well resolved at $33 \mathrm{GHz}$, with measured half-life radii of 51 (eastern nucleus) and 35 (western nucleus) pc. Further, the estimated SFR and gas surface densities of the nuclei place them among the most extreme star-forming regions ever observed for any galaxy. However, both nuclei lie well below the Eddington-limit of "maximal starburst". Future studies of the gas conditions is necessary to determine the true Eddington limit of each nucleus.

In Chapter 3, the radio data analysis is expanded to all 22 U/LIRGs in the sample, with a focus on the high resolution (i.e., $33 \mathrm{GHz}$ ) part of this radio survey. From these observations, it is observed that the deconvolved half-light sizes of the energetically dominant regions in the sample range from 20 to $1.7 \mathrm{kpc}$. The emission at 33 $\mathrm{GHz}$ is mostly an equal mixture of free-free and synchrotron emission, making the sizes measured suitable to describe the extent of star forming and/or AGN dominant regions. The SFR and gas densities derived using these measured sizes place these 
systems among the most extreme extragalactic environments ever studied. The star formation-gas scaling relation is used to compare the U/LIRGs with regions within normal spiral galaxies. The presence of two modes of star formation is inferred in the comparison, although this result is extremely dependent on the CO-to- $\mathrm{H}_{2}$ conversion factor. From the values of the star formation rate surface densities, the [CII] deficit increases with increasing $\Sigma_{\mathrm{SFR}}$, increasing opacity and decreasing size. This agrees with previous findings of [CII] deficit being more pronounced in the most compact and obscured U/LIRGs. Even though these systems show one of the highest infrared surface densities ever measured, most of the targets are not producing stars at the maximum limit set by radiation pressure on dust.

Finally, in Chapter 4, ALMA observations of dense molecular gas tracers and $3.3 \mathrm{~mm}$ continuum emission from Arp 220 are presented. The level of the $92 \mathrm{GHz}$ continuum emission is well described by a combination of optically thin free-free, synchrotron and thermal dust emission. At $3.3 \mathrm{~mm}$ the western nucleus is dominated by thermal dust emission while the eastern nucleus is dominated by free-free emission. Several dense gas tracers including $\mathrm{HCN} \mathrm{HCO}^{+}$and their isotopologues, as well as the shock tracer $\mathrm{SiO}$, are detected. The gas is shown to be rotating and well distributed between the two nuclei. The $\mathrm{HCO}^{+}$emission is weaker than $\mathrm{HCN}$ in part due to

opacity effects, but also potentially due to abundance differences. $\mathrm{HCO}^{+}$is also subject to stronger absorption towards the center of each nucleus. Further, there is evidence of outflows in both nuclei, with derived mass loading factors of 18 and 35 for the eastern and western nuclei, respectively. This suggests an AGN may be boosting the observed mass outflow rates, however these numbers are highly uncertain due to the assumed HCN-to-gas mass conversion factor.

\subsection{Future Work}

\subsubsection{Detail spectral index maps}

All of the radio galaxy data obtained with the VLA survey were not presented in this thesis. A dataset comparable to that presented for Arp 220 in Chapter 2 exists for the 


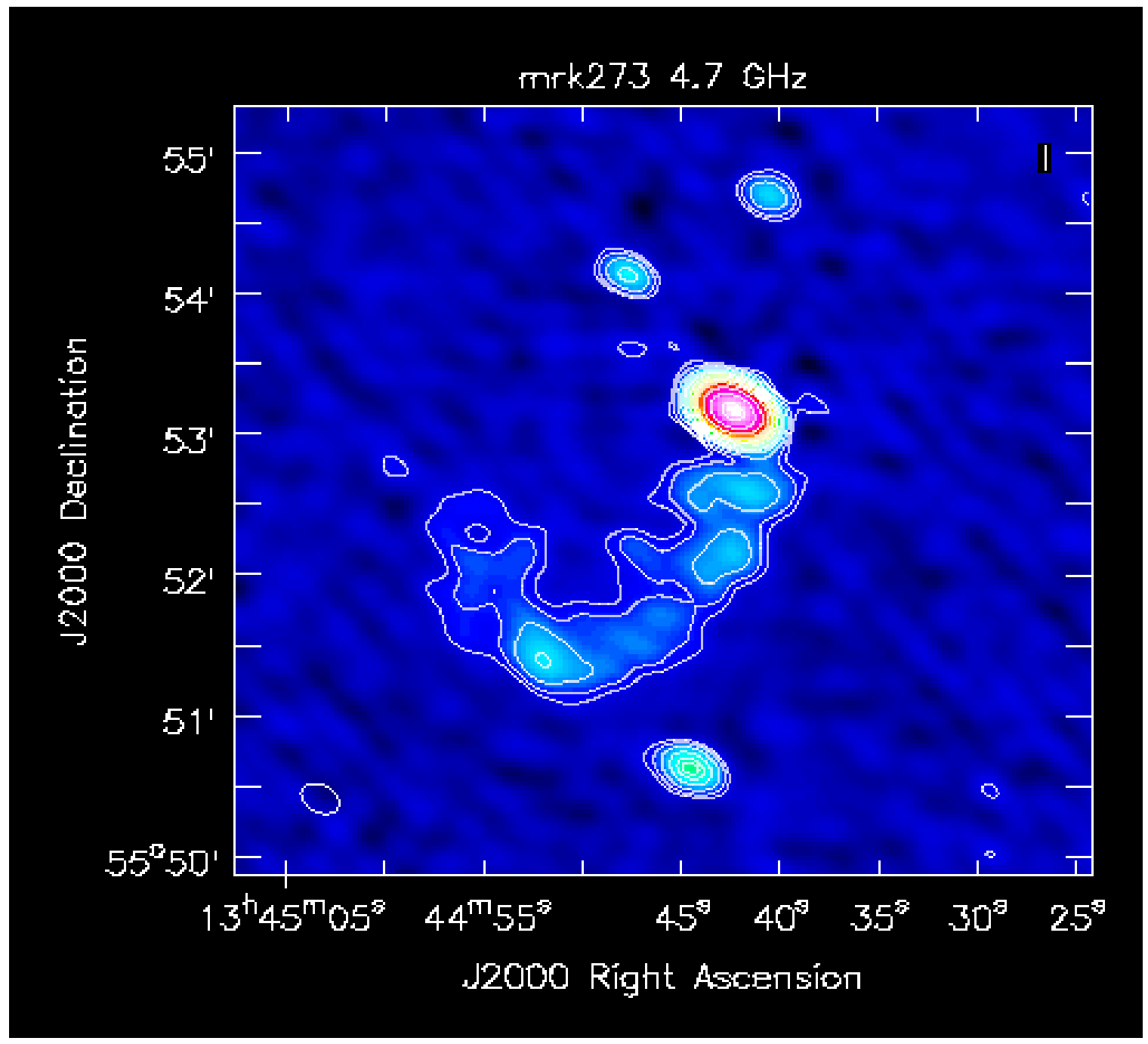

Fig. 5.1.- : Preliminary results on the extended continuum emission seen at $4.7 \mathrm{GHz}$ in the local merger UGC 08696 (Mrk 273). This is data obtained with the VLA D configuration (synthesized beam size is $17^{\prime \prime} \cdot 5 \times 11^{\prime \prime} 7$. The contours are in steps of $2^{n} \times 3 \sigma$, with $\mathrm{n}=1,2,3, \ldots$ and $\sigma=25 \mu \mathrm{Jy}$ beam $^{-1}$. Signal to noise of $\sim 2680$ in 5 minutes of integration time.

entire sample of $22 \mathrm{U} /$ LIRGs. Thus, a future project is the analysis and publication of the low frequency $(6 \mathrm{GHz})$ part of the radio survey. I will obtain detailed spectral index maps between 6 and $33 \mathrm{GHz}$ for the whole sample. In addition, preliminary work on this data show extended synchrotron emission in some galaxies (see Figure 5.1 for an example on extended emission in UGC 08696). I will report these findings and if possible compare this extended emission to observations at other wavelengths in order to understand the potential origin of such features. 


\subsubsection{Radio Recombination Lines in Arp 220}

Radio recombination lines (RRLs) provide a powerful tool for extracting crucial information of the physical conditions of the thermal gas, including its kinematics, electron temperature, electron density and the geometry of ionized gas distribution. I have led a successful VLA proposal (20 hours of awarded time, priority B) to study the kinematic properties of the disks of Arp 220, through the use of Radio Recombination Lines (RRLs). The plan will be to complement results and data from Chapters 2 and 4 with these observations to get a full picture of the gas kinematics and star formation distribution in the nuclei of Arp 220.

\subsubsection{Probing Radio Physics in Detail}

I have also led a successful proposal ( 5 hours of VLA time) to observe the local merger II Zw 096 at different frequency bands covering the range 1 to $50 \mathrm{GHz}$ available at the VLA. This galaxy shows a variety of environments within its extent, including a spiral galaxy, an off-nuclear compact starburst, and more diffuse star-forming region. For each region, I will measure the synchrotron slope, the thermal fraction, and high frequency breaks or low frequency signatures of opacity in many resolution elements. The quantities will be derived as a function of the location within the merger (e.g., distance from the nearest star-forming region or nucleus,) which will aid in constraining the origin of the emission. From the thermal emission, I will measure the true distribution of star formation, and calibrate the use of monochromatic radio emission as a star formation indicator. Finally, via comparisons to in-hand ALMA (Stierwalt et al. in prep) CO mapping, I will measure the (heavily) resolved star formation-gas relation in these merging systems. The VLA provides the opportunity to disentangle this complexity due to its ability to rapidly observe $\mathrm{RC}$ from systems across decades in frequencies and out to high redshift. I am also involved on a Very Long Baseline Interferometry (VLBI) project (PI: Stierwalt) to observe continuum emission from the off-nuclear starburst in II Zw 096 mentioned above. The goal of the project is to determine whether this off-nuclear source is really a starburst (a group of SNR, like those seen in Arp 220), and/or a powerful AGN. 


\subsubsection{Gas content and kinematics in local U/LIRGs}

Measuring the detailed structure, inner dynamics and gas content of these local $\mathrm{U} /$ LIRGs is crucial to understanding the underlying physics of the extreme regime of star and galaxy formation. These types of measurements have been very difficult to make - local U/LIRGs are rare, compact and relatively far away - until now. In the future, I will concentrate my efforts on using the high spatial resolution and sensitivity of ALMA, the VLA, and VLBI to observe the gas content and continuum emission of local U/LIRGs in unprecedented detail. I will use these observations to: 1) determine whether these systems are "maximum-rate starbursts" (limit set by radiation pressure on dust as the main feedback mechanism), 2) better constrain the CO-to- $\mathrm{H}_{2}$ conversion factor for starbursts, 3) study the star formation law for extreme starburst at few $\sim 10$ pc scales, 4) study molecular outflows properties, if present, 5) constrain the masses, and existence, of the supermassive black holes in the nuclei of these systems, and 6) determine the true star formation extent.

These topics of feedback, measuring the properties of the molecular gas, and studying the modes of star formation have a wide relevance to star and galaxy formation at low and high redshift. In all cases, the key to understanding the physics governing these extreme environments is resolving the structure of the inner disks of these deeply embedded, compact galaxies. The outstanding sensitivity and resolution power of ALMA, combined with the high spatial resolution and frequency range covered by the Jansky VLA, allow for the measurement of resolved kinematics and the gas structure of these systems with a detail never achieved before. 


\section{References}

Aalto, S., Martín, S., Costagliola, F., González-Alfonso, E., Muller, S., Sakamoto, K., Fuller, G. A., García-Burillo, S., van der Werf, P., Neri, R., Spaans, M., Combes, F., Viti, S., Mühle, S., Armus, L., Evans, A., Sturm, E., Cernicharo, J., Henkel, C., \& Greve, T. R. 2015, A\&A, 584, A42

Ali-Haïmoud, Y., Hirata, C. M., \& Dickinson, C. 2009, MNRAS, 395, 1055

Alonso-Herrero, A., Rieke, G. H., Rieke, M. J., \& Scoville, N. Z. 2000, ApJ, 532, 845

Anantharamaiah, K. R., Viallefond, F., Mohan, N. R., Goss, W. M., \& Zhao, J. H. 2000, ApJ, 537, 613

Armus, L., Charmandaris, V., Bernard-Salas, J., Spoon, H. W. W., Marshall, J. A., Higdon, S. J. U., Desai, V., Teplitz, H. I., Hao, L., Devost, D., Brandl, B. R., Wu, Y., Sloan, G. C., Soifer, B. T., Houck, J. R., \& Herter, T. L. 2007, ApJ, 656, 148

Armus, L., Mazzarella, J. M., Evans, A. S., Surace, J. A., Sanders, D. B., Iwasawa, K., Frayer, D. T., Howell, J. H., Chan, B., Petric, A., Vavilkin, T., Kim, D. C., Haan, S., Inami, H., Murphy, E. J., Appleton, P. N., Barnes, J. E., Bothun, G., Bridge, C. R., Charmandaris, V., Jensen, J. B., Kewley, L. J., Lord, S., Madore, B. F., Marshall, J. A., Melbourne, J. E., Rich, J., Satyapal, S., Schulz, B., Spoon, H. W. W., Sturm, E., U, V., Veilleux, S., \& Xu, K. 2009, PASP, 121, 559

Barcos-Muñoz, L., Leroy, A. K., Evans, A. S., Privon, G. C., Armus, L., Condon, J., Mazzarella, J. M., Meier, D. S., Momjian, E., Murphy, E. J., Ott, J., Reichardt, A., Sakamoto, K., Sanders, D. B., Schinnerer, E., Stierwalt, S., Surace, J. A., Thompson, T. A., \& Walter, F. 2015, ApJ, 799, 10 
Blundell, K. M. \& Beasley, A. J. 1998, MNRAS, 299, 165

Bohlin, R. C., Savage, B. D., \& Drake, J. F. 1978, ApJ, 224, 132

Bolatto, A. D., Leroy, A. K., Rosolowsky, E., Walter, F., \& Blitz, L. 2008, ApJ, 686, 948

Bolatto, A. D., Wolfire, M., \& Leroy, A. K. 2013, ARA\&A, 51, 207

Bouché, N., Cresci, G., Davies, R., Eisenhauer, F., Förster Schreiber, N. M., Genzel, R., Gillessen, S., Lehnert, M., Lutz, D., Nesvadba, N., Shapiro, K. L., Sternberg, A., Tacconi, L. J., Verma, A., Cimatti, A., Daddi, E., Renzini, A., Erb, D. K., Shapley, A., \& Steidel, C. C. 2007, ApJ, 671, 303

Cicone, C., Maiolino, R., Sturm, E., Graciá-Carpio, J., Feruglio, C., Neri, R., Aalto, S., Davies, R., Fiore, F., Fischer, J., García-Burillo, S., González-Alfonso, E., Hailey-Dunsheath, S., Piconcelli, E., \& Veilleux, S. 2014, A\&A, 562, A21

Clemens, M. S., Scaife, A., Vega, O., \& Bressan, A. 2010, MNRAS, 405, 887

Clemens, M. S., Vega, O., Bressan, A., Granato, G. L., Silva, L., \& Panuzzo, P. 2008, A\&A, 477, 95

Clements, D. L., McDowell, J. C., Shaked, S., Baker, A. C., Borne, K., Colina, L., Lamb, S. A., \& Mundell, C. 2002, ApJ, 581, 974

Comastri, A. 2004, in Astrophysics and Space Science Library, Vol. 308, Supermassive Black Holes in the Distant Universe, ed. A. J. Barger, 245

Condon, J. J. 1992, ARA\&A, 30, 575

Condon, J. J., Anderson, M. L., \& Helou, G. 1991a, ApJ, 376, 95

Condon, J. J., Cotton, W. D., Greisen, E. W., Yin, Q. F., Perley, R. A., Taylor, G. B., \& Broderick, J. J. 1998, AJ, 115, 1693

Condon, J. J., Helou, G., Sanders, D. B., \& Soifer, B. T. 1990, ApJS, 73, 359 
—. 1996, ApJS, 103, 81

Condon, J. J., Huang, Z.-P., Yin, Q. F., \& Thuan, T. X. 1991b, ApJ, 378, 65

Condon, J. J. \& Yin, Q. F. 1990, ApJ, 357, 97

Daddi, E., Elbaz, D., Walter, F., Bournaud, F., Salmi, F., Carilli, C., Dannerbauer, H., Dickinson, M., Monaco, P., \& Riechers, D. 2010, ApJ, 714, L118

Davis, S. W., Jiang, Y.-F., Stone, J. M., \& Murray, N. 2014, ApJ, 796, 107

Davis, T. A., Bureau, M., Cappellari, M., Sarzi, M., \& Blitz, L. 2013, Nature, 494, 328

Díaz-Santos, T., Armus, L., Charmandaris, V., Stierwalt, S., Murphy, E. J., Haan, S., Inami, H., Malhotra, S., Meijerink, R., Stacey, G., Petric, A. O., Evans, A. S., Veilleux, S., van der Werf, P. P., Lord, S., Lu, N., Howell, J. H., Appleton, P., Mazzarella, J. M., Surace, J. A., Xu, C. K., Schulz, B., Sanders, D. B., Bridge, C., Chan, B. H. P., Frayer, D. T., Iwasawa, K., Melbourne, J., \& Sturm, E. 2013, ApJ, 774,68

Downes, D. \& Eckart, A. 2007, A\&A, 468, L57

Downes, D. \& Solomon, P. M. 1998, ApJ, 507, 615

Draine, B. T. \& Lazarian, A. 1998, ApJ, 508, 157

Dumas, G., Schinnerer, E., Tabatabaei, F. S., Beck, R., Velusamy, T., \& Murphy, E. 2011, AJ, 141, 41

Engel, H., Davies, R. I., Genzel, R., Tacconi, L. J., Sturm, E., \& Downes, D. 2011, ApJ, 729, 58

Evans, A. S., Mazzarella, J. M., Surace, J. A., \& Sanders, D. B. 2002, ApJ, 580, 749

Fukui, Y. \& Kawamura, A. 2010, ARA\&A, 48, 547

Gao, Y. \& Solomon, P. M. 2004a, ApJS, 152, 63 
-. 2004b, ApJ, 606, 271

Genzel, R., Tacconi, L. J., Gracia-Carpio, J., Sternberg, A., Cooper, M. C., Shapiro, K., Bolatto, A., Bouché, N., Bournaud, F., Burkert, A., Combes, F., Comerford, J., Cox, P., Davis, M., Schreiber, N. M. F., Garcia-Burillo, S., Lutz, D., Naab, T., Neri, R., Omont, A., Shapley, A., \& Weiner, B. 2010, MNRAS, 407, 2091

Genzel, R., Tacconi, L. J., Rigopoulou, D., Lutz, D., \& Tecza, M. 2001, ApJ, 563, 527

González-Alfonso, E., Fischer, J., Graciá-Carpio, J., Sturm, E., Hailey-Dunsheath, S., Lutz, D., Poglitsch, A., Contursi, A., Feuchtgruber, H., Veilleux, S., Spoon, H. W. W., Verma, A., Christopher, N., Davies, R., Sternberg, A., Genzel, R., \& Tacconi, L. 2012, A\&A, 541, A4

Greve, T. R., Papadopoulos, P. P., Gao, Y., \& Radford, S. J. E. 2009, ApJ, 692, 1432

Haan, S., Surace, J. A., Armus, L., Evans, A. S., Howell, J. H., Mazzarella, J. M., Kim, D. C., Vavilkin, T., Inami, H., Sanders, D. B., Petric, A., Bridge, C. R., Melbourne, J. L., Charmandaris, V., Diaz-Santos, T., Murphy, E. J., U, V., Stierwalt, S., \& Marshall, J. A. 2011, AJ, 141, 100

Haas, M., Klaas, U., Müller, S. A. H., Chini, R., \& Coulson, I. 2001, A\&A, 367, L9

Herrero-Illana, R., Pérez-Torres, M. Á., \& Alberdi, A. 2012, A\&A, 540, L5

Hopkins, P. F., Murray, N., Quataert, E., \& Thompson, T. A. 2010, MNRAS, 401, L19

Ilovaisky, S. A. \& Lequeux, J. 1972, A\&A, 20, 347

Imanishi, M., Nakanishi, K., Tamura, Y., Oi, N., \& Kohno, K. 2007, AJ, 134, 2366

Imanishi, M. \& Saito, Y. 2014, ApJ, 780, 106

Iono, D., Wilson, C. D., Yun, M. S., Baker, A. J., Petitpas, G. R., Peck, A. B., Krips, M., Cox, T. J., Matsushita, S., Mihos, J. C., \& Pihlstrom, Y. 2009, ApJ, 695, 1537 
Iwasawa, K., Sanders, D. B., Evans, A. S., Mazzarella, J. M., Armus, L., \& Surace, J. A. 2009, ApJ, 695, L103

Iwasawa, K., Sanders, D. B., Evans, A. S., Trentham, N., Miniutti, G., \& Spoon, H. W. W. 2005, MNRAS, 357, 565

Iwasawa, K., Sanders, D. B., Teng, S. H., U, V., Armus, L., Evans, A. S., Howell, J. H., Komossa, S., Mazzarella, J. M., Petric, A. O., Surace, J. A., Vavilkin, T., Veilleux, S., \& Trentham, N. 2011, A\&A, 529, A106

Johnson, K. E., Leroy, A. K., Indebetouw, R., Brogan, C. L., Whitmore, B. C., Hibbard, J., Sheth, K., \& Evans, A. S. 2015, ApJ, 806, 35

Kellermann, K. I., Sramek, R., Schmidt, M., Shaffer, D. B., \& Green, R. 1989, AJ, 98,1195

Kennicutt, Jr., R. C. 1998, ApJ, 498, 541

Keto, E., Ho, L. C., \& Lo, K.-Y. 2005, ApJ, 635, 1062

Kim, D.-C. \& Sanders, D. B. 1998, ApJS, 119, 41

Krumholz, M. R., Dekel, A., \& McKee, C. F. 2012, ApJ, 745, 69

Krumholz, M. R. \& McKee, C. F. 2005a, ApJ, 630, 250

-. 2005b, ApJ, 630, 250

Krumholz, M. R. \& Thompson, T. A. 2013, MNRAS, 434, 2329

Le Floc'h, E., Papovich, C., Dole, H., Bell, E. F., Lagache, G., Rieke, G. H., Egami, E., Pérez-González, P. G., Alonso-Herrero, A., Rieke, M. J., Blaylock, M., Engelbracht, C. W., Gordon, K. D., Hines, D. C., Misselt, K. A., Morrison, J. E., \& Mould, J. 2005, ApJ, 632, 169

Leroy, A. K., Bigiel, F., de Blok, W. J. G., Boissier, S., Bolatto, A., Brinks, E., Madore, B., Munoz-Mateos, J.-C., Murphy, E., Sandstrom, K., Schruba, A., \& Walter, F. 2012, AJ, 144, 3 
Leroy, A. K., Evans, A. S., Momjian, E., Murphy, E., Ott, J., Armus, L., Condon, J., Haan, S., Mazzarella, J. M., Meier, D. S., Privon, G. C., Schinnerer, E., Surace, J., \& Walter, F. 2011, ApJ, 739, L25

Leroy, A. K., Walter, F., Martini, P., Roussel, H., Sandstrom, K., Ott, J., Weiss, A., Bolatto, A. D., Schuster, K., \& Dessauges-Zavadsky, M. 2015, ApJ, 814, 83

Leroy, A. K., Walter, F., Sandstrom, K., Schruba, A., Munoz-Mateos, J.-C., Bigiel, F., Bolatto, A., Brinks, E., de Blok, W. J. G., Meidt, S., Rix, H.-W., Rosolowsky, E., Schinnerer, E., Schuster, K.-F., \& Usero, A. 2013, AJ, 146, 19

Li, A. \& Draine, B. T. 2001, ApJ, 554, 778

Liu, L., Gao, Y., \& Greve, T. R. 2015, ApJ, 805, 31

Lonsdale, C. J., Diamond, P. J., Thrall, H., Smith, H. E., \& Lonsdale, C. J. 2006a, ApJ, 647, 185

Lonsdale, C. J., Farrah, D., \& Smith, H. E. Ultraluminous Infrared Galaxies, ed. J. W. Mason, 285

Lonsdale, C. J., Lonsdale, C. J., Smith, H. E., \& Diamond, P. J. 2003, ApJ, 592, 804

Lutz, D., Berta, S., Contursi, A., Förster Schreiber, N. M., Genzel, R., Graciá-Carpio, J., Herrera-Camus, R., Netzer, H., Sturm, E., Tacconi, L. J., Tadaki, K., \& Veilleux, S. 2016, A\&A, 591, A136

Lutz, D., Genzel, R., Sternberg, A., Netzer, H., Kunze, D., Rigopoulou, D., Sturm, E., Egami, E., Feuchtgruber, H., Moorwood, A. F. M., \& de Graauw, T. 1996, A\&A, 315, L137

Magnelli, B., Elbaz, D., Chary, R. R., Dickinson, M., Le Borgne, D., Frayer, D. T., \& Willmer, C. N. A. 2011, A\&A, 528, A35

Malhotra, S., Kaufman, M. J., Hollenbach, D., Helou, G., Rubin, R. H., Brauher, J., Dale, D., Lu, N. Y., Lord, S., Stacey, G., Contursi, A., Hunter, D. A., \& Dinerstein, H. 2001, ApJ, 561, 766 
Marvil, J., Owen, F., \& Eilek, J. 2015, AJ, 149, 32

Mazzarella, J. M., Soifer, B. T., Graham, J. R., Hafer, C. I., Neugebauer, G., \& Matthews, K. 1992, AJ, 103, 413

McBride, J., Quataert, E., Heiles, C., \& Bauermeister, A. 2014, ApJ, 780, 182

McMullin, J. P., Waters, B., Schiebel, D., Young, W., \& Golap, K. 2007, in Astronomical Society of the Pacific Conference Series, Vol. 376, Astronomical Data Analysis Software and Systems XVI, ed. R. A. Shaw, F. Hill, \& D. J. Bell, 127

Meier, D. S., Walter, F., Bolatto, A. D., Leroy, A. K., Ott, J., Rosolowsky, E., Veilleux, S., Warren, S. R., Weiß, A., Zwaan, M. A., \& Zschaechner, L. K. 2015, ApJ, 801, 63

Meurer, G. R., Heckman, T. M., Lehnert, M. D., Leitherer, C., \& Lowenthal, J. 1997, AJ, 114,54

Miettinen, O. 2014, A\&A, 562, A3

Mundell, C. G., Ferruit, P., \& Pedlar, A. 2001, ApJ, 560, 168

Murphy, E. J. 2009, ApJ, 706, 482

-. 2013, ApJ, 777, 58

Murphy, E. J., Bremseth, J., Mason, B. S., Condon, J. J., Schinnerer, E., Aniano, G., Armus, L., Helou, G., Turner, J. L., \& Jarrett, T. H. 2012, ApJ, 761, 97

Murphy, E. J., Helou, G., Braun, R., Kenney, J. D. P., Armus, L., Calzetti, D., Draine, B. T., Kennicutt, Jr., R. C., Roussel, H., Walter, F., Bendo, G. J., Buckalew, B., Dale, D. A., Engelbracht, C. W., Smith, J. D. T., \& Thornley, M. D. 2006, ApJ, 651, L111

Murphy, E. J., Helou, G., Condon, J. J., Schinnerer, E., Turner, J. L., Beck, R., Mason, B. S., Chary, R.-R., \& Armus, L. 2010, ApJ, 709, L108 
Murphy, E. J., Stierwalt, S., Armus, L., Condon, J. J., \& Evans, A. S. 2013, ApJ, 768, 2

Murray, N., Quataert, E., \& Thompson, T. A. 2005, ApJ, 618, 569

Narayanan, D., Krumholz, M. R., Ostriker, E. C., \& Hernquist, L. 2012, MNRAS, 421,3127

Neff, S. G., Ulvestad, J. S., \& Teng, S. H. 2004, ApJ, 611, 186

Norris, R. P. 1988, MNRAS, 230, 345

Ostriker, E. C. \& Shetty, R. 2011, ApJ, 731, 41

Papadopoulos, P. P., van der Werf, P., Xilouris, E., Isaak, K. G., \& Gao, Y. 2012, ApJ, 751, 10

Parra, R., Conway, J. E., Diamond, P. J., Thrall, H., Lonsdale, C. J., Lonsdale, C. J., \& Smith, H. E. 2007, ApJ, 659, 314

Petric, A. O., Armus, L., Howell, J., Chan, B., Mazzarella, J. M., Evans, A. S., Surace, J. A., Sanders, D., Appleton, P., Charmandaris, V., Díaz-Santos, T., Frayer, D., Haan, S., Inami, H., Iwasawa, K., Kim, D., Madore, B., Marshall, J., Spoon, H., Stierwalt, S., Sturm, E., U, V., Vavilkin, T., \& Veilleux, S. 2011, ApJ, 730, 28

Pooley, G. G. 1969, MNRAS, 144, 101

Rabidoux, K., Pisano, D. J., Kepley, A. A., Johnson, K. E., \& Balser, D. S. 2014, ApJ, 780, 19

Rangwala, N., Maloney, P. R., Glenn, J., Wilson, C. D., Rykala, A., Isaak, K., Baes, M., Bendo, G. J., Boselli, A., Bradford, C. M., Clements, D. L., Cooray, A., Fulton, T., Imhof, P., Kamenetzky, J., Madden, S. C., Mentuch, E., Sacchi, N., Sauvage, M., Schirm, M. R. P., Smith, M. W. L., Spinoglio, L., \& Wolfire, M. 2011, ApJ, 743,94 
Rangwala, N., Maloney, P. R., Wilson, C. D., Glenn, J., Kamenetzky, J., \& Spinoglio, L. 2015, ApJ, 806, 17

Robishaw, T., Quataert, E., \& Heiles, C. 2008, ApJ, 680, 981

Rodríguez-Rico, C. A., Goss, W. M., Viallefond, F., Zhao, J.-H., Gómez, Y., \& Anantharamaiah, K. R. 2005, ApJ, 633, 198

Rovilos, E., Diamond, P. J., Lonsdale, C. J., Lonsdale, C. J., \& Smith, H. E. 2003, MNRAS, 342, 373

Rupke, D. S. N., Veilleux, S., \& Baker, A. J. 2008, ApJ, 674, 172

Sakamoto, K., Aalto, S., Wilner, D. J., Black, J. H., Conway, J. E., Costagliola, F., Peck, A. B., Spaans, M., Wang, J., \& Wiedner, M. C. 2009, ApJ, 700, L104

Sakamoto, K., Scoville, N. Z., Yun, M. S., Crosas, M., Genzel, R., \& Tacconi, L. J. 1999, ApJ, 514, 68

Sakamoto, K., Wang, J., Wiedner, M. C., Wang, Z., Peck, A. B., Zhang, Q., Petitpas, G. R., Ho, P. T. P., \& Wilner, D. J. 2008, ApJ, 684, 957

Sanders, D. B., Mazzarella, J. M., Kim, D.-C., Surace, J. A., \& Soifer, B. T. 2003, AJ, 126, 1607

Sanders, D. B. \& Mirabel, I. F. 1996, ARA\&A, 34, 749

Sanders, D. B., Scoville, N. Z., \& Soifer, B. T. 1988a, ApJ, 335, L1

Sanders, D. B., Soifer, B. T., Elias, J. H., Madore, B. F., Matthews, K., Neugebauer, G., \& Scoville, N. Z. 1988b, ApJ, 325, 74

Sault, R. J. \& Wieringa, M. H. 1994, A\&AS, 108

Scoville, N. 2003, Journal of Korean Astronomical Society, 36, 167

Scoville, N., Aussel, H., Sheth, K., Scott, K. S., Sanders, D., Ivison, R., Pope, A., Capak, P., Vanden Bout, P., Manohar, S., Kartaltepe, J., Robertson, B., \& Lilly, S. 2014, ApJ, 783, 84 
Scoville, N., Murchikova, L., Walter, F., Vlahakis, C., Koda, J., Vanden Bout, P., Barnes, J., Hernquist, L., Sheth, K., Yun, M., Sanders, D., Armus, L., Cox, P., Thompson, T., Robertson, B., Zschaechner, L., Tacconi, L., Torrey, P., Hayward, C. C., Genzel, R., Hopkins, P., van der Werf, P., \& Decarli, R. 2016, ArXiv e-prints

Scoville, N., Sheth, K., Walter, F., Manohar, S., Zschaechner, L., Yun, M., Koda, J., Sanders, D., Murchikova, L., Thompson, T., Robertson, B., Genzel, R., Hernquist, L., Tacconi, L., Brown, R., Narayanan, D., Hayward, C. C., Barnes, J., Kartaltepe, J., Davies, R., van der Werf, P., \& Fomalont, E. 2015, ApJ, 800, 70

Scoville, N. Z., Evans, A. S., Dinshaw, N., Thompson, R., Rieke, M., Schneider, G., Low, F. J., Hines, D., Stobie, B., Becklin, E., \& Epps, H. 1998, ApJ, 492, L107

Semenov, D., Henning, T., Helling, C., Ilgner, M., \& Sedlmayr, E. 2003, A\&A, 410, 611

Semenov, V. A., Kravtsov, A. V., \& Gnedin, N. Y. 2015, ArXiv e-prints

Shetty, R., Glover, S. C., Dullemond, C. P., \& Klessen, R. S. 2011, MNRAS, 412, 1686

Smith, H. E., Lonsdale, C. J., Lonsdale, C. J., \& Diamond, P. J. 1998, ApJ, 493, L17

Socrates, A., Davis, S. W., \& Ramirez-Ruiz, E. 2008, ApJ, 687, 202

Socrates, A. \& Sironi, L. 2013, ApJ, 772, L21

Soifer, B. T., Neugebauer, G., Matthews, K., Becklin, E. E., Ressler, M., Werner, M. W., Weinberger, A. J., \& Egami, E. 1999, ApJ, 513, 207

Soifer, B. T., Neugebauer, G., Matthews, K., Egami, E., Becklin, E. E., Weinberger, A. J., Ressler, M., Werner, M. W., Evans, A. S., Scoville, N. Z., Surace, J. A., \& Condon, J. J. 2000, AJ, 119, 509

Solomon, P. M., Downes, D., Radford, S. J. E., \& Barrett, J. W. 1997, ApJ, 478, 144 
Spoon, H. W. W., Marshall, J. A., Houck, J. R., Elitzur, M., Hao, L., Armus, L., Brandl, B. R., \& Charmandaris, V. 2007, ApJ, 654, L49

Stierwalt, S., Armus, L., Surace, J. A., Inami, H., Petric, A. O., Diaz-Santos, T., Haan, S., Charmandaris, V., Howell, J., Kim, D. C., Marshall, J., Mazzarella, J. M., Spoon, H. W. W., Veilleux, S., Evans, A., Sanders, D. B., Appleton, P., Bothun, G., Bridge, C. R., Chan, B., Frayer, D., Iwasawa, K., Kewley, L. J., Lord, S., Madore, B. F., Melbourne, J. E., Murphy, E. J., Rich, J. A., Schulz, B., Sturm, E., Vavilkin, T., \& Xu, K. 2013, ApJS, 206, 1

Tabatabaei, F. S., Schinnerer, E., Murphy, E. J., Beck, R., Groves, B., Meidt, S., Krause, M., Rix, H.-W., Sandstrom, K., Crocker, A. F., Galametz, M., Helou, G., Wilson, C. D., Kennicutt, R., Calzetti, D., Draine, B., Aniano, G., Dale, D., Dumas, G., Engelbracht, C. W., Gordon, K. D., Hinz, J., Kreckel, K., Montiel, E., \& Roussel, H. 2013, A\&A, 552, A19

Tacconi, L. J., Genzel, R., Smail, I., Neri, R., Chapman, S. C., Ivison, R. J., Blain, A., Cox, P., Omont, A., Bertoldi, F., Greve, T., Förster Schreiber, N. M., Genel, S., Lutz, D., Swinbank, A. M., Shapley, A. E., Erb, D. K., Cimatti, A., Daddi, E., \& Baker, A. J. 2008, ApJ, 680, 246

Tan, J. C. 2000, ApJ, 536, 173

Tan, Q., Gao, Y., \& Xia, X. Y. 2009, in Astronomical Society of the Pacific Conference Series, Vol. 408, The Starburst-AGN Connection, ed. W. Wang, Z. Yang, Z. Luo, \& Z. Chen, 179

Thompson, T. A., Quataert, E., \& Murray, N. 2005, ApJ, 630, 167

Tunnard, R., Greve, T. R., Garcia-Burillo, S., Graciá Carpio, J., Fischer, J., Fuente, A., González-Alfonso, E., Hailey-Dunsheath, S., Neri, R., Sturm, E., Usero, A., \& Planesas, P. 2015, ApJ, 800, 25

Ulvestad, J. S., Wrobel, J. M., \& Carilli, C. L. 1999, ApJ, 516, 127 
Varenius, E., Conway, J. E., Martí-Vidal, I., Aalto, S., Barcos-Muñoz, L., König, S., Pérez-Torres, M. A., Deller, A. T., Moldón, J., Gallagher, J. S., Yoast-Hull, T. M., Horellou, C., Morabito, L. K., Alberdi, A., Jackson, N., Beswick, R., Carozzi, T. D., Wucknitz, O., \& Ramírez-Olivencia, N. 2016, ArXiv e-prints

Veilleux, S., Cecil, G., \& Bland-Hawthorn, J. 2005, ARA\&A, 43, 769

Veilleux, S., Kim, D.-C., Sanders, D. B., Mazzarella, J. M., \& Soifer, B. T. 1995, ApJS, 98, 171

Veilleux, S., Sanders, D. B., \& Kim, D.-C. 1997, ApJ, 484, 92

Wei, L. H., Keto, E., \& Ho, L. C. 2012, ApJ, 750, 136

Williams, P. K. G. \& Bower, G. C. 2010, ApJ, 710, 1462

Wilson, C. D., Rangwala, N., Glenn, J., Maloney, P. R., Spinoglio, L., \& PereiraSantaella, M. 2014, ApJ, 789, L36

Yun, M. S., Reddy, N. A., \& Condon, J. J. 2001, ApJ, 554, 803

Yun, M. S., Reddy, N. A., Scoville, N. Z., Frayer, D. T., Robson, E. I., \& Tilanus, R. P. J. 2004, ApJ, 601, 723 